\title{
FLOW AND MORPHOLOGICAL CONDITIONS ASSOCIATED WITH THE DIRECTIONAL SOLIDIFICATION OF AQUEOUS AMMONIUM CHLORIDE
}

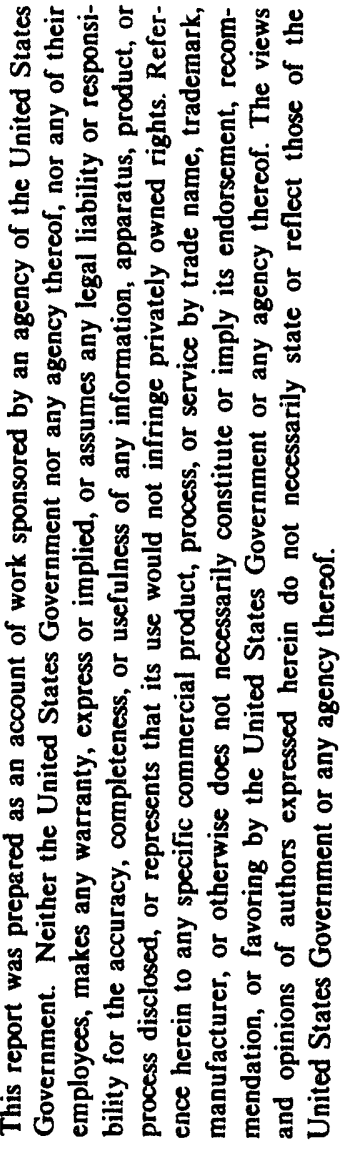

\author{
by \\ C.S. Magirl and F.P. Incropera \\ Heat Transfer Laboratory \\ School of Mechanical Engineering \\ Purdue University \\ West Lafayette, Indiana 47907 \\ Annual Performance Report Number \\ DOE/ER/13759-6 \\ DE93 008886
}

DOE/ER/13759--6

\author{
Submitted to \\ Department of Energy \\ Office of Energy Research \\ In Partial Fulfillment of Requirements for \\ Award No. DE-FG02-87ER 13759
}

on

Effect of Externally Induced Forces

on Solidification of Binary Mixtures

January 1993 MASTER 


\section{TABLE OF CONTENTS}

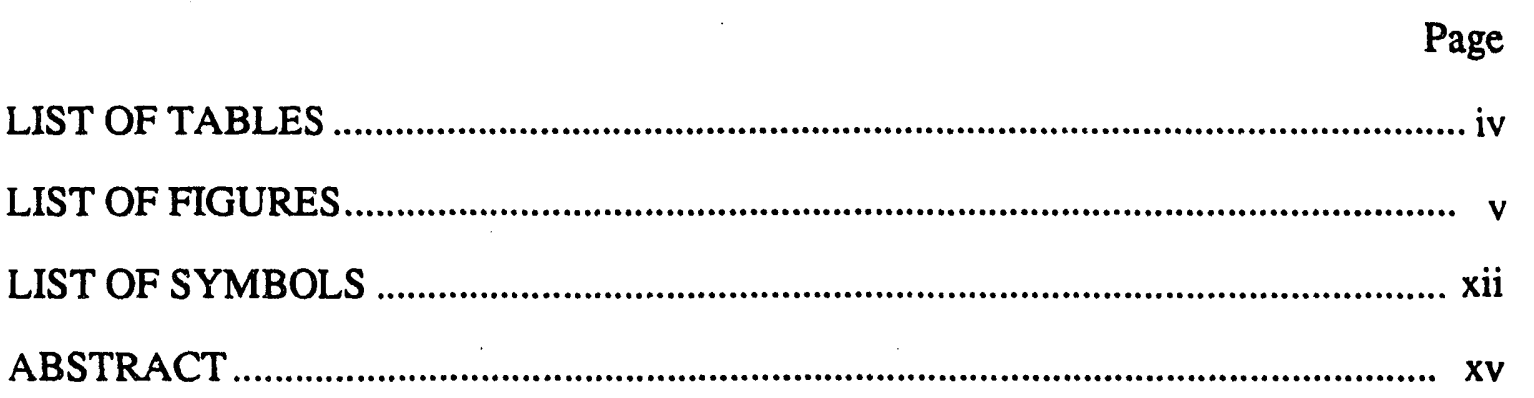

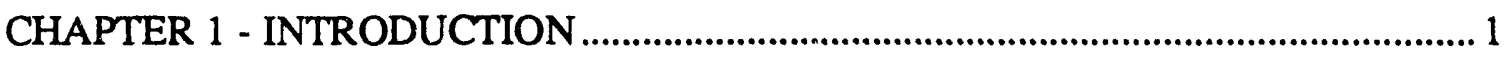

1.1 Solidification of Multicomponent Mixtures...................................................... 1

1.2 The Macroscopic Physics of Soliditication of a Binary Alloy................................ 3

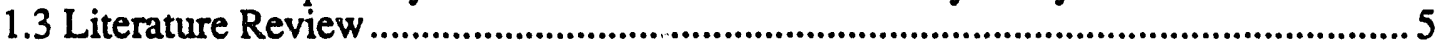

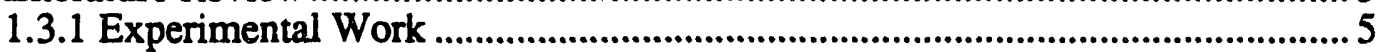

1.3.2 Analytical and Numerical Investigations ..................................................

1.3.3 Macrosegregation Control Schemes................................................................. 9

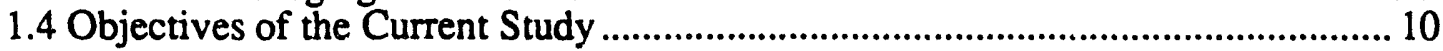

CHAPTER 2 - EXPERIMENTAL PROCEDURES ................................................. 12

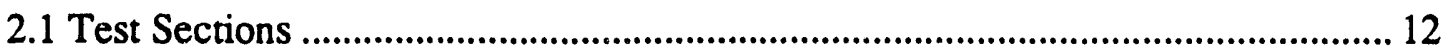

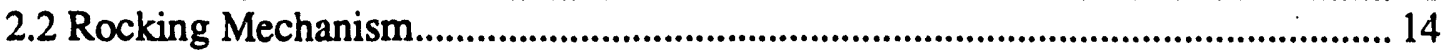

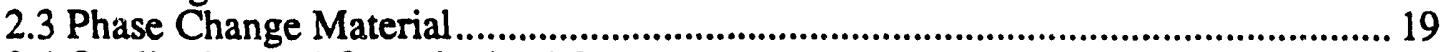

2.4 Qualitative and Quantitative Measurements ................................................... 21

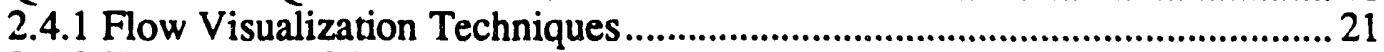

2.4.2 Temperature Measurement.................................................................... 22

2.4.3 Species Concentration Measurement ................................................... 24

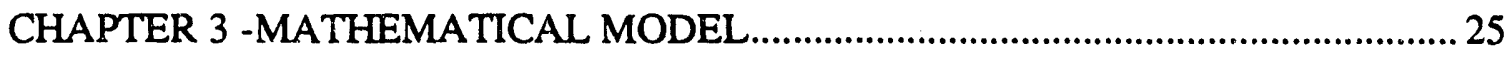

3.1 Static Continuum Model .................................................................................... 25

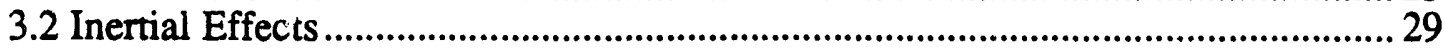

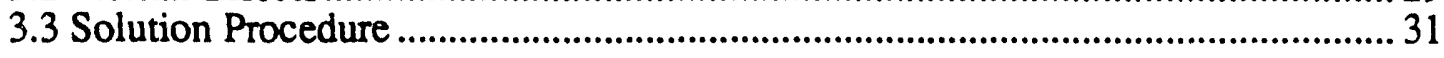

CHAPTER 4 - EXPERIMENTAL RESULTS OF STATIC SOLIDIFICATION ........... 32

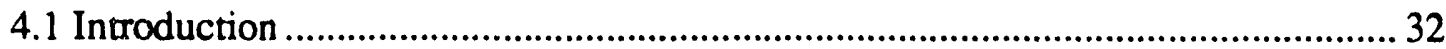

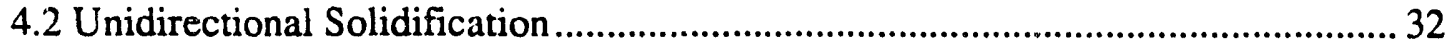

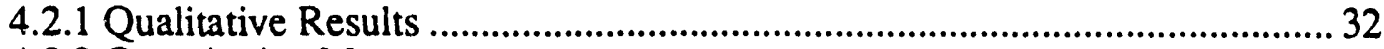

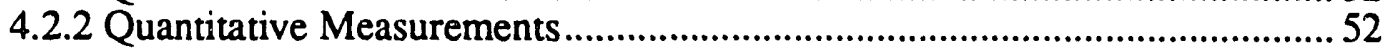


4.3 Side and Bottom Wall Chilling .................................................................................. 54

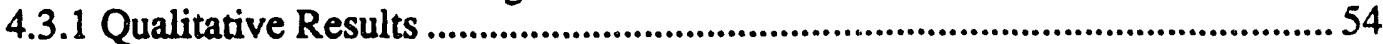

4.3.2 Quantitative Measurements......................................................................... 65

CHAPTER 5 - EXPERIMENTAL RESULTS OF SOLIDIFICATION DURING

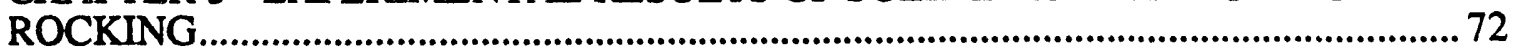

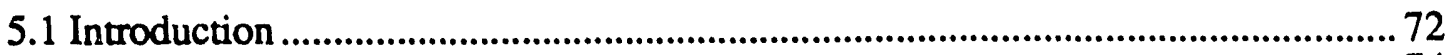

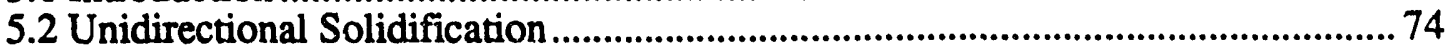

5.2.1 Qualitative Observations ..................................................................................

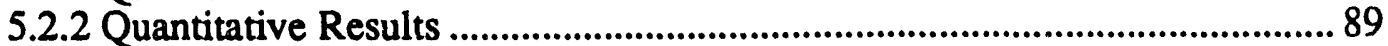

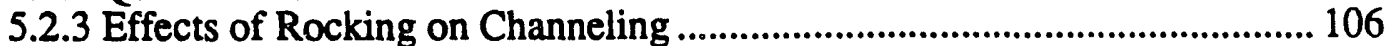

5.3 Side and Bottom Wall Chilling ................................................................................ 110

5.3.1 Qualitative Observations ..................................................................................... 110

5.3.2 Quantitative Results ................................................................................... 121

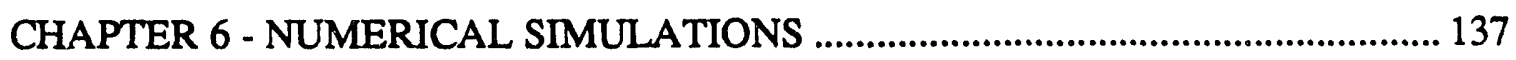

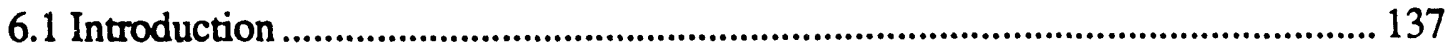

6.2 Static Unidirectional Solidification.................................................................... 141

6.3 Unidirectional Solidification During Rocking ................................................... 153

6.4 Side and Bottom Wall Chilling ................................................................................ 178

CHAPTER 7 - CONCLUSIONS AND RECOMMENDATIONS .................................... 190

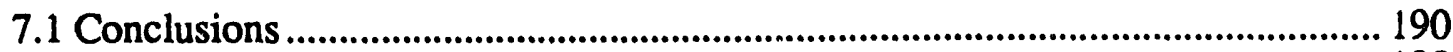

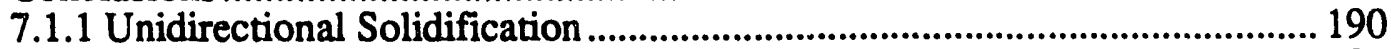

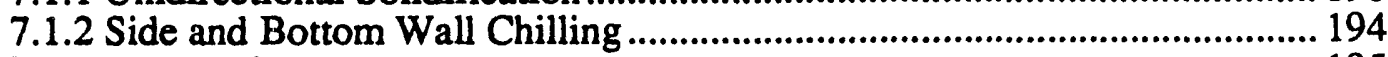

7.2 Recommendations .................................................................................................. 195

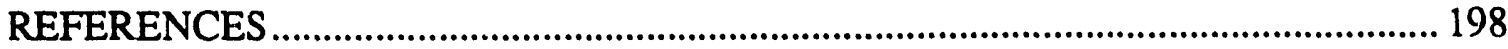

\section{APPENDICES}

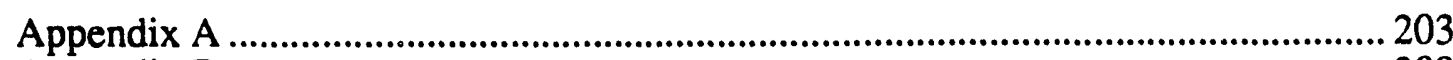

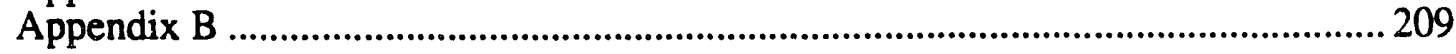




\section{LIST OF TABLES}

Table

Page

5.1 Experimental conditions for static and rocking experiments .................................. 73

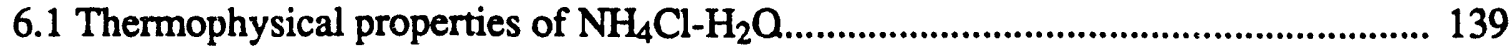




\section{LIST OF FIGURES}

Figure

Page

2.1 Schematic of shadowgraph visualization optics and test section........................ 13

2.2 Design of four-bar linkage showing (a) dimensions of each bar and

(b) predicted and actual linkage performance.

2.3 Complete table-top experimental apparatus................................................. 17

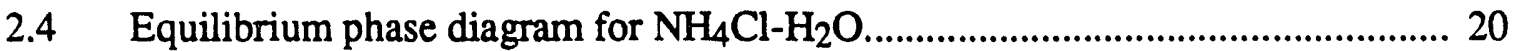

2.5 Locations of temperature and species concentration sensors for

a) test section I, and b) test section II.

4.1 Top view of nascent crystal growth on bottom heat exchanger surface:

(a) single crystal grown very slowly in order to show denciritic structure

(b) 5 , (c) 10 , and (d) $15 \mathrm{~s}$ after the incipience of crystal nucleation when

$-30^{\circ} \mathrm{C}$ coolant was suddenly applied to the heat exchanger.

4.2 Incipient salt fingering at one minute using (a) shadowgraph, and

(b) fluorescein dye illuminated with a sheet of argon-ion laser light.

4.3 (a) Time exposure of slurry zone above advancing liquidus front during unidirectional solidification (UDS) at 300 s showing discrete fountain sites where crystals are ejected into the melt. (b) Overhead view of equiaxed mounds resulting from the slurry.

4.4 Shadowgraph of early development of salt fingers and transformation into organized plumes emanating from channels: (a) 1.5 , (b) 3 , (c) 5 , (d) 7.5 . (e) 10 , (f) 15, (g) 20, and (h) $25 \mathrm{~min}$.

4.5 Shadowgraph images of UDS system in first hour showing the advancing liquidus front and development of plumes emanating from channels in the mushy region: (a) 15 , (b) 30 , (c) 45 , and (d) $60 \mathrm{~min}$.

4.6 Shadowgraph images of UDS system during the second hour showing organization and strengthening of plumes, suppression of salt fingering, and double-diffusive convection within the melt: (a) 75, (b) 90 , (c) 105 , and (d) $120 \mathrm{~min}$. 
Figure

4.7 UDS shadowgraph image at 106 minutes of three helical plumes showing double-diffusive layering and cold 'wisps' of fluid falling from bends in the plume. Waves in the plume propagate upwards.

4.8 Use of fluorescein dye to illustrate double-diffusive convection associated with ascending plumes in a UDS process: (a) pair of plumes on the left-hand side of the test section with associated double-diffusive layering and thermally driven wisps, and (b) single plume on the right-hand side of the test section showing the thermally driven cells.

4.9 Schematic showing flow patterns adjacent to an oscillating plume.

4.10 Particle tracking with time exposure of 3 seconds showing cellular flows around an ascending, meandering plume.

4.11 Shadowgraph of double-diffusive layers in the melt of a UDS system at approximately 2 hours.

4.12 Crystalline grain structure of mushy region and channels from (a) top, and (b) side.

4.13 Temperature histories at selected heights for unidirectional solidification under static conditions.

4.14 Liquid composition of ammonium chloride during unidirectional solidification under static conditions.

4.15 Shadowgraph of early solidification for concurrent side and bottom wall chilling: (a) 1 , (b) 2.5 , (c) 5 , and (d) $7.5 \mathrm{~min}$.

4.16 Shadowgraph showing double-diffusive cell development, rapid growth of the liquidus front, and crystal ejection in the form of slurries: (a) 10 ,

(b) 12.5 , (c) 15 , and (d) $20 \mathrm{~min}$.

4.17 Localized view of slurry zone at $12.5 \mathrm{~min}$ : (a) shadowgraph and (b) time exposure of $20 \mathrm{~s}$

4.18 Shadowgraph of late development showing advancing liquidus front, maturing convection cells, and impedance of ascending plumes by the cells: (a) 30, (b) 40, (c) 50, and (d) 60 min. Note: upper convection cell is free of a mushy region.

4.19 Conditions within a series of double diffusive convection cells at 48 minutes: (a) shadowgraph, (b) particle tracking, and (c) schematic.

4.20 Fluorescein dye injection into side wall mushy region at 45 minutes showing double-diffusive cells in the melt as well as a plunie emanating from an A-segregate type channel. 
4.21 Measured cooling curves along vertical thermocouple array located at $\mathrm{x}=38.1 \mathrm{~mm}$ under side and bottom chilling conditions.

4.22 Measured cooling curves under side and bottom chilling conditions at

(a) $y=76.2 \mathrm{~mm}$, and (b) $\mathrm{y}=50.8 \mathrm{~mm}$.

4.23 Liquid composition measurements at $x=25.4 \mathrm{~mm}$ (open symbols)

and at the centerline (closed symbols).

5.1 Shadowgraph images of early solidification for case UDS 1R: (a) $1\left(\theta=22^{\circ}\right)$,

(b) $2\left(\theta=36^{\circ}\right)$, (c) $5\left(\theta=38^{\circ}\right)$, and (d) $7 \min \left(\theta=38^{\circ}\right)$ 75

5.2 Three-dimensional flow characteristics of the rocking test section

5.3 Shadowgraph images for case UDS $1 \mathrm{R}$ showing robust salt fingering and mixing: (a) $15\left(\theta=-4^{\circ}\right)$, (b) $20\left(\theta=38^{\circ}\right)$, and (c) $30 \min \left(\theta=0^{\circ}\right)$.

5.4 Shadowgraph images at $45 \mathrm{~min}$ for case UDS 1R showing advective sweeping of the liquidus interface as the test section rotates from (a) $+13^{\circ}$ to (b) $-38^{\circ}$.

5.5 Shadowgraph images of later stages of solidification for experiment:

UDS $1 R$ (a) $60\left(\theta=0^{\circ}\right)$, (b) $75\left(\theta=0^{\circ}\right)$, (c) $92 \min \left(\theta=-3^{\circ}\right)$.

5.6 Sequence of images over $7.5 \mathrm{~s}$ interval $105 \mathrm{~min}$ for case UDS 1R: test section rocks from (a) the vertical position, to (b) $18^{\circ}$, to (c) $40^{\circ}$.

5.7 Final shadowgraph image for case UDS $1 \mathrm{R}$ at $120 \mathrm{~min}\left(\theta=-3^{\circ}\right) \ldots \ldots \ldots \ldots \ldots \ldots . . . . . . . . . . .88$

5.8 Mushy region morphology of case UDS $1 \mathrm{R}$ as viewed from the (a) side and the (b) top. Also top view (c) of statically grown mushy region.

5.9 Temperature histories from experiment UDS 1R. ........................................ 92

5.10 Temperature histories in the melt from $\mathrm{t}=15$ to $19 \mathrm{~min}$..................................93

5.11 Comparison of melt temperatures between static (UDS 1) and rocking (UDS 1R) experiments.

5.12 Temperature histories from experiment UDS 2R. ........................................97

5.13 Temperature histories from experiment UDS 3R. .........................................99

5.14 Temperature histories from experiment UDS 4R. .................................... 100

5.15 Averaged melt temperature histories of upper four thermocouple locations for both rocking and static UDS experiments 
5.16 Standard deviations of temperature fluctuations in the melt for unidirectional solidification from 3600 to $4350 \mathrm{~s}$.

5.17 Liquid composition of ammonium chloride for experiments (a) UDS $1 R$ and (b) UDS 4R.

5.18 Liquid composition of ammonium chloride for experiments (a) UDS $2 R$ and (b) UDS 3R.

5.19 Comparison of liquid composition trends for both rocking and static UDS experiments

5.20 Shadowgraph images of early solidification for case MDS 1R: (a) $1\left(\theta=2^{\circ}\right)$,

(b) $1.5\left(\theta=30^{\circ}\right)$, (c) $3\left(\theta=1^{\circ}\right)$, and (d) $4 \min \left(\theta=-4^{\circ}\right)$.

5.21 Shadowgraph images for case MDS 1R: (a) $5\left(\theta=0^{\circ}\right)$, (b) $6.5\left(\theta=37^{\circ}\right)$,

(c) $9\left(\theta=27^{\circ}\right)$, and (d) $11 \mathrm{~min}\left(\theta=38^{\circ}\right)$

5.22 Shadowgraph images for case MDS 1R: (a) $15\left(\theta=30^{\circ}\right)$, (b) $20\left(\theta=-31^{\circ}\right)$,

(c) $31\left(\theta=37^{\circ}\right)$, (d) $44 \min \left(\theta=4^{\circ}\right)$

5.23 Morphological conditions of experiment MDS $1 \mathrm{R}$ after one hour:

(a) shadowgraph and (b) photograph.

5.24 Schematic showing differences in growth morphology under (a) static and

(b) rocking conditions.

5.25 Temperature histories along vertical thermocouple array located at $\mathrm{x}=38.1 \mathrm{~mm}$ for experiment MDS $1 \mathrm{R}$.

5.26 Temperature histories for experiment MDS $1 \mathrm{R}$ at (a) $\mathrm{y}=76.2 \mathrm{~mm}$ and

(b) $\mathrm{y}=50.8 \mathrm{~mm}$.

5.27 Comparison of melt temperatures for static and rocking experiments (MDS 1 and MDS IR).

5.28 Temperature histories along vertical thermocouple array located at $\mathrm{x}=38.1 \mathrm{~mm}$ for experiment MDS $2 \mathrm{R}$.

5.29 Temperature histories for experiment MDS $2 \mathrm{R}$ at (a) $\mathrm{y}=76.2 \mathrm{~mm}$ and

(b) $\mathrm{y}=50.8 \mathrm{~mm}$.

5.30 Temperature histories along vertical thermocouple array located at $\mathrm{x}=38.1 \mathrm{~mm}$ for experiment MDS $3 \mathrm{R}$.

5.31 Temperature histories for experiment MDS $3 R$ at (a) $y=76.2 \mathrm{~mm}$ and

(b) $\mathrm{y}=50.8 \mathrm{~mm}$ 
Figure

5.32 Standard deviations of temperature fluctuations in the melt for multidirectional solidification from 1900 to $2350 \mathrm{~s}$ 134

5.33 Liquid composition of ammonium chloride at $x=25.4 \mathrm{~mm}$ (open symbols) and at the centerline (closed symbols) for experiments (a) MDS $1 \mathrm{R}$,

(b) MDS 2R, and (c) MDS 3R. 136

6.1 Computational grid of (a) test section I and (b) test section II. 140

6.2 Predicted solidification behavior of case NUDS 1 at $300 \mathrm{~s}$ : (a) velocity vectors, (b) streamlines, (c) isotherms, and (d) liquid isocomposition. 142

6.3 Predicted solidification behavior of case NUDS 1 at $600 \mathrm{~s}$ : (a) velocity vectors, (b) streamlines, (c) isotherms, and (d) liquid isocomposition.

6.4 Predicted solidification behavior of case NUDS 1 at $900 \mathrm{~s}$ : (a) velocity vectors,

(b) streamlines, (c) isotherms, and (d) liquid isocomposition.

6.5 Predicted solidification behavior of case NUDS 1 at $1800 \mathrm{~s}$ : (a) velocity vectors,

(b) streamlines, (c) isotherms, and (d) liquid isocomposition

6.6 Predicted solidification behavior of case NUDS 1 at $3600 \mathrm{~s}$ : (a) velocity vectors,

(b) streamlines, (c) isotherms, and (d) liquid isocomposition.

6.7 Species macrosegregation of case NUDS 1 at (a) $1800 \mathrm{~s}$ and (b) $3600 \mathrm{~s}$. 150

6.8 Numerical predictions of temperature histories for case UDS 1 in (a) the melt and (b) the mushy region.

6.9 Predicted solidification behavior of case NUDS $4 \mathrm{R}$ at $300 \mathrm{~s}$ : (a) velocity vectors,

(b) streamlines, (c) isotherms, and (d) liquid isocomposition. 155

6.10 Predicted solidification behavior of case NUDS $4 \mathrm{R}$ at $600 \mathrm{~s}$ : (a) velocity vectors, (b) streamlines, (c) isotherms, and (d) liquid isocomposition. 156

6.11 Predicted solidification behavior of case NUDS 4R at $900 \mathrm{~s}$ : (a) velocity vectors, (b) streamlines, (c) isotherms, and (d) liquid isocomposition. 157

6.12 Predicted solidification behavior of case NUDS 4R at $1800 \mathrm{~s}$ : (a) velocity vectors, (b) streamlines, (c) isotherms, and (d) liquid isocomposition. 158

6.13 Predicted solidification behavior of case NUDS 4R at $3600 \mathrm{~s}$ : (a) velocity vectors, (b) streamlines, (c) isotherms, and (d) liquid isocomposition. 159

6.14 Species macrosegregation of case NUDS 4R at (a) $1800 \mathrm{~s}$ and (b) $3600 \mathrm{~s}$ 163

6.15 Comparison of overall macrosegregation for static solidification and solidification with rocking . 
Figure

6.16 Predicted solidification behavior of rase NUDS 4R at $605 \mathrm{~s}\left(\theta=-38.4^{\circ}\right)$ :

(a) velocity vectors, (b) streamlines, (c) isotherms, and

(d) liquid isocomposition.

6.17 Predicted solidification behavior of case NUDS 4R at $610 \mathrm{~s}\left(\theta=-22.5^{\circ}\right)$ :

(a) velocity vectors, (b) streamlines, (c) isotherms, and

(d) liquid isocomposition.

6.18 Predicted solidification behavior of case NUDS 4R at $615 \mathrm{~s}\left(\theta=0^{\circ}\right)$ :

(a) velocity vectors, (b) streamlines, (c) isotherms, and

(d) liquid isocomposition.

6.19 Predicted solidification behavior of case NUDS 4R at $620 \mathrm{~s}\left(\theta=22.5^{\circ}\right)$ :

(a) velocity vectors, (b) streamlines, (c) isotherms, and

(d) liquid isocomposition.

6.20 Predicted solidification behavior of case NUDS 4R at $625 \mathrm{~s}\left(\theta=38.4^{\circ}\right)$ :

(a) velocity vectors, (b) streamlines, (c) isotherms, and

(d) liquid isocomposition.

6.21 Predicted solidification behavior of case NUDS 4R at $630 \mathrm{~s}\left(\theta=45^{\circ}\right)$ :

(a) velocity vectors, (b) streamlines, (c) isotherms, and

(d) lianuid isocomposition.

6.22 Teworature histories for case UDS 4R: (a) numerical predictions and

(b) experimental results.

6.23 Predicted temperature histories in the melt of NUDS 4R over one rocking cycle.

6.24 Streamline patterns from NUDS $4 \mathrm{R}$ at (a) $915 \mathrm{~s}$, (b) $930 \mathrm{~s}$, (c) $945 \mathrm{~s}$, and (d) $960 \mathrm{~s}$

6.25 Predicted solidification behavior of case NMDS 1 at $300 \mathrm{s:}$ (a) velocity vectors, (b) streamlines, (c) isotherms, and (d) liquid isocomposition.

6.26 Predicted solidification behavior of case NMDS 1 at $600 \mathrm{~s}$ : (a) velocity vectors,

(b) streamlines, (c) isotherms, and (d) liquid isocomposition.

6.27 Predicted solidification behavior of case NMDS 1 at $900 \mathrm{~s}$ : (a) velocity vectors,

(b) streamlines, (c) isotherms, and (d) liquid isocomposition.

6.28 Predicted solidification behavior of case NMDS 1 at $1800 \mathrm{~s}$ : (a) velocity vectors, (b) streamlines, (c) isotherms, and (d) liquid isocomposition.

6.29 Species macrosegregation of case NMDS 1 at $1800 \mathrm{~s}$ 
Figure

6.30 Temperature histories along vertical thermocouple array located at $\mathrm{x}=38.1 \mathrm{~mm}$ for case MDS 1: (a) numerical prections and

(b) experimental results.

6.31 Comparison of predicted and experimental temperature histories for case MDS 1 at (a) $y=76.2$ and (b) $y=50.8 \mathrm{~mm}$.

6.32 Comparison of predicted and experimental liquid composition of ammonium chloride for case MDS 1.

Appendix

Figure

A.1 Shadowgraph images of UDS with six circular copper fins extending from the heat exchanger at (a) 7, (b) 15, (c) 30, and (d) $60 \mathrm{~min}$

A.2 Top view showing comparison of grain morphology of UDS with and without fins as viewed from the top

B.1 Velocity vectors (left) and streamlines (right) for a fluid body enclosed in a box rocking at one cycle per minute. Conditions shown at (a) $\theta=0^{\circ}$, vertical, (b) $\theta=45^{\circ}$, (c) $\theta=0^{\circ}$, (d) $\theta=-45^{\circ}$ 


\section{LIST OF SYMBOLS}

Symbol

a

c

D

f

g

h

$h_{f}$

J

k

k

K

$\mathrm{K}_{\mathrm{o}}$

L

$\mathrm{M}_{\mathrm{rms}}$

$\mathrm{P}$

$\mathbf{r}$

S

$\mathrm{t}$

$\mathrm{T}$

u

v

\section{Description}

acceleration vector

specific heat

binary mass diffusivity

mass fraction

volume fraction or gravitational acceleration

enthalph

lais:xit heat of fussion

generve surface flux vector

unit vector in $\mathrm{z}$ direction

phase designation or thermal conductivity

permeability

Blake-Kozeny permeability constant

length scale

root-mean-squared of macrosegregation

pressure

position vector

generic source term

time

temperature

$\mathrm{x}$ direction velocity component

y direction velocity component 


\begin{tabular}{|c|c|}
\hline Symbol & Description \\
\hline $\mathbf{v}$ & $v e^{\prime}$ city vector \\
\hline V & volume \\
\hline $\mathrm{x}$ & $x$-coordinate \\
\hline$x_{0}$ & $\mathrm{x}$ displacement to center of rotation \\
\hline y & y-coordinate \\
\hline$Y_{0}$ & y displacement to center of rotation \\
\hline $\mathbf{z}$ & z-coordinate \\
\hline Greek Symbol & Description \\
\hline$\alpha$ & species designation or thermal diffusivity \\
\hline$\beta_{\mathbf{s}}$ & solutal volumetric expansion coefficient \\
\hline$\beta_{\mathrm{T}}$ & thermal volumetric expansion coefficient \\
\hline$\theta$ & angular displacement \\
\hline$\theta_{0}$ & rocking amplitude \\
\hline$\mu$ & dynamic viscosity \\
\hline$v$ & kinematic viscosity \\
\hline$\rho$ & density \\
\hline$\sigma$ & standard deviation of temperature fluctuation \\
\hline$\Psi$ & streamfunction $(\mathrm{kg} / \mathrm{s} \cdot \mathrm{m})$ \\
\hline$\phi$ & generic phase of continuum field variable \\
\hline$\omega$ & rocking frequency \\
\hline$\Delta$ & difference \\
\hline$\Omega$ & rotation vector \\
\hline
\end{tabular}


Subscript symbol

c

e

init

1

liq

m

0

S

Superscript Symbol

a

Nondimensional Number

Le

$\operatorname{Re}$

Ras

$\operatorname{RaT}$

\section{Description}

cold wall

eutectic

initial

liq"id

liquidus

fussion temperature

initial or nominal

solid

Description

species designation

partial property or average quantity

Description

Lewis number, $\alpha / \mathrm{D}$

Reynolds number, (VL)/v

Solutal Rayleigh number, $\frac{g \beta_{S} \Delta \mathrm{f}_{l}^{\alpha} \mathrm{L}^{3}}{v \mathrm{D}}$

Thermal Rayleigh number, $\frac{g \beta_{\mathrm{T}} \Delta \mathrm{T} \mathrm{L}^{3}}{v \alpha}$ 


\begin{abstract}
Using a $2 \% \%$ aqueous ammonium chloride solution as a transparent analog, shadowgraph and dye injection techniques have been employed to observe flow and morphological conditions associated with unidirectional solidification (UDS) from below. When solidification is induced by chilling the solution from below, dendritic crystals which initially form at the cold surface reject lighter, solute deficient fluid, and the attendant instability is manifested by finger-type double-diffusive convection phenomena. As a two-phase (solid/liquid), or mushy, region grows from the bottom surface, perturbations in field variables at the liquidus interface cause localized remelting and the downward development of channels in the mushy region. Solutal plumes which $\bullet$ manate from the channels are characterized primarily by an ascending, oscillatory motion and secondarily by wisps of fluid which detach and descend from bends in the plumes. In time, double-diffusive convection layers also form in the melt. When the solution is chilled from the sides, as well as from the bottom, conditions are influenced by the detachment and subsequent settling of crystals from the sidewall, as well as by plumes emanating from slanted channels which develop in the sidewall mushy region. These plumes enhance the formation of double-diffusive convection layers in the melt, which in turn, influence the motion of plumes emanating from the bottom mushy region.
\end{abstract}

When a slow, oscillatory rocking motion is imposed on the UDS arrangement, thermal and inertially induced convection in the melt act to sweep perturbations in the field variables from the liquidus interface. By removing these perturbations, the downward development of channels in the mushy region is effectively inhibited, and ultimately, the number and severity of freckle- 
type segregates found in the final cast is suppressed. Within the melt, plumes and doublediffusive convection phenomena are eliminated by efficient inertially induced mixing, and quantitative measurements of temperature and species in the melt confirm both the efficient mixing and reduction of overall macrosegregation. When chilling from the side as well as bottom, inertially induced convection mixes the melt and produces a dense slurry. Although channels are eliminated from the bottom wall mushy region, overall heat trarısfer and macrosegregation in the cavity are unaffected by the slow rocking.

Numerical simulations of the experiment qualitatively predict trends in the field variables. The simulations also provide useful insights concerning interdendritic flows and macrosegregation in the form of freckle- and A-type segregates. However, the ability of the model to quantitatively predict the transient behavior of field variables is hampered by the simplifyirig assumptions of negligible turbulence in the flow, a continuous mushy region, twodimensional fiow, and local thermodynamic equilibrium. 


\section{CHAPTER 1 - INTRODUCTION}

\subsection{Solidification of Multicomponent Mixtures}

Systems that undergo phase change from liquid to solid have long been the focus of detailed research, and the solidification of single component systems such as water or pure metals is well understood and readily predicted. In contrast, solidification of multicomponent mixtures consisting of two or more elements is very complicated. Even the solidification of a simple binary alloy (two component mixture) includes, in addition to phase change and heat transfer, the complicating phenomena of mass transfer, doublediffusive convection, and flow through a porous medium. Moreover, these phenomena occur on coupled microscopic and macroscopic scales.

Although very disparate fields. Within the geosciences, considerable attention has been paid to the solidification of aqueous saline solutions. Much work has arisen from oceanographers' interests in melting icebergs and related double-diffusive convection phenomena, as well as the interests of geologists in flow conditions related to magma chambers (Huppert and Sparks, 1984). Crystal growers and engineers investigating thermal energy storage devices have also shown interest in the problem. Probably the most significant research to date has come from the metallurgical sciences, particularly as solidification applies to welding and soldering (Oreper and Szekely, 1984) and the casting of metal alloys (Fisher, 1981). Research has focused both on traditional casting methods involving chilling from all sides of the mold and a specialized casting process known as unidirectional solidification (UDS), which has garnered considerable interest within the past two 
decades. The advantage of UDS is the directional strength of the columnar crystal structure produced when solidification occurs one-dimensionally. Equiaxed structures, for which small crystal grains are arranged in many different orientations, do not provide the same degree of directional strength. One application of UDS technology is in the aerospace industry, where the need for high strength materials has long been associated with the development of high performance gas turbine engines. Although expensive, directionally solidified turbine blades have shown markedly improved thermal creep behavior and strength (McLean, 1983), and engine performance has benefited from the high-strength, light-weight, super alloy turbine blades grown by directional solidification. Unfortunately, many alloy casts often suffer from defects which develop during the solidification process.

Free convection plays an important role in the solidification of off-eutectic binary alloys. Viskanta (1985) gave a detailed review of convection influenced solidification, while Huppert (1990) reviewed recent work into solidification of binary mixtures. The major complication arising from solidification of alloys is that, in addition to the expected thermal effects, buoyancy is also produced by differences in fluid composition resulting from the solidification process. As an alloy is solidified, the generation of solutally buoyant fluid can act to augment or suppress thermal buoyancy, and the combination of both effects can lead to complex flows referred to as double-diffusive convection. One problem associated with the quality of alloy casts is termed macrosegregation and has been attributed directly to the convective transport of species during the solidification process. Macrosegregation is manifested by large scale redistribution of melt components to localized areas within the cast. These areas of segregation represent weak regions which are usually detrimental to the quality of the final product.

Within the past ten years, the coupling of segregation to free convection has been extensively studied and clarified. Recent numerical developments have led to a detailed 
continuum model which, in combination with related experiments, has provided a deeper understanding of the coupling between convection and macrosegregation. In fact, the technology has advanced to the point where research efforts are beginning to explore control schemes which reduce or eliminate segregates altogether. Increasing thermal gradients within the melt, adding additional components to a melt, casting ingots in space, or control of melt convection with externally applied body forces are all schemes which have been used to control segregation. These schemes have had varying degrees of success.

\subsection{The Macroscopic Physics of Solidification of a Binary Alloy}

While freezing of a pure substance is characterized by the advancement of a distinct line separating the all liquid from the all solid regions of the cast, solidification of a binary mixture generally results in a more complex growth front. Due to morphological instabilities which develop along the growth front of a binary cast, three distinct phases exist within the system. As in the freezing of a pure substance, all solid and all liquid regions exist, but under off-eutectic conditions, a third region forms. Commonly referred to as a mushy zone, this third region, consisting of solid dendritic crystals and interstitial (interdendritic) fle:d, forms essentially a porous, crystalline structure. The concentrations of the solid and liquid portions of the mushy region are thermodynamically dependent upon the temperature at which the crystals form. However, the composition of crystals that form within the mushy zone, as well as that of the interdendritic fluid which is rejected by the growing crystals, differs from that of the original melt. Because this interdendritic fluid is compositionally different, solutal buoyancy can develop, ultimately having a strong influence on convective transport within the cast.

During the freezing process, thermal gradients in the melt produce density differences which, under the influence of gravity, promote free convection. However, 
when solidification of an off-eutectic binary alloy occurs along a chilled side wall, lighter interdendritic fluid may be rejected into the interstices of the mushy region and advected upward. Rising through the sidewall mushy region and eventually exiting into the melt, a top layer of thermally heavier (colder) and compositionally lighter fluid is established, creating conditions conducive to the formation of double-diffusive convection cells within the liquid region. The upwelling of fluid erodes channels within the mushy region, which are subsequently overgrown by the advancing solid region, thereby forming slanted pencil-like structures in the final cast. A classic example of macrosegregation, these pencil-like regions are referred to as A-type segregates.

When cooling occurs from the bottom surface only, as in UDS, a different form of segregation can develop. An off-eutectic melt chilled from below will again form a solid, liquid, and mushy region, but if the colder interdendritic fluid becomes solutally lighter than the overlying fluid, a double-diffusive instability can occur, promoting convection within the mushy region. Owing to a large Lewis number, a parcel of interdendritic fluid which is perturbed upwards will be heated by the surrounding fluid while maintaining its solutal composition. If thermodynamic equilibrium is maintained, localized melting can be induced by the displaced parcel, and if a continuous upflow of fluid is maintained at one location for an extended period of time, the melting will produce a vertical hole (Worster, 1991). This hole or channel acts like a chimney, transporting light, interdendritic fluid from the mushy region into the melt. The advancing solid region eventually overgrows the vertical channels, filling them with material which differs compositionally from that of the surrounding areas of the cast. Examining a cross-section of the cast solid, the channel segregates appear as blemishes or freckles within the surrounding columnar crystal structure. The term freckle-type segregate describes this form of macrosegregation. These freckle segregates which develop in directionally solidified casts weaken the final product and are generally detrimental to performance 
(Giamei and Kear, 1970). Though most destructive, freckles are not the only form of segregation found in UDS casts.

During solidification of a binary alloy for which lighter, solute deficient fluid is continuously being rejected by the advancing mushy region, upward advection of this fluid stratifies the melt. Therefore, when completely solidified, the cast is solute rich in its lower regions and solute deficient in upper regions. This type of segregation is referred to as banded segregation and is present in any cast produced from a melt which is compositionally stratified.

\subsection{Literature Review}

\subsubsection{Experimental Work}

The study of macrosegregation associated with binary alloy solidification has focused on low temperature metal alloys (primarily lead-tin) and translucent aqueous saline solutions. Traditionally, metallurgists have studied macrosegregation using simple binary alloys. In an early work, Mehrabian et al. (1970b) solidified aluminum-copper from a side wall measuring melt temperature and gaining macrosegregation information through post-cast processing. More recently, Ridder et al. (1981) solidified lead-tin using electroslag remelting, and Wang et al. (1988) investigated freckle-type segregation in UDS casts of lead-tin. However, the insight which led investigators to discover the primary cause of macrosegregation (convective transport in the mushy region and the melt) did not initially come from work with metal systems.

Working with aqueous ammonium chloride $\left(\mathrm{NH}_{4} \mathrm{Cl}-\mathrm{H}_{2} \mathrm{O}\right), \mathrm{McDonald}$ and Hunt (1969) were the first to report the formation of A- and V-segregates in a side chill cast. A popular low temperature analog to metal alloys (Jackson et al., 1966), aqueous $\mathrm{NH}_{4} \mathrm{Cl}$ is often utilized to study solidification of binary mixtures. In addition to freezing dendritically, the analog is transparent, thus allowing observation of convective fluid 
motion during solidification. Also using aqueous $\mathrm{NH}_{4} \mathrm{Cl}$, Coplcy et al. (1970) showed that, under certain conditions in the UDS system, large vertical channels develop in the mushy region and become the source of freckle segregates in the final casting. However, for directional solidification of $\mathrm{NH}_{4} \mathrm{Cl}-\mathrm{H}_{2} \mathrm{O}$ systems, freckle type segregation has been reported only for a narrow band of mixture compositions from approximately $26 \%$ to $34 \% \mathrm{NH}_{4} \mathrm{Cl}$ (Sarazin, 1990). In a very clever set of experiments, Sample and Hellawell (1982, 1984) determined that channel formation depends strongly on perturbations associated with lighter fluid at the liquidus front. Worster (1991) provided physical interpretations of the formation of channels within the mushy region, while Neilson and Incropera $(1991,1992 a)$ obtained detailed numerical simulations which elucidated key physical phenomena and their relationship to channel and freckle formation. The dependence of macrosegregation on convection in metal alloys has been verified using radioactive dye tracers for both side chill and UDS arrangements (Stewart and Weinberg, 1972; Streat and Weinberg, 1974).

Several investigators have studied salt systems chilled from a side wall (Asai and Muchi, 1978; Szekely and Jassal, 1978; Murakami and Okamoto, 1984; Beckermann and Viskanta, 1988; Thompson and Szekely, 1988; Christenson and Incropera, 1989a; Nishimura et al., 1992). Asai and Muchi (1978) chilled an aqueous $\mathrm{NH}_{4} \mathrm{Cl}$ solution from both sides in a rectangular cast and used a potassium permanganate dye to trace fluid motion in the side wall mushy region. Using similar test conditions, Murakami and Okamoto (1984) documented the ejection of crystals or a slurry into the melt from a side wall mushy region. Chilling aqueous sodium carbonate from a side wall, Nishimura et al. (1992) used temperature sensitive liquid crystals to track flow conditions and isotherms in the melt. Christenson and Incropera (1989c) studied the convective transport of aqueous sodium carbonate in a cylindrical annulus. Using shadowgraph and fluorescein dye injection at the liquidus interface, they revealed the existence of solutal and thermal 
plumes as well as double-diffusive convection cells. More recently, research has concentrated on the UDS process.

McCay et al. (1989), McCay and McCay (1990), and Hopkins et al. (1990) used Schlieren photography and particle tracking to study Rayleigh-Benard type instabilities which preceded plume development. Okumura et al. (1989) and Sarazin (1990) used shadowgraph techniques to show the evolution from salt fingers to channels in the UDS of aqueous $\mathrm{NH}_{4} \mathrm{Cl}$. Sarazin also obtained temperature: and composition measurements in the melt for the UDS of aqueous ammonium chloride, as did Neilson (1991). The most complete photographic study to date has been performed by Chen and Chen (1991), who also used shadowgraph techniques to reveal the nature of salt fingering in the early stages of solidification and the development of plumes in subsequent stages. Although Chen and Chen restricted their experiments to a single composition ( $\left.26 \% \mathrm{NH}_{4} \mathrm{Cl}\right)$, they did consider a range of cold wall temperatures $\left(-31.5^{\circ} \mathrm{C}<\mathrm{T}<11.9^{\circ} \mathrm{C}\right)$. Directional solidification from the top wall has also been investigated using aqueous isopropanol (Kerr et al., 1990) and aqueous $\mathrm{NH}_{4} \mathrm{Cl}$ (Cao and P.julikakos, 1990). A detailed review of the solidification process has recently been written by Huppert (1990).

\subsubsection{Analytical and Numerical Investigations}

In an effort to better understand or predict macrosegregation in alloy ingots, several models of the solidification process have been proposed. The first such attempts appear to have come in the late 1960's (Flemings and Nereo, 1967; Flemings et al., 1968; Flemings and Nereo, 1968). These first simple models were limited in their scope and capabilities. They predicted macrosegregation in the mushy region by considering convection due only to thermal contraction (shrinkage) and required prescription of the solidification rate, nature of the interdendritic flow, and local temperature gradients. The model was later improved by treating the mushy region as a Darcian porous medium and 
including convection due to buoyancy as well as shrinkage (Mehrabian et al., 1970a; Kou et al., 1978). However, it still required prescription of solidification rate and temperature gradients. An attempt to couple the momentum and energy equations within the mushy region was carried out by Fujii et al. (1979), but the resulting equations were uncoupled to both the liquid and solid regions, and knowledge of the size and shape of the mush was required. Ridder et al. (1981) coupled the two-dimensional flows within the melt and the mush, but their model again required apriori knowledge of the size and shape of the mushy zone. Szekely and Jassal (1978) were the first investigators to consider both the momentum and energy equations in the solid, liquid, and mush; however, the model did not account for the transport of species. Therefore, convection due to solutal buoyancy and macrosegregation could not by predicted. A number of continuum or volumeaveraging models that account for momentum, energy, and species transfer began to appear in the late 1980's (Bennon and Incropera, 1987a; Voller and Prakash, 1987; Beckermann and Viskanta, 1988; Ganeson and Poirer, 1990).

The major advantage to a continuum or volume-averaging approach for modelling solidification of binary mixtures is the inherent ability of the model to predict solutal/thermal convection and macrosegregation simultaneously, without the need to prescribe variables or conditions in the cast. All of the continuum models, though slightly different in their development, yield similar results and predict similar phenomena (Prescott et al., 1991). Early simulations of the freezing of aqueous ammonium chloride in a rectangular cavity from a side wall showed good qualitative agreement with experiments and captured key solidification phenomena like doublediffusive convection and macrosegregation in the mushy region (Bennon and Incropera, 1987b; Christenson et al., 1989b). Amberg (1991) has also found good agreement with experiments when simulating the casting of carbon steel, and Prescott and Incropera (1992a) have shown similar results modelling lead-tin systems. For UDS of aqueous 
$\mathrm{NH}_{4} \mathrm{Cl}$, Neilson and Incropera $(1991,1992 a)$ have quite effectively captured the formation of channels and resultant macrosegregation (freckles). Similarly, Felicelli et al. (1991) have successfully predicted freckle formation in the UDS casting of lead-tin.

Most recently, Neilson and Incropera (1992b) acknowledged that a twodimensional model did not accurately capture the three-dimensional nature of freckling and developed a fully three-dimensional solidification model. Their findings suggested that existing 2-D models accurately capture the major features of freckling, and considering the computational cost benefits, provide useful and efficient engineering tools.

\subsubsection{Macrosegregation Control Schemes}

The ultimate goal of research into the solidirication of binary alloys is to improve casting products by reducing or eliminating macrosegregation, and several schemes to control macrosegregation have been proposed. Early investigators have determined from stability analyses that sharp thermal gradients within the mushy region of a UDS cast will eliminate freckles (Copley et al., 1970). However, such extreme casting conditions may not be practical for commercial foundries. Another proposal involved adding an additional component to an alloy which would change the phase diagram ard eliminate or reverse solutal buoyancy, the major contributor to macrosegregation (McDonald and Hunt, 1970). Although not a panacea, such techniques have shown promise in certain circumstances. The use of magnetic fields to dampen convective motion during solidification of metal alloys has also been investigated (Prescott and Incropera, 1992a). Probably the most effective, albeit expensive, process which has been proposed is the casting of super alloys in space. In theory, solidification in the absence of gravity would eliminate free convection and, hence, eliminate macrosegregation (McCay and McCay, 1988). Unfortunately, this solution is far from being cost effective. A fruitful ground- 
based answer to the problem of macrosegregation seems to be in actively controlling convective motions within the melt during solidification.

In an important set of experiments, Sample and Hellawell (1982) found that by slowly rotating a tilted cast during solidification, freckles could be eliminated. Their original conjecture was that changes in the gravity vector with respect to the mushy region would sufficiently disturb the flow of interdendritic fluid discouraging a continuous upflow at one location. If the upflow was distributed over many dendrite crystals, no single cadre of crystals could erode or melt thereby forming a channel. However, they later changed their hypothesis to say that the elimination of freckles was not due to the change of gravity but rather to sweeping of the liquidus interface (boundary hetween the mushy and liquid regions) by fluid in the melt (Sample and Hellawell, 1984). They concluded that perturbations in density along the liquidus interface were responsible for channeling and that smoothing or removing these perturbations would eliminate channeling. Following Sample and Hellawell's lead, Neilson and Incropera chose to apply only the sweeping principle in the UDS casting of aqueous $\mathrm{NH}_{4} \mathrm{Cl}$.

Investigating the unidirectional solidification of $\mathrm{NH}_{4} \mathrm{Cl}$ in a vertical, axisymmetric mold, Neilson and Incropera (1992c, 1992d) numerically and experimentally agitated the cast by means of slow intermittent rotation. By intermittently starting and stopping the cast, shear layers developing on the liquidus interface during start-up would sweep density perturbations from the mush and suppress channeling. Their experiments did suppress freckling in the interior region of the cast, but channels did still appear around the periphery.

\subsection{Objectives of the Current Study}

The primary objective of the present study is to attempt to use a simple rocking scheme to reduce macrosegregation associated with the solidification of a binary alloy. It 
is hoped that by slowly oscillating a solidifying binary alloy of aqueous ammonium chloride, sweeping of the liquidus interface by both inertial advection and thermal buoyancy will eliminate channeling. In addition, the effect of oscillation on a mold chilled from the sides as well as the bottom will be addressed. To provide a standard for assessing the effect of rocking, casting of static molds is also studied.

The present study expands on previous work by providing an in-depth examination of flow conditions affecting cast quality under static conditions. It also offers new insights into the process of segregate formation, the importance of slurries in castings, and double-diffusive phenomena associated with concurrent UDS and side chill processes. Using shadowgraph, particle tracking, and dye injection directly into the mushy region, previously unreperted phenomena such as the UDS slurry zone, doublediffusive convective cells in the UDS environment, and plume instabilities are shown to exist. In addition, temperature and concentration characteristics of the solidification process will be documented.

Finally, a numerical analysis based on Bennon and Incropera's original continuum model will be used to simulate the experimental conditions. The model will be used primarily as a tool to elucidate otherwise undetectable phenomena present in the experiments, such as macrosegregation and the nature of interdendritic flow. Also, comparisons between the experimental and numerical results will be made in order to help validate the continuum model. 


\section{CHAPTER 2 - EXPERIMENTAL PROCEDURES}

\subsection{Test Sections}

In order to study a wide range of solidification phenomena, two test sections were constructed, each physically modelling a unique casting process. Although both sections were dimensionally identical, test section I was designed for uaidirectional solidification, while test section II was designed for casting from the sides ; well as the bottom. In designing the test sections, both the size and shape were carefully considered. To simplify handling and manageability, as well as to minimize the volume of phase change material, the test sections were kept to the smallest possible size, while still allowing macrosegregation and channeling to occur. A rectangular shape was chosen to allow easy observation and photographic access. The rectangular shape also simplified numerical simulations of the experiments by permitting the governing equations to be written in Cartesian coordinates. The test sections measured $101.6 \mathrm{~mm}$ high by $101.6 \mathrm{~mm}$ wide by $25.4 \mathrm{~mm}$ deep and were intentionally made shallow in the direction of viewing to minimize the three-dimensionality of the process (Fig. 2.1).

The bottom wall of test section I consisted of a single copper heat exchanger, whilp all other walls were made from a clear polycarbonate (Lexan). The lid to the test section contained a small hole and a supply tube, through which the test section was filled with solution before each experiment. Since aqueous $\mathrm{NH}_{4} \mathrm{Cl}$ contracts considerably during solidification, the tube slowly supplied fluid, as needed, to the test section over the course of an experiment. In test section II, the Lexan end pieces were replaced with copper heat exchangers, thereby permitting solidification from the sides as well as from 


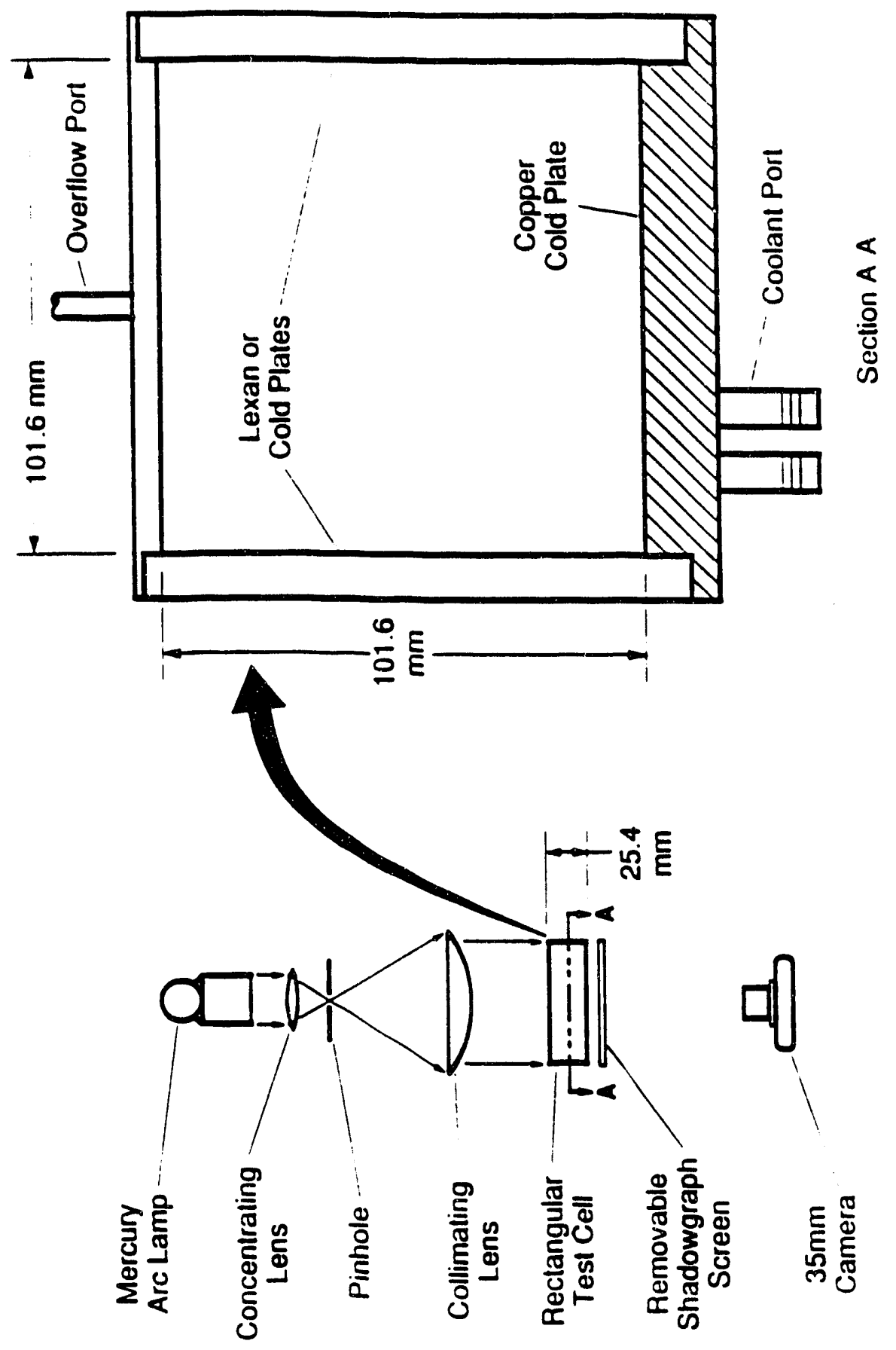

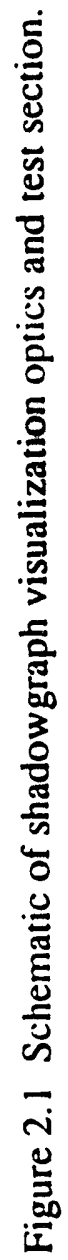


the bottom. The surface temperature of the heat exchangers was uniform to within $0.5^{\circ} \mathrm{C}$ during an experiment, and the termperature asymptotically approached the desired cold wall temperature during the course of an experiment. However, by the end of an experiment, the heat exchanger surface was only to within $4^{\circ} \mathrm{C}$ of the desired cold wall value.

To prevent corrosion, the copper heat exchanger surfaces were protected with a Teflon based PTFE 1201 coating on the order of $25 \mu \mathrm{m}$ thick. Applied locally by Great Lakes Chemical Company, the coating is resistant to corrosion and is commonly used to protect metal parts used on ocean drilling rigs. Both test sections were chilled with a Neslab ULT-80DD constant temperature bath, and for most experiments, the walls were chilled to $-30^{\circ} \mathrm{C}$. However, during dye injection experiments, the cold walls were maintained at $-15{ }^{\circ} \mathrm{C}$ and $-20{ }^{\circ} \mathrm{C}$ for test sections I and II, respectively. The higher temperatures were needed to keep the dye injection ports from freezing shut and did little to alter basic flow conditions. The coolant fluid was ethyl alcohol 190. Both test sections were well insulated during experimentation, with the insulation briefly removed to photograph flow conditions. When performing an experiment, the binary material was equilibrated to an . serature of $25^{\circ} \mathrm{C}$ and became quiescent within the test section. To start an 6. ent, the valve on the cold bath was impulsively opened allowing cold fluid to rush in and quench the heat exchanger. During rocking experiments, the agitation was started well before application of the cold fluid to insure advective conditions were present at the beginning of solidification.

\subsection{Rocking Mechanism}

The primary focus of the present study is to investigate the effects of a slow rocking motion on solidification. Early in the project, it was determined that the rocking motion of the test section should be relatively slow and that the motion should closely 
approximate a sinusoid. In general, the rocking criteria were chosen so that comparisons could be made with previous agitation conditions studied by Sample and Hellawell (1982), in which they suppressed channeling by spinning a tilted $\left(30^{\circ}\right)$ UDS test section at $5 \mathrm{rpm}$. Therefore, a maximum amplitude of $45^{\circ}$ was chosen to take full advantage of the liquidus front sweeping produced by thermal buoyancy effects. The rocking rate needed to be slow enough to avoid shearing the crystals at the liquidus front while fast enough to sweep perturbation from the liquidus front. A rocking rate on the order of one cycle per minute, based on the work of Sample and Hellawell, was then targeted. The sinusoidal rocking was chosen as the simplest first step toward finding an optimal rocking scheme to reduce macrosegregation. However, generating a perfect sine wave with a mechanism was not trivial.

It has been shown (Midha et al., 1985; Farhang et al., 1987) that in a four-bar linkage, as the ratios of the length of the driver bar to the lengths of the remaining linkages approach zero, the motion of the follower bar approaches a sinusoid. Moreover, a near sinusoidal motion can be achieved with a four-bar mechanism using reasonably sized linkages. Applying a graphical four-bar linkage design technique, Norton et al. (1992) designed an appropriate mechanism for this particular problem. Figure 2.2 shows the resulting design and the predicted performance. The linkage consists of a follower-todriver ratio of 4:1 and a coupler to driver ratio of 8:1. It produces an output amplitude of $15^{\circ}$ in the follower. As the driver moves in a circular fashion, the coupler translates the motion to the follower, which responds by rotating back and forth around its base. The designed mechanism produces a sinusoidid motion to withing $5 \%$ of a true sine wave, and for objectives of the present study, the sinusoidal performance of the design, although not exact, is certainly acceptable.

In the design of the table top rocking apparatus (Fig. 2.3), the decision was made to separate the four-bar linkage from the test section rocking assembly. Therefore, 


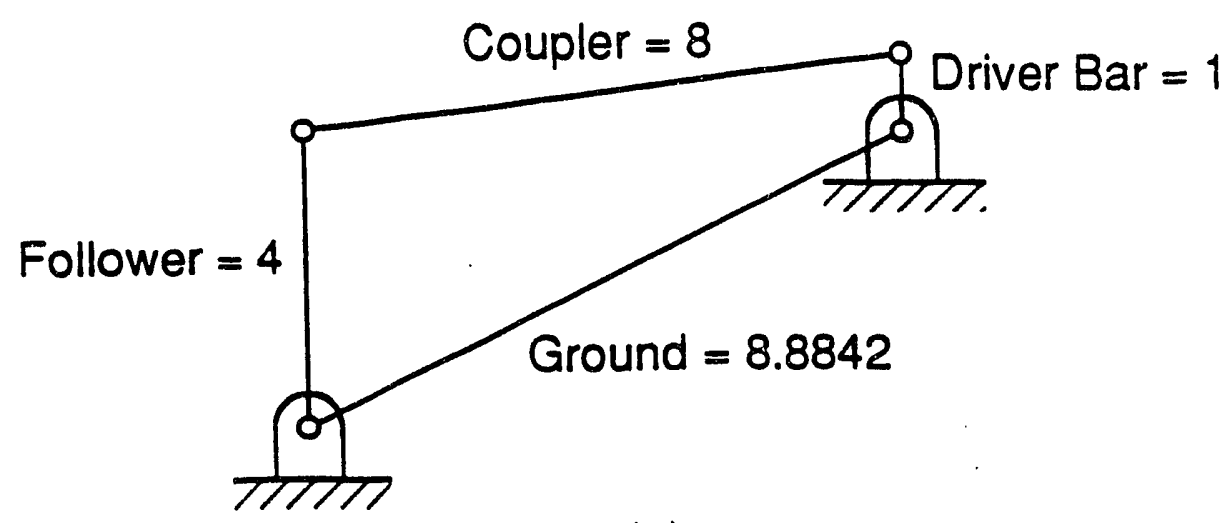

(a)

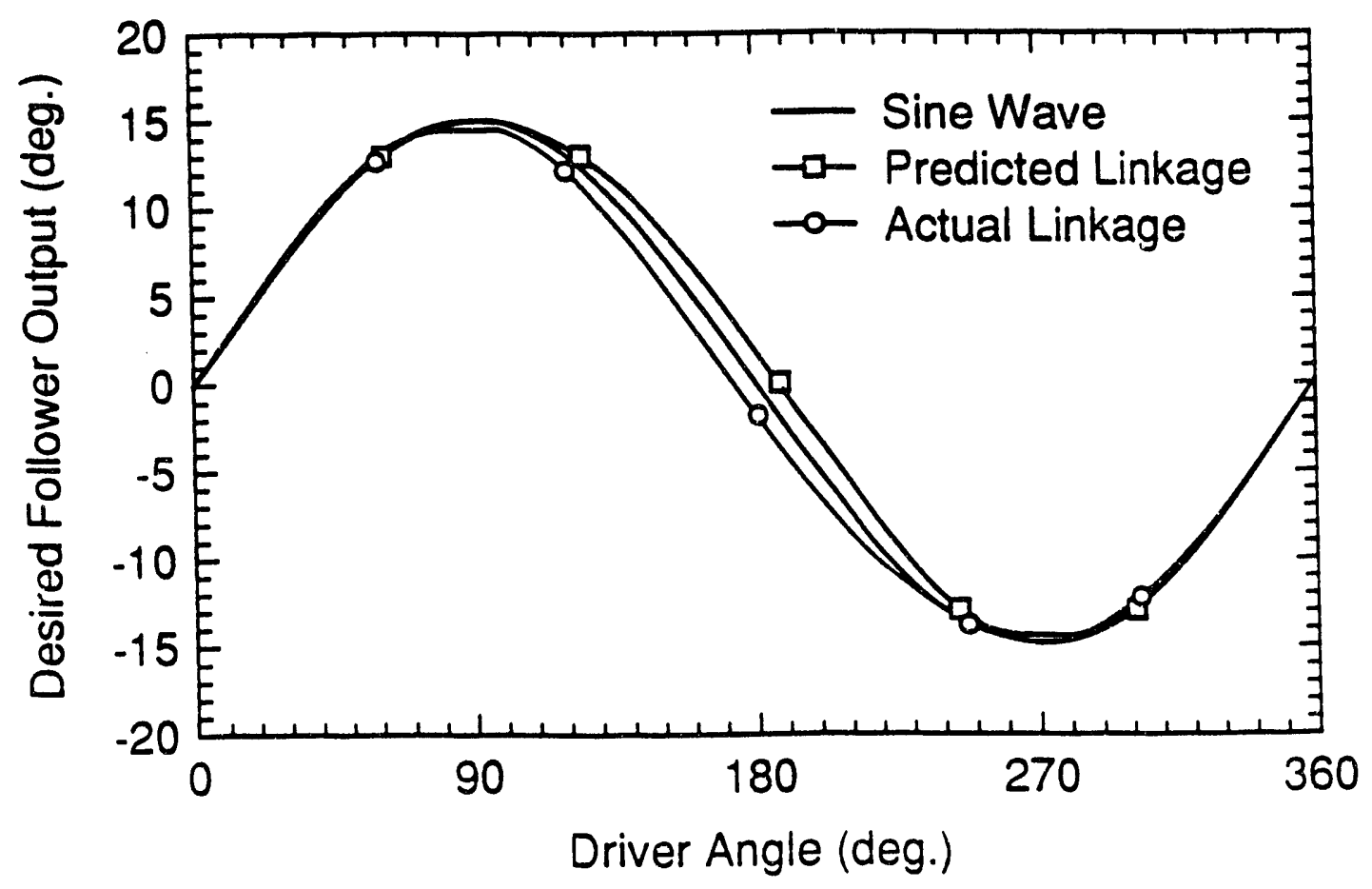

(b)

Figure 2.2 Design of four-bar linkage showing (a) dimensions of each bar and (b) predicted and actual linkage performance. 
Test Section Gearbelt Four-Bar Linkage

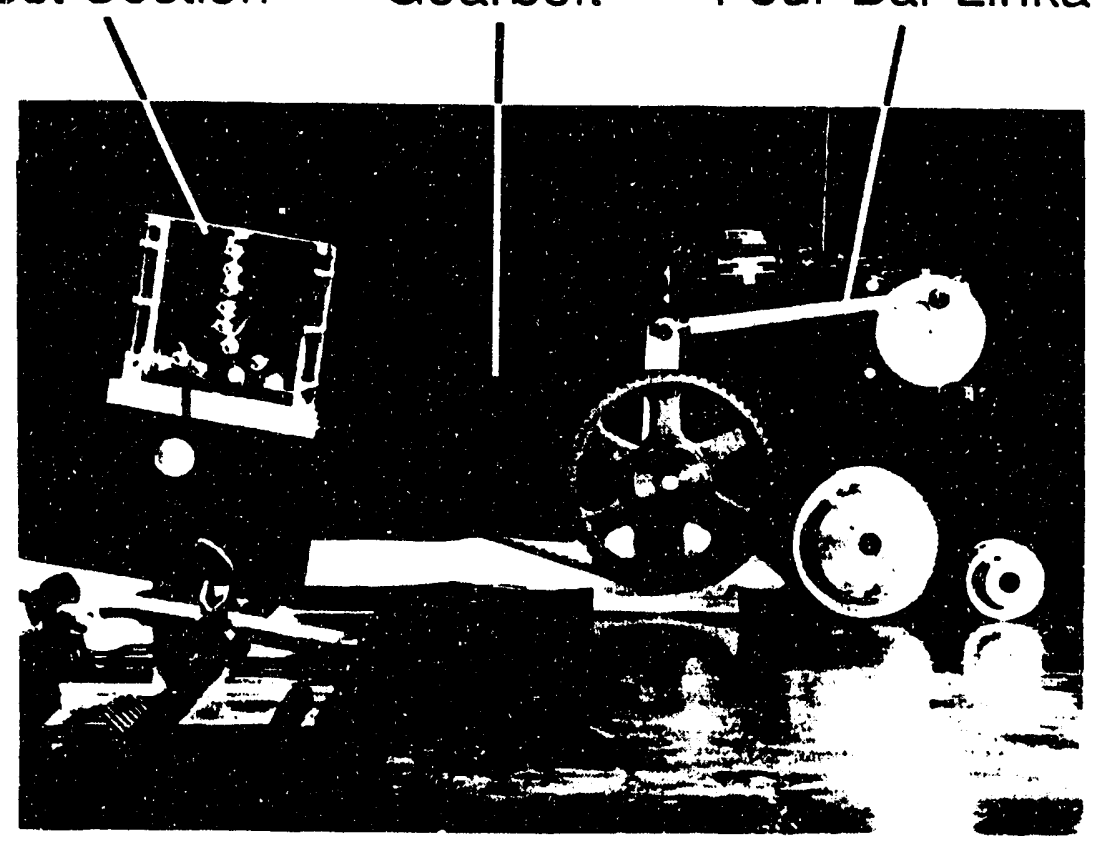

(a)

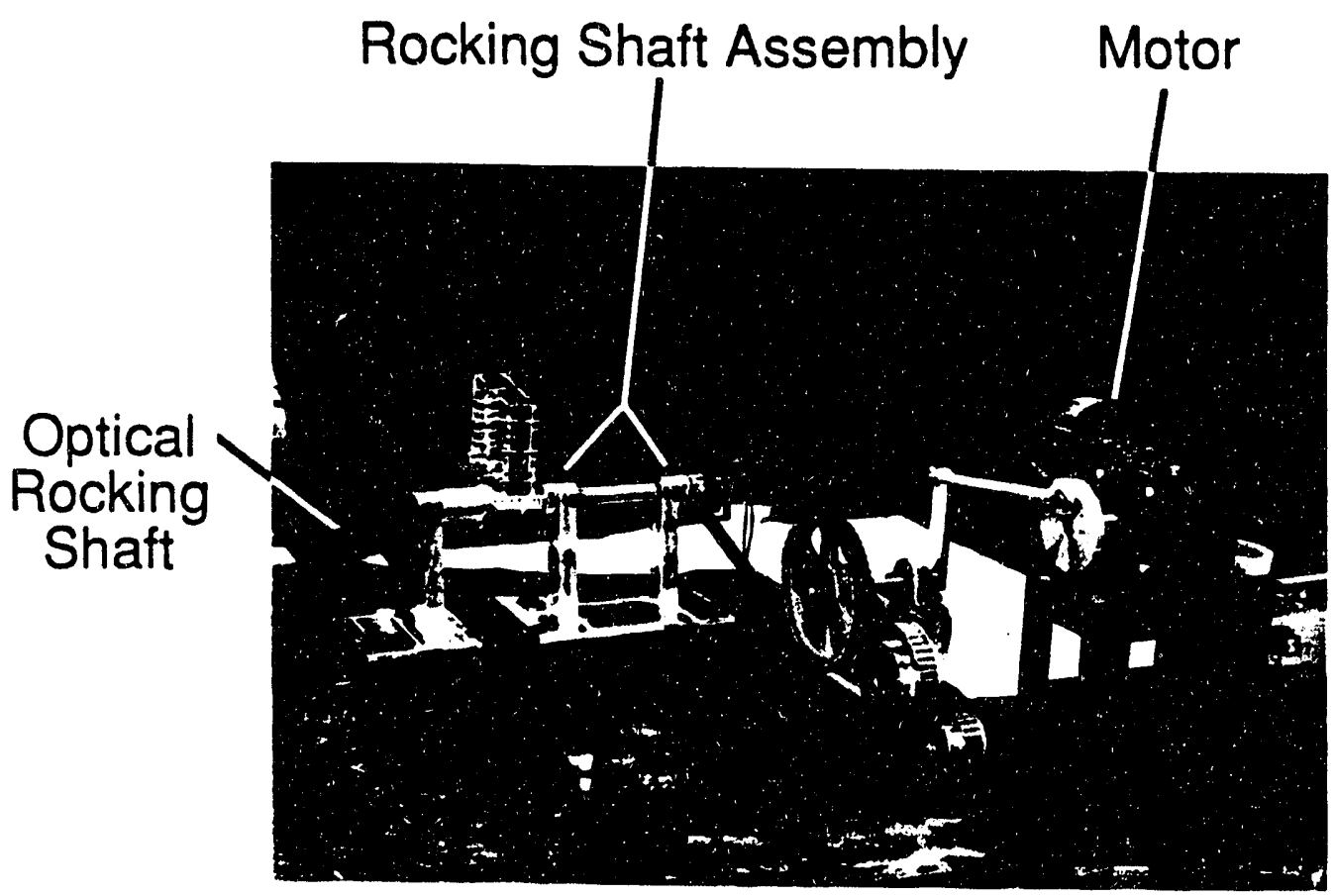

(b)

Figure 2.3 Complete table-top experimental apparatus. 
additions or changes could be made to either part without effecting the other. The driver bar of the four-bar linkage was an aluminum wheel which was mounted on the output shaft of a shunt wound DC motor. Manufactured by the Bodine Electric Company (No. 581 ), the motor was rated at $1 / 15$ horsepower and had a gear ratio of 1080:1. Motor speed was controlled with a Bodine No. 903 variable speed controller. The length from the motor output shaft to the location where the follower bar is attached to the driver wheel (effective driver bar length) was chosen to be $31.8 \mathrm{~mm}$, and all other links were scaled accordingly. The coupler consisted of a long aluminum rod with rod end bearings fitted into the ends. The follower was machined from aluminum and pivoted around its base with a $15^{\circ}$ amplitude. Pillow blocks at the follower base acted to transmit motion to a toothed pulley, which, in turn, transferred motion to the test section rocking assembly.

A $0.107 \mathrm{~m}$ pitch toothed gearbelt transmitted power from the toothed output pulley on the four-bar linkage to a similar pulley placed on the test section rocking shaft. The rocking shaft assembly was made primarily from machined aluminum and consisted of a large flat base and two robust pillars which held the rocking shaft $0.152 \mathrm{~m}$ above the table. The rocking shaft, made from $25.4 \mathrm{~mm}$ diameter drill rod, rotated within the pillars with the aid of Thompson Nyloner bearings distributed locally by Bearings, Inc. The test section was held to the rocking shaft with a specially built aluminum cradle. The cradle held both test sections interchangeably such that the upper surface of the bottom cold plate of a particular test section was always $41.3 \mathrm{~mm}$ above the rocking axis. Because the maximum amplitude of the four-bar linkage was only $15^{\circ}$, pulley ratios between the linkage and rocking shaft of $3: 1,2: 1$, and $1: 1$ were used to achieve rocking shaft amplitudes of $45^{\circ}, 30^{\circ}$, and $15^{\circ}$ respectively. The gearbelt and pulleys were supplied by Browning Inc. A rocking optical assemble, which attached directly to the primary shaft, was also constructed. The optical assembly could accommodate a $35 \mathrm{~mm}$ still camera or a large video tape recorder to record conditions within the test section. 
The rocking angle of the test section was measured with an angular potentiometer, and the overall performance of the four-bar linkage, though not perfect, was acceptable (Fig. 2.2). The maximum amplitude of the rocking shaft was only about $43.5^{\circ}$. This smaller than designed value was attributed to very slight play present in the components of the four-bar linkage and to friction present in the nylon bearings which stopped the shaft prematurely at the maximum amplitudes.

\subsection{Phase Change Material}

Because its liquidus temperature of $18.6^{\circ} \mathrm{C}$ is well below room temperature, an aqueous solution of $27 \% \mathrm{NH}_{4} \mathrm{Cl}$ was chosen for all experiments. This concentration simplified handling of the solution and prevented unwanted crystal nucleation before an experiment. Aqueous ammonium chloride has a eutectic composition of $\mathrm{f}_{e}^{\alpha}=0.197$ at a temperature of $\mathrm{T}_{\mathrm{e}}=-15.4^{\circ} \mathrm{C}$, and the $\mathrm{NH}_{4} \mathrm{Cl}-\mathrm{H}_{2} \mathrm{O}$ equilibrium phase diagram is shown in Figure 2.4. According to the phase diagram, when a $27 \% \mathrm{NH}_{4} \mathrm{Cl}-\mathrm{H}_{2} \mathrm{O}$ solution is chilled, pure ammonium chloride crystals solidify, first rejecting a water-rich interdendritic fluid which is solutally buoyant, thereby promoting double-diffusive convection. This particular binary alloy was chosen for a number of reasons: 1) the transparency of the system facilitated detailed flow visualization and observation of the phase change process; 2) aqueous ammonium chloride is similar to metal systems in both its dendritic crystal growth and its tendency to form channels and promote macrosegregation; 3) the availability of thermophysical data allows numerical simulation of the solidification; and 4) compared to metal alloys, aqueous ammonium chloride is relatively safe and easy to work with in a bench-top application. The one major disadvantage of the material is its extreme corrosiveness to copper and other metals; hence all heat exchanger surfaces must be protectively coated (see section 2.1 ). To reduce void formation during an experiment, the $\mathrm{NH}_{4} \mathrm{Cl}-\mathrm{H}_{2} \mathrm{O}$ solution was de-gased before freezing. 


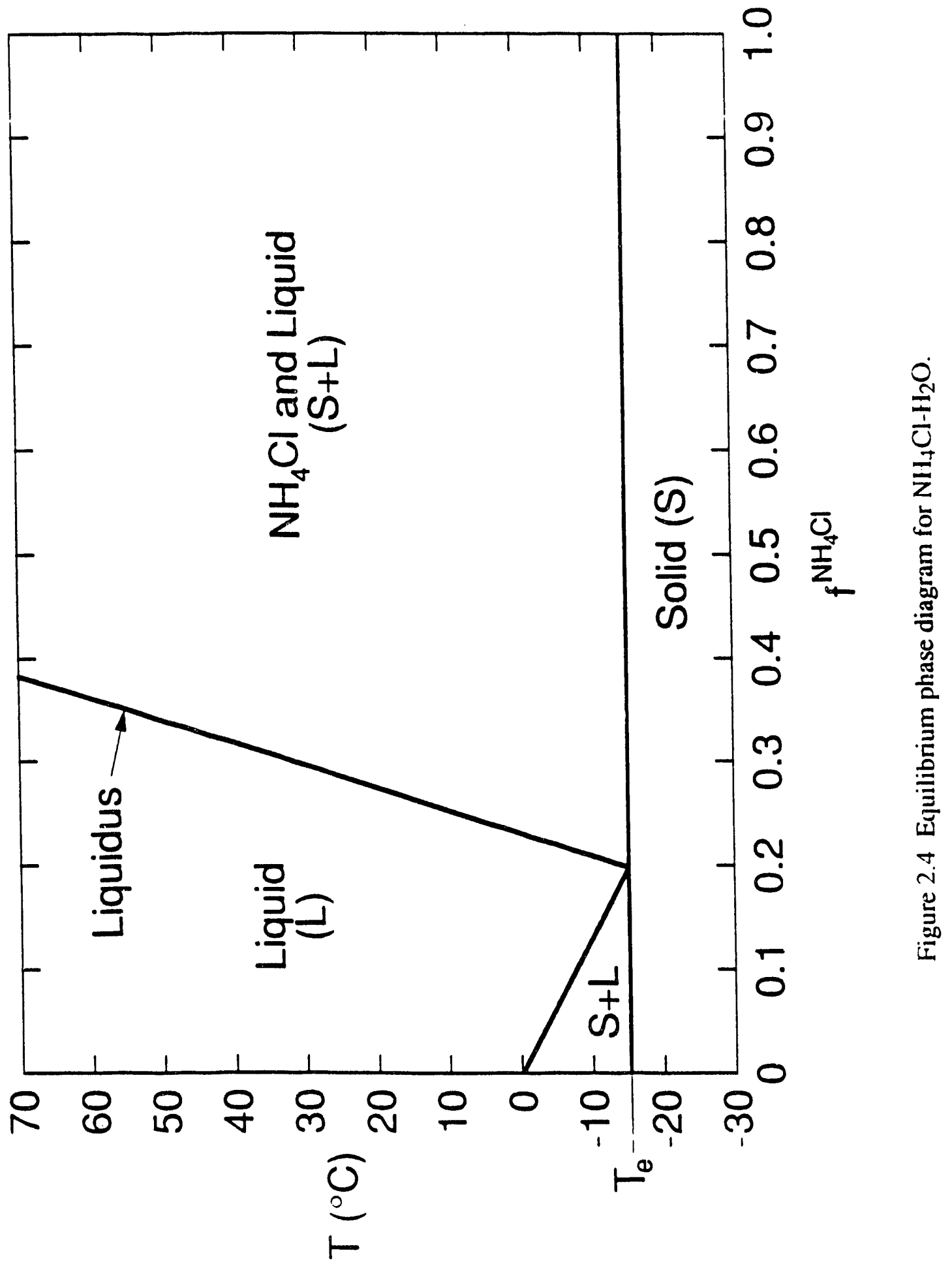


2.4 Qualitative and Quantitative Measurements

\subsubsection{Flow Visualization Techniques}

The primary flow visualization tool was the shadowgraph technique (Goldstein, 1983). As a collimated beam of light passes through the test section (Fig. 2.1), it is deflected in accordance with existing density gradients and shadows are cast on an imaging screen. Because of large density differences in a solidifying salt system, the shadowgraph is well suited for identifying plumes and double-diffusive convection. Shadowgraph images were captured photographically using 400 ASA black and white film, with a $1 / 15 \mathrm{~s}$ exposure time and $\mathrm{f} 4$ aperature. However, the shadowgraph is limited by virtue of providing a two-dimensional view of what are essentially three-dimensional phenomena. Hence, dye injection was also used to view plumes and double-diffusive cells.

To visualize flow patterns emanating from the mushy region, a dye solution of fluorescein disodium salt was injected directly into the mushy region. Before a particular experiment, four 16 gage hypodermic needles were strategically placed around the test section. The needles were filled with a eutectic concentration $\left(19.6 \% \mathrm{NH}_{4} \mathrm{Cl}\right)$ of solution colored with the fluorescein dye. Solidification was then begun and allowed to continue unimpeded until the mushy region had overgrown the needle locations and well established channels and plumes had developed. The dyed eutectic was then slowly released into the dendritic structure and allowed to follow the flow of interdendritic fluid exiting the channels. Concurrently the dye was fluoresced with either a source of black, fluorescent light or a sheet of argon-ion laser light.

Finally, particle tracking was utilized to further reveal flow patterns within the melt. Among the many available flow tracers, small pliolite particles having nearly the same density as the $\mathrm{NH}_{4} \mathrm{Cl}$ solution worked best and were used for all particle tracking 
experiments. The particles were illuminated with a thin sheet of white light from a mercury vapor lamp.

\subsubsection{Temperature Measurement}

In all experiments, type $\mathrm{T}$ (copper-constantan) thermocouples, calibrated to an accuracy of $\pm 0.05^{\circ} \mathrm{C}$, measured temperature characteristics within the test apparatus. For measuring temperatures in the melt, the thermocouples were housed in 16 gauge (1.59 $\mathrm{mm}$ ) stainless steel probes which were strategically placed with swagelok fittings into the side wall of the test section. Insertion from the side wall was chosen to minimize fin probe effects resulting from strong vertical thermal gradients in the melt.

In test section I, nine probes were placed at heights above the cold wall of 6.35 , $12.7,19.05,25.4,38.1,50.8,63.5,76.2$, and $88.9 \mathrm{~mm}$. Actual probe locations were estimated to be within $\pm 1 \mathrm{~mm}$ of the prescribed locations. The upper five thermocouples were placed on the test section centerline, while the remaining four were positioned at staggered horizontal locations, none being aligned with each other (Fig. 2.5). It was observed early in the project that probes placed near the bottom of the test section would induce channels in the mushy region, and the resulting channels would release extremely cold laminar plumes of fluid into the melt. The staggered array pattern was therefore chosen to obtain a better indication of bulk melt temperatures.

Thirteen thermocouple probes measured temperature distributions in test section II. The probe locations were chosen such that one vertical and two horizontal temperature profiles could be determined. The vertical array, placed $38.1 \mathrm{~mm}$ from the side wall heat exchangers, consisted of seven probes located 12.7, 25.4, 38.1, 50.8, 63.5, 76.2 , and $88.9 \mathrm{~mm}$ from the bottom exchanger. The two horizontal temperature arrays, placed at heights of 50.8 and $76.2 \mathrm{~mm}$, each consisted of four probes located 12.7, 25.4, 38.1, and $50.8 \mathrm{~mm}$ form the side wall (Fig. 2.5). 


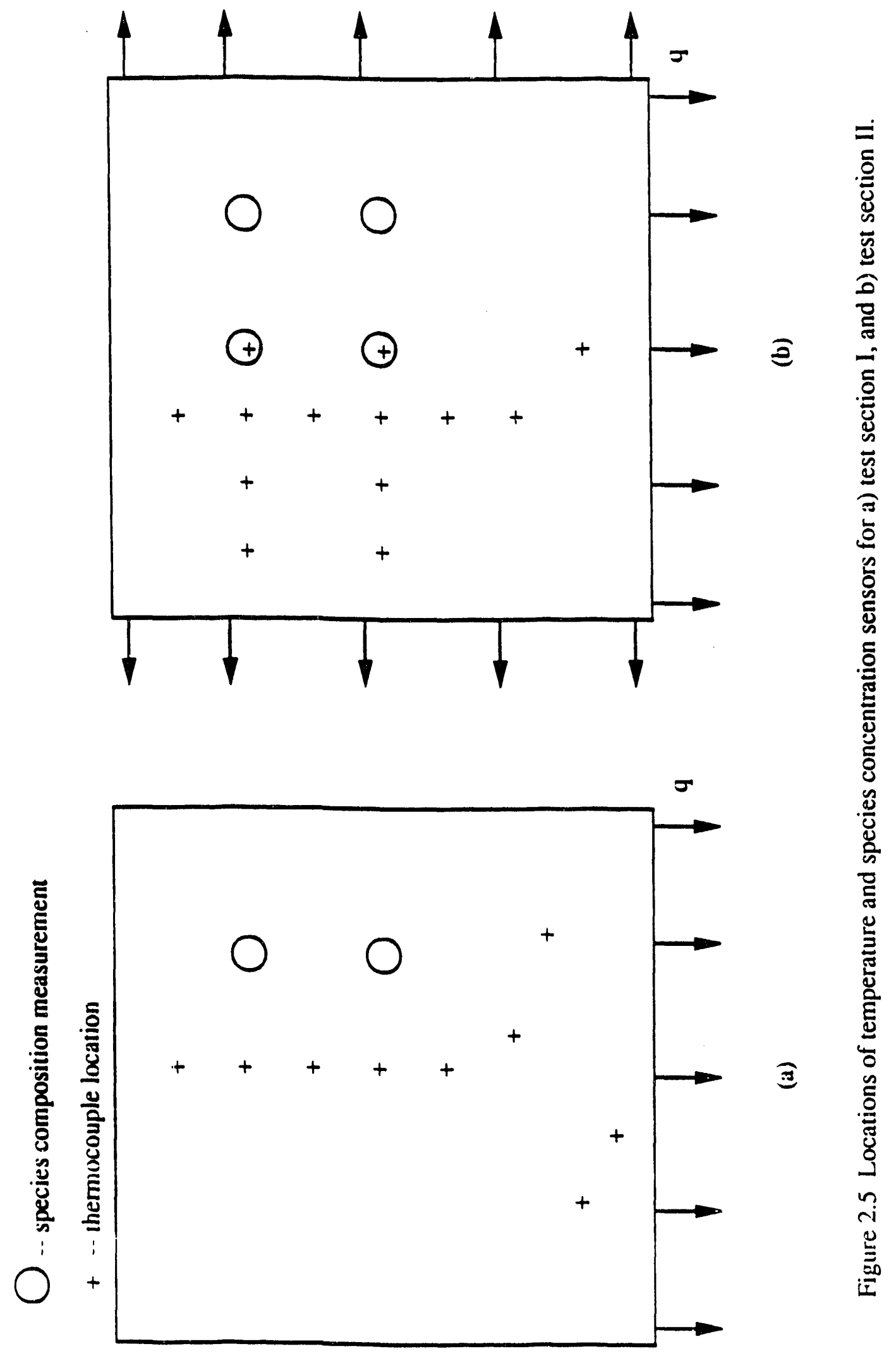


Thermocouples placed in the heat exchangers were used to measure cold plate temperatures. The thermocouple beads, three to four for each heat exchanger, were inserted through the back of an exchanger to within $2 \mathrm{~mm}$ of the chilled surface. Temperatures were recorded every fifteen seconds with a 3054 Hewlett-Packard data acquisition unit, and software compensation was used to process the voltages produced by the thermocouples.

\subsubsection{Species Concentration Measurement}

Species concentration measurements were taken every fifteen minutes for each experiment. In the UDS case (test section I), measurements were taken at distances of 50.8 and $76.2 \mathrm{~mm}$ above the heat exchanger surface. Horizontal concentration gradients were assumed to be negligible. When chilling from the two side and bottom walls (test section II), four measurements were made at elevations of 50.8 and $76.2 \mathrm{~mm}$ from the bottom heat exchanger and at horizontal distances of 25.4 and $50.8 \mathrm{~mm}$ from the side heat exchanger (Fig. 2.5). Actual sampling locations were estimated to be within $\pm 2.5 \mathrm{~mm}$ of the prescribed locations.

Measurements were taken by inserting a 20 gauge ( $1.27 \mathrm{~mm}$ diameter) stainless steel hypodermic needle into the melt and drawing out a small amount of fluid from the prescribed location. The needle was inserted into the melt through a brass, self-sealing temperature test plug supplied by Omega Engineering, Inc. Once drawn, the composition of the fluid was determined using a refractometer supplied by KernCo Instruments (El Paso, Texas). Operating on the optical principle that the refractive index of a solution is a function of its composition, the refractometer measures $\mathrm{NH}_{4} \mathrm{Cl}$ mass fraction to within \pm $0.1 \mathrm{wt} \%$. The overall accuracy of the species composition measurement method was estimated to be $\pm 0.5 \mathrm{wt} \%$. 


\section{CHAPTER 3 - MATHEMATICAL MODEL}

\subsection{Static Continuum Model}

In developing the continuum equations describing fluid motion in the phase change of a binary component system, the following assumptions were made to reduce the full set of Navier-Stokes equations: 1) negligible turbulence in the flow; 2) a Newtonian fluid; 3) local thermodynamic equilibrium; 4) constant properties, including the density of both the solid and liquid; 5) applicability of the Soussinesq approximation; 6) isotropic permeability; and 7) negligible species diffusion in the solid phase.

Mathematical modelling of a multik lase mixture, as found in the mushy region of a solidifying binary alloy, must account for the physical interaction occurring between phases contained within a particular control volume. The continuum model, originally derived by Bennon and Incropera (1987a) wind modified by Prescott et al. (1991), accounts for llow and transport conditions in the liquid, solid, and mushy regions by adopting principles of classic mixture theor'. The mixture thenry considers th: microscopic contributions and interactions of each phase on a macroscopic scale thus allowing the development of a continuum set of equations which can be solved with well established numerical computation methods.

Considering a control volume in the mushy region of a simple binary alloy, a dependent scalar variable is determined by the value of that variable found both in the solid and liquid phases. For example, thu density of a particular control volume depends on the weighted contributions of the liquid density and solid density according to

$$
\rho=g_{s} \rho_{s}+g_{l} \rho_{l}
$$


The values of velocity, energy, and species are similarly weighted in a two-phase control volume:

$$
\begin{gathered}
V=f_{s} V_{s}+f_{l} V_{l} \\
h=f_{s} h_{s}+f_{l} h_{l} \\
f^{\alpha}=f_{s} f_{s}^{\alpha}+f_{l} f_{l}^{\alpha}
\end{gathered}
$$

Equations (3.1)-(3.4) describe conditions in the all liquid, all solid, and mushy regions of a cast, thereby enabling the development of a single set of equations characterizing the entire solidifying domain.

The general conservation equation describing behavior of an arbitrary, dependent scalar variable, $\phi_{k}$, associated with phase $k$, in a multiphase region is given as (Bennon and Incropera, 1987a):

$$
\frac{\partial}{\partial t}\left(\bar{\rho}_{k} \phi_{k}\right)+\nabla \cdot\left(p_{k} V_{k} \phi_{k}\right)=-\nabla \cdot\left(g_{k} J_{k}\right)+g_{k} S_{k}
$$

Equation (3.5) balances the storage and advection of the variable $\phi_{\mathrm{k}}$ with its diffusion and production. Therefore, utilizing the continuum theory of mixtures, the complete set of continuum equations governing the conservation of mass, momentum, energy, and species in a solidifying binary alloy can be derived (Bennon and Incropera, 1987a and Prescott et al., 1991):

$$
\begin{gathered}
\frac{\partial}{\partial t}(\rho)+\nabla \cdot(\rho \mathbf{V})=0 \\
\frac{\partial}{\partial t}(\rho \mathrm{u})+\nabla \cdot(\rho \mathbf{V u})=\nabla \cdot\left(\mu_{l} \frac{\rho}{\rho_{l}} \nabla \mathrm{u}\right) \\
-\frac{\mu_{l} \rho}{\mathrm{K}_{\mathrm{x}} \rho_{l}}\left(\mathrm{u}-\mathrm{u}_{\mathrm{s}}\right)-\frac{\partial \mathrm{P}}{\partial \mathrm{x}}+\rho_{l} \mathrm{~g}_{\mathrm{x}}\left[\beta_{\mathrm{T}}\left(\mathrm{T}-\mathrm{T} \partial+\beta_{\mathrm{s}}\left(\mathrm{f}_{l}^{\alpha}-\mathrm{f}_{l, \mathrm{e}}^{\alpha}\right)\right]+\frac{\mathrm{D}}{\mathrm{D} t}\left(\bar{\rho}_{\mathrm{s}} \mathrm{u}_{\mathrm{s}}\right)\right. \\
\frac{\partial}{\partial \mathrm{t}}(\rho \mathrm{v})+\nabla \cdot(\rho \mathrm{Vv})=\nabla \cdot\left(\mu_{l} \frac{\rho}{\rho_{l}} \nabla \mathrm{v}\right) \\
-\frac{\mu_{l} \rho}{K_{\mathrm{y}} \rho_{l}}\left(\mathrm{v}-\mathrm{v}_{\mathrm{s}}\right)-\frac{\partial \mathrm{P}}{\partial \mathrm{y}}+\rho_{l} \mathrm{~g}_{\mathrm{y}}\left[\beta_{\mathrm{T}}\left(\mathrm{T}-\mathrm{T} \partial+\beta_{\mathrm{s}}\left(\mathrm{f}_{l}^{\alpha}-\mathrm{f}_{l, \mathrm{e}}^{\alpha}\right)\right]+\frac{\mathrm{D}}{\mathrm{Dt}}\left(\bar{\rho}_{\mathrm{s}} \mathrm{v}_{\mathrm{s}}\right)\right.
\end{gathered}
$$




$$
\begin{gathered}
\frac{\partial}{\partial t}(\rho h)+\nabla \cdot(\rho \mathbf{V h})= \\
\nabla \cdot\left(\frac{k}{c_{s}} \nabla h\right)+\nabla \cdot\left[\frac{k}{c_{s}} \nabla\left(h_{s}-h\right)\right]-\nabla \cdot\left[\rho f_{s}\left(h_{l}-h_{s}\right)\left(V-V_{s}\right)\right] \\
\frac{\partial}{\partial t}\left(\rho f^{\alpha}\right)+\nabla \cdot\left(\rho V f^{\alpha}\right)= \\
\nabla \cdot\left(\rho D \nabla f^{\alpha}\right)+\nabla \cdot\left[\rho D \nabla\left(f_{l}^{\alpha}-f^{\alpha}\right)\right]-\nabla \cdot\left[\rho f_{s}\left(f_{l}^{\alpha}-f_{s}^{\alpha}\right)\left(V-V_{s}\right)\right]
\end{gathered}
$$

The above set of equations describe solidification under an inertial reference frame or one that does not change with time. The second term on the right-hand side of the momentum equations (3.7-3.8) represents D'Arcy damping, where, under the assumption of slow, steady fluid motion, D'Arcy's law is employed to treat the flow through the porous mushy region. Buoyancy is accounted for in the momentum equation through the fourth terms on the right-hand side. The change of density with respect to both solutal concentration and temperature contribute to the overall buoyancy force. Changes in the direction of the gravity vector which accompany mold rotation is reflected in the $x$ and $y$ components of the gravitational acceleration associated with the buoyancy terms. The final term in the momentum equations, a solid acceleration term, accounts for movement of the solid region relative to the reference frame. This term is zero for the current solidification study.

Although columnar in nature, the crystalline mushy region is assumed to have an isotropic permeability governed by the following Kozeny-Carman type relation (Asai and Muchi, 1978):

$$
\mathrm{K}=\mathrm{K}_{0}\left[\frac{\mathrm{gl}_{l}^{3}}{\left(1-\mathrm{g}_{l}\right)^{2}}\right]
$$

Although not entirely accurate, the assumption of isotropic permeability qualitatively predicts flow and the formation of channels within the mushy region (Neilson and Incropera, 1991). The constant $\mathrm{K}_{0}$, which depends on the morphology of the mushy 
region, is given a value of $5.56 \times 10^{-12} \mathrm{~m}^{2}$ corresponding to a dendrite arm spacing of 32 $\mu \mathrm{m}$ (Neilson and Incropera, 1992a).

The enthalpy of phase $k$ ( $k$ is solid or liquid) is defined as

$$
h_{k}=\int_{0}^{T} c_{k} d T+h_{k}^{0}
$$

and similarly, the thermal conductivity and the mass diffusion coefficient are expressed as

$$
k=g_{s} k_{s}+g_{l} k_{l}
$$

and

$$
D=f_{l} D_{l}^{\alpha}
$$

The solid mass fraction and the species composition of the liquid $\left(f_{1}^{\alpha}\right)$ in a particular control volume are thermodynamically dependent on the temperature and overall composition (solid and liquid phase) of the control volume. Under the assumption of thermodynamic equilibrium, a linearized form of the equilibrium phase diagram determines solid mass fraction and $f_{1}^{\alpha}$ for the model equations such that

$$
\begin{gathered}
\mathrm{f}_{\mathrm{s}}=\frac{1}{1-\mathrm{k}_{\mathrm{p}}}\left[\frac{\mathrm{T}-\mathrm{T}_{\mathrm{liq}}}{\mathrm{T}-\mathrm{T}_{\mathrm{m}}}\right] \\
\mathrm{f}_{l}^{\alpha}=\left[\frac{1}{1+\mathrm{f}_{\mathrm{s}}\left(\mathrm{k}_{\mathrm{p}}-1\right)}\right] \mathrm{f}^{\alpha}
\end{gathered}
$$

where $T_{\text {liq }}$ is the liquidus temperature corresponding to $f^{\alpha}, T_{m}$ is the fusion temperature as $f^{\alpha}$ goes to zero, and $k_{p}$ is the ratio of the slopes of the liquidus and solidus lines (known as the equilibrium partition ration).

There are solidification phenomena which the above formulation does not address. The assumption of thermodynamic equilibrium during phase change is a good first approximation which adequately predicts general macroscopic behavior such as 
growth front progression and the formation of channels; however, non-equilibrium effects such as undercooling and recalescence are present during solidification and may be primary sources of error in the numerical model. Although the present model does not address non-equilibrium effects, extensions to the model which do address these effects have been made (Prescott and Incropera, 1992b). Another potential source of error in the present model, particularly when applied to the solidification of metal alloys, is the assumption of a continuous solid crystalline structure. Observations of slurry regions in solidifying $\mathrm{NH}_{4} \mathrm{Cl}-\mathrm{H}_{2} \mathrm{O}$ systems (Murakami and Okamoto, 1984) contradict this assumption. Here too, efforts have been made to improve the existing model by accounting for free floating crystals in the melt (Prescott and Incropera, 1992b). However, this improved model, known as the slurry model, assumes free floating crystals to be broken from the edge of the liquidus region by vigorous convection motion in the melt. An improvement in simulating the solidification of a metal alloy from a side wall, the slurry model has not been applied to the present study where the slurry region is small and originates from deep within the mushy region instead of along the liquidus interface.

\subsection{Inertial Effects}

The above set of equations is derived for an inertial coordinate system or one that does not move with time. To account for an oscillatory motion of the moving coordinate system in space, fluid particle acceleration within the solution domain must be related to an inertial frame of reference. Following the development of Kaplan (1976), the absolute acceleration of a particle described in a non-inertial frame of reference is given as

$$
\mathbf{a}=\mathbf{a}_{0}+\ddot{\mathbf{r}}+2 \Omega \times \dot{\mathbf{r}}+\frac{\partial \Omega}{\partial \mathrm{t}} \times \mathbf{r}+\Omega \times(\Omega \times \mathbf{r})
$$

where $r$ is the position vector of a particle relative to the moving frame, $\Omega$ is the angular velocity vector of the moving frame, $a_{0}$ is the acceleration of the origin of the moving 
frame, $\ddot{\mathbf{r}}$ is the apparent acceleration of a particle in the moving frame, $2 \Omega \times \dot{r}$ is the Coriolis acceleration, $(\partial \Omega / \partial t) \times r$ is the angular acceleration, and $\Omega \times(\Omega \times r)$ is the centripetal acceleration.

The present problem considers a two-dimensional rectangular box which rotates around the $\mathrm{z}$ axis such that

$$
\boldsymbol{\Omega}=\boldsymbol{\Omega} \mathbf{k} .
$$

Including the effect of the inertial terms in the conservation of momentum equations, (3.7-3.8), the $x$ and $y$ components of momentum modeling the phase chase of a binary solution undergoing rotation around the $\mathrm{z}$ axis become

$$
\begin{gathered}
\frac{\partial}{\partial t}(\rho u)+\nabla \cdot\left(\rho V_{u}\right)= \\
\nabla \cdot\left(\mu_{l} \frac{\rho}{\rho_{l}} \nabla u\right)-\frac{\mu_{l} \rho}{K_{x} \rho_{l}}\left(u-u_{s}\right)-\frac{\partial P}{\partial x}+\rho_{l g}\left[\beta_{\mathrm{T}}\left(T-T_{\partial}\right)+\beta_{s}\left(f_{l}^{\alpha}-f_{l, e}^{\alpha}\right)\right] \\
+2 \rho v \Omega+\rho\left(x-X_{0}\right) \Omega^{2}+\rho\left(y-Y_{0}\right) \frac{\partial \Omega}{\partial t} \\
\frac{\partial}{\partial t}(\rho v)+\nabla \cdot\left(\rho V_{v}\right)= \\
\nabla \cdot\left(\mu_{l} \frac{\rho}{\rho_{l}} \nabla v\right)-\frac{\mu_{l} \rho}{K_{y} \rho_{l}}\left(v-v_{s}\right)-\frac{\partial P}{\partial y}+\rho_{l} g_{y}\left[\beta_{T}(T-T)+\beta_{s}\left(f_{l}^{\alpha}-f_{l, l}^{\alpha}\right)\right] \\
-2 \rho u \Omega+\rho\left(y-Y_{0}\right) \Omega^{2}-\rho\left(x-X_{0}\right) \frac{\partial \Omega}{\partial t}
\end{gathered}
$$

where $X_{0}$ and $Y_{0}$ are the $x$ and $y$ displacements from the center of rotation to the origin of the moving frame. The final three terms on the right-hand side of the momentum equations are Coriolis, centripetal, and angular accelerations, respectively.

For the particular study of a cast experiencing a sinusoidal rocking, oscillation of the mold is described as follows:

$$
\begin{gathered}
\theta=-\theta_{0} \cos (\omega t) \\
\Omega=\dot{\theta}=\theta_{0} \omega \sin (\omega t)
\end{gathered}
$$




$$
\dot{\Omega}=\ddot{\theta}=\theta_{0} \omega^{2} \cos (\omega t)
$$

where $\theta$ is the angular displacement, $\theta_{0}$ is the amplitude, and $\omega$ is the rocking frequency.

Each inertial term on the right-hand side of equations (3.19) and (3.20) have been normalized and compared to the buoyancy term, the primary source of convection. Each inertial term varies from zero to its maximum value twice in a particular cycle, and each term's maximum value is comparable to the value of the buoyancy term. Therefore, all inertial terms were kept in the momentum equations of the rocking model.

\subsection{Solution Procedure}

Numerical implementation of the continuum equations follows a finite-difference scheme, originally developed by Bennon and Incropera (1987b, 1988), which is based on the SIMPLER algorithm (Patankar, 1980). In the SIMPLER methodology, a parabolic set of equations cast in a generalized form are integrated over space and marched in time for each control volume yielding a solvable set of algebraic : sons. Time marches at one second intervals for each simulation, and the domain is discretized with an orthogonal Cartesian grid weighted near the boundaries. The mesh of $102 \times 66$ nodes was determined from grid independence studies for the UDS of $\mathrm{NH}_{4} \mathrm{Cl}-\mathrm{H}_{2} \mathrm{O}$. Although never

truly grid independent, the prescribed mesh has been determined adequate for capturing important macroscopic effects (Neilson and Incropera, 1991). Thermophysical properties of $\mathrm{NH}_{4} \mathrm{Cl}-\mathrm{H}_{2} \mathrm{O}$ used for the current study were taken from the literature (Neilson and Incropera, 1991). Finally, in the present model, the power law, as opposed to an upwinding scheme, is used in determining the convection/diffusion influence coefficients. 


\section{CHAPTER 4 - EXPERIMENTAL RESULTS OF STATIC SOLIDIFICATION}

\subsection{Introduction}

In order to provide a baseline to assess the effects of rocking on solidification, a detailed set of experiments have been performed for static solidification of an aqueous $27 \% \mathrm{NH}_{4} \mathrm{Cl}$ solution. Although many investigators have previously considered static castings (see Chapter 1), the test section of this study was well suited to obtaining unique and insightful observations. To prepare an experiment, a well mixed solution was allowed to equilibrate thermally and come to rest in the test section. One minute after temperature measurements were initiated, coolant at $-30^{\circ} \mathrm{C}$ was impulsively applied to the heat exchangers, thereby rapidly dropping the cold wall surface temperatures. Many solidification experiments have been performed and the findings are presented in the following sections:

\subsection{Unidirectional Solidification}

\subsubsection{Qualitative Results}

Within 15 seconds of routing coolant to the bottom heat exchanger of test section I, dendritic crystals began to form on the cold horizontal surface (Fig. 4.1). Within another 20 seconds, the cold surface was completely covered with a crystalline mush about $4 \mathrm{~mm}$ thick. The crystal structure was characteristically dendritic and tightly packed, and the large rate of nascent crystal growth suggested the existence of substantial fluid undercooling. 

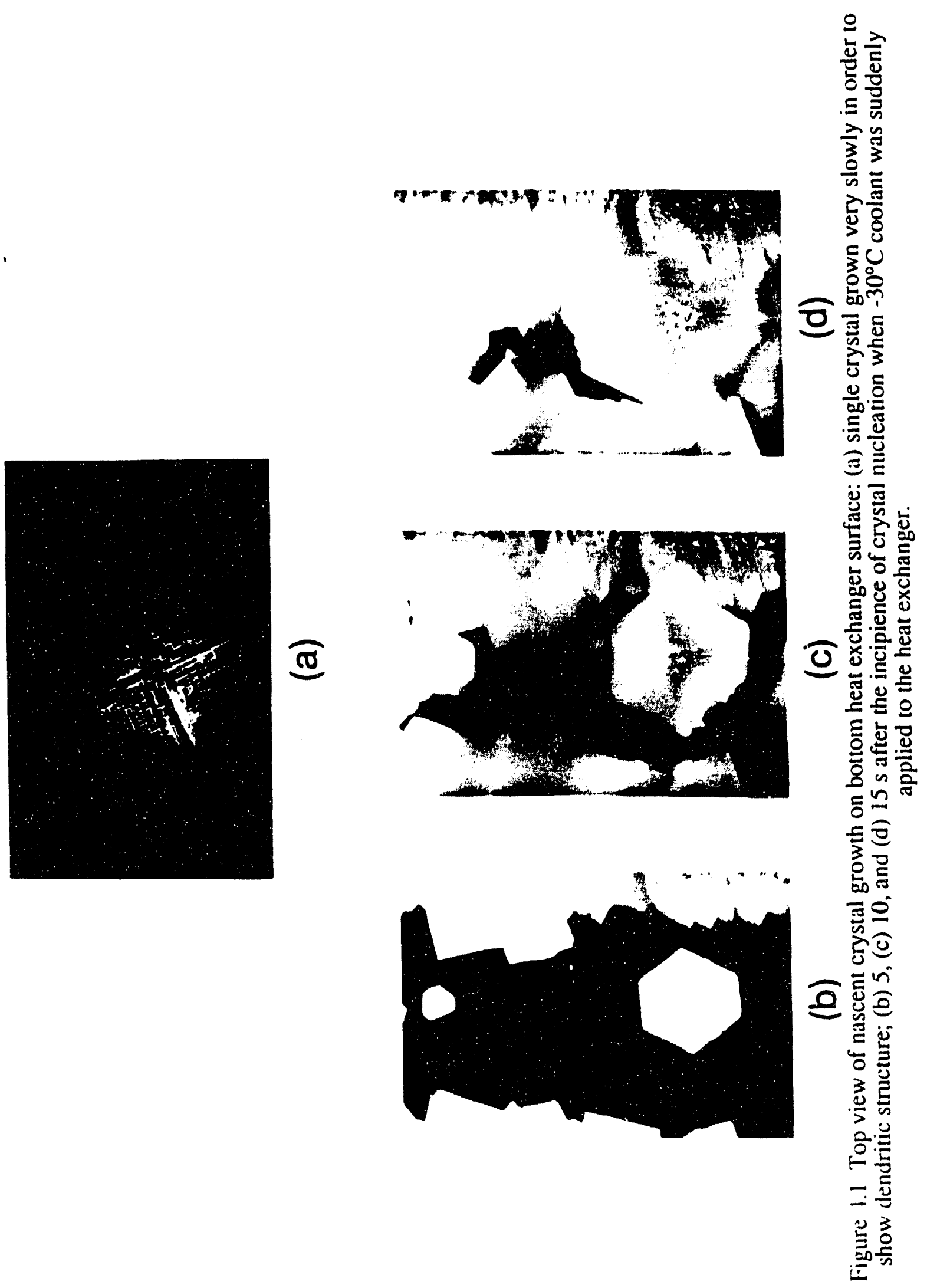
As crystals grew, they rejected lighter, water-rich fluid which immediately promoted salt fingering from the liquidus interface (Fig. 4.2). This double-diffusive instability yielded ascending water-rich fingers of fluid adjacent to descending salt-rich fingers (Turner, 1974). As the first water-rich fingers rose, they increased in size and developed a mushroom, or top-hat, like appearance, which is similar to the behavior observed in rising thermals (Gebhart et al., 1988). Owing to the relatively high Lewis number of $\mathrm{NH}_{4} \mathrm{Cl}-\mathrm{H}_{2} \mathrm{O}$, the first salt fingers retained their compositional identity and were clearly visible as they rose to the top of the test section.

The fingers strengthened and remained organized until, at approximately two minutes, the most vigorous fingers began to eject small crystals into the bulk liquid. It is not clear whether these crystals were small equiaxed dendrites that nucleated in the undercooled interdendritic fluid, whether they were dendrite tips that fractured due to the increasingly vigorous salt fingering, or whether they were crystals generated by a combination of both effects. In any case, at approximately five minutes, from 150 to 200 robust salt fingers were spewing discrete crystals into the melt just above the liquidus interface (Fig. 4.3a). This slurry zone appeared to be approximately $5 \mathrm{~mm}$ thick. As small crystals were ejected by the salt fingers, some continued to ascend into the bulk liquid where they melted; however, most crystals migrated from the ascending water-rich fingers to adjacent, descending fingers. As a particular crystal descended, it rapidly grew in size, such that its dendritic structure could be seen by the naked eye. It is this increased size that prevented the crystals from penetrating the porous mushy region, causing them instead to fall onto the dendritic tips of the advancing liquidus front.

Crystals rejected from a UDS mushy region have been observed before (Neilson, 1991). Those observations suggested that the UDS slurry was much more vigorous for weaker $\mathrm{NH}_{4} \mathrm{Cl}$ concentration mixtures. Moreover, from the trends of the present study, crystals seem to become more prevalent with decreasing initial melt temperature, and 

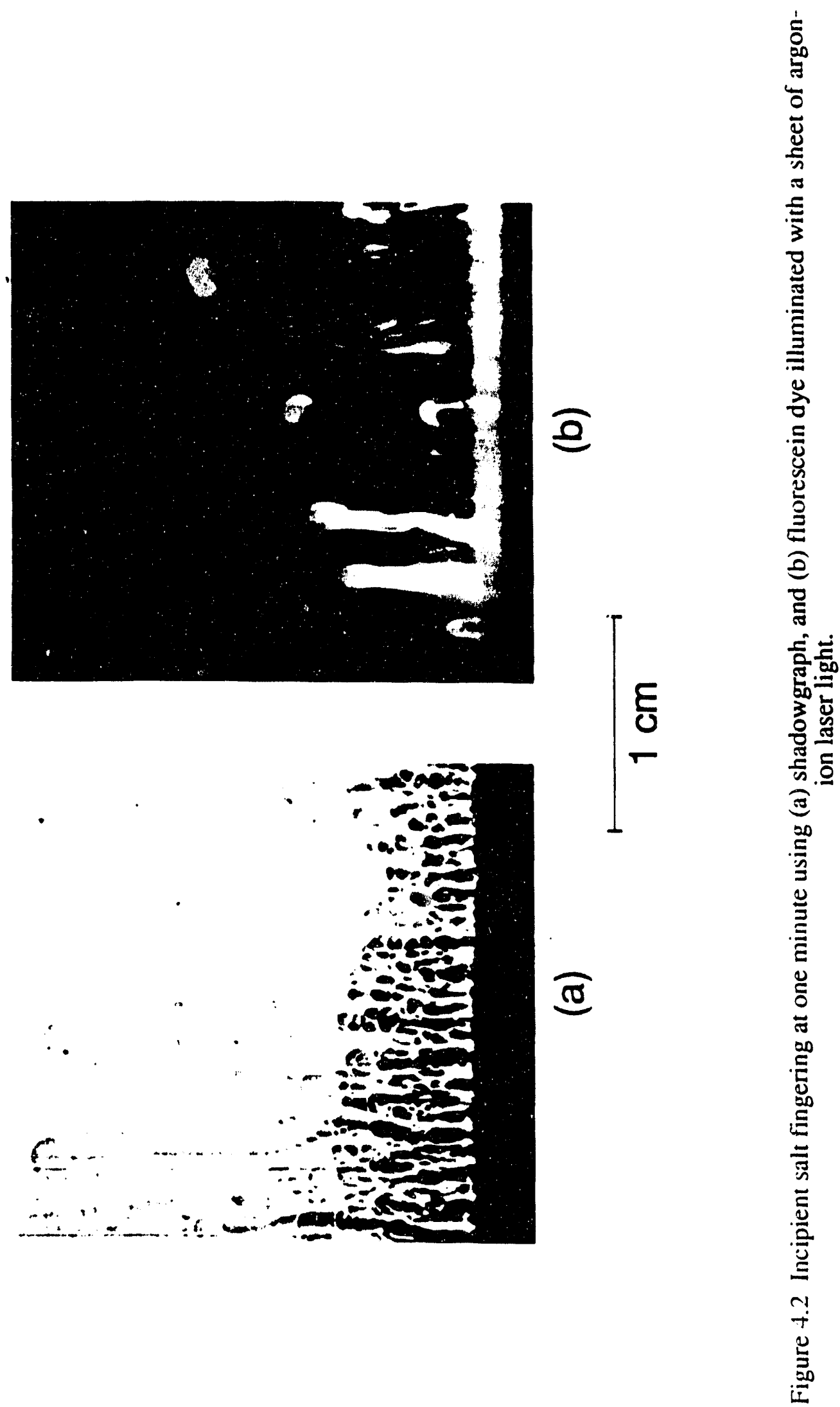

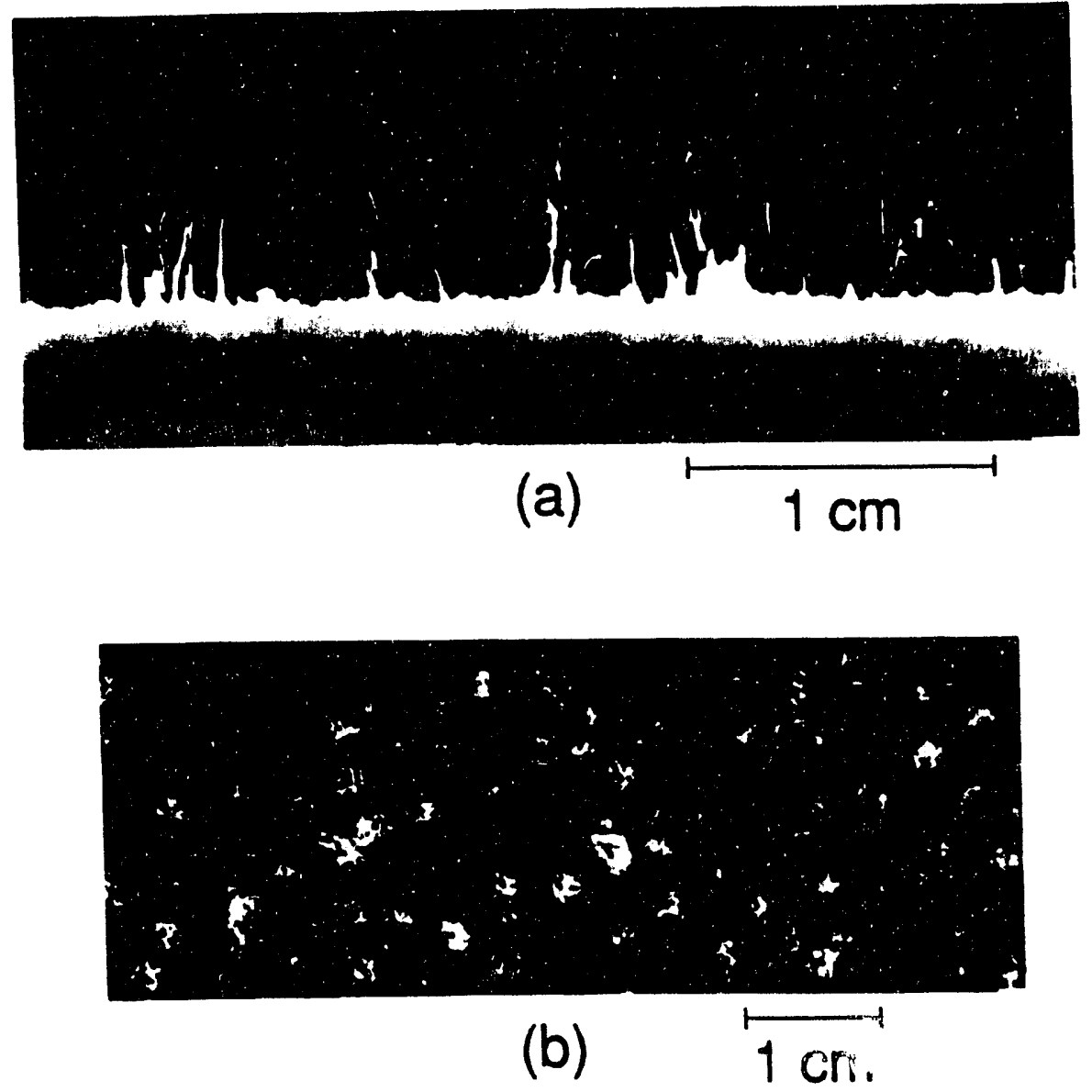

Figure 4.3 (a) Time exposure of slurry zone above advancing liquidus front during unidirectional solidification (UDS) at $300 \mathrm{~s}$ showing discrete fountain sites where crystals are ejected into the melt. (b) Overhead view of equiaxed mounds resulting from the slurry. 
hence decreasing thermal gradient. A low thermal gradient allows stronger salt fingering, which in turn, may lead to more fractured crystals ejected into the melt.

Eventually, rings of equiaxed crystals collected around the mouths of the most vigorous salt fingers, and the liquidus interface became strewn with hillocks that surrounded each robust finger (Fig. 4.3b). The subsequent growth of these equiaxed igs also rejected water-rich fluid, which seemed to augment development of the associated fingers. However, it is important to note that within the equiaxed rings, the fingers had not yet begun to melt away noticeable holes in the mushy region. Subsequently ( $t \approx 15 \mathrm{~min}$ ), slurry formation diminished, and the equiaxed hillocks were overgrown by the advancing liquidus front. Of the 150 to 200 small fingers which existed originally, only a handful were sufficiently strengthened to become the source of mature channels which formed in the mushy region.

Figure 4.4 shows the transition of numerous salt fingers to a much smaller number of plumes. In general, neighboring fingers and plumes competed for the supply of waterrich, interdendritic fluid, and only the strongest fingers survived to become sources of channels. After strong fingers established themselves, small holes began to form in the mushy region immediately below these fingers. The holes were initially located in the weaker crystalline structure between primary dendrites, and as the holes grew, the associated plumes issuing from the mush strengthened. Over time the holes grew to a diameter of about $1.5 \mathrm{~mm}$ and became pathways for cold, near eutectic fluid to issue from the mush and rise to the top of the test section. Characteristic of salt finger-type convection, particle tracking experiments revealed that fluid motion was strikingly vertical as strong fingers and plumes established themselves. In addition, large crystalline volcanos formed around the mouths of the robust channels (Fig. $4.5 \mathrm{c}$ and d).

Competition between neighboring finger sites to advect light, interdendritic fluid into the melt becomes evident very early in the experiments, as salt fingers develop and 

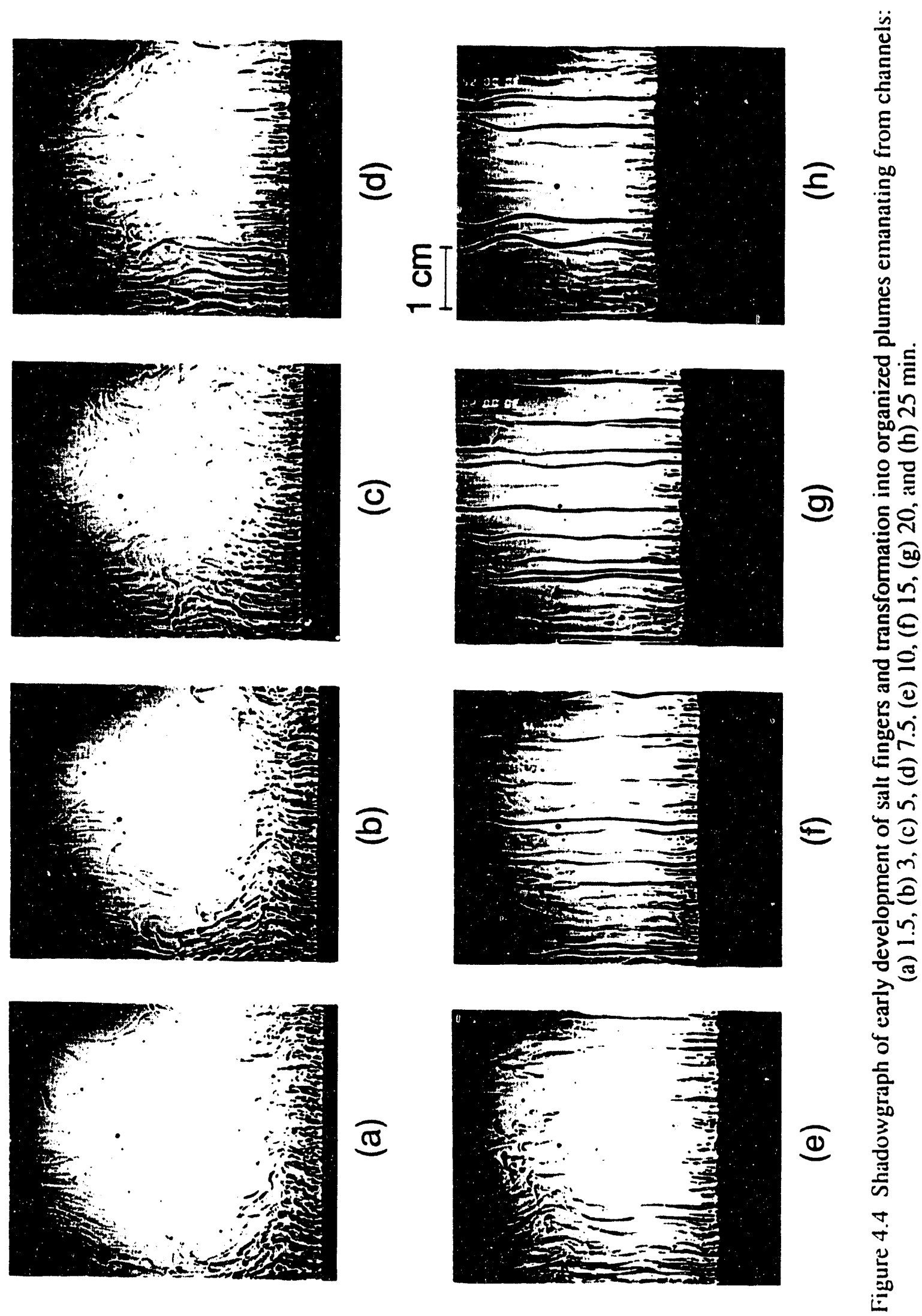

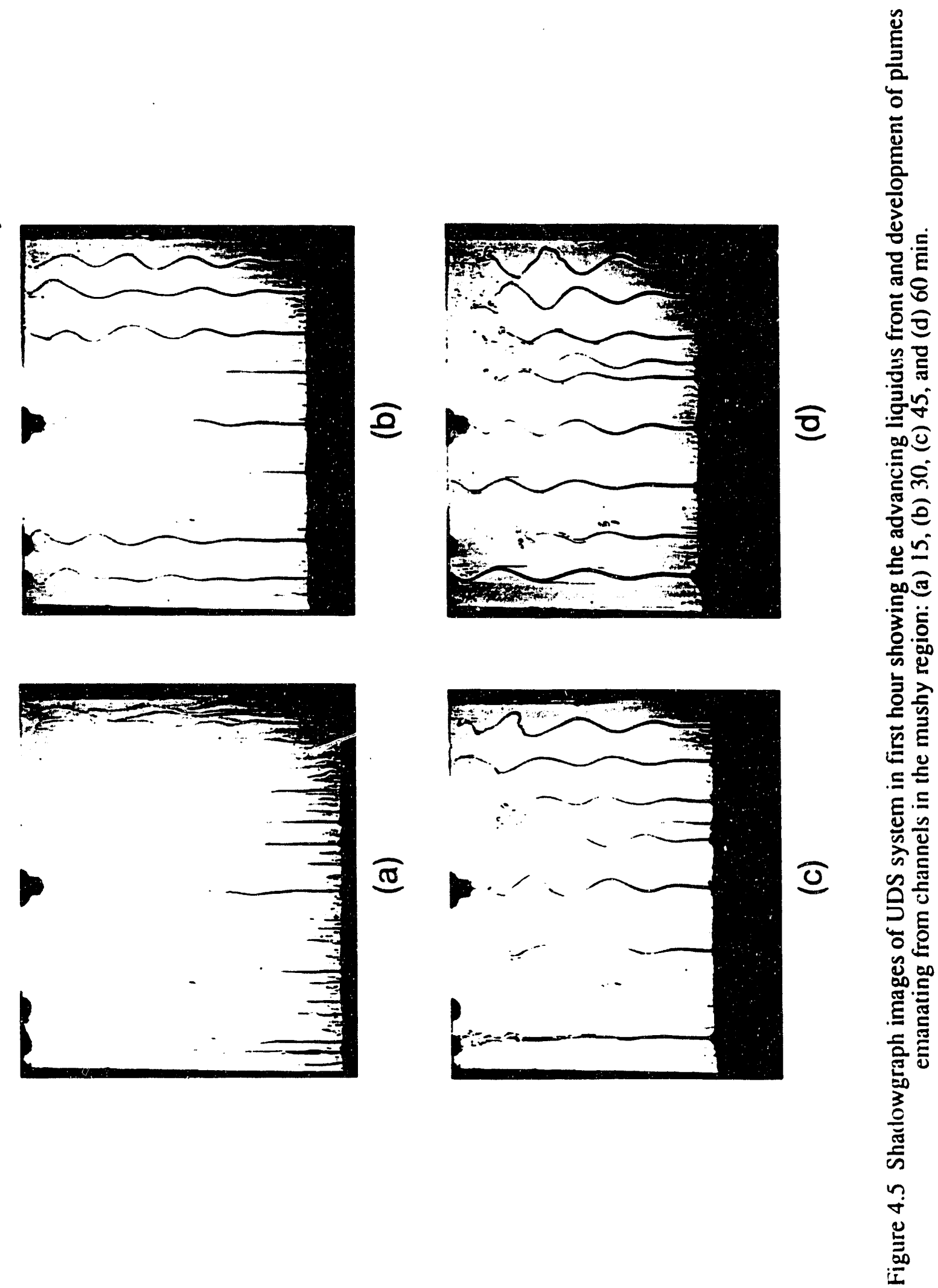
coalesce. Fingers evolve into a matrix of columns competing to transport the light interdendritic fluid to the top of the test section. However, this localized salt fingering phenomenon does not efficiently drain water-rich fluid from deep within the dendritic forest, and the lighter, nearly eutectic fluid needs an improved mechanism to be advected into the bulk liquid.

It has been demonstrated that a hole, artificially created in the mush, is not enough to form plumes and related channel development. Using a needle, Sample and Hellawell (1984) punctured a hole in the crystal structure of a mature mushy region and observed no sudden release of interdendritic fluid. However, when fluid was gently withdrawn from the liquidus interface using a hypodermic needle, a plume, and subsequently, a channel formed. Buoyant convection at the liquidus interface draws water-rich interdendritic fluid upward, thereby melting the crystal structure and forming a channel. Once a strong flow of fluid through a channel is started, it perpetuates itself, much like a siphon. Therefore, a relatively strong finger must be present before the concomitant formation of a channel.

The flow visuadization of this study provided some insight on how one finger may gain an advantage over its neighbors and initiate a channel. In the UDS arrangement, elevation appears to provide the necessary edge. Early in the process, the strongest fingers rose from elevated locations in the dendritic front. Indeed, the equiaxed mounds formed by the slurry zone tended to strengthen their accompanying plumes. During the dye injection experiments, the hypodermic needles acted as nucleation sites for crystals ahead of the advancing liquidus front, and hillocks formed at the needle locations. Although the dendrite front eventually overgrew the needle locations, channels subsequently formed at these locations, indicating that the early hillocks generated large localized salt fingers that eventually formed channels long after the hillocks themselves were overgrown. In the eariy experiments by Copley et al. (1970), it was determined that 
channels tended to form on the outside of concave liquidus fronts and at the center of convex fronts. More recently, simulating the UDS of a lead-tin alloy, Felicelli et al. (1991) concluded that channels tend to form at the leading part of the liquidus front. In summary, there is evidence to suggest that elevated locations on the liquidus front during early salt fingering become preferred sites for channel formation.

The foregoing condition of channel development may be rooted in one or both of the following effecis. First, there is a tendency for light, interdendritic fluid to be drawn toward high points along the crystalline front. Much like the rising thermal boundary layer on an inclined plate at low Rayleigh numbers, viscous forces cause the light waterrich fluid at a liquidus front to ascend along the sides of hillocks, thereby augmenting the associated salt finger. Second, and perhaps more importantly, the additional crystal surface area present in the hillocks acts to reject larger amounts of water-rich fluid than the surrounding mush. Therefore, by virtue of a larger volumetric rejection of light fluid, hillocks and mounds along the liquidus interface promote stronger fingers. Well established fingers will then draw interdendritic fluid from within the mush and develop concomitant channels.

Recognizing that elevated sites in the liquidus front lead to channel formation, a scheme was developed to produce freckles at pre-determined locations within the cast. Using short, vertical fins extending from the bottom cold wall, crystal growth around the elevated fins would produce hills along the liquidus, thereby nurturing channel growth at the fin sites. The growing channels suppressed channel formation in regions adjacent to the fins, suggesting that it may be possible to induce freckles in locations of the cast which are not critical to the strength or performance of the final product. For example, in a turbine blade, freckles could be placed in areas which are to be removed through postprocessing. The results of this work can be found in Appendix A. 
While plumes strengthened, salt fingering still persisted in regions between plumes. However, the fingers slowly weakened, and ultimately, the plumes rose primarily through a quiescent, solutally stratified melt. In the later stages of the experiment, all salt fingering was ultimately suppressed, and the plumes became the primary mechanism for convective transport of interdendritic fluid into the melt (Fig. 4.6). Manifestations of double-diffusive layering are provided by the horizontal diffusive interfaces which are barely visible.

A plume rising from a channel consisted of nearly eutectic fluid which, although quite cold, was driven by very strong solutal buoyancy forces. As the plume rose, it quickly gained energy through thermal diffusion from the surrounding warm meit, but owing to a relatively high Lewis number, the plume maintained its solutal identity. The additional thermal buoyancy augmented the plume's upward velocity and carried it robustly to the top of the test section. However, although always laminar, plume motion did become unstable.

The largest plume velocities were estimated to be approximately $10 \mathrm{~mm} / \mathrm{s}$, which corresponded to a Reynolds number, based on plume diameter, of approximately 10 . As the Reynolds number increased with increasing velocity, the plume began to develop an unstable oscillation. The oscillation appeared sinusoidal in nature and was initially confined to a two-dimensional vertical plane that bisected the axis of the channel from which the plume emanated. This plane of oscillation rotated very slowly around the channel axis and, with two-dimensional shadowgraphic imaging, created the illusion that the plume oscillation came and went with time. In other words, although strong plumes continuously oscillated, they sometimes appeared to be characterized exclusively by vertical motion when viewed on the shadowgraph imaging sheet. The wavelength of the oscillations was generally on the order of $14 \mathrm{~mm}$, and the oscillation waves propagated upward at approximately $7.5 \mathrm{~mm} / \mathrm{s}$. 

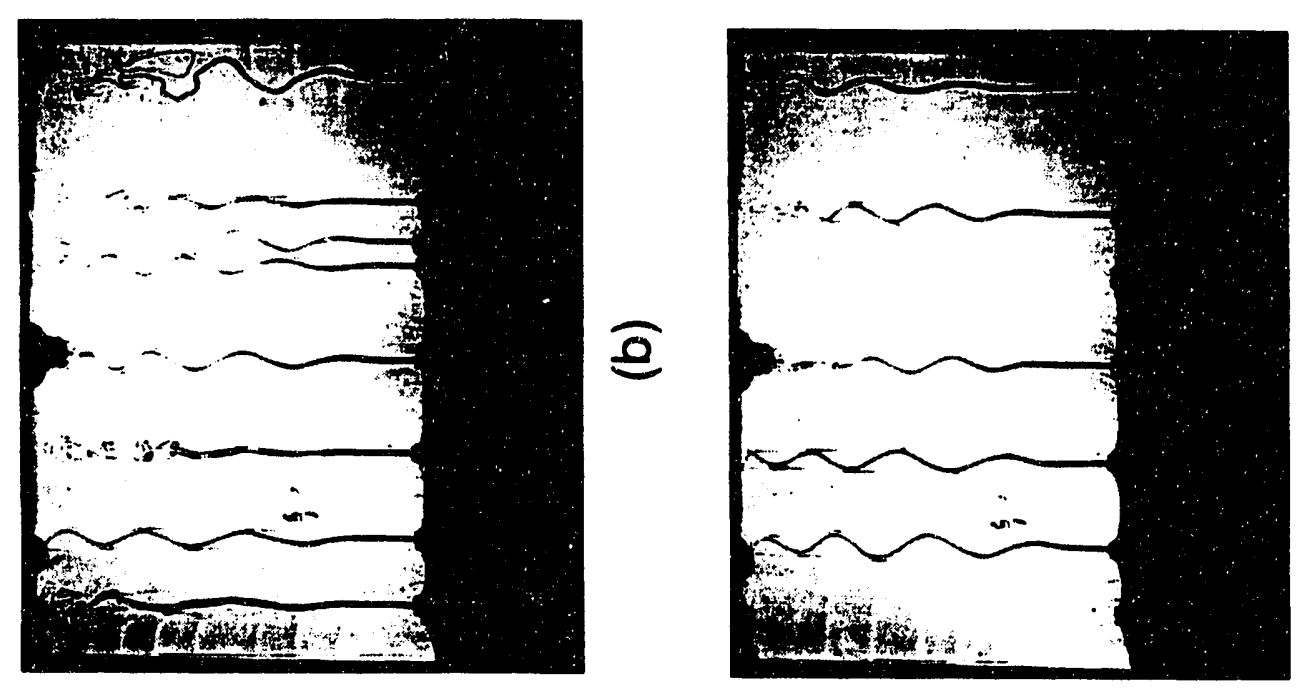

官.

호

을

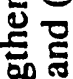

논

苟

흐

ํํำ

荧㓉

ชิำ

蓄

究

노ㄹㅗㅗ
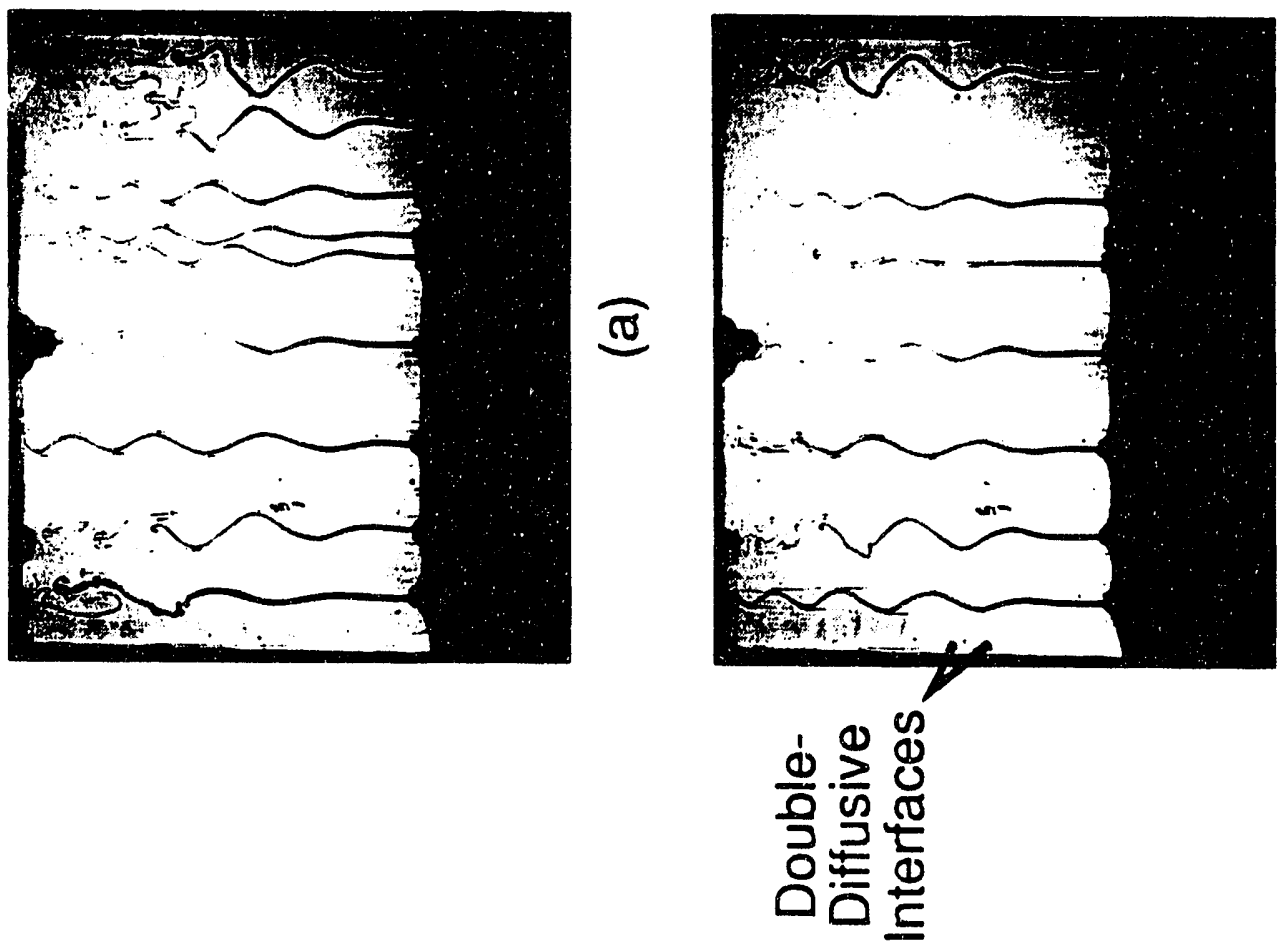

U

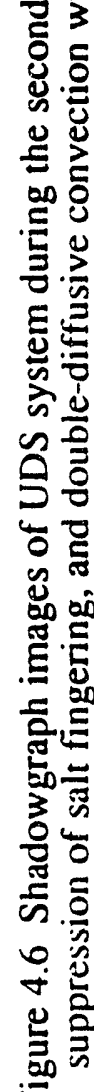


As a plume oscillated in its plane, fluid motion in the outer bends of the oscillation was retarded by viscous drag forces. However, as fluid continued to ascend from below the bend, it induced a bifurcation of fluid motion in the bend causing the plume to fold onto itself and diverting less buoyant fluid from above. If viewed from a position perpendicular to the plane of oscillation, this plume buckling would have a mushroom-shaped appearance. Figure 4.6b shows this plume buckling phenomenon from both a side view (farthest plume on the right) and an end view (third plume from the left).

As the Reynolds number increased, motion of the most vigorous plumes became helical in nature, and wisps of falling fluid appeared to be shed from the outer bends of the plumes (Fig. 4.7). These wisps were seen with both the shadowgraph and fluorescein dye (Fig. 4.8a) techniques. Although the wisps were most pronounced with helical plumes, they were also observed with the sinusoidal planar plumes. A progression of motion from sinusoidal, to helical, and subsequently to turbulence with increasing Reynolds number has been observed by Crow and Champagne (1971) for laminar, circular jets discharged into a quiescent fluid.

Once a channel is established, the associated plume draws very cold, nearly eutectic fluid from the lower portions of the mushy region. This column of water-rich fluid is warmed as it rises in the melt, while concurrently, salt-rich fluid adjacent to the plume is chilled. The chilled fluid then descends to the mouth of the channel, promoting crystal growth and forming the characteristic volcanos associated with mature channels.

The wisps of fluid associated with bends in an oscillating plume also result from the plume's thermal influence. Just as the bottom of the plume has a sheath of chilled, descending salt-rich fluid encircling it, upper sections of the plume similarly have a sheath of chilled descending fluid. However, when the plume begins to oscillate, this sheath cannot traverse the plume bends and the associated fluid detaches from the plume 


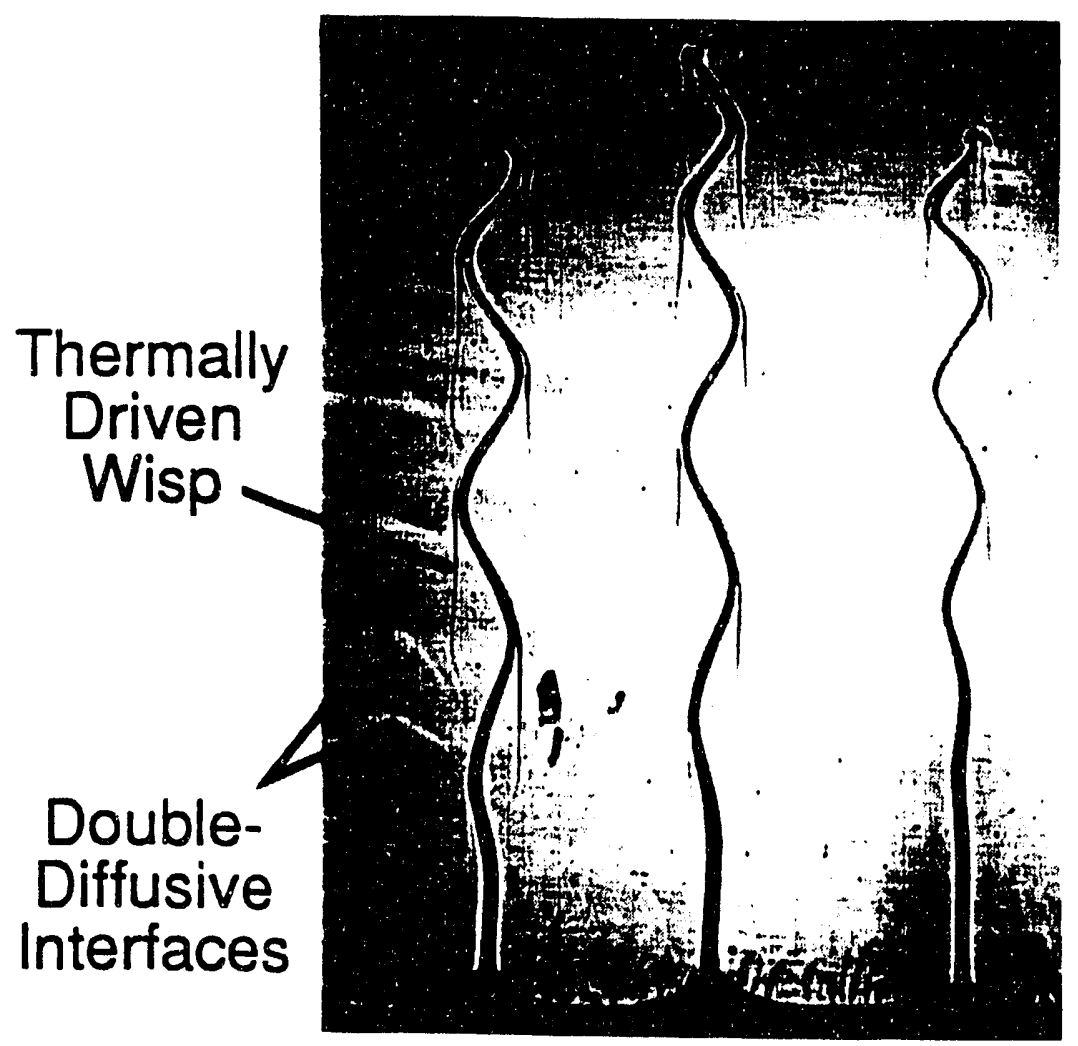

Figure 4.7 UDS shadowgraph image at 106 minutes of three helical plumes showing double-diffusive layering and cold 'wisps' of fluid falling from bends in the plume. Waves in the plume propagate upwards. 

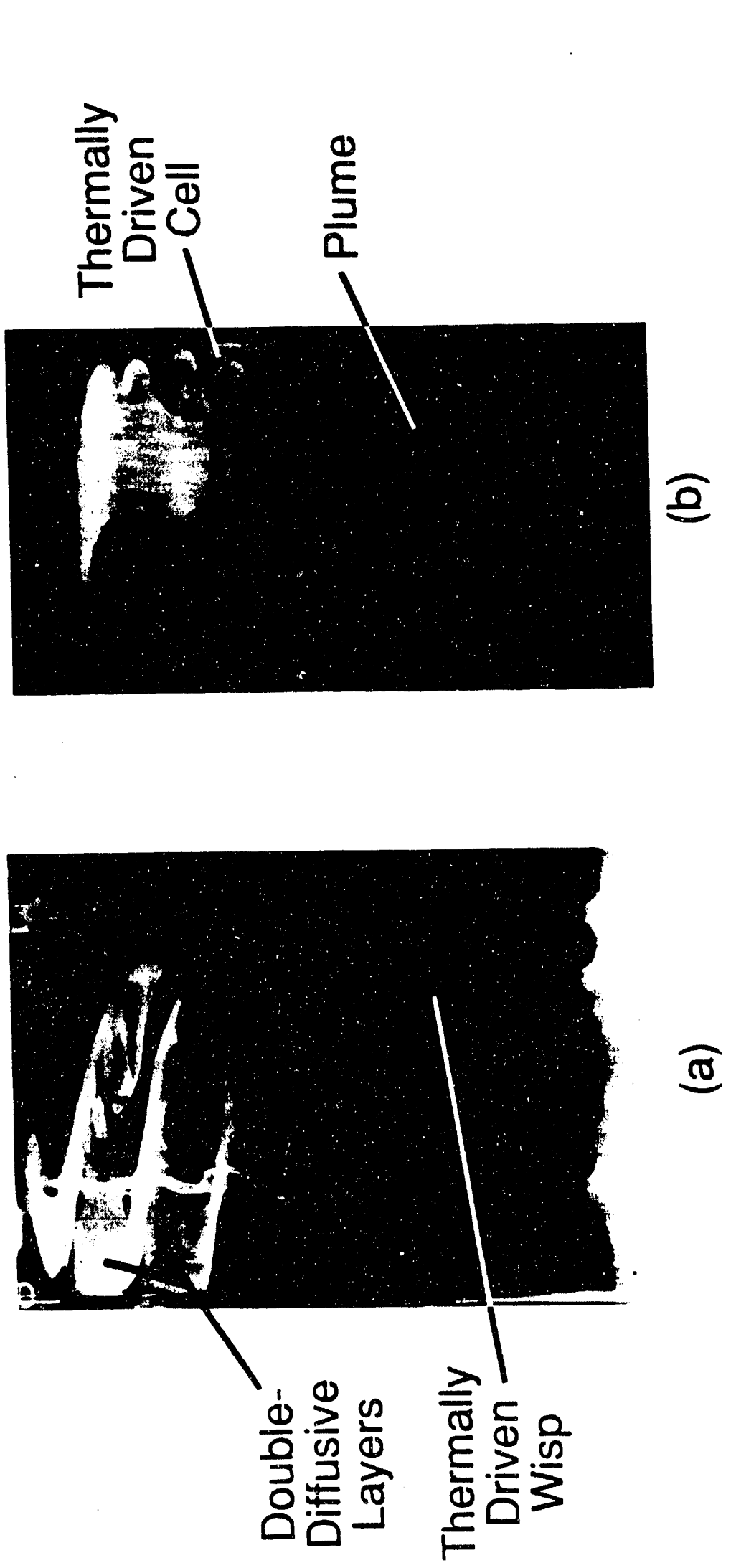

颉高

它究

을

कै

छํ.

을형

20

드ㄴㅡㅡ

ํㅣㄹ

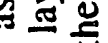

웡

3 赑

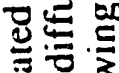

ن

品等

ㅎํㅇ.

을

㓉

政

竞

들

i

응

言苍吉

음

ত্

도

층몷

둥

.

晋远

응

总

$\infty$

氮

渠命 
and descends freely (Fig. 4.9). Owing to their depressed temperature, these wisps of fluid are visible with the shadowgraph technique (Fig. 4.7).

About one hour into the experiment, slight double-diffusive layers began to develop in the bulk liquid. The layers were initially most pronounced at the side walls of the test section, where cold rising plumes generated a horizontal thermal gradient. Using fluorescein dye injection to track a plume, Figures $4.8 \mathrm{a}$ and $4.8 \mathrm{~b}$ show the characteristic double-diffusive layering behavior which involved stacked, thermally driven convection cells separated by stable diffusive interfaces. In this case, the cells extend from the plume to the mold wall. Although they are strongest on the mold wall side of the plume, the cells, in effect, surround the cold plume, essentially forming a tower of stacked, rolling tori. Using particle tracking, Figure 4.10 shows the distinctly cellular fluid motion characteristic of the double-diffusive convection cells surrounding a meandering plur e. The cold plume thermally drives the cellular movement within each double-diffusive layer, and eventually, the double-diffusive layers extend across the entire width of the test section (Fig. 4.11).

As shown by Chen and Turner (1980), a solutally stratified fluid that is subject to a horizontal thermal gradient will develop distinct, double-diffusive convection cells. The cells are separated by very stable interfaces, and within a particular cell, fluid flow is driven by thermal buoyancy. The same mechanisms exist in the UDS system. During the first hour of solidification, the melt gradually stratified, as cold but lighter, nearly eutectic fluid was transported to the top of the cavity by the plumes. These cold plumes established a horizontal thermal gradient which induced the formation of double-diffusive cells. The cells began to form at the sidewalls of the cavity, where the ambient heat gain (hence, the thermal gradient) was strongest, and slowly propagated across the test section.

Figure 4.12 shows the crystalline structure from the top and side. In the static UDS cast, a number of distinct regions exist within the mushy zone where each primary 


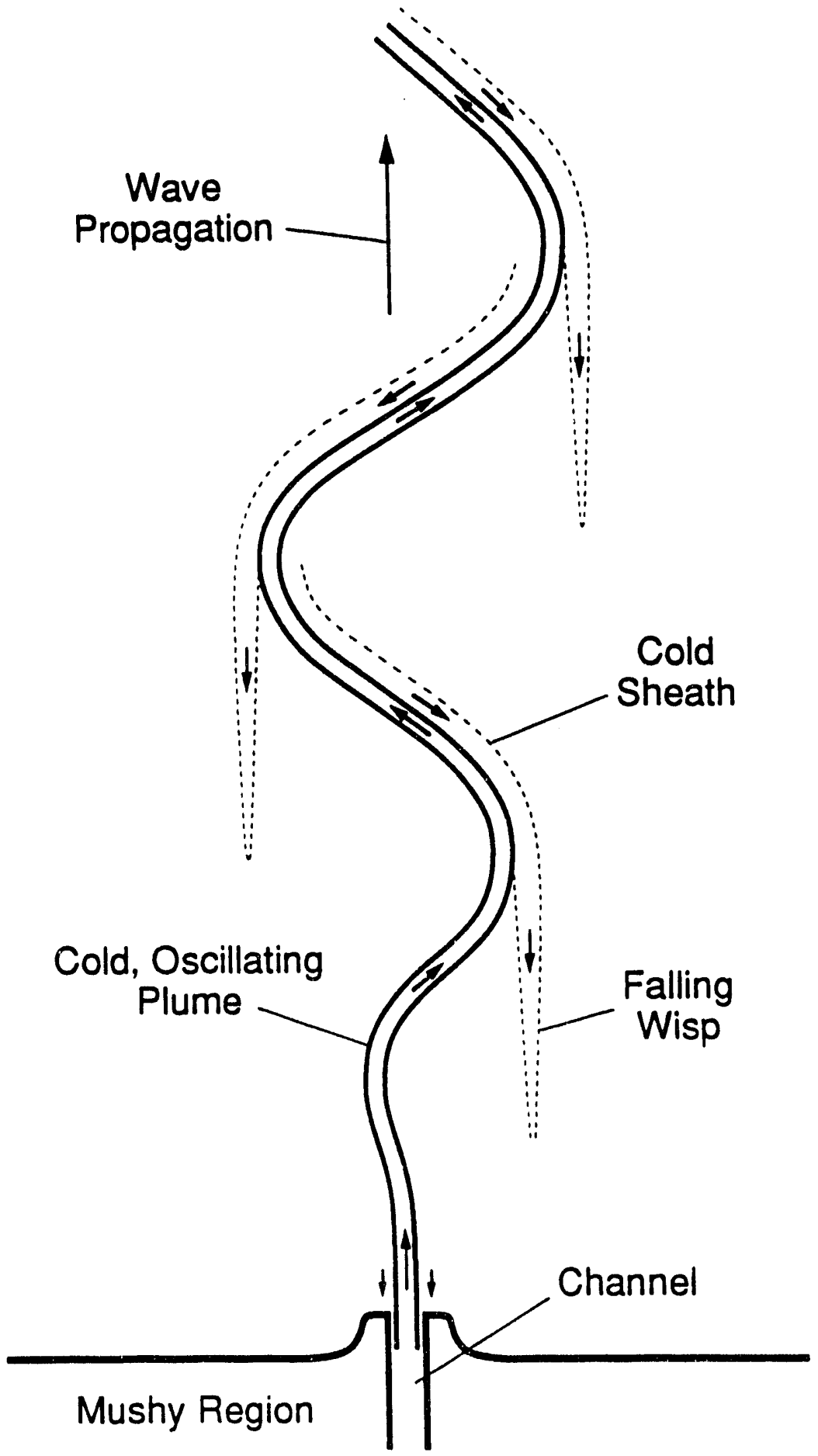

Figure 4.9 jchematic showing flow patterns adjacent to an oscillating plume. 


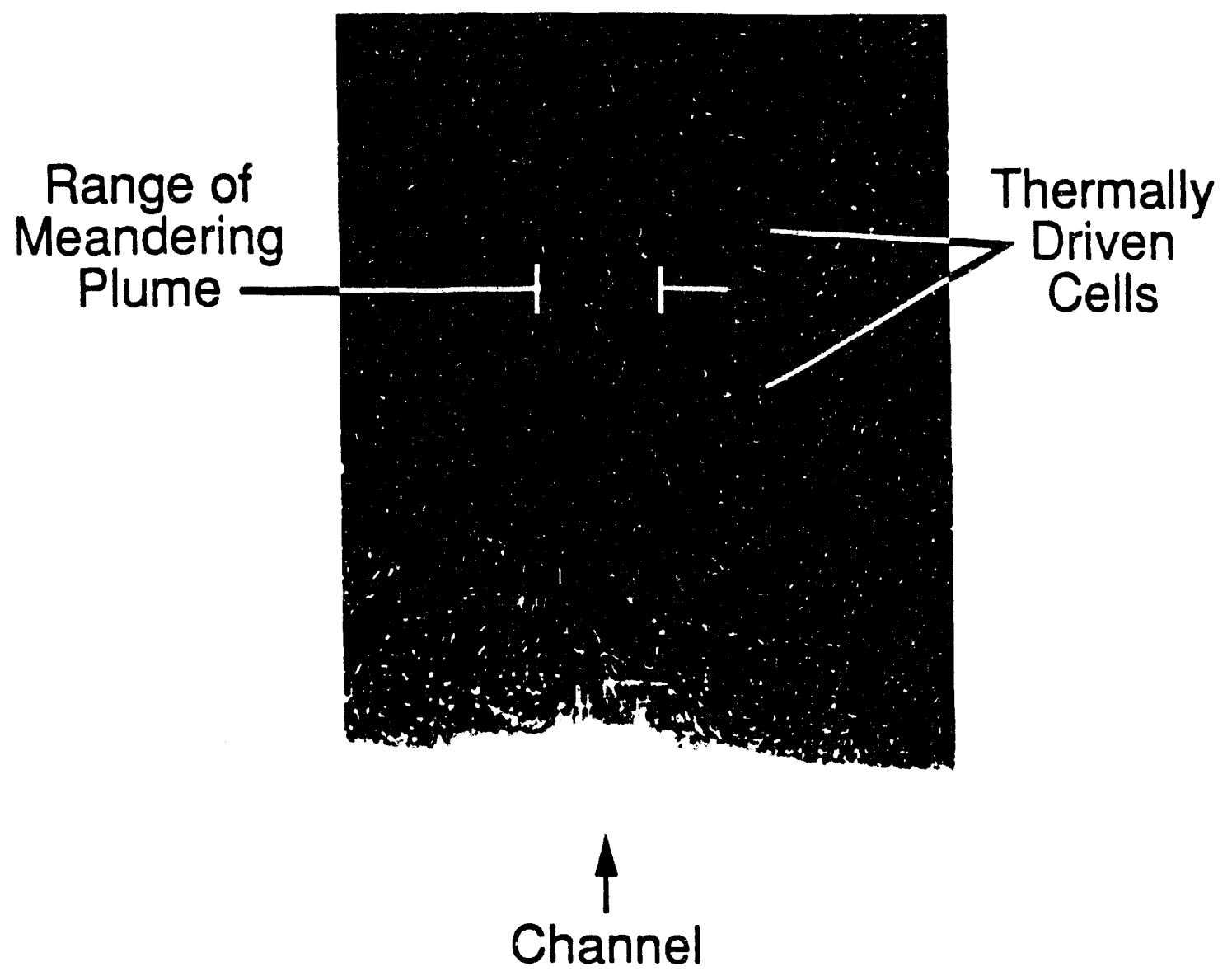

Figure 4.10 Particle tracking with time exposure of 3 seconds showing cellular flows around an ascending, meandering plume. 


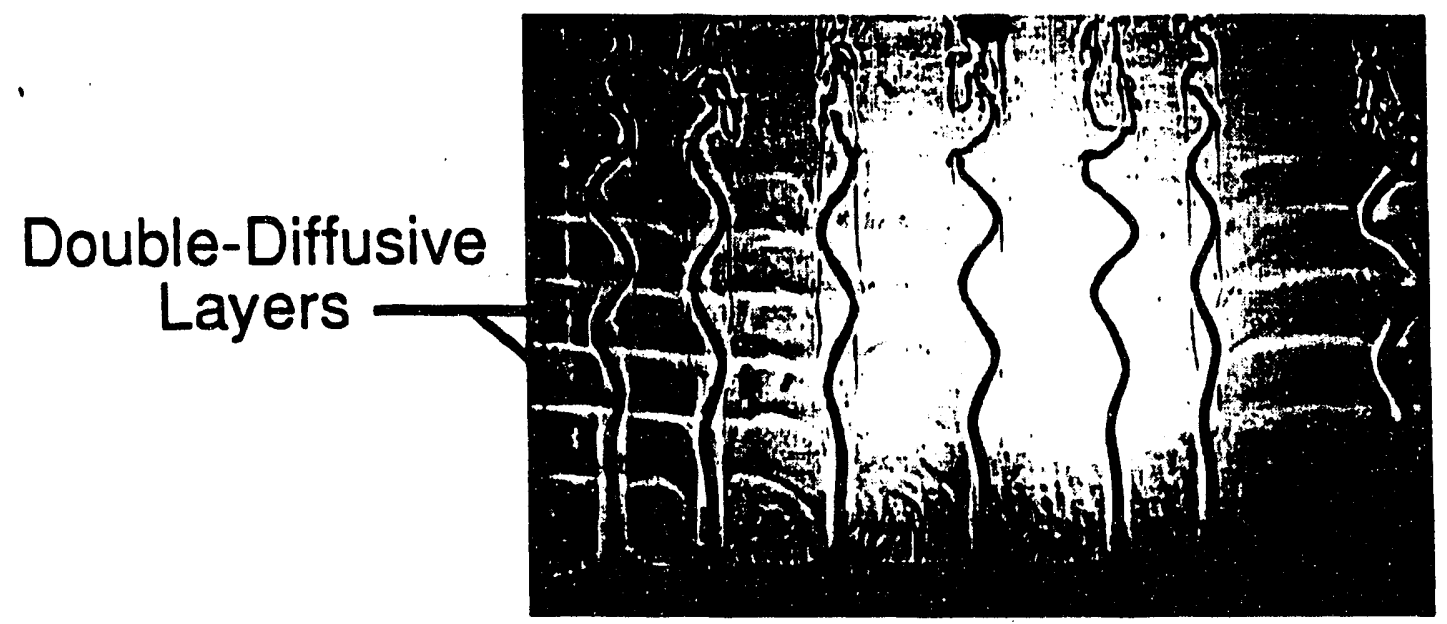

(a)

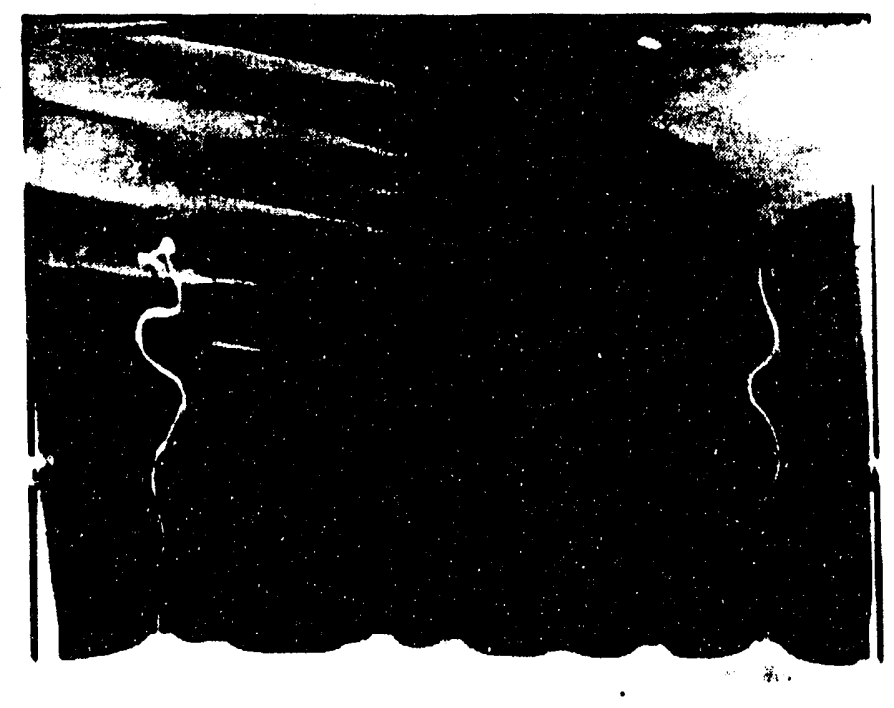

(b)

Figure 4.11 Shadowgraph of double-diffusive layers in the melt of a UDS system at approximately 2 hours. 


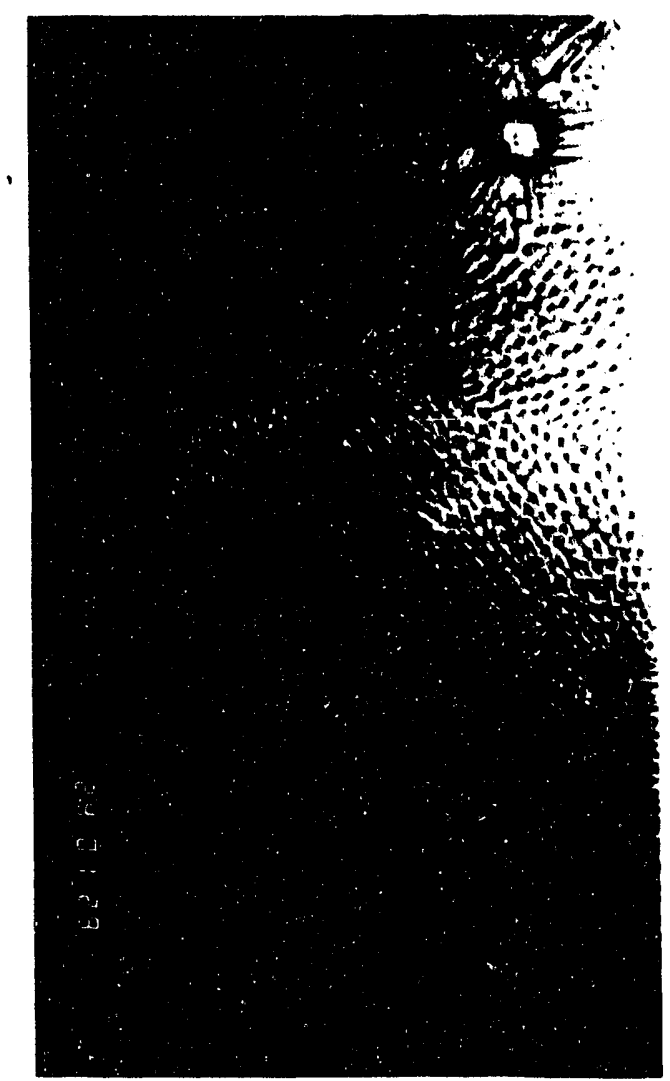

(a)

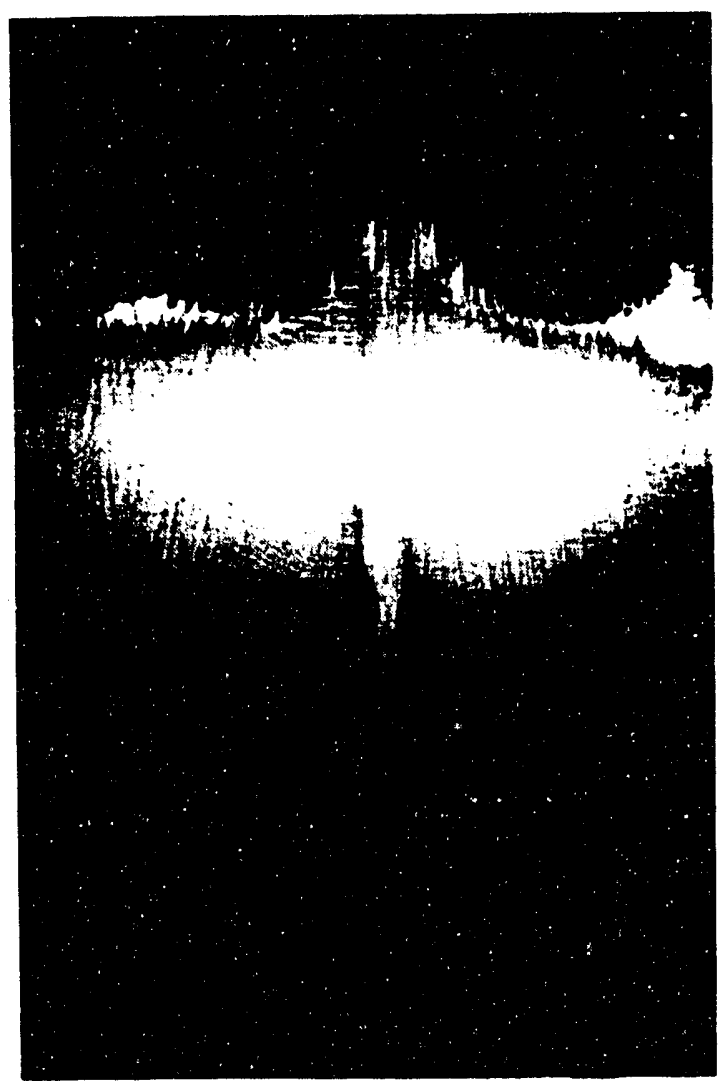

(b)

Figure 4.12 Crystalline grain structure of mushy region and channels from (a) top, and (b) side. 
dendrite is aligned in a particular orientation. Such a region is commonly referred to as a grain, and the dendrites that occupy a grain are themselves part of a single crystal. Channels that develop in the mushy region disrupt the grain structure, thereby adversely affecting the strength of the cast. Figure $4.12 \mathrm{~b}$ shows, in addition to the grains, the internal structure of a channel. The salient feature of the photograph is the overgrown and disorganized crystal growth immediately surrounding the channel. It is this noncolumnar crystal sheath and the eutectic material which eventually forms the freckle that is so deleterious to the strength of the final cast.

With increasing time, growth of the mushy zone reduced heat transfer to the chilled bottom, thereby reducing the solidification rate and the supply of water-rich interdendritic fluid to the channels. Hence, there was a progressive reduction in the number of plumes. When the supply of fluid flowing through a channel ceased, the channel quickly closed and became overgrown with crystals from the surrounding mush. At approximately two hours and thirty minutes into the experiment, only two weak plumes remained, and double-diffusive layers also became less distinct, with attendant weakening of the convection cells. This behavior confirms the dependence of doublediffusive layering and cellular flow on the existence of strong plume motion. Due to diminishing convection effects, all experiments were stopped shortly thereafter.

\subsubsection{Quantitative Measurements}

Using an automated data acquisition unit, temperatures were recorded at selected heights every 15 seconds during the course of an experiment. Related temperature histories are shown in Figure 4.13, and multiple experiments confirmed that the results were highly repeatable. Temperatures at all locations within the cast decreased in a relatively consistent manner, with the temperature nearest the heat exchanger dropping most quickly. As coolant chilled the heat exchanger, fluid just above the cold wall was 


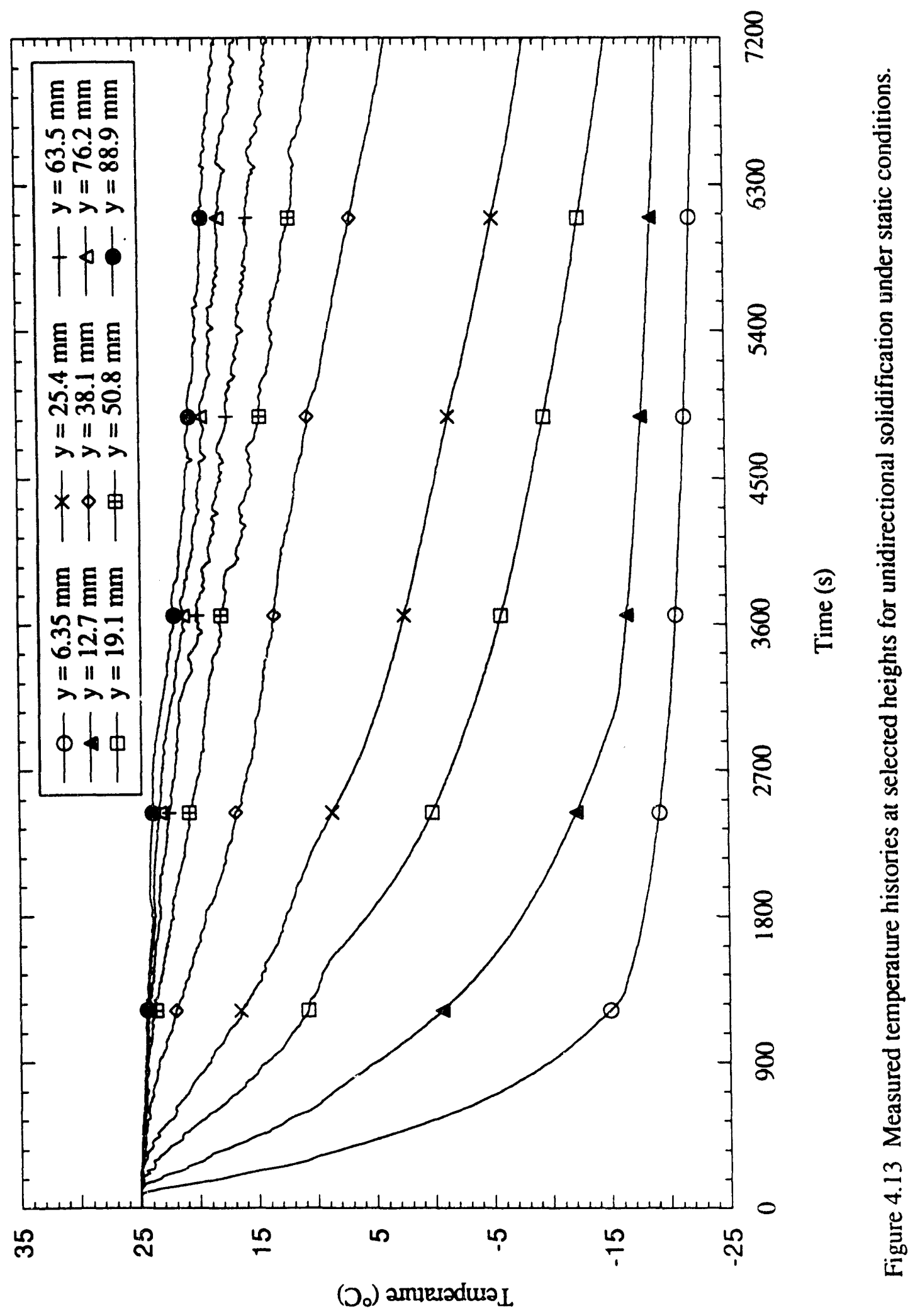


rapidly cooled through conduction dominated heat transfer, and at approximately 2 minutes, the temperature at $\mathrm{y}=6.35 \mathrm{~mm}$ had dropped to $18.6^{\circ} \mathrm{C}$, which corresponds to the liquidus temperature of aqueous $27 \% \mathrm{NH}_{4} \mathrm{Cl}$. Visual observations confirmed that, at $\mathrm{t}$ $=2 \mathrm{~min}$, the liquidus interface had, in fact, advanced to the lowest probe location. The temperature at $y=6.35 \mathrm{~mm}$ continued to decrease rapidly until the solidus front overgrew the lower probe at approximately 21 minutes. A discontinuity in the slope of the temperature history occurs when the solidus overgrows the probe. Salt fingering and plumes produced random temperature fluctuations in the melt during the later stages of solidification, which were no larger than $0.5^{\circ} \mathrm{C}$. However, as a probe was overgrown by the liquidus interface, the temperature fluctuations were effectively suppressed by the D'Arcy damping associated with the porous mushy region.

Figure 4.14 shows melt species concentration measurements for static unidirectional solidification. Although the overall trends were repeatable, the actual concentration measurements varied from experiment to experiment. Ascending plumes carried water-rich fluid to the top of the test section, creating solutal gradients and stratifying the melt. This stratification is manifested in Figure 4.14, as concentrations at y $=76.2 \mathrm{~mm}$ were consistently more water-rich than those at $\mathrm{y}=50.8 \mathrm{~mm}$. The concentration decreased with time at both locations, and the melt attains a value just below $24 \%$ wt. $\mathrm{NH}_{4} \mathrm{Cl}$ at the conclusion of the experiment.

\subsection{Side and Bottom Wall Chilling}

\subsubsection{Qualitative Results}

Almost immediately after applying coolant to the heat exchangers of test section II, thin boundary layers of cold fluid descended along the two chilled side walls, inducing two thermal convection cells within the test section. As in the bottom cooling case (UDS), crystals began to form within 15 secunds. However, the majority of the first 


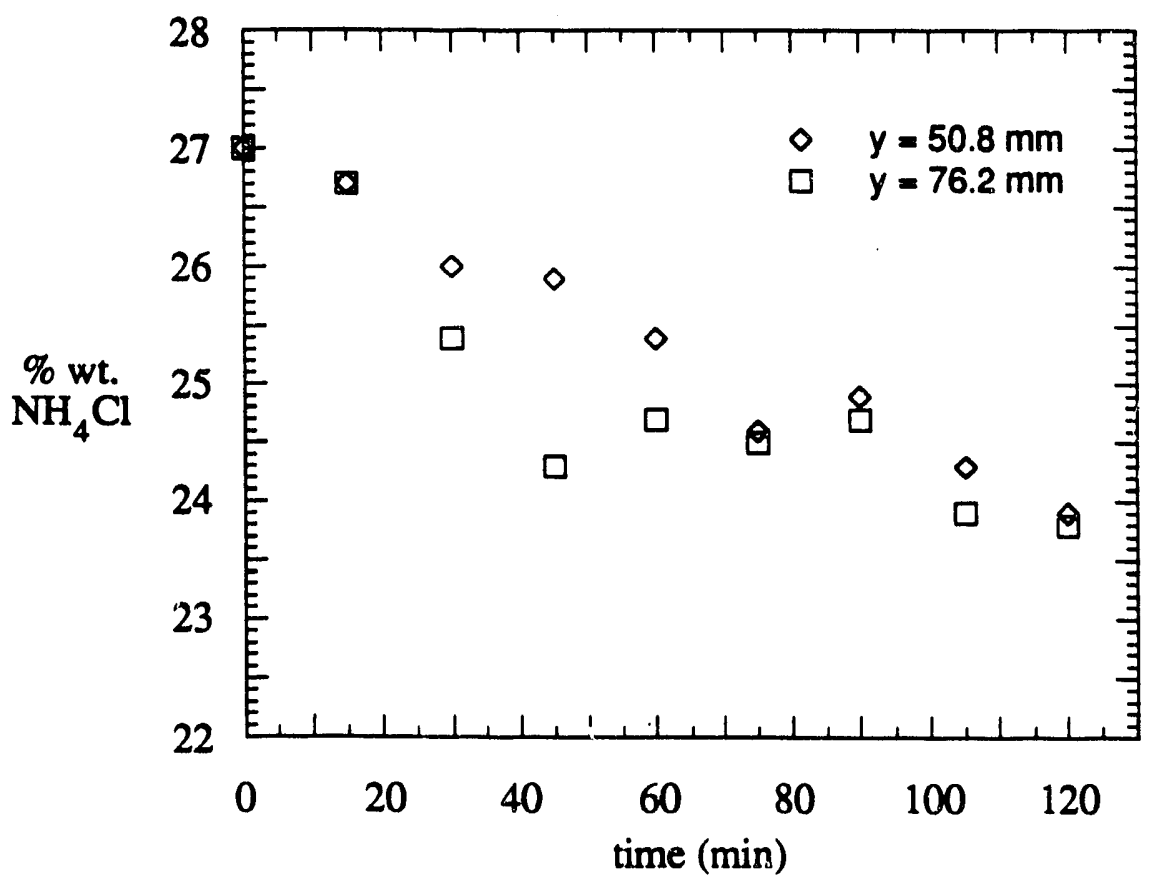

Figure 4.14 Liquid composition of ammonium chloride during unidirectional solidification under static conditions. 
crystals forming on the side walls detached from the walls and descended to the cavity floor, collecting in two equiaxed mounds (Fig. 4.15b). Along the bottom wall, crystal growth was equally vigorous, and salt finger formation was again very pronounced. The equiaxed mounds provided preferred sites for crystal growth along the liquidus front associated with solidification from the bottom cold plate. These elevated sites grew rapidly and rejected large amounts of water-rich fluid, which, in turn, formed vigorous plumes early in the process.

Subsequently, a substantial mushy region established itself on all walls, and related solutal buoyancy effects became apparent about two minutes into the experiment. Water-rich fluid ejected from the side wall mushy region became entrained in the falling thermal boundary layer adjacent to the liquidus interface. However, eventually, the water-rich fluid would emerge from the descending boundary layer and enter the bulk liquid, where it would begin to ascend in a plume-like manner. Figure $4.15 \mathrm{~b}$ shows the fishhook-like structure associated with this behavior. In addition to rejecting fluid at the liquidus interface, the sidewall mushy region delivered interdendritic fluid to the top of the test cell.

At about six minutes into the experiment, two pockets of near eutectic fluid began to form at the upper corners of the melt (Fig. 4.15d). As these two fluid pockets grew, they eventually joined at the top center of the test section, forming the first of several double-diffusive convection cells. Moreover, as this top cell developed, the adjacent mushy regions remelted due to a reduction in the liquidus temperature associated with increased concentration of eutectic fluid in the convection cell.

After ten minutes, a small collection of crystals began to coalesce in the upper corners of the melt, just below the newly formed convection cell (Fig. 4.16b). This slurry region consisted of a large number of tiny dendrites, which are believed to have been fractured from the crystal structure by the increasingly robust solutal upflow within the 


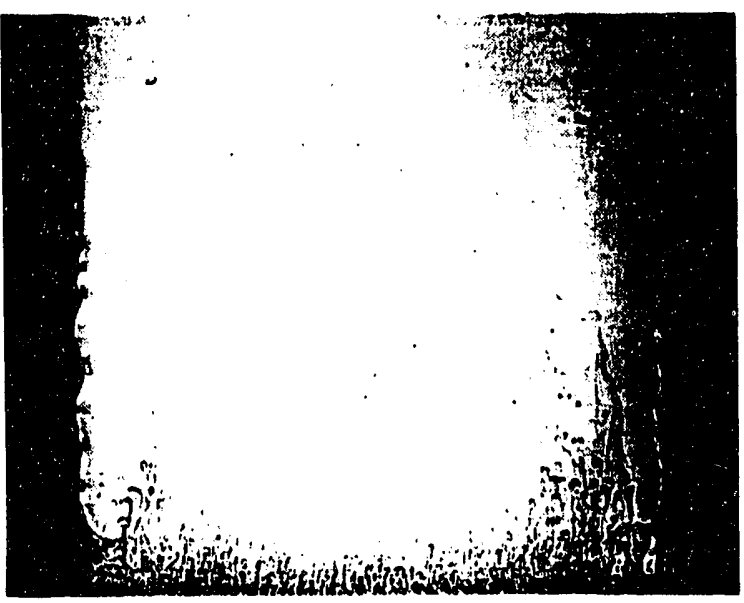

(a)

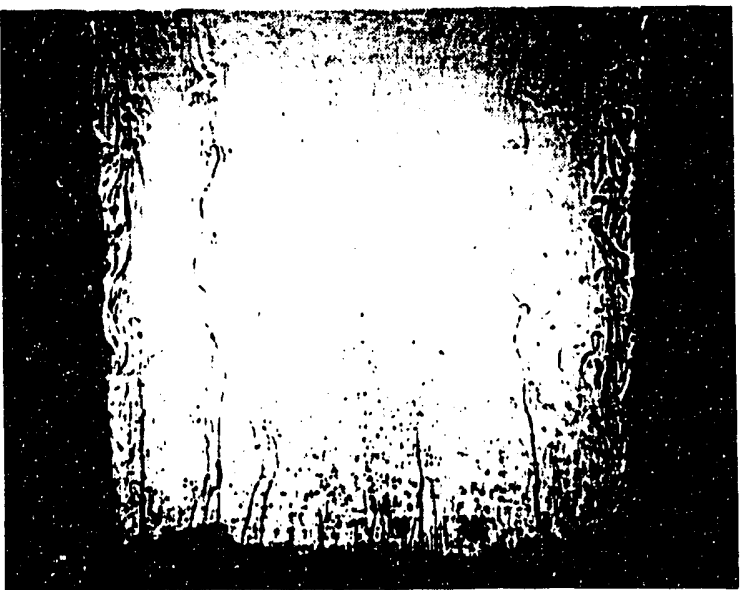

(c)

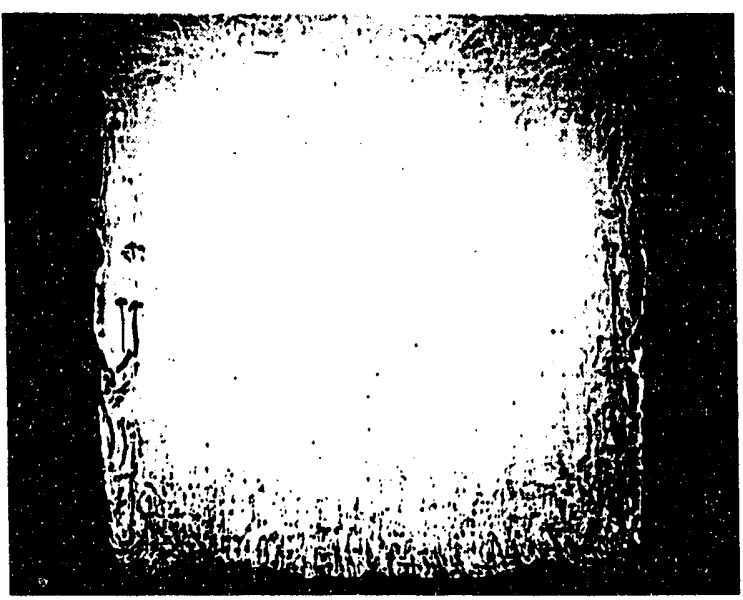

(b)

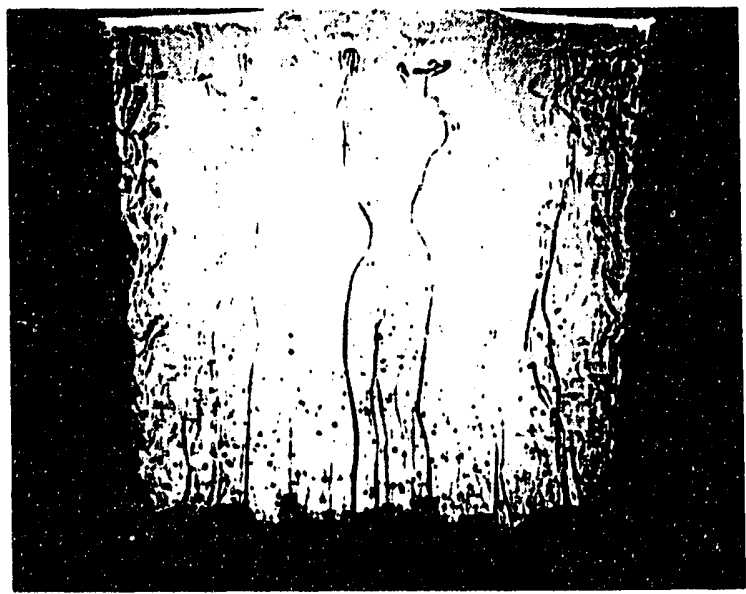

(d)

Figure 4.15 Shadowgraph of early solidification for concurrent side and bottom wall chilling: (a) 1 , (b) 2.5 , (c) 5 , and (d) $7.5 \mathrm{~min}$. 


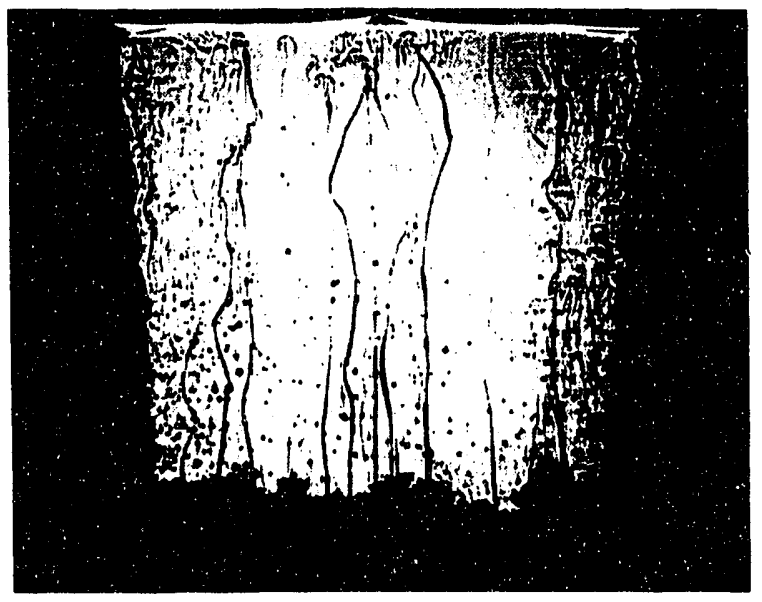

(a)

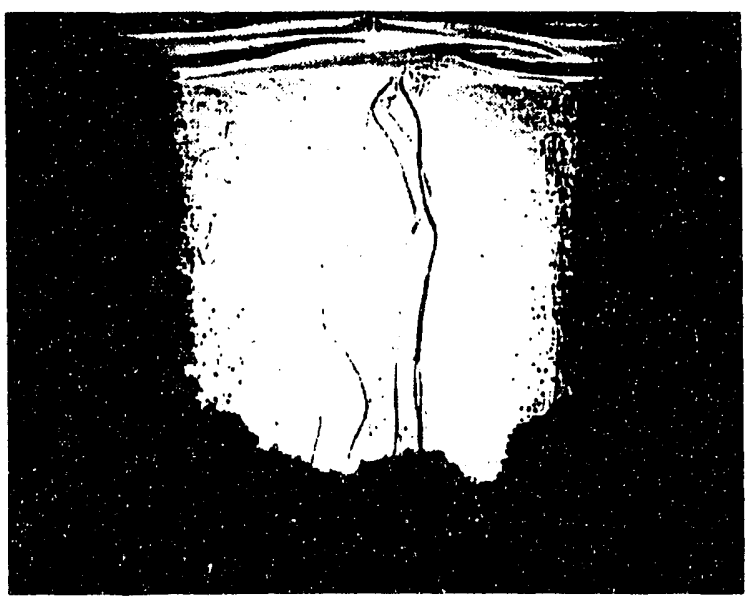

(c)

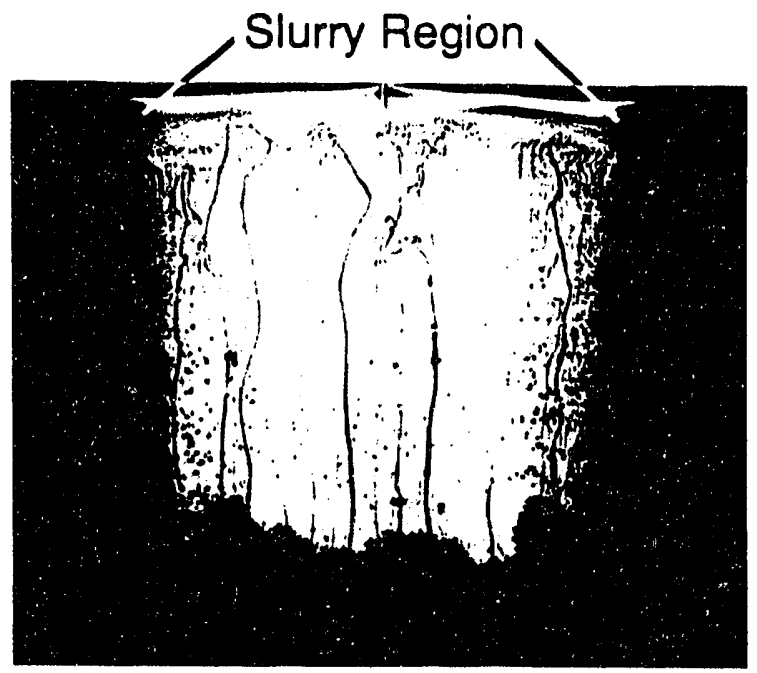

(b)

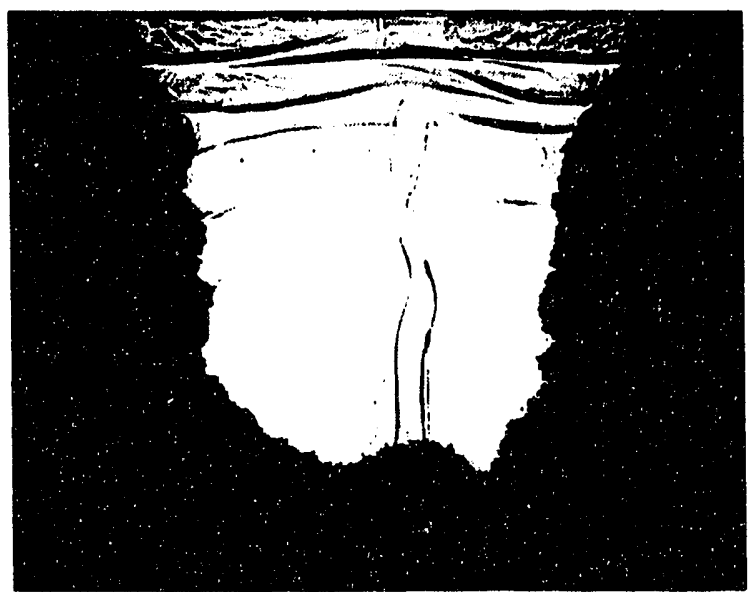

(d)

Figure 4.16 Shadowgraph showing double-diffusive cell development, rapid growth of the liquidus front, and crystal ejection in the form of slurries: (a) 10 , (b) 12.5 , (c) 15 , and (d) $20 \mathrm{~min}$. 
mush. Due to shear forces generated by the upper convection cell, the dendrites were dragged a short distance into the bulk liquid, while slowly falling, and were eventually entrained by the ever present downflow of fluid adjacent to the liquidus front. Juxtaposing a shadowgraph image and a particle tracking photograph, Figure 4.17 reveals slurry behavior, as well as the A-segregate type channels from which the slurry was ejected. As they fell, the crystals grew and subsequently became lodged in the mushy region along the side wall or fell to the cavity floor, thereby enhancing crystal growth along both the bottom and sides. Along the cavity floor, the equiaxed mounds continued to grow, providing robust plumes that vigorously mixed the melt. The plumes also advected large numbers of free floating dendrite crystals high into the melt, and crystals that descended back to the mush sustained equiaxed growth conditions in the lower regions. Owing to the large number of crystals ejected into the melt, nucleation also occurred on the Lexan cavity wall, immediately above the liquidus front growing from the bottom (Fig. 4.15c). Shortly thereafter, these nucleation sites were overgrown by the mushy zone.

Previously, it was indicated that crystals in the slurry originated either from broken dendrite tips or from crystals that nucleated within the interstices of dendrites. A comparison of results from the UDS and side-cooled conditions suggests that the slurry zone consists primarily of broken dendrite tips. Under UDS conditions, salt fingers initially move in the direction of columnar dendrite growth. Therefore, very few dendrite tips are broken. Conversely, for side-wall cooling, interdendritic fluid flows across the dendrite structure, enhancing prospects for tip fracture and ejection at the top of the test section to form the slurry. This slurry also represents the early stages of the side wall Asegregate type channels.

After the first convection cell developed at the top of the test section, another cell formed just below it (Fig. 4.16c, d). Such cells continued to form, until the melt 


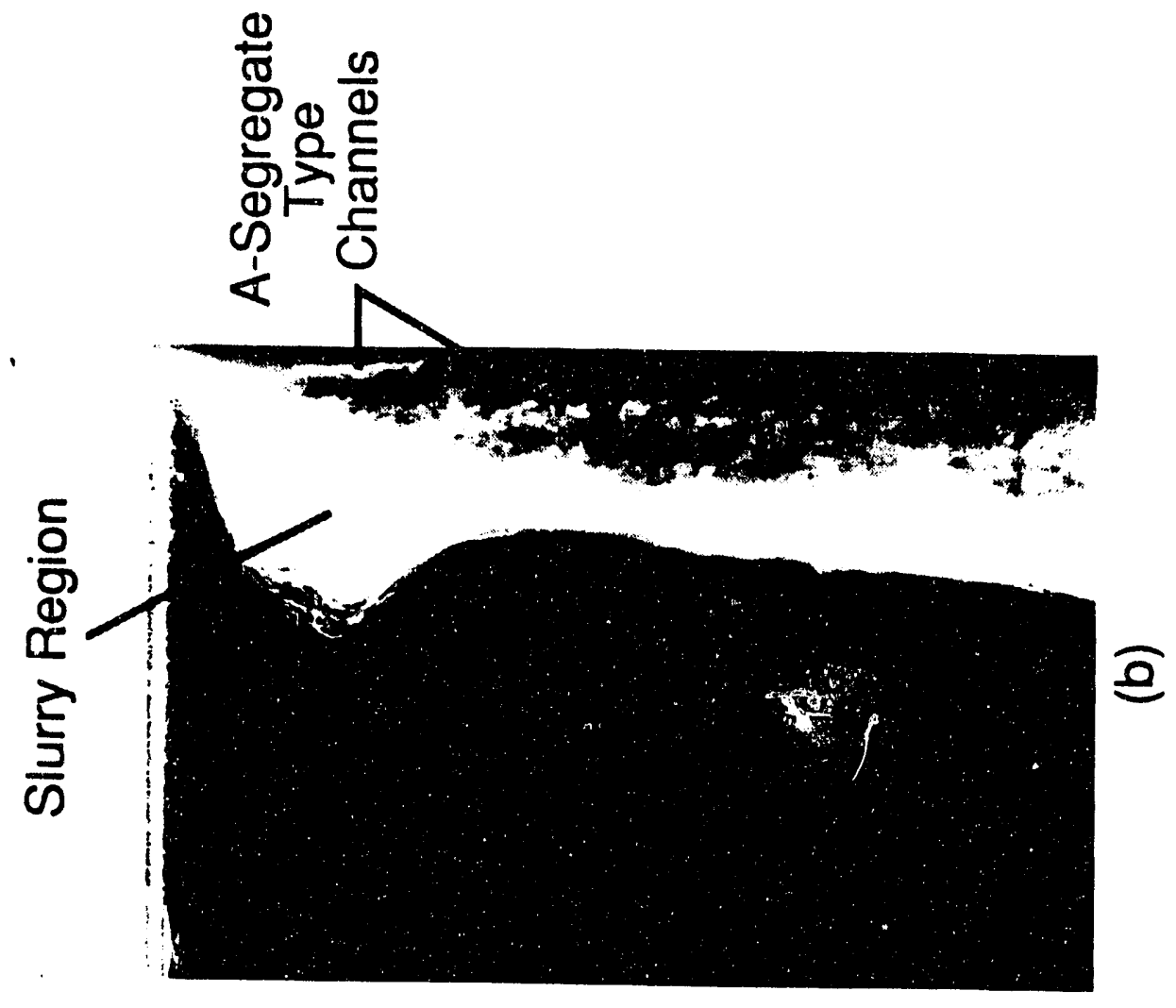

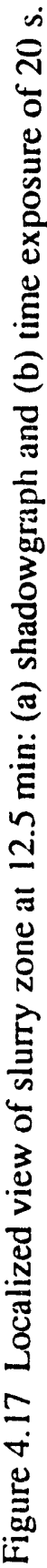

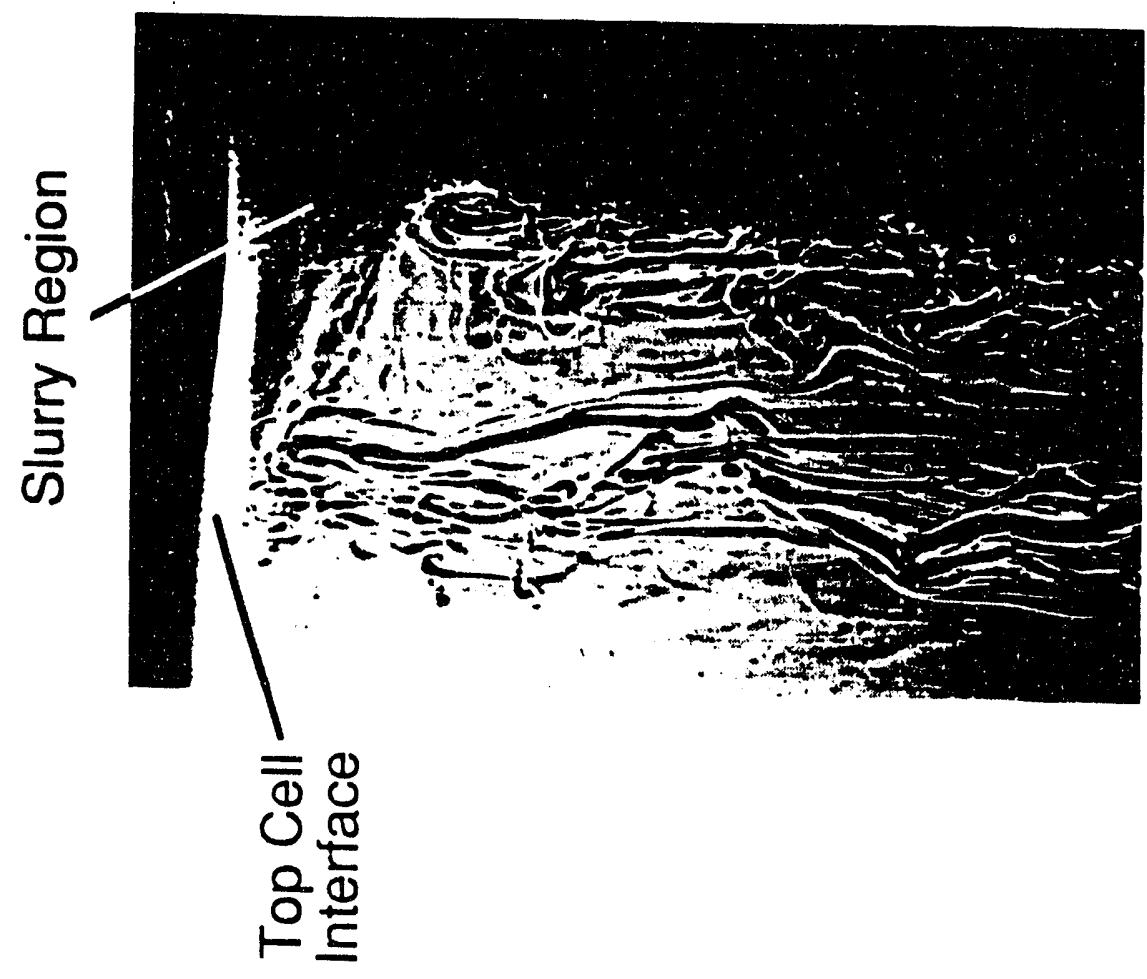

(્) 
consisted of multiple double-diffusive convection cells, as shown in Figure 4.18. Each cell grew with time and had a distinct fluid composition. Owing to the absence of a mushy region, the top cell was observed to be of nearly eutectic composition and each of the underlying cells consisted of increasingly higher $\mathrm{NH}_{4} \mathrm{Cl}$ concentrations, such that fluid in the bottom of the melt was closest to the original $27 \% \mathrm{NH}_{4} \mathrm{Cl}$ mixture. As evidenced by the tendency toward condensation on the outer surface of the test chamber, the top cell also was the coldest of the double-diffusive cells. Because it was fed by nearly eutectic fluid rejected at the interface of the solid and mushy regions, where, according to the phase diagram (Fig. 2.4), the temperature is $-15.4^{\circ} \mathrm{C}$, this observation is not surprising. Fluid motion within each cell was driven by thermal buoyancy, with downflow occurring along the vertical crystalline interfaces. The cellular flow pattern is shown in greater detail in Figure 4.19, and the largest velocities within the cells were on the order of $1 \mathrm{~mm} / \mathrm{s}$. In their development stages, convection cells would not flow horizontally along the bottom of the layer to the center of the test section but, due to heating by the bulk fluid, would tilt upwards toward the top of the layer. Shear forces induced by the momentum associated with this upflow created a weaker, counter-rotating companion vortex. However, the companion cell was short lived, as the major cell eventually grew to occupy the entire horizontal layer.

The initial formation of double-diffusive convection cells was primarily associated with the ejection of eutectic and nearly eutectic fluid from A-segregate type channels in the side wall mush (Fig. 4.20). Due to the large horizontal temperature gradient, the cells were characterized by strong interfaces and robust convection. Moreover, since they initially formed from an upwelling of interdendritic fluid within the side wall mush, the cells developed in concert with a dense slurry zone.

Fluid motion within a cell was augmented by fluid rejection from A-segregate channels that developed in the side wall mushy region. These channels acted as paths of 


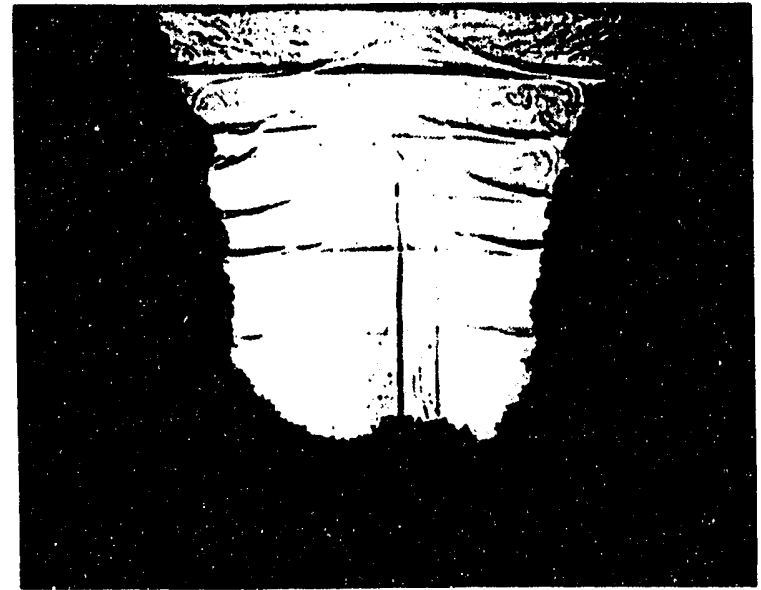

(a)

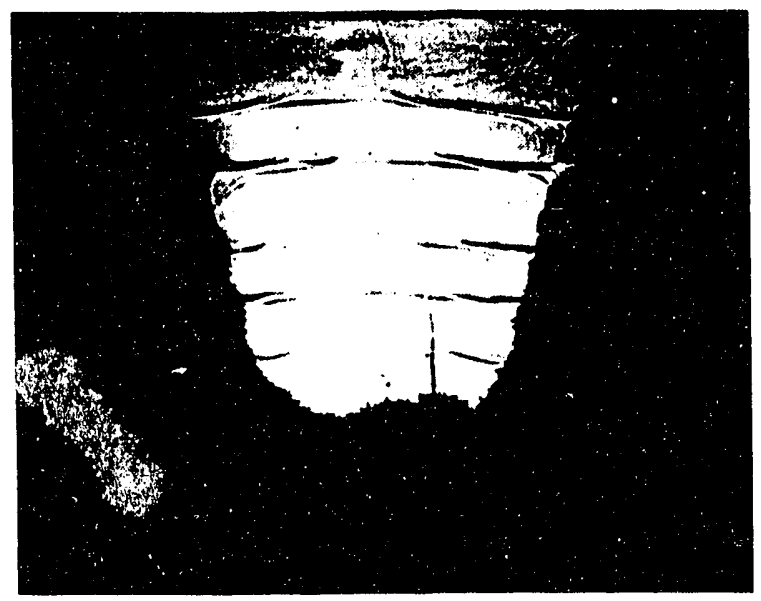

(c)

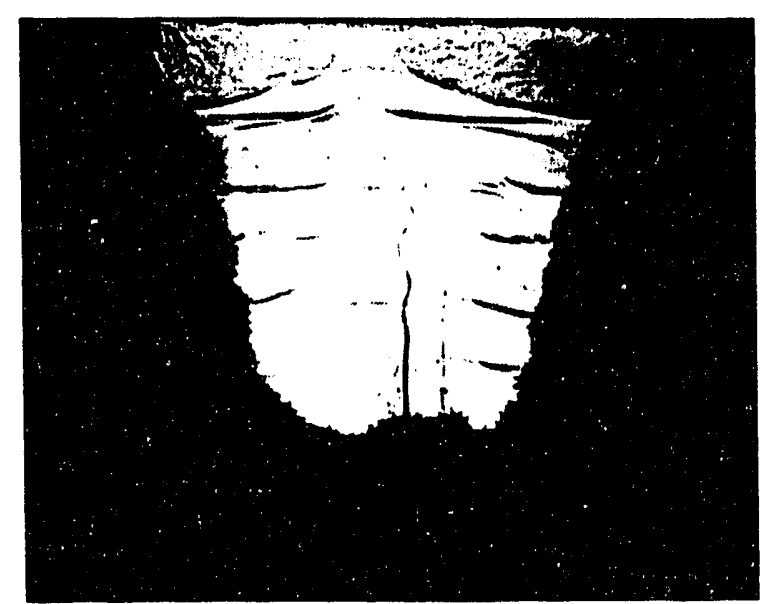

(b)

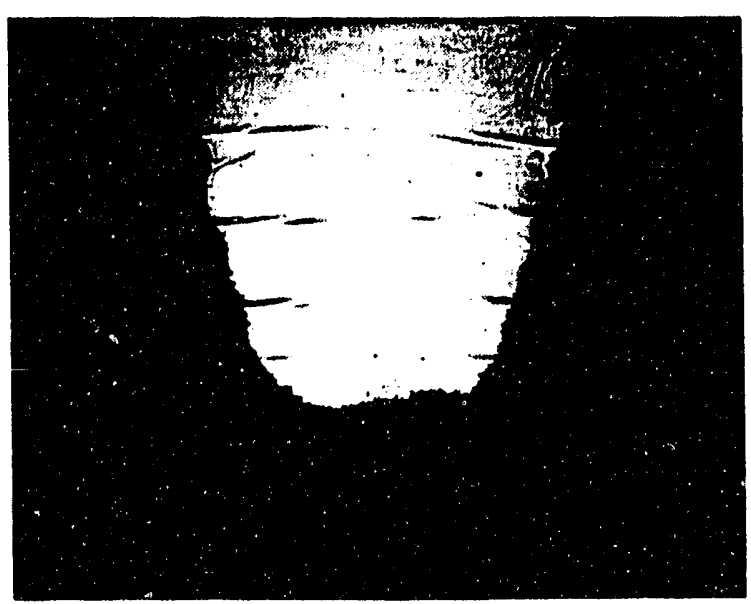

(d)

Figure 4.18 Shadowgraph of late development showing advancing liquidus front, maturing convection cells, and impedance of ascending plumes by the cells: (a) 30, (b) 40, (c) 50, and (d) $60 \mathrm{~min}$. Note: upper convection cell is free of a mushy region. 

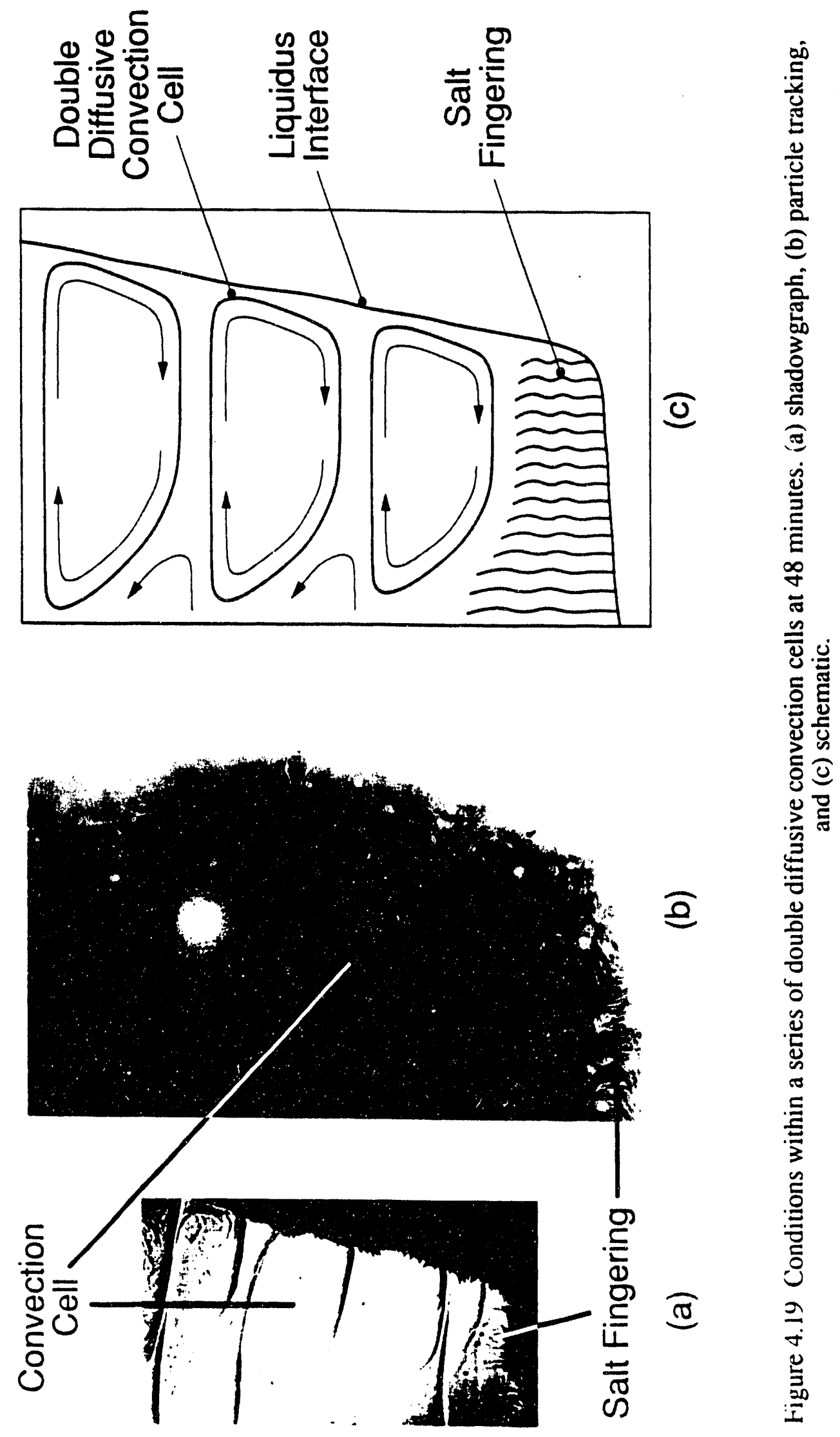


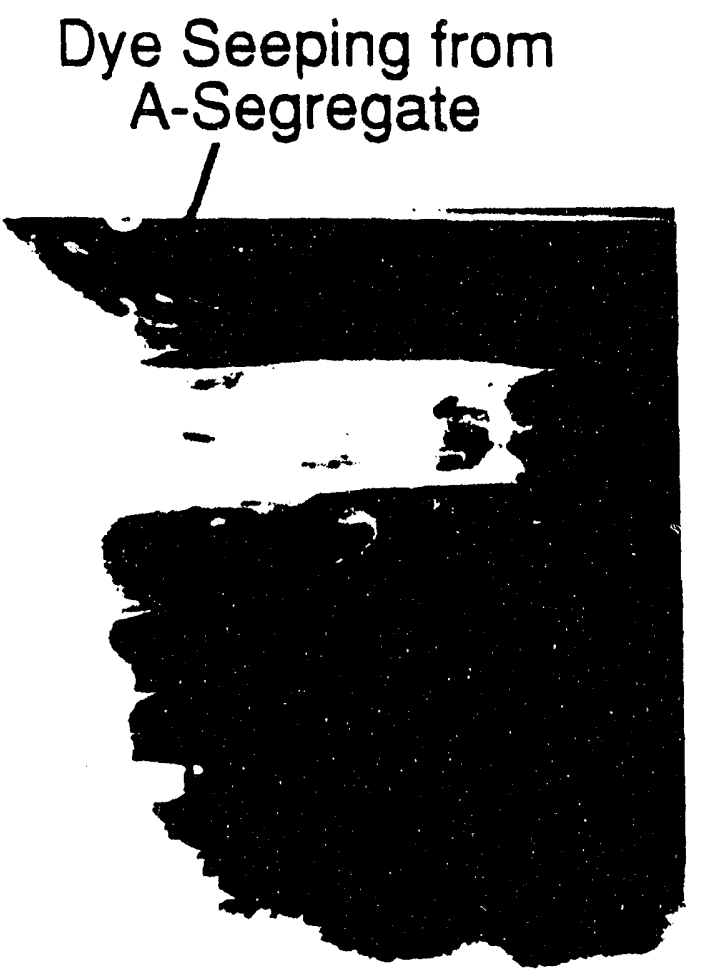

Figure 4.20 Fluorescein dye injection into side wall mushy region at 45 minutes showing double-diffusive cells in the melt as well as a plume emanating from an Asegregate type channel. 
least resistance for interdendritic fluid ascending from the mushy region and tended to eject fluid at the bottom of established convection cells, thereby enhancing cell motion. Although A-segregate channels form as individual holes or pencil-like voids in the mush, several of the channels tended to form close to each other across the thickness of the test cell. With such an aggregation of channels in the mush, nearly two-dimensional fluid motion was established within the melt. However, plumes rising from volcanos along the bottom remained uniquely three dimensional.

Unlike the UDS case, plumes that originated from the bottom of the mold in this arrangement were impeded in their upward movement. The plumes rose only as far as their solutal buoyancy permitted. As seen in Figure 4.18, plumes would rise until they achieved equilibrium with a double-diffusive convection cell of comparable density. As illustrated in Figure 4.16 by the plume on the far right, some plumes that initially formed on the bottom, relatively close to the side walls, were enveloped by the advancing side wall liquidus front. However, rather than terminating the plume, the side wall liquidus front actually grew around the plume, essentially transforming it into an A-segregate channel for the side wall mushy region.

Beyond one hour, the solidification rate dropped significantly and the doublediffusive convection cells continued to grow downwards. At two hours, two weak layers remained, each accounting for about one-half of the melt. The upper layer consisted of eutectic fluid while the bottom layer was nearly eutectic, as suggested by the existence of a relatively small adjoining mushy zone. All experiments were stopped at two hours.

\subsubsection{Quantitative Measurements}

Observations made during flow visualization for test section II suggested some general trends pertaining to temperature and concentration fields. For example, the presence of stable, double-diffusive layers and differences in mushy region thicknesses 
indicated a solutal stratification, with water-rich fluid existing near the top of the melt and salt-rich fluid near the bottom. Similarly, condensation on the outside viewing surface of test section II revealed a temperature inversion in the melt, which developed over time. Quantitative studies were therefore undertaken to characterize temperature and liquid concentration associated with side and bottom cooling.

Temperature measurements were taken every 15 seconds at selected locations within test section II (Fig. 2.5). Thermocouple locations were chosen to study temperature characteristics along one vertical array and two horizontal arrays. Figure 4.21 shows the cooling curves along the vertical thermocouple array placed $12.7 \mathrm{~mm}$ off the centerline of the test section. Unlike cooling curves presented for UDS, the first three minutes of side and bottom cooling solidification were characterized by a thermal plateau from which temperatures successively decreased as time progressed. As an experiment was started, thermal buoyancy produced thin boundary layers of cold, falling fluid at the vertical walls, which eventually collected at the bottom of the cavity. Continuity dictates that fluid at the original melt temperature must slowly ascend. As time progressed, a pool of cold fluid collected at the bottom the cavity, and the pool successively influenced the thermocouples above it, until, at approximately two minutes, the upper-most probe temperature began to drop from the plateau.

The temperature inversion suspected during flow visualization experiments is verified by the cooling curves. In fact, the cooling curves clearly show that, toward the end of the experiment, the warmest location in the melt was in the lower, center section of the cavity, just below the liquidus interface (Fig. 4.21). Over the course of an experiment, light, albeit cold, fluid was continually being rejected to the top of the test cavity from the side wall mushy region. Warm, salt-rich fluid was therefore forced downward to the lowest regions of the melt, creating the thermal inversion. 


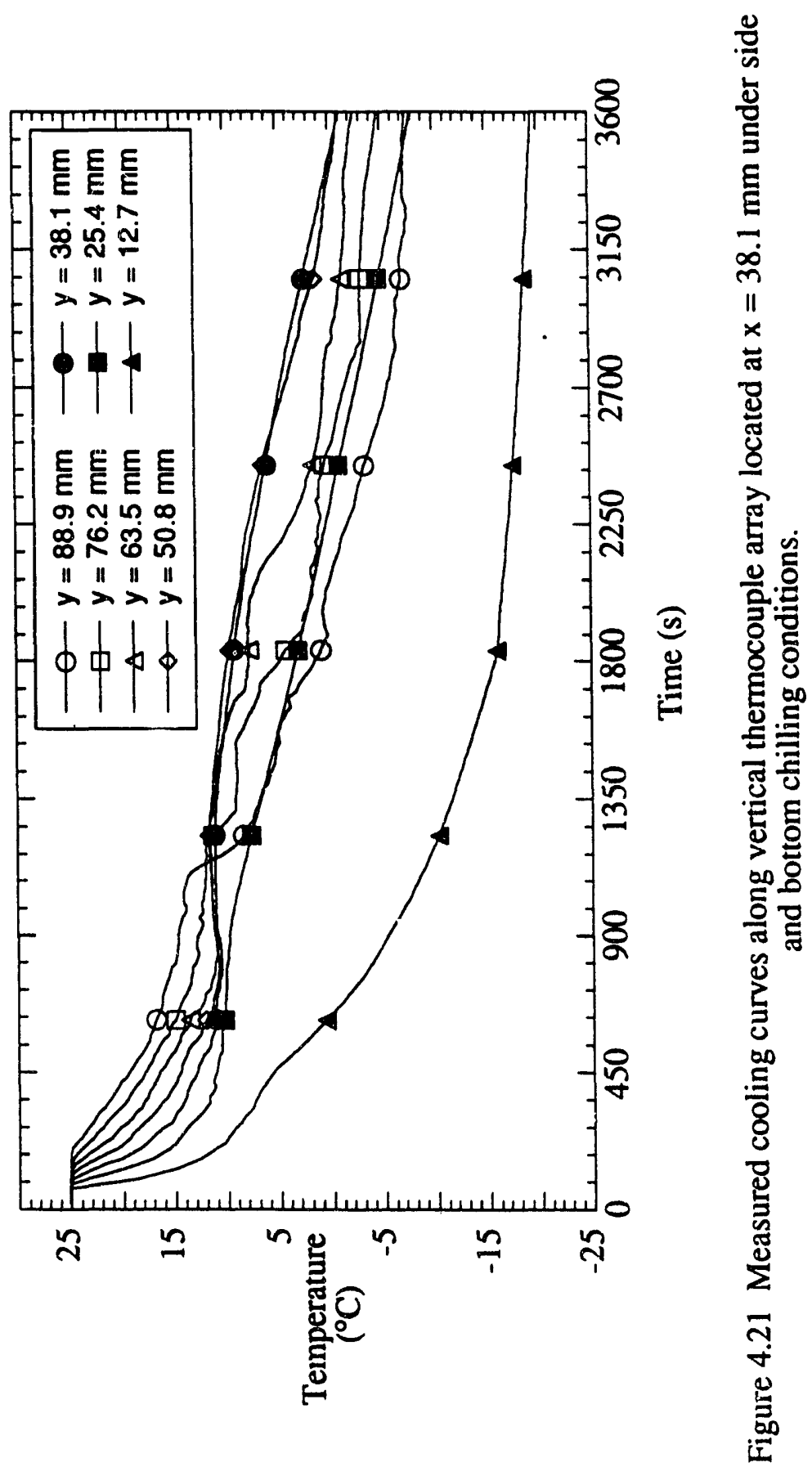


Double-diffusive interfaces (Fig 4.18) separated cells of thermally and solutally disparate fluid, and the downward propagation of double-diffusive cells is manifested in the cooling curves by rapid drops in temperature (Fig. 4.21). Between the passages of interfaces, temperature profiles within the cells were relatively flat indicating that each cell was generally well mixed and isothermal. The downward propagation of the convection cells produced a step-like pattern in the cooling curves; however, as the cells weakened in the later stages of an experiment, the stepping pattern became smoother. The stepped vertical temperature profile therefore depended on cell strength.

Figure 4.22 shows temperature histories obtained by the two horizontal arrays. Both arrays indicated nearly isothermal conditions, until approximately ten minutes, at which time, temperatures at the inner locations no longer decayed at a rapid rate and remained approximately constant for nearly seven minutes. In fact, the temperatures of the lower array (Fig. 4.22b) actually rose slightly, as warm fluid was pushed downward by developing double-diffusive cells. At approximately 20 minutes, the highest doublediffusive interface crossed the upper array, generating a temperature drop. Within a particular double-diffusive cell, thermal convection carries chilled fluid away from the mushy region at the bottom of the cell and impinges the mushy region with warm fluid at the top of the cell. The measurements at $y=76.2 \mathrm{~mm}$ and $\mathrm{x}=25.4 \mathrm{~mm}$ confirm this phenomenon. Five millimeters from the liquidus front, this particular thermocouple was strongly influenced by thermally driven flow in a cell. As a double-diffusive interface passed the probe at $30 \mathrm{~min}$, the probe entered the bottom of the upper double-diffusive cell where fluid chilled by the liquidus interface flowed inward and across the probe. As the cell progressed downward, the probe experienced an impingement of warm fluid in the upper section of the cell, with a rise in temperature followed immediately by a rapid temperature drop associated with the passing of yet another interface. In addition to the strong vertical thermal gradients present across the double-diffusive interfaces, there 


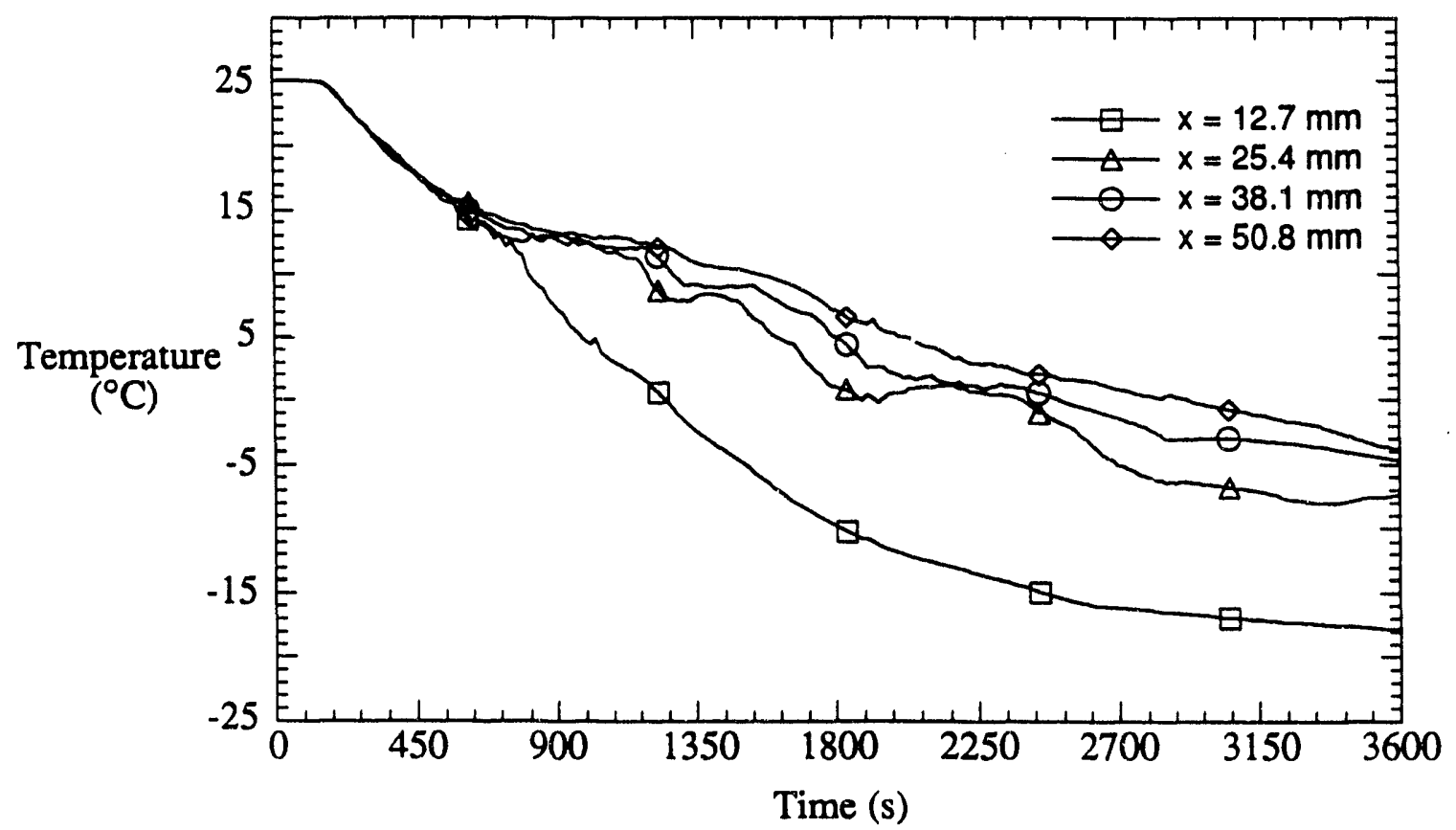

(a) Upper Array

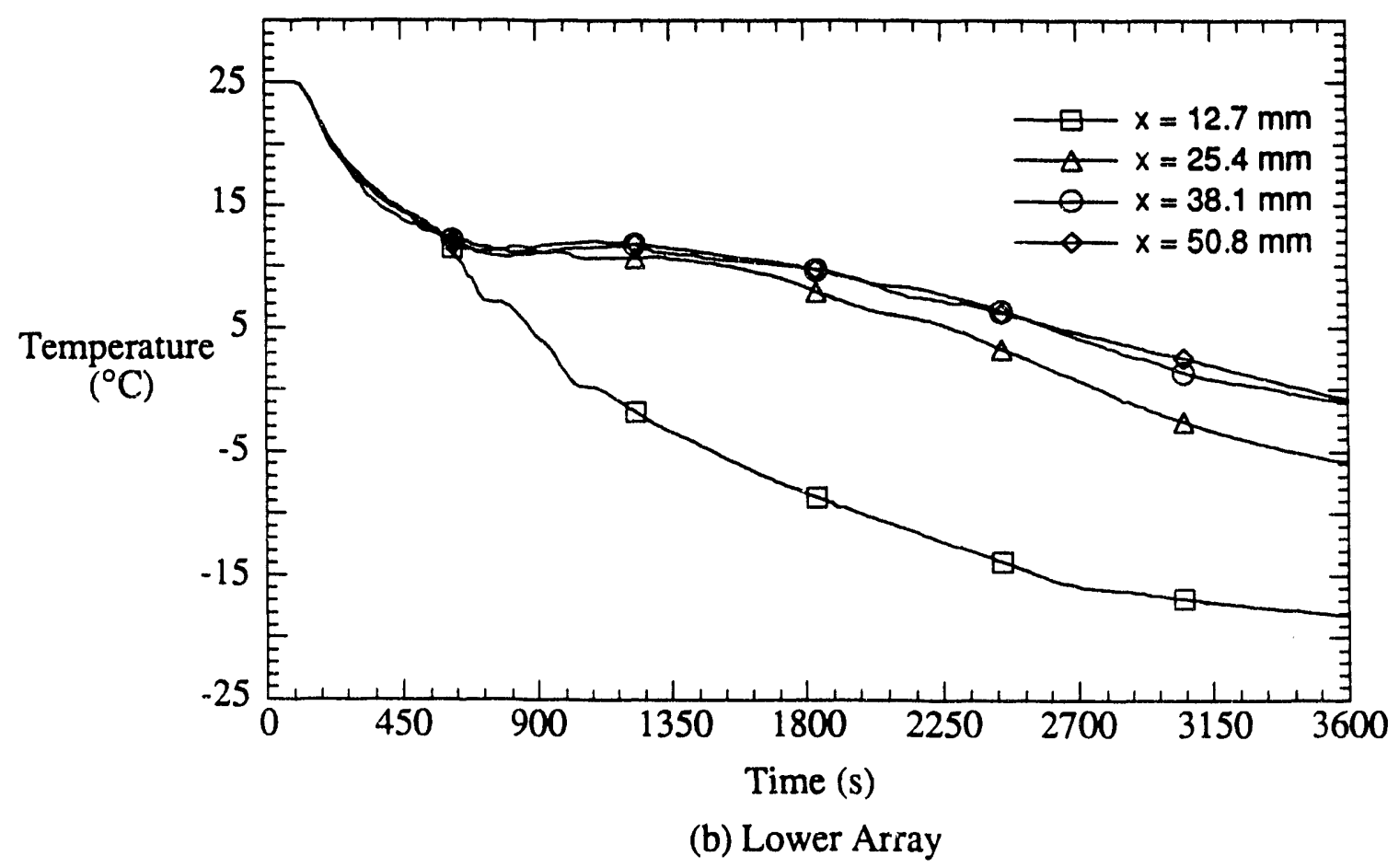

Figure 4.22 Measured cooling curves under side and bottom chilling conditions at (a) $y=76.2 \mathrm{~mm}$ and (b) $y=50.8 \mathrm{~mm}$. 
existed a slight horizontal thermal gradient across each cell. Owing to weakening doublediffusive cells, temperatures decayed a more smoothly toward the end of an experiment.

The measurements of mass fraction ammonium chloride support the foregoing premises. Five sets of concentration measurements from the prescribed locations (Fig. 2.5) were taken at $0,15,30,45$, and 60 minutes, and the resulting values can be seen in Figure 4.23. Owing to relatively efficient mixing by thermal convection, concentrations in the melt dropped consistently through the first 15 minutes, as the growing mushy region froze out $\mathrm{NH}_{4} \mathrm{Cl}$ and rejected water-rich solution. However, as double-diffusive convection began to dominate flow from 15 to 30 minutes (Figs. 4.16 and 4.17), rapid solutal stratification led to large, stable, vertical concentration gradients in the melt. The concentration difference between the lower center and the upper corner locations was on the order of $2.5 \%$ wt. $\mathrm{NH}_{4} \mathrm{Cl}$. In general, centerline concentrations were higher than values off-center, and upper concentrations were higher than lower values. Past 30 minutes, stratification continued as convection patterns died out and the melt stabilized. The final concentration in the lower corner was not determined because it had been overgrown by the mushy region, thereby producing erroneous results using the current method of solution extraction. 


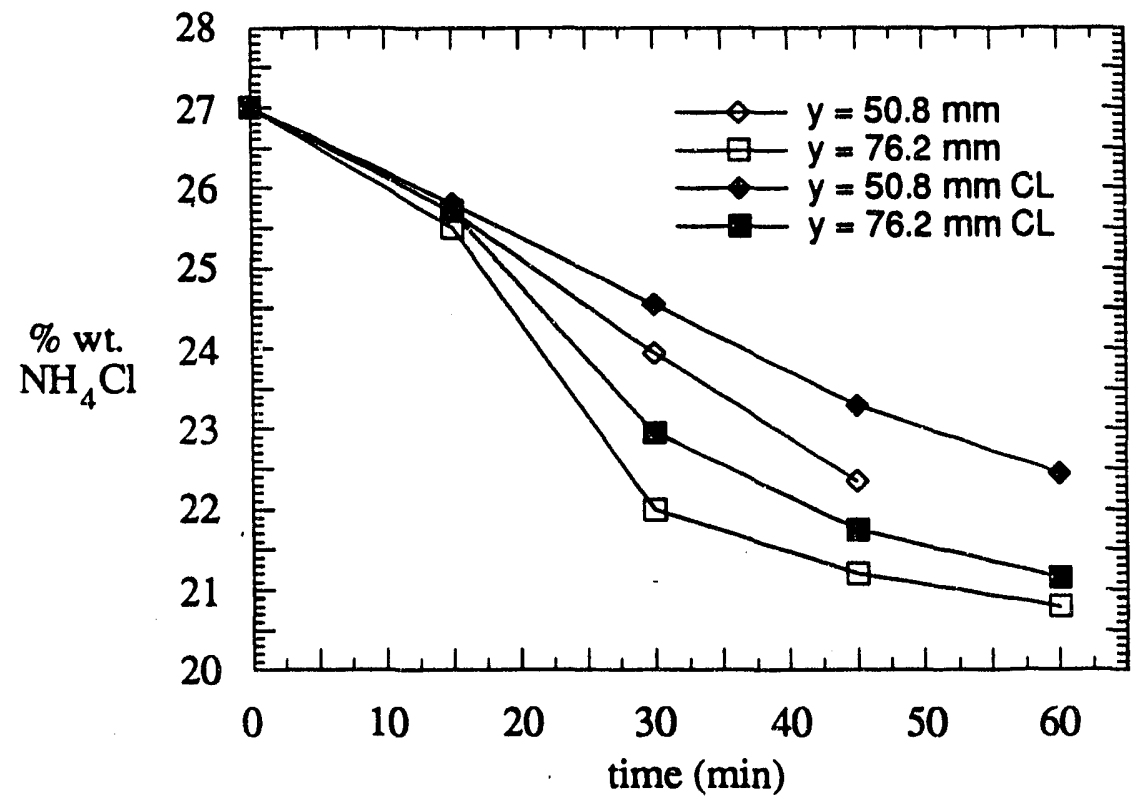

Figure 4.23 Liquid composition measurements at $x=25.4 \mathrm{~mm}$ (open symbols) and at the centerline (closed symbols). 


\section{CHAPTER 5 - EXPERIMENTAL RESULTS OF SOLIDIFICATION DURING ROCKING}

\subsection{Introduction}

A number of solidification experiments were performed under the influence of an oscillatory rocking motion. Both test sections I and II were rocked primarily at two cycles per minute with varying amplitudes, and Table 5.1 shows the corresponding experimental operating conditions. In most cases, a particular experiment was performed several times, the findings of which have again been integrated into the present discussion. Flow visualization studies using the shadowgraph technique were undertaken for the extreme rocking conditions, thereby offering insight into the effects of rocking on solidification. In taking the photographs, the camera rotated with the test section, thereby fixing the point of reference of each exposure. In order to determine the direction of gravity with respect to the test section, four plumb lines were placed in the column of light so as to cast thin, vertical shadows on the shadowgraph material. When starting a rocking experiment, the test section was set into motion at least one minute before the application of coolant to the heat exchangers. This procedure ensured that well established flow patterns were present in the melt as crystals first nucleated. Although this initial condition was imposed to match the initial condition used in the numerica! simulation (Chapter 6), it is suspected that little difference in growth morphology would result if chilling and rocking were initiated concurrently.

If a box containing an inviscid fluid were impulsively set into a rotational motion, the enclosed fluid, rather than rotating with the box, would remain oriented in the direction of an inertial reference frame. In other words, although stretching and 
Table 5.1 Experimental conditions for static and rocking experiments.

\begin{tabular}{|c|c|c|c|c|c|c|}
\hline Experiment & Conditions & $\begin{array}{c}\mathrm{T}_{\text {wall }} \\
\left({ }^{\circ} \mathrm{C}\right)\end{array}$ & $\begin{array}{c}\mathrm{T}_{\text {init }} \\
\left({ }^{\circ} \mathrm{C}\right)\end{array}$ & $\begin{array}{c}\text { Flow } \\
\text { Viz. }\end{array}$ & $\begin{array}{c}\text { Freq. } \\
(\mathbf{c p m})\end{array}$ & $\begin{array}{c}\text { Amplitude } \\
(\text { deg. })\end{array}$ \\
\hline UDS 1 & Static & -30 & 25 & $\times$ & - & - \\
\hline UDS 1R & Rocking & -30 & 25 & $\times$ & 2 & $45^{\circ}$ \\
\hline UDS 2R & Rocking & -30 & 25 & - & 2 & $30^{\circ}$ \\
\hline UDS 3R & Rocking & -30 & 25 & - & 2 & $15^{\circ}$ \\
\hline UDS 4R & Rocking & -30 & 25 & - & 1 & $45^{\circ}$ \\
\hline & & & & & & \\
\hline MDS 1 & Static & -30 & 25 & $\times$ & - & - \\
\hline MDS 1R & Rocking & -30 & 25 & $\times$ & 2 & $45^{\circ}$ \\
\hline MDS 2R & Rocking & -30 & 25 & - & 2 & $30^{\circ}$ \\
\hline MDS 3R & Rocking & -30 & 25 & - & 2 & $15^{\circ}$ \\
\hline
\end{tabular}

UDS - Unidirectional Solidification

MDS - Side and Bottom Wall Chilling 
deforming to match the shape of the rotating box, the fluid body itself would never rotate. However, to an observer seated on the rotating box (i.e. moving reference frame), the enclosed fluid would appear to rotate in a direction opposite to the actual motion of the box. If the box were rotated counterclockwise at one revolution per minute, the observer seated on the box would perceive the enclosed fluid as rotating clockwise at one revolution per minute. With relatively low viscosity, an aqueous saline solution contained in a rotating box would exhibit similar behavior.

To an observer seated on a rocking box, an enclosed solution of $\mathrm{NH}_{4} \mathrm{Cl}-\mathrm{H}_{2} \mathrm{O}$ would appear to rotate in the opposite direction from the imposed rocking. However, angular momentum from the box would be transferred to the fluid through viscous boundary layers which would develop on the enclosure walls, thereby complicating the flow and mixing the contained fluid. The flow characteristics of the rotating box have been numerically simulated, and the results are presented in Appendix B.

\subsection{Unidirectional Solidification}

\subsubsection{Qualitative Observations}

The photographic record included herein was taken for the case UDS $1 R$, in which the rocking rate was two cycles per minute and the rocking amplitude was $45^{\circ}$. As in the static case, crystals began to form on the heat exchanger wail within 15 seconds of applying coolant to the cold plate. The dendritic crystals grew rapidly and immediately began to reject water-rich fluid which rose, thereby inducing salt fingers. However, the rocking effect quickly asserted itself by sweeping the nascent salt fingers away from the liquidus interface (Fig. 5.1a). The first fingers that formed were quickly mixed into the melt so that after approximately two minutes of solidification, few salt fingers existed in the bulk fluid (Fig. 5.1b). Even after approximately seven minutes, Figure 5.1d shows that salt fingering in the melt, although present, was weak with small solutal gradients. 


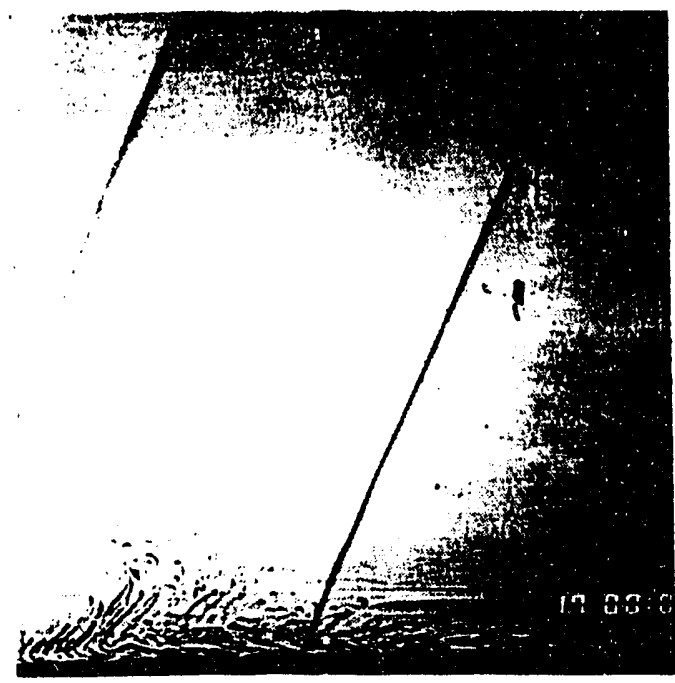

(a)

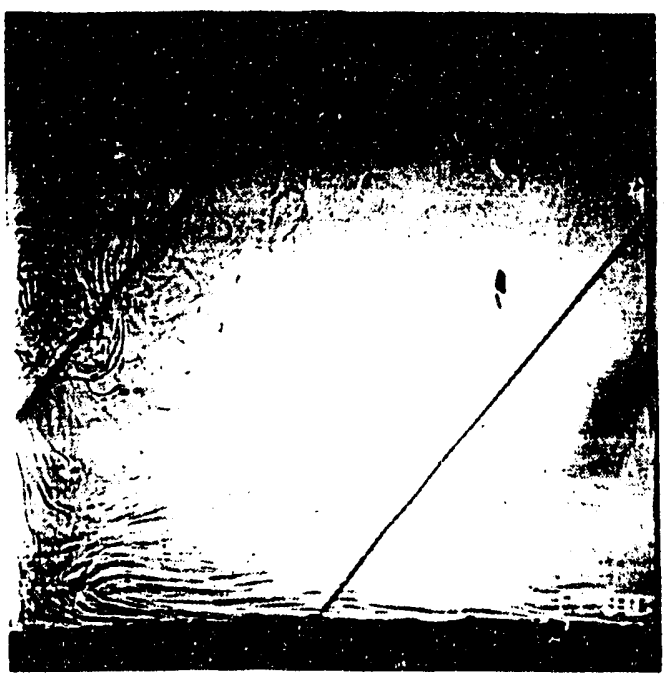

(c)

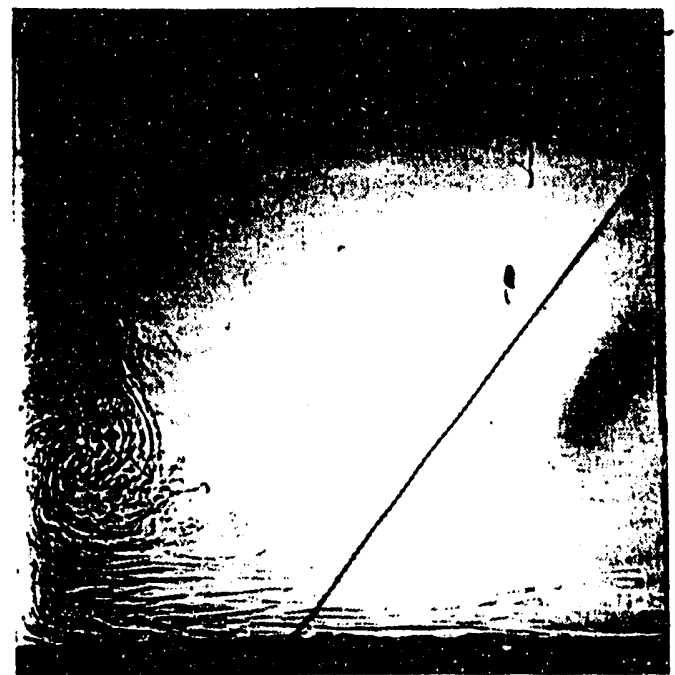

(b)

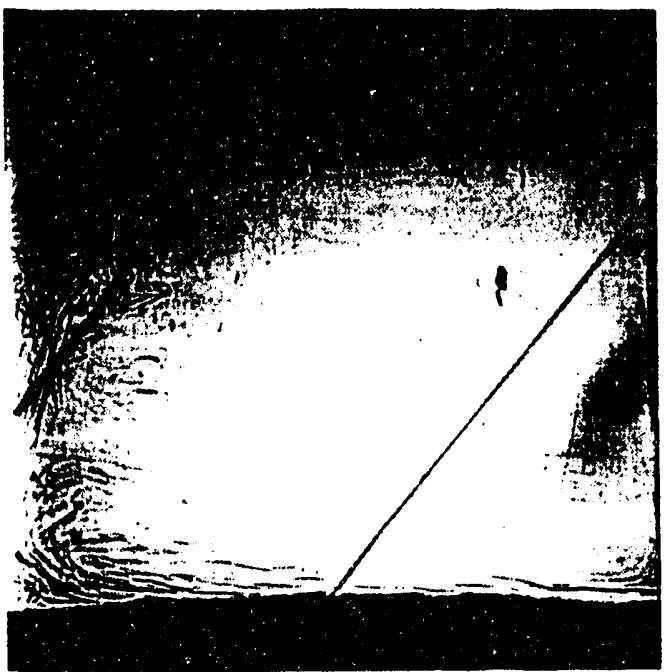

(d)

Figure 5.1 Shadowgraph images of early solidification for case UDS 1R: (a) 1 $\left(\theta=22^{\circ}\right)$, (b) $2\left(\theta=36^{\circ}\right)$, (c) $5\left(\theta=38^{\circ}\right)$, and (d) $7 \mathrm{~min}\left(\theta=38^{\circ}\right)$. 
The absence of strong, distinct salt fingers, like the kind seen during static solidification (Fig. 4.4), was testament to the efficient mixing by which the rocking motion stirred the melt. The crystalline growth front remained generally planar in the first stages of solidification, but a slight convex shape began to develop at seven minutes (Fig. 5.1d). It should be noted that in all shadowgraph rocking images, the time listed in the figure caption in only accurate to one minute intervals. Also, because the rocking rates are not exact, no correlation between the angular position of the cavity and the precise time can be made.

Convective motions within the melt were generated primarily by thermal and solutal buoyancy, and to a lesser extent by inertial effects arising from the rocking. A test section near or at its maximum tilt angle would produce free convection patterns in which chilled fluid near the liquidus interface cascaded downward, efficiently sweeping away solutal perturbations which would ordinarily lead to channeling. Within the mushy region, flow characteristics appeared to be dependent on location. At and just below the liquidus interface, crystal growth rejected water-rich fluid into the melt thus creating double-diffusive salt fingering. These fingers were especially visible when the test section was near the vertical position and thermal sweeping of the liquidus was minimized (not shown). However, flow deeper within the mushy region was significantly different. For example, when the test section was tilted to the left, a slurry developed just above the growth front at the far right end of the test section. This slurry, similar to that associated with side wall chilling, indicated thai water-rich fluid traversed the crystalline structure, perpendicular to the direction of crystal growth, fracturing dendrites and ejecting them into the melt at the right end of the grow ih front.

Lriven by physical mechanisms similar to those found in a side wall mushy region (see section 4.3), A-segregate type channels and double-diffusive convection cells would likely ensue if the UDS mold were to remain in its $45^{\circ}$ position. However, the 
rocking motion quickly changed convective conditions within the cast. As the test section rocked back toward the vertical position, inertial effects opposed those of thermally driven convection across the liquidus interface, permitting salt fingers to rise into the melt region. However, as the mold moved through the vertical position and toward the opposite extremum, the fingers were again sheared away. The period of rocking from vertical to the extreme amplitude was characterized by increasingly robust thermal convection, augmented by the existing inertial flow, which suppressed the sait fingers. Therefore, it is this alternating convective sweep of the incipient salt fingers which discourages any one finger from strengthening and forming a well defined, vertical channel. Although taken much later in the experiment, Figure 5.6 shows the characteristic fluid motion associated with rotation of the test section from the vertical position to its maximum amplitude.

The strong thermal boundary layers which formed at the liquidus front can clearly be seen in Figure 5.1c. Based on visual observations, maximum velocities in the boundary layers were estimated to be on the order of 1.5 to $2.0 \mathrm{~mm} / \mathrm{s}$. Acting as flow tracers, rising salt fingers were stretched into horizontal streaks, thus revealing the presence of the layers. Within the layers, eddies generated by thermal and solutal buoyancy produced random fluctuations in temperature and composition in a fashion similar to turbulence. However, no small scale vorticity, produced by instabilities arising from shear forces in the flow, was observed. Herein lies the distinction between the random fluctuations associated with the thermal boundary layer during soildification and classic, shear-induced turbulence arising from high Reynolds number flow. In as much as salt fingers can be considered turbulent, so too can the falling thermal boundary layers, but this turbulence was not generated by small scale, shear induced vorticity.

At this time, it should be noted that, contrary to design objectives, flow conditions in both test sections were influenced by boundary layers which developed on the front 
and back Lexan viewing plates during an oscillatory sweep and hence, were highly threedimensional. Not surprising in retrospect, the boundary layers, caused by the plates pivoting across the contained fluid, led to counter-rotating vortices which swept the liquidus front in the z-direction (Fig. 5.2). The vortices were first observed as they entrained crystals from the slurry zone just above the liquidus interface, and were generated entirely by inertial effects. However, owing to the characteristics of the test section and rocking, they were difficult to capture photographically. Although difficult to discern their exact nature, it was observed that the vortices tended to rotate primarily in the $y-z$ plane, and they acted to both augment and oppose solutal buoyancy during a cycle.

Although it was not recorded photographically, a fairly dense slurry region was also present in the rocking experiments. Not as vigorous as slurry regions formed during static side wall chilling experiments, the slurry formed during the UDS $1 \mathrm{R}$ case was nevertheless considerably more robust than those formed during static UDS cases. This behavior was most likely due to stronger interdendritic convective flows. The more robust slurry appeared to consist of dendrite fragments whicil originated from within the mushy region, rather than of fragments which were sheared from dendrite tips at the liquidus interface. It is not altogether clear why secondary dendrite arms within the mushy region would be weaker than those near the melt. It may be that smaller, more fragile crystals formed within the mush and hence were easily broken by interdendritic cross-flow. A more probable explanation seems to point to the breakdown of secondary dendritic crystals by remelting resulting from salt enrichment and/or the release of latent heat during crystal nucleation (i.e. recalescence). Other investigators have observed and photographically recorded such morphological behavior in a dendritic array of $\mathrm{NH}_{4} \mathrm{Cl}$ $\mathrm{H}_{2} \mathrm{O}$ (Neilson and Incropera, 1991). In any case, the free floating crystals eventually descended back to the liquidus interface, promoting equiaxed crystal growth. 


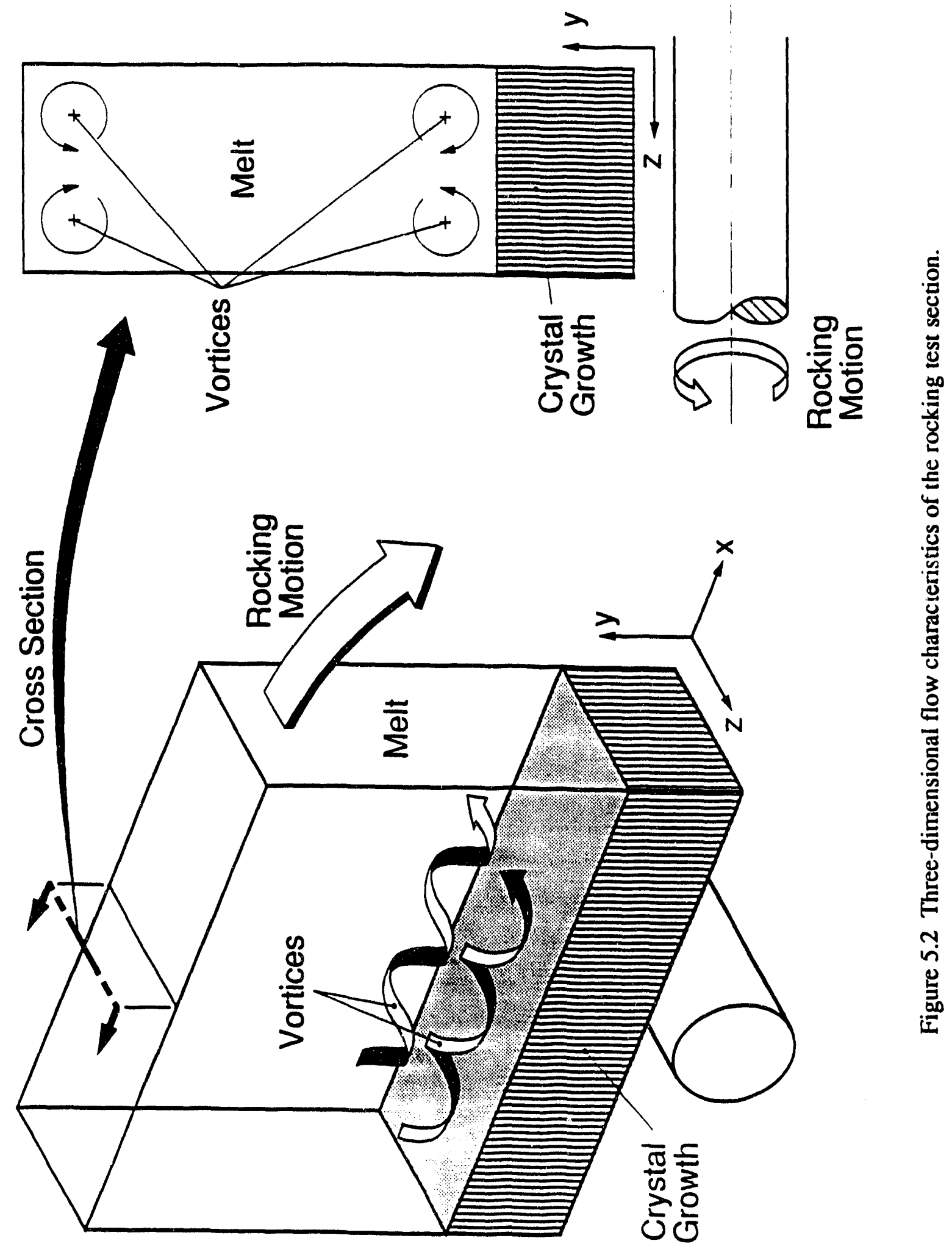


From 15 to 30 minutes, the thermal boundary layers weakened and solutal buoyancy effects became more prevalent. However, the rocking motion was still effective in maintaining a well mixed melt and in suppressing channels. Only a small number of relatively weak salt fingers issued from the liquidus interface at 15 minutes, and those fingers were quickly swept into the melt (Fig. 5.3a). In fact, the sweeping motion shown in Figure 5.3a comes not from buoyancy, but from inertial forces. The photograph was taken as the test section rocked from the left to the vertical, thereby generating inertial forces which drove the fluid in a counterclockwise direction. At approximately 20 minutes, Figure 5.3b shows that, tilted to the far left, strong boundary layers continued to sweep the liquidus front. Also shown is the entrainment and exiting of fluid to and from the boundary layer. At the top of the boundary layer (lower right of the cavity in Figure 5.3b), fluid from the melt flows downward along the end wall impinging on the mushy region and turning sharply to the left. Once in the boundary layer, flow is generally parallel as it sweeps across the test section eventually impinging on the left end wall. Turning away from the mushy region, the fluid does not flow up the end wall, but rather, owing to solutal buoyancy gained by sweeping the mushy region, separates from the end wall moving to the center of the melt. At approximately 30 minutes, similar flow conditions exist, but, as seen when the test section rocks from the left to the vertical (Fig. 5.3c), the salt fingers had gained strength, gathered into distinct plumes and were issuing into the melt at several sites along the liquidus interface.

The crystal structure which developed along the liquidus interface was different from the grain-like structure formed under static conditions. Namely, each individual primary dendrite, though columnar, was randomly oriented in a unique direction relative to its neighbors when viewed from above. Therefore, each dendrite was itself a single crystal. In contrast, the crystal structure formed under static conditions consisted of large regions in which the dendrites were oriented in the same direction, thereby forming a 


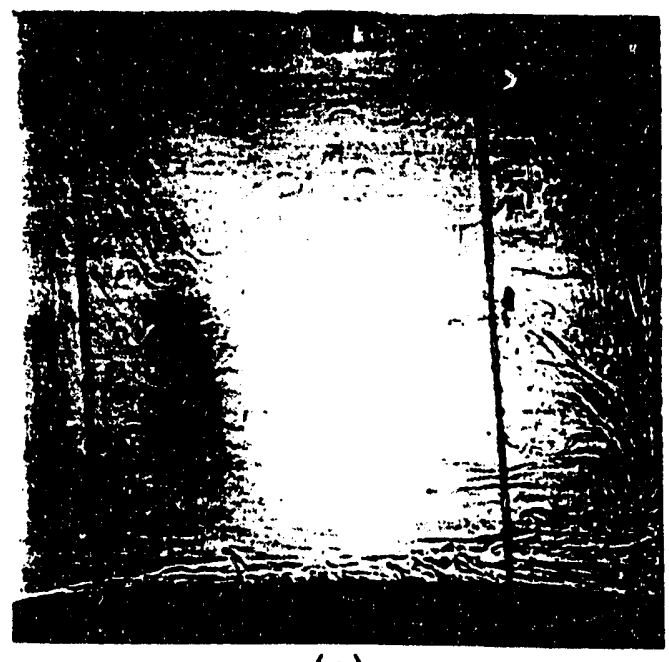

(a)

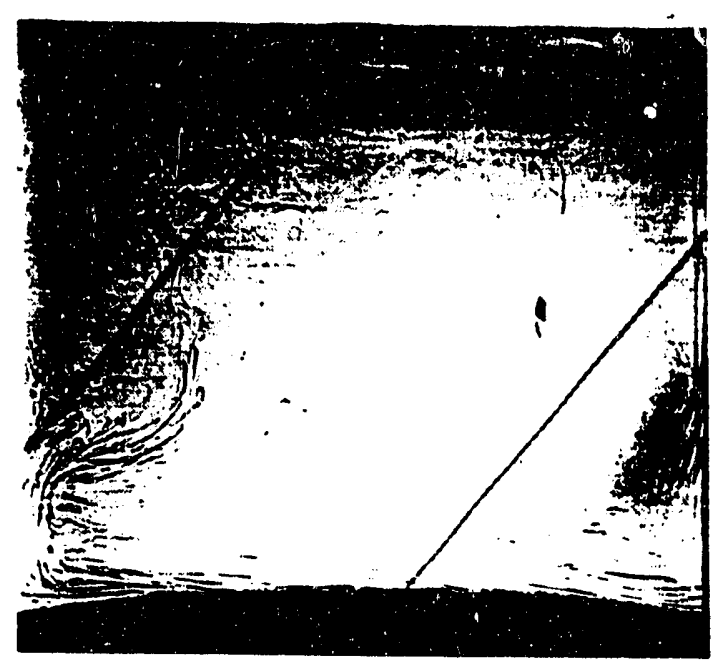

(b)

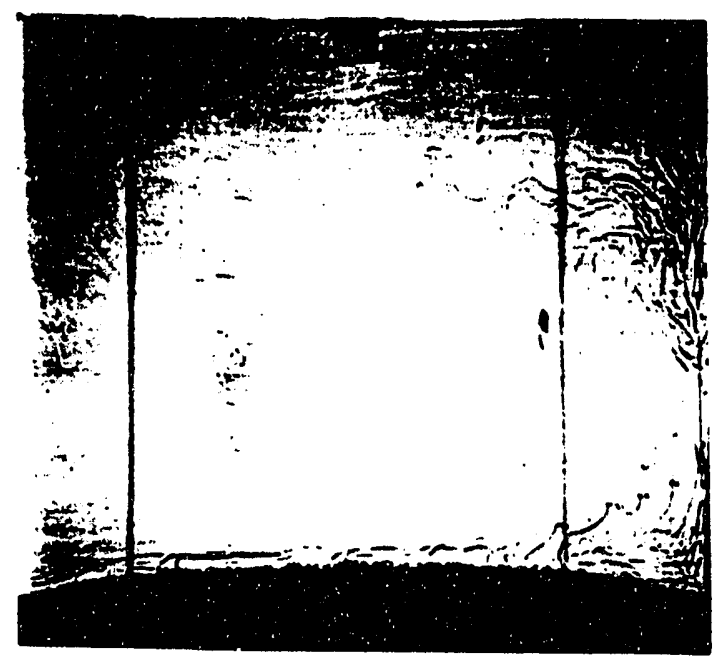

(c)

Figure 5.3 Shadowgraph images for case UDS $1 \mathrm{R}$ showing robust salt fingering and mixing: (a) $15\left(\theta=-4^{\circ}\right)$, (b) $20\left(\theta=38^{\circ}\right)$, and (c) $30 \min \left(\theta=0^{\circ}\right)$. 
single grain, which itself was a single crystal. In addition, the primary dendrites which formed under rocking conditions extended into the melt, creating a jagged liquidus interface. This seemingly random orientation of the primary dendrites was present in all rocking experiments and was most likely caused by the robust slurry which formed in the early stages of solidification. The large number of free floating crystals which fell onto the mushy region disrupted the developing grains and promoted the growth of new independent crystals from many sites along the growth front, thereby creating a somewhat disorganized, quasi-columnar crystalline structure.

At approximately 45 minutes, small holes began to form in the interstices of some primary dendrites. These holes were very similar to those formed by incipient channels in the static UDS case; however, they did not quickly enlarge to form full channels. They did, however, act as pathways for interdendritic fluid to escape as plumes into the melt (Fig. 5.4). Like the salt fingers that preceded them, these weak plumes were deflected and sheared away by the ubiquitous thermal and inertial boundary layers. Figure 5.4 shows the flow characteristics as the test section rocked from $+13^{\circ}$ to $-38^{\circ}$. Approaching the vertical position from the right, an inertially induced clockwise fluid rotation pushed the rising plumes to the left (Fig. 5.4a). Continuing to rock over to the left, thermal boundary layers developed thereby augmenting inertial convection and robustly sweeping the liquidus interface (Fig. 5.4b). The rocking motion therefore effectively prevented incipient plumes from strengthening and supporting the development of mature segregate channels. However, illustrating the strong buoyant potential contained within the mushy region and consistent with findings of previous investigators (Sample and Hellawell, 1982), if the rocking were stopped during solidification, channels and strong plumes would quickly form. Interestingly, those channels could then be resuppressed by resuming the rocking motion. 


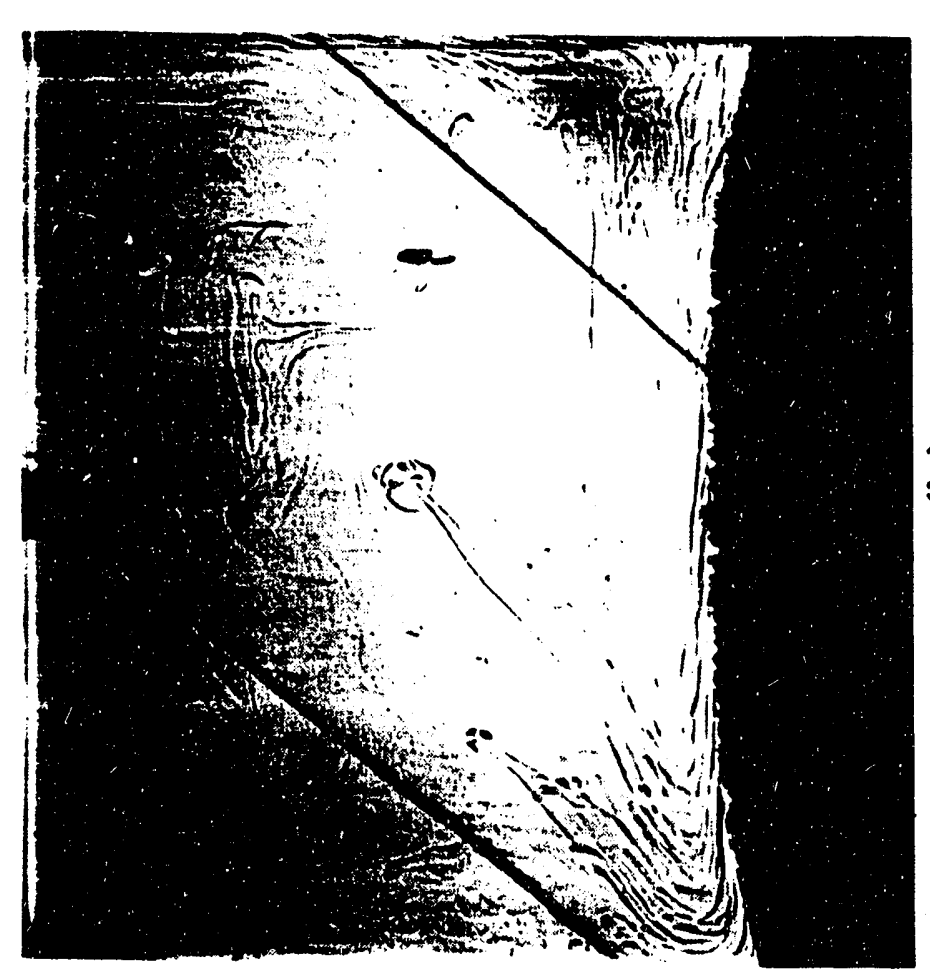

峞

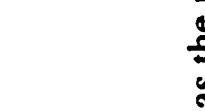

远

.

을

a

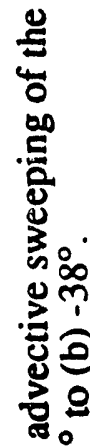

일ำ

产吉

कै

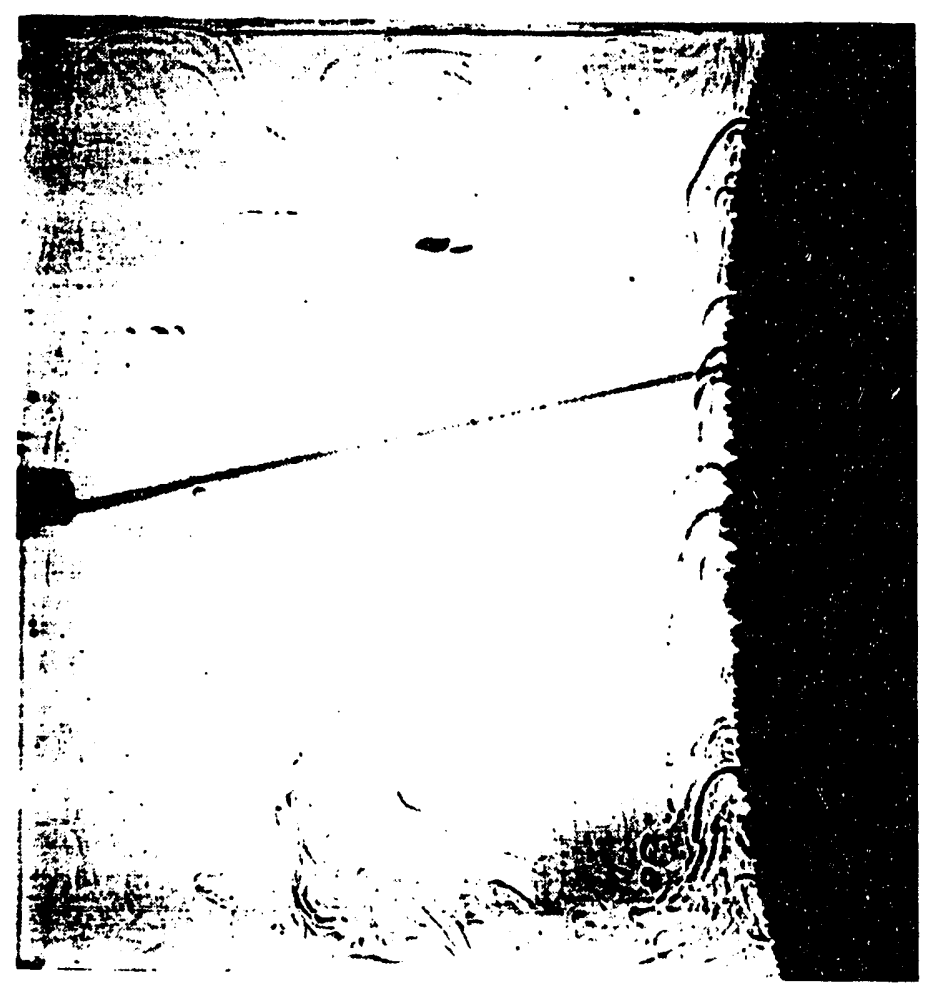

今 
Beyond one hour, solidification continued slowly as a handful of the small channels competed for the dwindling supply of interdendritic fluid. Figure 5.5 shows conditions at seltcited times beyond one hour. Each photograph was taken as the test section rocked from the left to the vertical position, thereby producing a counterclockwise, inertially induced flow rotation in the melt. It is important to note that individual plumes do not survive for an extended period of time. For example, the relatively robust plume to the left of the cavity at 60 minutes (Fig. 5.5a) had completely disappeared at 75 minutes (Fig. 5.5b). In fact, due to advective washing at the liquidus interface produced by the rocking, channels abruptly opened and closed over the later stages of the experiment, and no single channel ever fully developed. This observation implied that localized freckling caused by mature channels should be eliminated by the rocking motion. However, relatively strong plumes did emanate from those channels that were open. The strong plume present at 60 minutes, though stretched by the fluid motion, was not easily mixed with the melt, and instead broke into a number of rising buoyant structures (Fig. 5.5a). Figure 5.5c shows a well-defined, free-floating plume, sheared away at its base, as it advected through the melt.

During the later stages of the experiment, from three to four channels within the mushy region became nearly as large in diameter as the typical channel formed under static conditions. However, these channels were morphologically different from those formed under static conditions. Due to entrainment and localized cooling, channels formed under static conditions were encircled by a thick crystalline sheath of relatively low permeability. Also, the sheath extended from the base of the hole to above the liquidus interface forming volcano-like structures around the mouths of the channels. In contrast, under rocking conditions, elongated areas of secondary dendrites melted away from the crystalline structure leaving behind voids or fissures in the cadre. These fissures were generally about $5 \mathrm{~mm}$ long and, forming between ridges of the primary dendrites, 


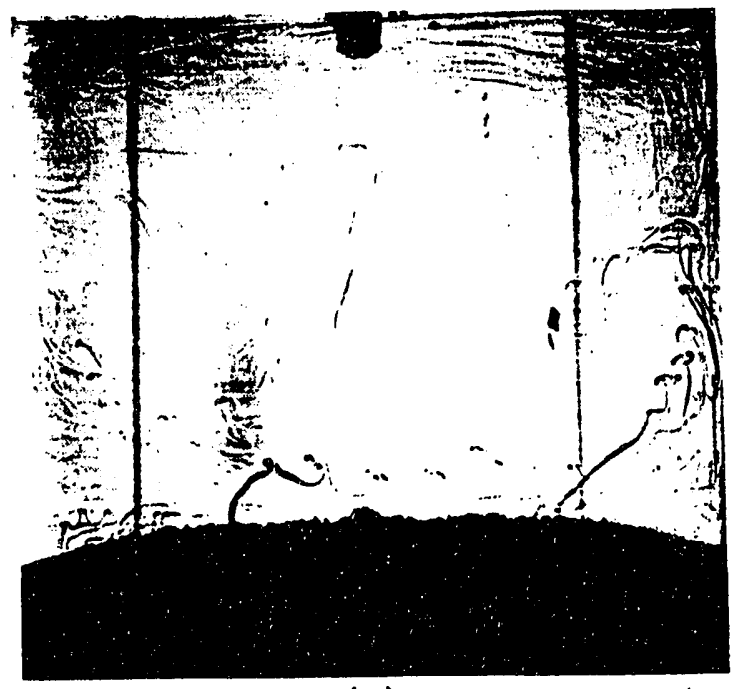

(a)

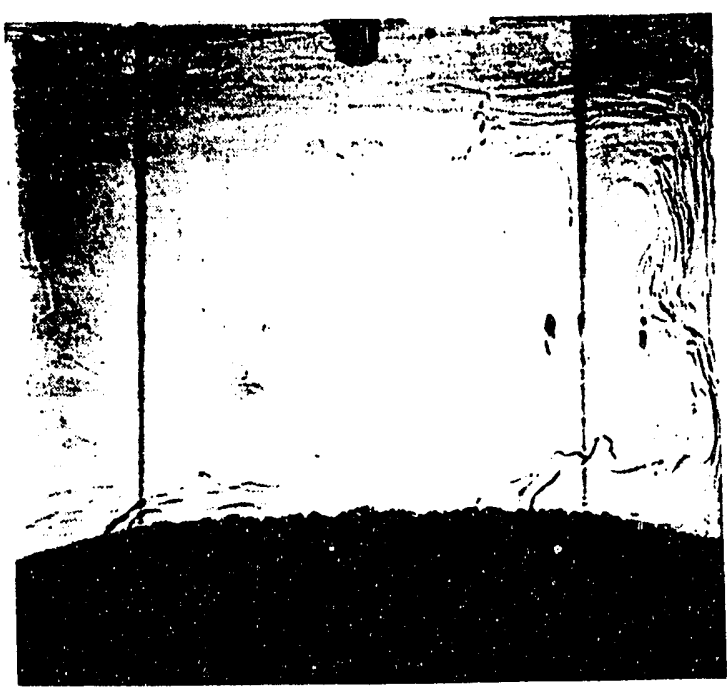

(b)

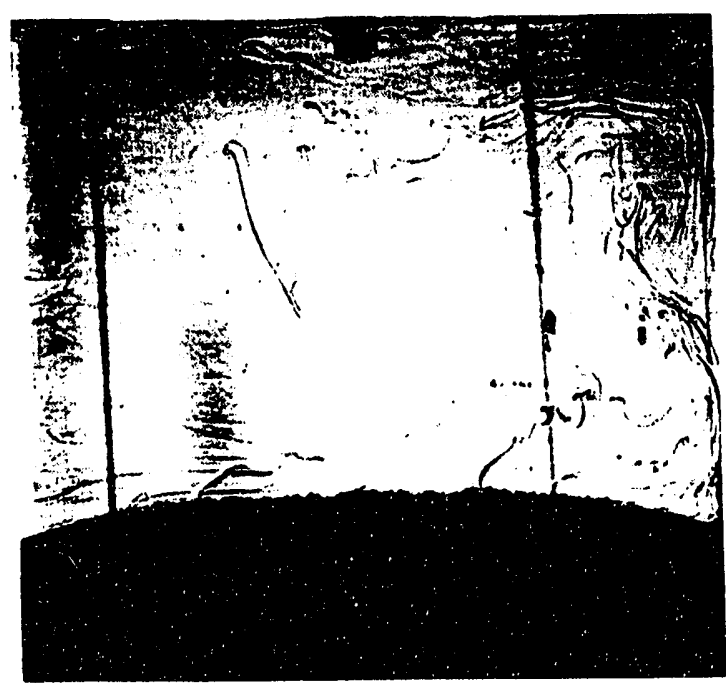

(c)

Figure 5.5 Shadowgraph images of later stages of solidification for experiment: UDS IR (a) $60\left(\theta=0^{\circ}\right)$, (b) $75\left(\theta=0^{\circ}\right)$, (c) $92 \min \left(\theta=-3^{\circ}\right)$. 
they were as wide as the primary dendrite arm spacing $(\sim 1.5 \mathrm{~mm})$. Early in their development, water-rich fluid would issue from the entire fissures, and eventually, distinct channels would develop from the fissures. However, these channels were encircled by a relatively ill-defined crystalline sheath with a permeability similar to the surrounding mushy region, and no volcano-like structures extended above the liquidus interface. In addition, the holes were not necessarily vertical, as they appeared to draw large amounts of interdendritic fluid from the accompanying fissures.

Figure 5.6 shows flow conditions over the course of 7.5 seconds at approximately 105 minutes into the experiment. The three photographs illustrate how developing thermal and inertial boundary layers act to shear and mix plumes emanating from the mushy region. As the test section rocks from vertical to the far left, convection patterns act to mix the melt, and more importantly, buoyant plumes are clearly washed away by the falling thermal boundary layer.

At two hours into the experiment, weak plumes were ejected from four small holes into the melt. In general, the melt was well mixed and free of any double-diffusive convection cells. The liquidus front was convex, with the liquidus and solidus interfaces located 42 and $19 \mathrm{~mm}$, respectively, from the cold wall at the center and 35 and $15 \mathrm{~mm}$ from the cold wail at the ends (Fig. 5.7). In comparison, the liquidus and solidus fronts in the UDS static case were located 40 and $17 \mathrm{~mm}$, respectively, from the cold wall at the end of two hours. The convex growth front was primarily due to warm fluid from the melt impinging onto the liquidus front during the course of an experiment. The rocking acted to suppress crystal growth at the ends by impinging warmer fluid from the melt onto the mushy region. However, the total time expected to completely solidify a cast is believed to be less under rocking conditions, because the additional convection generated by the rocking motion more efficiently chills the melt than solidification under static conditions. 


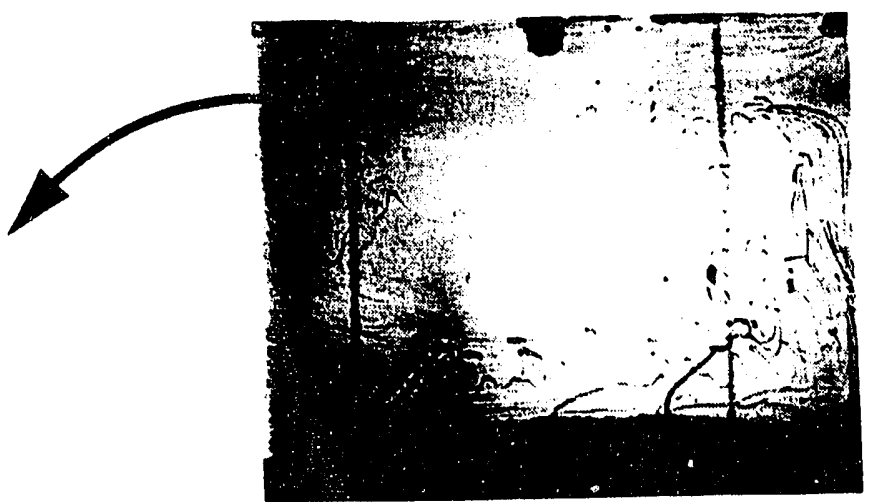

(a)

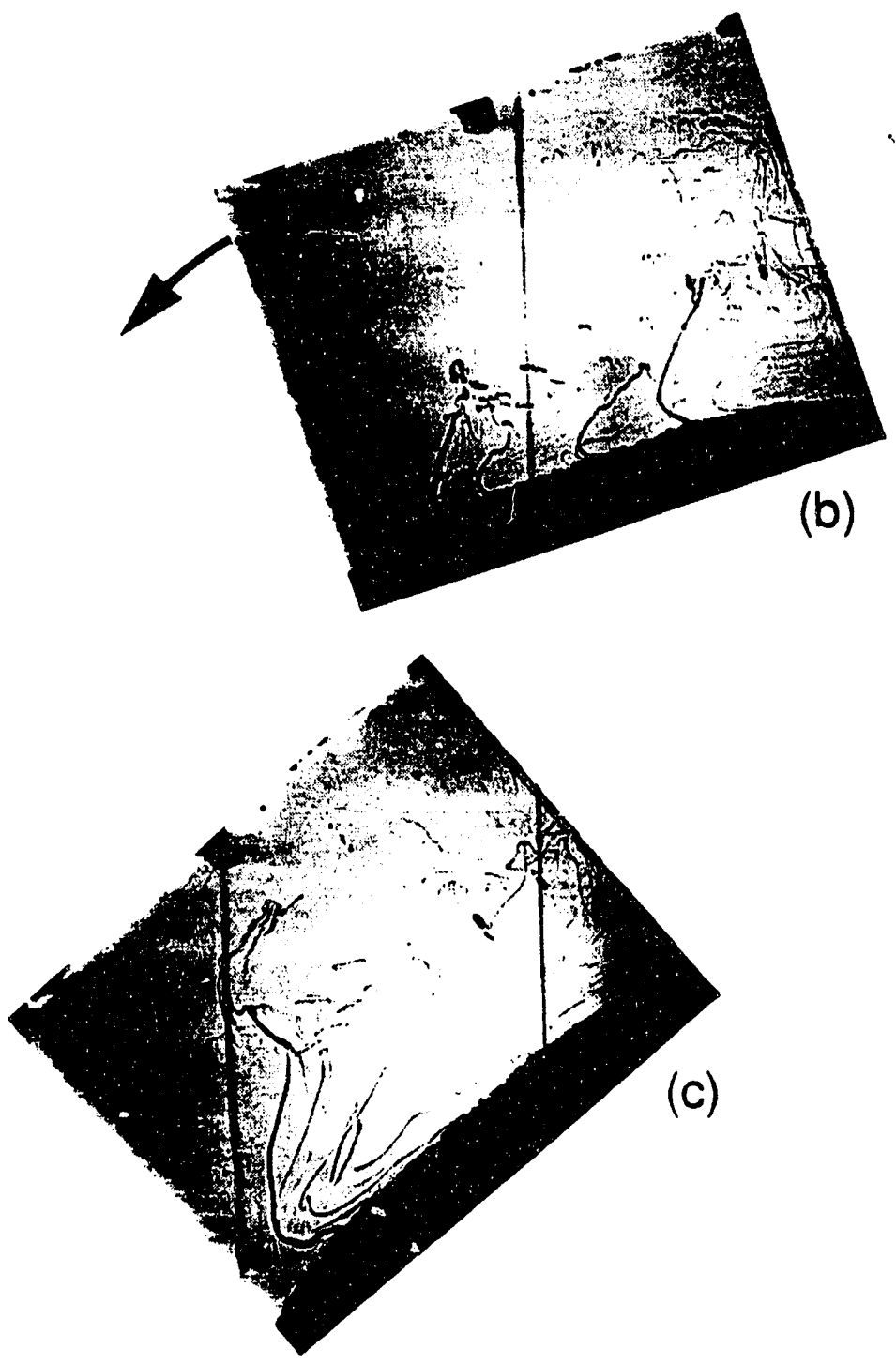

Figure 5.6 Sequence of images over $7.5 \mathrm{~s}$ interval at $105 \mathrm{~min}$ for case UDS 1R: test section rocks from (a) the vertical position, to (b) $18^{\circ}$, to (c) $40^{\circ}$. 


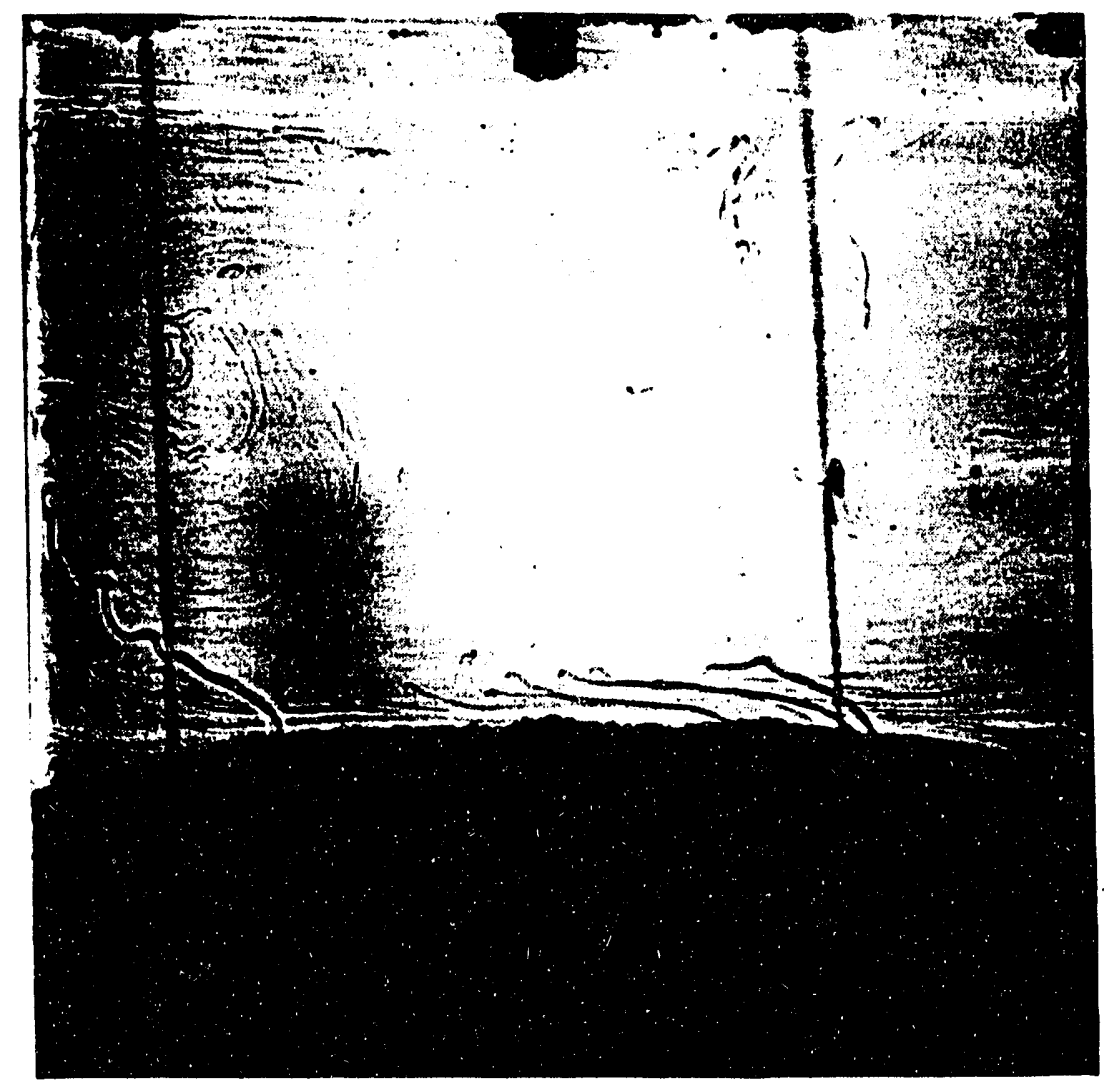

Figure 5.7 Final shadowgraph image for case UDS 1R at $120 \min \left(\theta=-3^{\circ}\right)$. 
The quasi-columnar structure of the crystals grown under rocking conditions can clearly be seen in Figure 5.8a. Meeting the objectives of UDS, the dendrites were generally oriented in the vertical direction, leading to greater strength and directional performance. The structure is similar to static casts if viewed from the side, but if viewed from the top, striking differences between rocking and static crystal morphology become apparent (Fig. 5.8b and c). As discussed before, individual primary dendrite orientation was random under rocking conditions. However, owing to the induced fluid sweeping from the left to right and right to left for the conditions of in Figure 5.8b, ridges of primary dendrites would develop across the width of the test section. Figure 5.8b shows these ridges extending from the top to the bottom of the image. Holes and channels which were relatively small can also be seen in the figure, and one well defined channel formed near the bottom edge, about two-thirds the distance from the right end. Comparing those channels and holes with the channels formed under static conditions (Fig. 5.8c), differences in the crystalline sheath and permeability are clearly seen. Whereas the channel in the upper left of Figure 5.8c has a thick, overgrown dendritic sheath encircling it, even the most well defined channel of Figure 5.8b shows little evidence of a thick surrounding sheath. However, away from the channels, the crystalline grain structure grown under static conditions is much more ordered than that grown under rocking conditions and may be structurally stronger.

\subsubsection{Quantitative Results}

As in the static UDS tests, cooling curves and species composition measurements were recorded for each rocking experiment mentioned in Table 5.1. Temperatures were recorded approximately every 15 seconds at predetermined sites within test section I (see section 2.4.2), and selected multiple experiments confirmed that the cooling curves were 


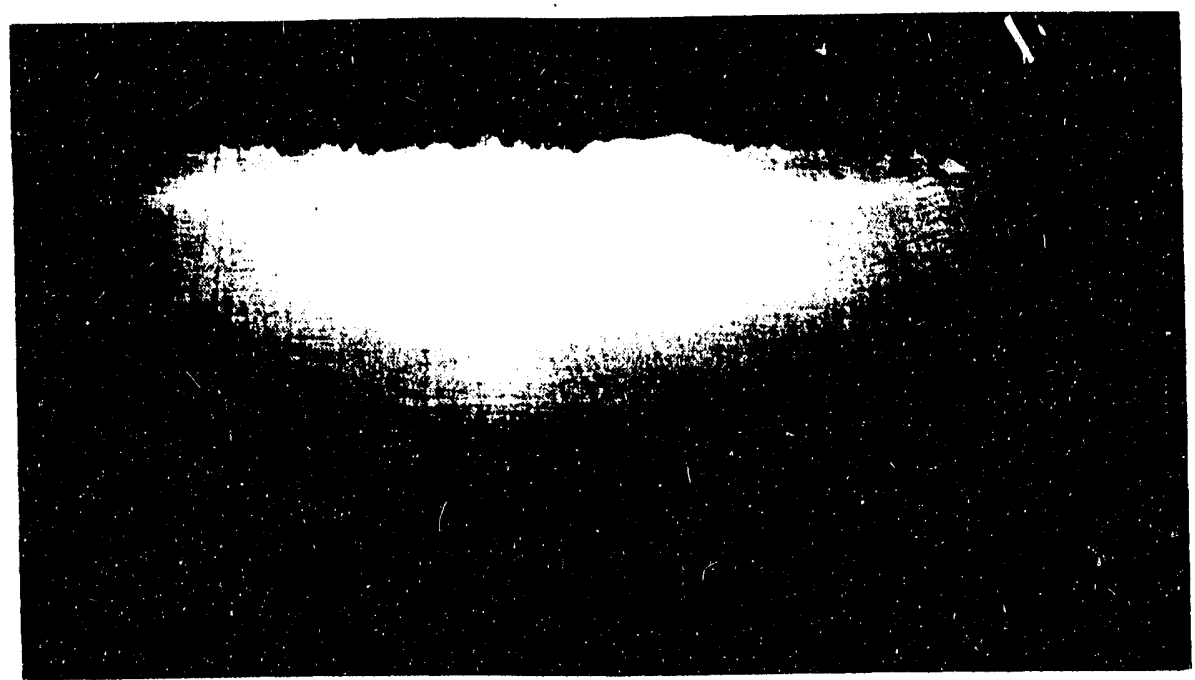

(a)

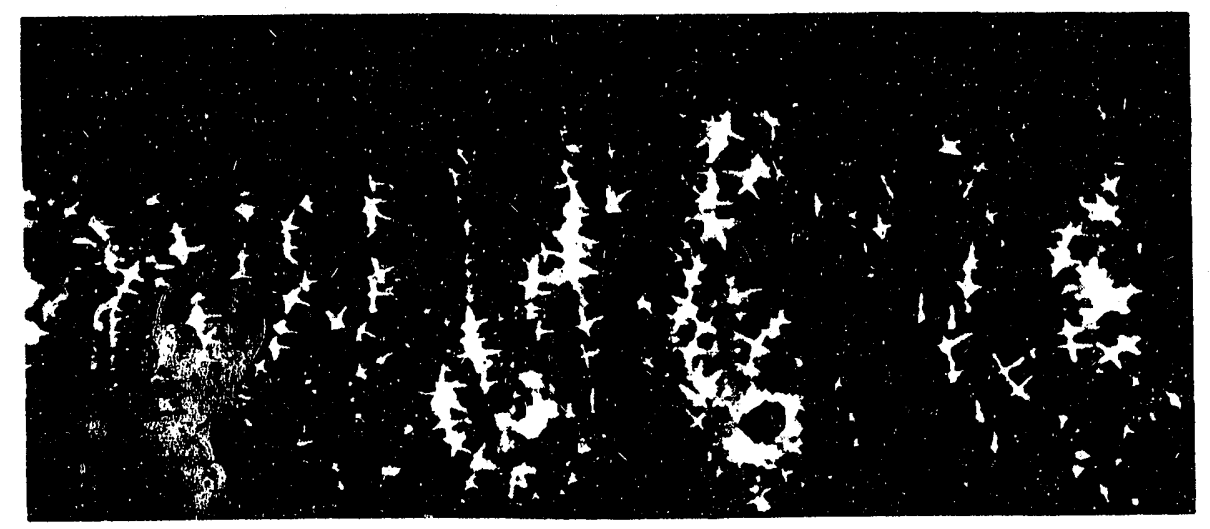

(b)

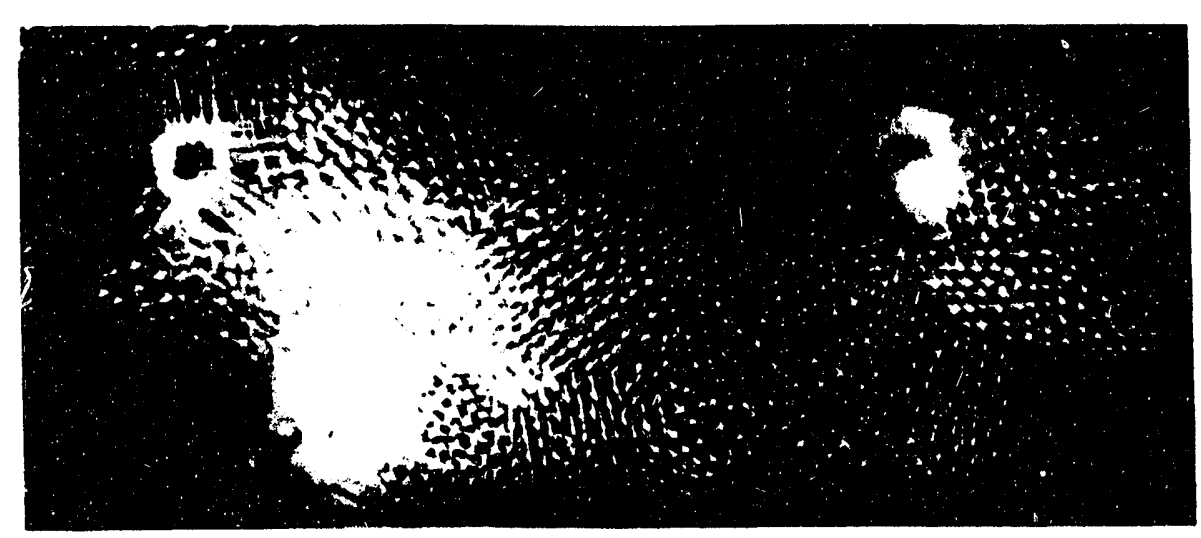

(c)

Figure 5.8 Mushy region morphology of case UDS $1 R$ as viewed from the (a) side and the (b) top. Also top view (c) of statically grown mushy region. 
repeatable. The most striking feature of the cooling curves for all rocking cases was the very vigorous oscillation that developed within the melt.

For the UDS $1 \mathrm{R}$ case, temperature fluctuations as large as $4^{\circ} \mathrm{C}$ developed in the melt (Fig. 5.9). These fluctuations were most severe just above the liquidus interface and in the early periods of an experiment when large thermal gradients preceded the growth front. When the test section moved to the vertical position, convection decreased allowing conduction to rapidly chill the liquid ahead of the mushy region. However, as the test section continued to rock to its maximum amplitude, strong thermally driven boundary layers swept this cold fluid away from the interface, replacing it with warm fluid from the melt. This intermittent chilling and rewarming process ahead of the growth front occurred four times each minute for rocking rates of two cycles per minute, and the resulting cooling curves reflected the cyclical nature of the rocking. Also, the temperature oscillations were largest just above the liquidus interface.

Figure 5.10 focuses on temperatures in the melt from 15 to 19 minutes when the lowest thermocouple location in the melt $(y=19.1 \mathrm{~mm})$ is about $6 \mathrm{~mm}$ above the liquidus interface. As mentioned before, temperature fluctuations just above the growth front are the largest. In fact, as the warm fluid from the melt is swept to the liquidus interface, the temperature at $y=19.1 \mathrm{~mm}$ actually rises above the temperature at $\mathrm{y}=25.4$ and $38.1 \mathrm{~mm}$. Also note that temperatures at the top of the melt tend to fluctuate in directions opposite to those of temperatures at the botiom of the meit. As warm fluid from the melt moves to the liquidus interface, cold fluid from the interface moves to the top of the cavity, creating temperature fluctuations at the top and bottom of the melt which tend to be oppositely phased. However, temperatures at the center of the melt $(y=50.8$ and 63.5 $\mathrm{mm}$ ) are relatively free of fluctuations, further indicating that there exists a general fluid rotation around the periphery of the melt and that the center is primarily quiescent. 


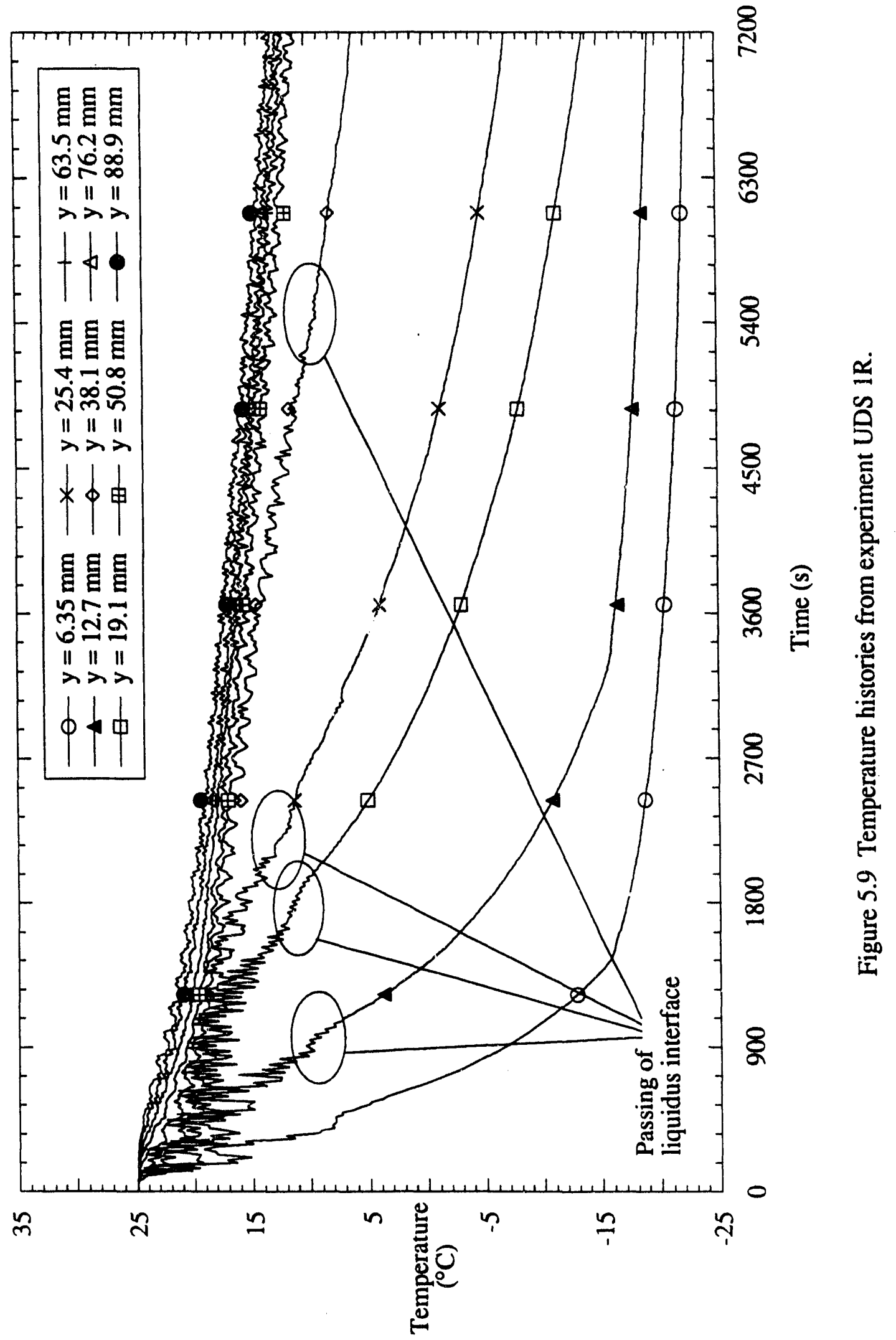




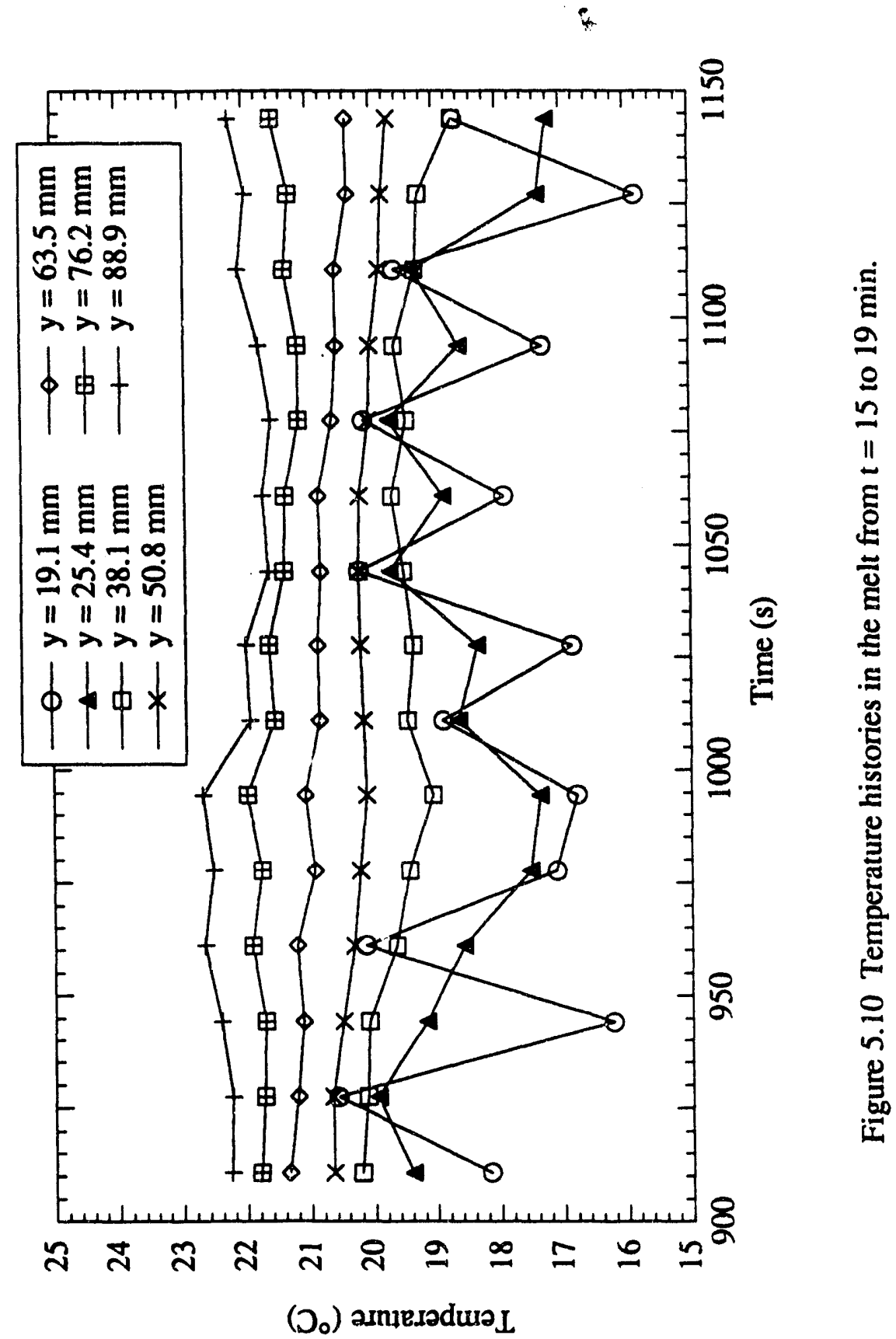


Although the cooling curves showed that, even with rocking, advection deep within the mhy region was effectively suppressed by D'Arcy damping, conditions at and just below the liquidus interface were noticeably affected by convective shear at the liquidus interface. This finding is important because channels develop from density perturbations at and just below the liquidus interface. Therefore, the rocking has a favorable influence at the liquidus interface by sweeping perturbations into the melt, resulting in the suppression of channeling. Ovirgrowth of a particular thermocouple location by the mushy region can generally be deduced from the cooling curves (Fig. 5.9) as that time when the temperature fluctuations at a particular location were suddenly damped. As time progressed, temperature fluctuations decreased with decr ing thermal gradients in the melt.

Within the temperature data presented in Figure 5.9, high frequency oscillations associated with the roching motion having a period of about 30 s are contained within slower oscillations having a period of about $180 \mathrm{~s}$. The longer oscillations are simply an artifact of the data sampling procedure. Because the sampling rate was on the same order of magnitude as the rocking frequency, the cooling curves do not provide a complete representation of actual transient betavior. That is, the temperature recorded at a particular time provided only one small measure of the oscillating temperature history and was as likely to record the mean of the oscillation as it was to record the peak or valley. Therefore, over the course of an experiment, the temperature sampling drifted in and out of phase with the temperature oscillations, creating the illusion that a long wave oscillation existed in the data. Such a phenomenon is often referred to as an alias in the signal processing literature. Although potentially misleading, the data are by no means incorrect, and important information can still be deduced from the curves.

As in the static UDS case, temperatures decreased steadily with time over the course of an experiment. However, unlike the static case, the melt was very well mixed, 
and temperatures at $y=50.8$ and $88.9 \mathrm{~mm}$, for example, varied by at most $3^{\circ} \mathrm{C}$ (Fig. 5.9). By contrast, the melt in the static experiments was highly stratified and temperatures varied by as much as $8^{\circ} \mathrm{C}$ (Fig. 4.13).

Figure 5.11 shows how temperatures at the upper three locations differed significantly between rocking and static conditions. From the beginning, temperatures with rocking fell more rapidly and conditions were better mixed than for the static case. Although rocking stratified fluid in the melt more quickly, with increasing time, thermal stratification in the static melt became more pronounced, as temperatures in the rocking melt became more uriform due to improved mixing.

The temperatus: data for experiment UDS $2 \mathrm{R}$ are shown in Figure 5.12, and although fundamentally similar to the cooling curves of UDS $1 R$, differences do exist. Although the rocking amplitude was smaller, temperature oscillations just above the liquidus interface were larger in the early stages of the experiment. For example, the temperature at $\mathrm{y}=12.7 \mathrm{~mm}$ varied by as much as $6^{\circ} \mathrm{C}$ at 450 seconds, before this location was enclosed by the mushy region. Similar vigorous fluctuations can be seen in other temperature profiles. Another interesting feature of the UDS 2 R cooling curves is the absence of higher frequency fluctuations in the melt temperatures toward the end of the experiment. However, as in the UDS $1 \mathrm{R}$ case, the melt is well mixed and nearly isothermal, with maximum temperature differences not exceeding $3^{\circ} \mathrm{C}$. Although rapid temperature fluctuations were recorded at the lower thermocouple locations early in the experiment, temperature histories in the melt generally showed only long scale oscillations. These long oscillations resulted from an alias in the data sampling, and short scale temperature fluctuations were present during the experiment. Although temperature histories recorded for the other experiments show some evidence of the short scale oscillation, it is not altogether clear why small fluctuations did not appear in the data of UDS $2 R$. A possible explanation is that the sampling rate and rocking rate for this 


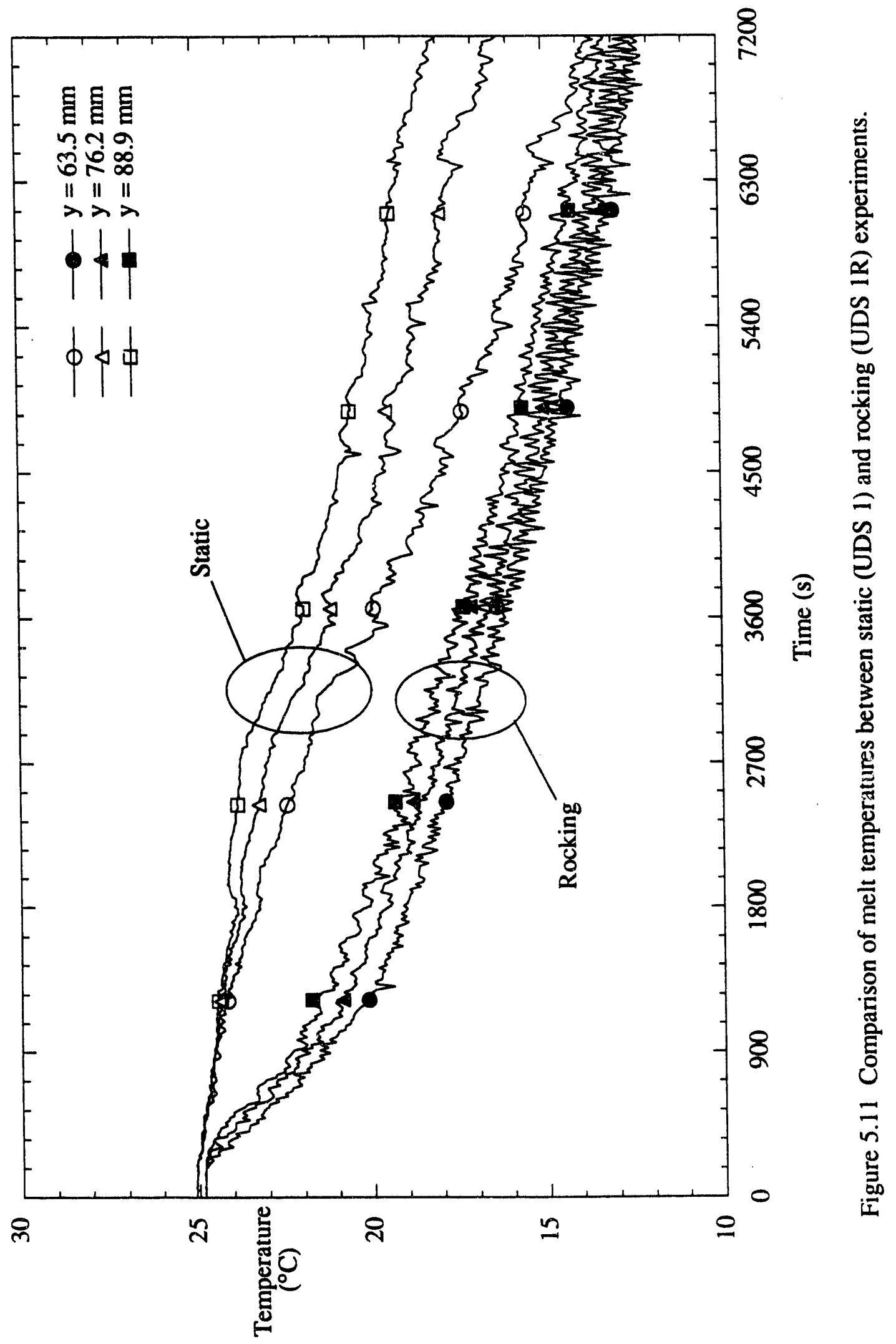




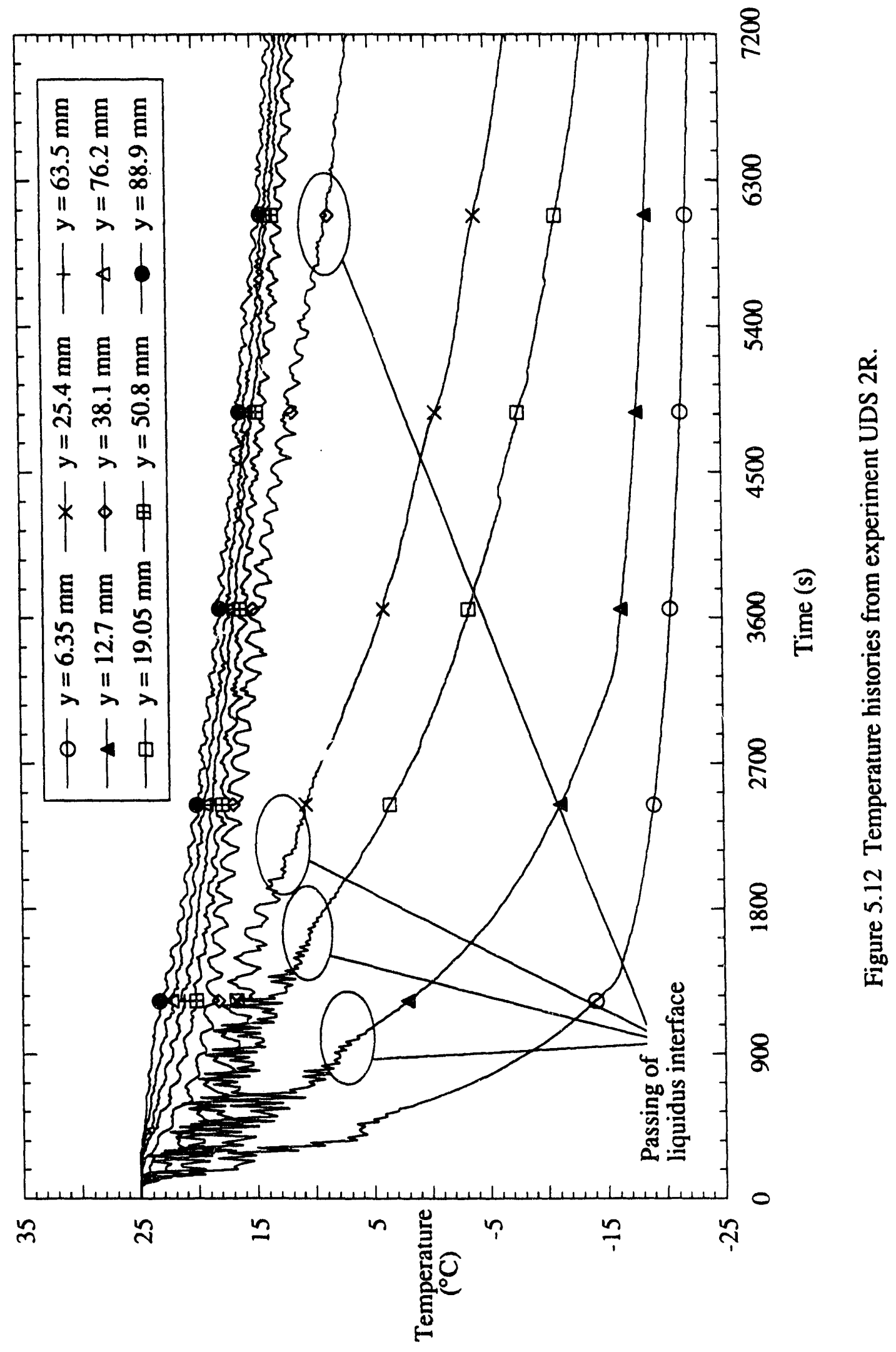


particular experiment were so closely matched that the small frequency waves never appeared.

When the rocking amplitude was reduced to $15^{\circ}$, the cooling curves began to resemble those associated with static solidification (Fig. 5.13). Temperature fluctuations at the liquidus interface were again very robust in the early stages of the experiment. However, these fluctuations did not penetrate far into the melt. Therefore, temperature fluctuations, in general, were not as severe, and a larger stratification, on the order of $4^{\circ} \mathrm{C}$, developed in the melt. The temperature stratification is based on the maximum temperature difference across the melt. As with other rocking experiments, thermal boundary layers developing on the liquidus interface induce a fluid circulation around the periphery of the melt and a nearly quiescent pool of fluid collects at the center of the melt. Figure 5.13 clearly shows this feature toward the end of the experiment, when the temperatures at $\mathrm{y}=50.8$ and $88.9 \mathrm{~mm}$ continued to oscillate while temperatures at $\mathrm{y}=$ 63.5 and $76.2 \mathrm{~mm}$ exhibited a nearly monotonic decay and became nearly isothermal.

The final rocking experiment, case UDS $4 R$, involved a rocking amplitude of $45^{\circ}$ and a rocking rate of one cycle per minute (Fig. 5.14). A surprising result is that, despite the reduced frequency, the cooling histories ahead of the growth front fluctuate more robustly than for any other case. With temperature oscillations as high as $8^{\circ} \mathrm{C}$, it appears that the longer the time which the test section stays in the tilted position, the greater the degree to which the liquidus is washed by the melt. Also, there is more time for cooling to occur when the test cell is in proximity to the horizontal. Therefore, with the longer rocking rate, parcels of cold and warm fluid were more efficiently exchanged in the melt, thereby increasing mixing and producing a nearly isothermal melt toward the end of the experiment. Maximum melt temperature differences at two hours were less than $2^{\circ} \mathrm{C}$.

In order to compare the overall enhancement of energy transfer and mixing, the mean melt temperatures of the static experiment and each rocking experiment were 


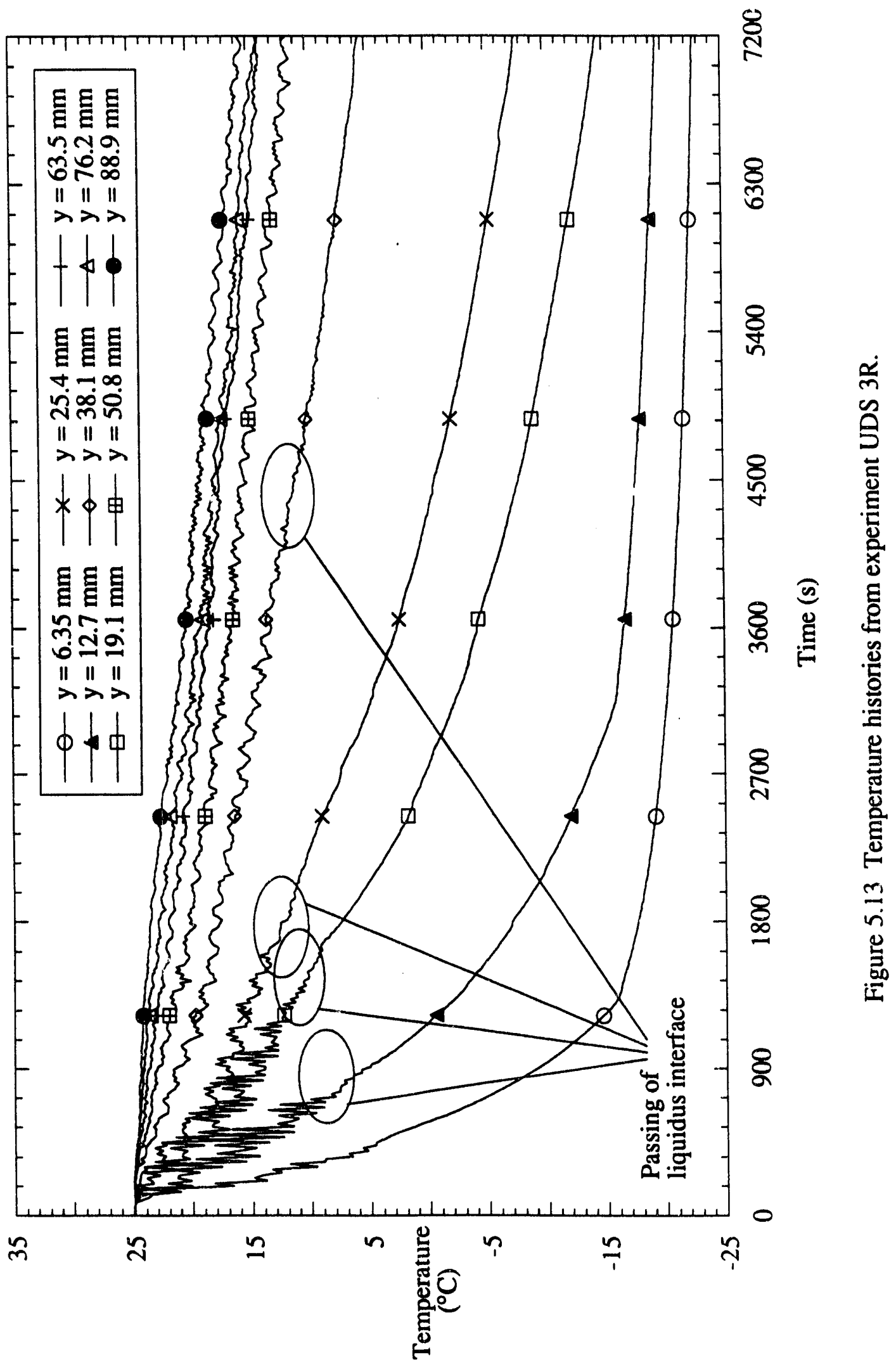




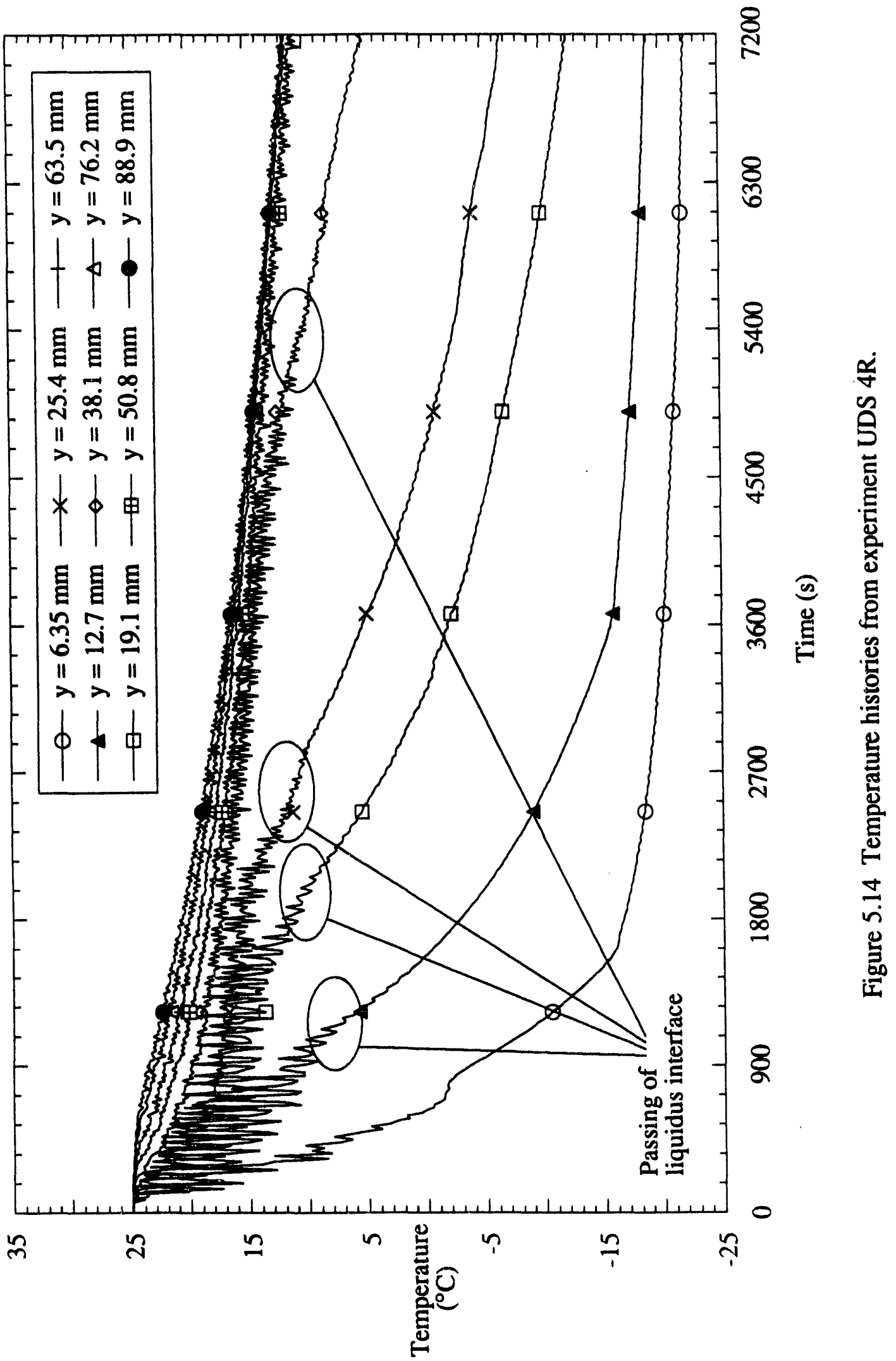


juxtaposed in Figure 5.15. Specifically, the mean melt temperature obtained from the upper four thermocouple locations ( $y=50.8,63.5,76.2$, and $88.9 \mathrm{~mm}$ ) were plotted for each time step. Not surprisingly, the melt temperature of the static case was warmer than for all rocking cases. Whereas heat transfer was accomplished only by conduction and double-diffusive convection in the static case, forced convection arising from the rocking motion acted to enhance heat transfer from the melt. In general, when the rocking rate was two cycles per minute, increased rocking amplitude decreased melt temperature. At two hours, the decrease in melt temperature between the static case and UDS 3R was about $0.5^{\circ} \mathrm{C}$ and the decrease between UDS $3 \mathrm{R}$ and UDS $2 \mathrm{R}$ was about $1.2^{\circ} \mathrm{C}$. However, the decrease between UDS $2 R$ and UDS $1 R$ was a relatively low $0.2^{\circ} \mathrm{C}$. The finding indicates that for a rocking rate of two cycles per minute, increasing the rocking amplitude eventually reaches a point of diminishing returns a:d UDS IR is only a slight improvement over UDS $2 R$. Comparing UDS $1 R$ with UDS $4 R$, the higher rocking rate improved heat transfer only for the first 45 minutes of the experiment. For the latter stages of the experiment, the slower rocking rate of one cycle per minutes allows longer sweeping of the liquidus front, thereby increasing chilling of the melt.

In order to quantify temperature fluctuations for the later stages of each experiment, an effective standard deviation was calculated for selected cooling curves. For each UDS experiment, linear regression was used to fit a line to the melt temperature histories from 3600 to 4350 seconds, which included 50 data samples. Within this time period, cooling curves were very nearly linear, and a standard deviation was based on the difference between the actual temperature and the linear curve fit. Values were then used as a measure of the severity of temperature fluctuations at a particular location.

Figure 5.16 shows the standard deviation, $\sigma$, for the static and four rocking UDS experiments. In the static experiment, fluctuations are largest near the mushy region and decrease with increasing distance into the melt. These fluctuations resulted from rising 


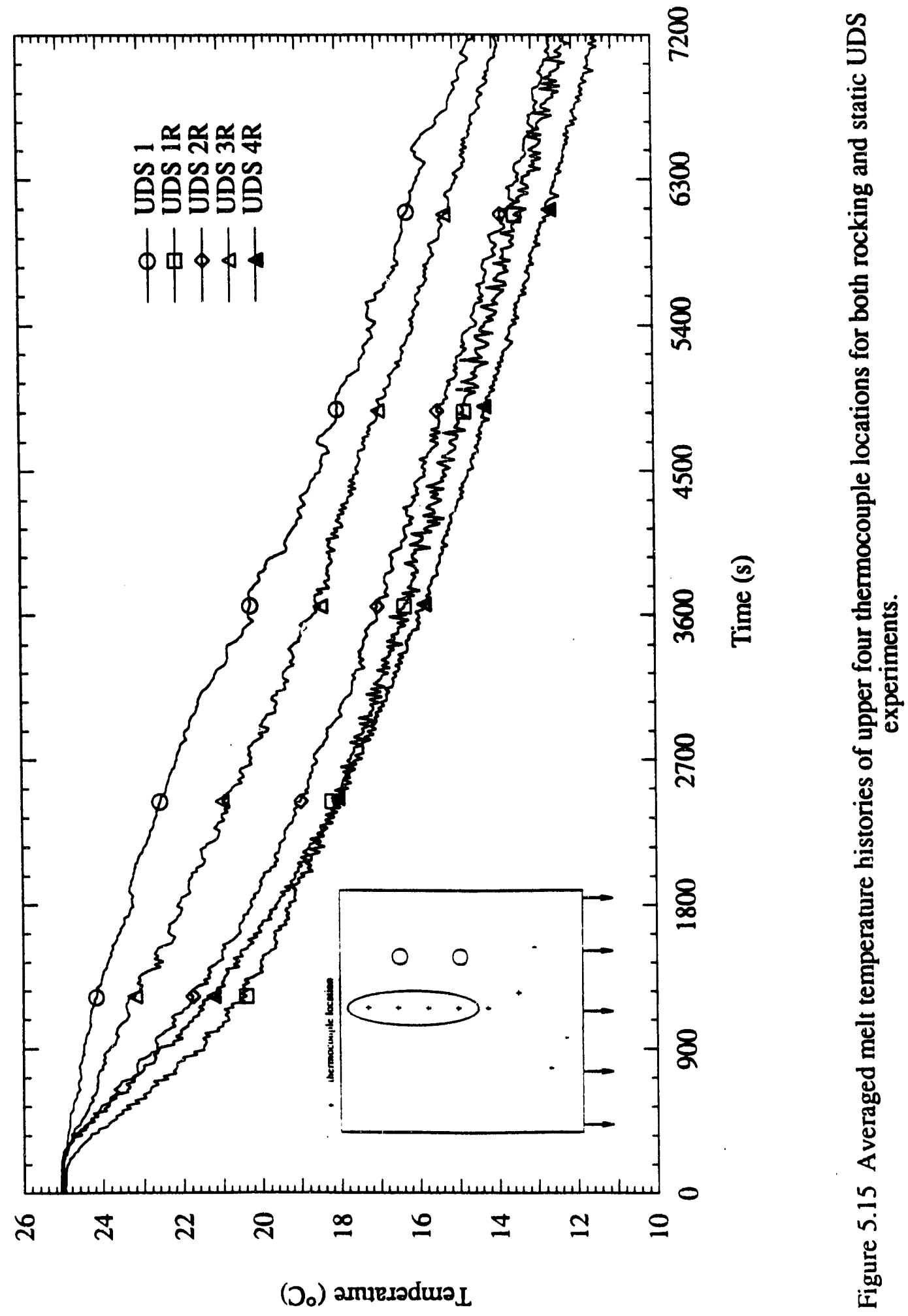




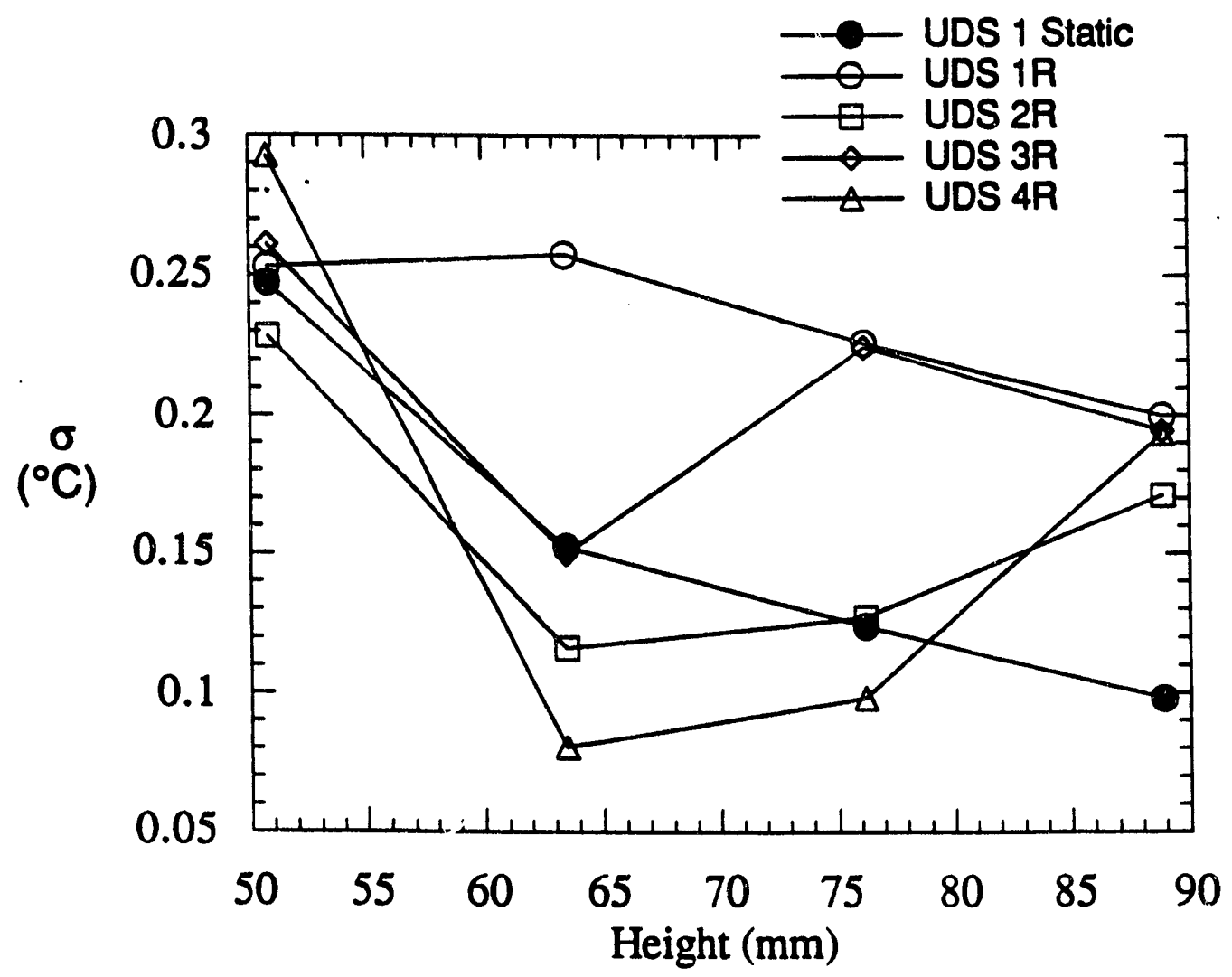

Figure 5.16 Standard deviations of temperature fluctuations in the melt for unidirectional solidification from 3600 to $4350 \mathrm{~s}$. 
and falling buoyant parcels of fluid associated with salt fingering and pluming (Fig. 4.5). On the other hand, experiment UDS $1 R$ was characterized by a large and relatively uniform fluctuation of around $0.25^{\circ} \mathrm{C}$. In the rocking experiments, fluctuations arose, not from salt fingering and pluming, but from bulk fluid motion generated by the agitation. Other than at $y=63.5 \mathrm{~mm}$ where $\sigma$ of UDS $3 R$ is $\sim 0.15$, the standard deviations of runs with amplitucies of $15^{\circ}$ and $45^{\circ}$ are similar; however, the fluctuations of run UDS $2 R$ were substantially less severe than the other rocking cases. In addition, the existence of a nearly quiescent region at the center of the melt is clearly revealed. Finally, the fluctuations associated with UDS $4 R$ exhibited the greatest variation in standard deviation with respect to height. With standard deviations higher or as high as 'ne previous cases in the periphery of the melt, rocking at one cycle per minute produced a very calm, isothermal melt core which had lower fluctuations than any other run. These findings support earlier evidence which suggested that by increasing the time during which the test section was near its maximum tilt, the longer rocking cycle prolonged advection at the liquidus interface, thereby facilitating more effective sweeping of the interface.

As was done under static cunditions, fluid samples were drawn from the melt at certain times during the rocking experiments to determine the mass fraction of $\mathrm{NH}_{4} \mathrm{Cl}$. In comparison to composition measurements taken during UDS 1 (Fig. 4.14), all rocking experiments contained melts which were extremely well mixed. For example, starting with the initial value of $27 \% \mathrm{wt}$. $\mathrm{NH}_{4} \mathrm{Cl}$, the melt compositions of UDS $1 \mathrm{R}$ gradually dropped over time with the values at $y=50.8$ and $76.2 \mathrm{~mm}$ usually within $0.1 \%$ wt. of each other (Fig. 5.17a). This evidence is further testament to the highly efficient mixing provided by the rocking. Also encouraging is the fact that, after two hours of solidification under rocking conditions, the composition of the melt was approximately $25.0 \%$ wt. $\mathrm{NH}_{4} \mathrm{Cl}$, which is a full percentage point above the melt composition of the 


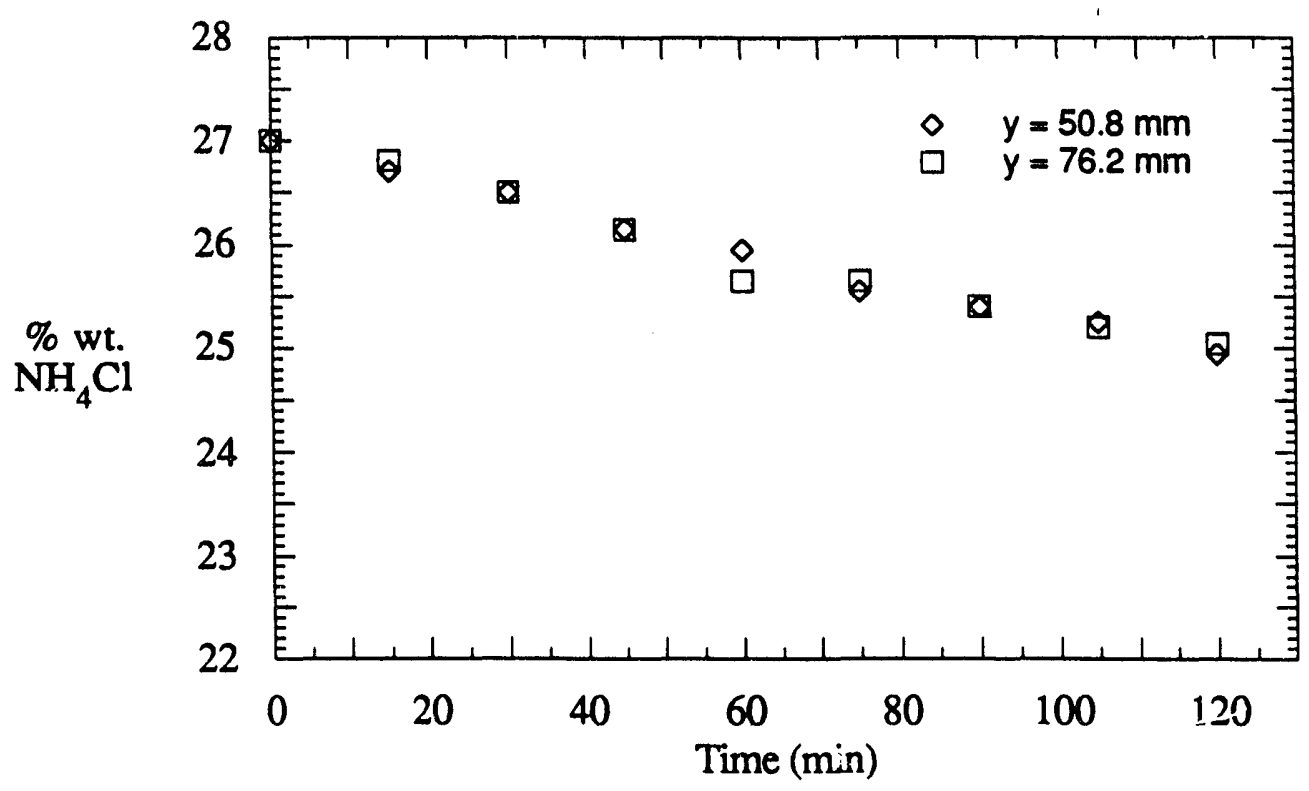

(a) UDS 1R

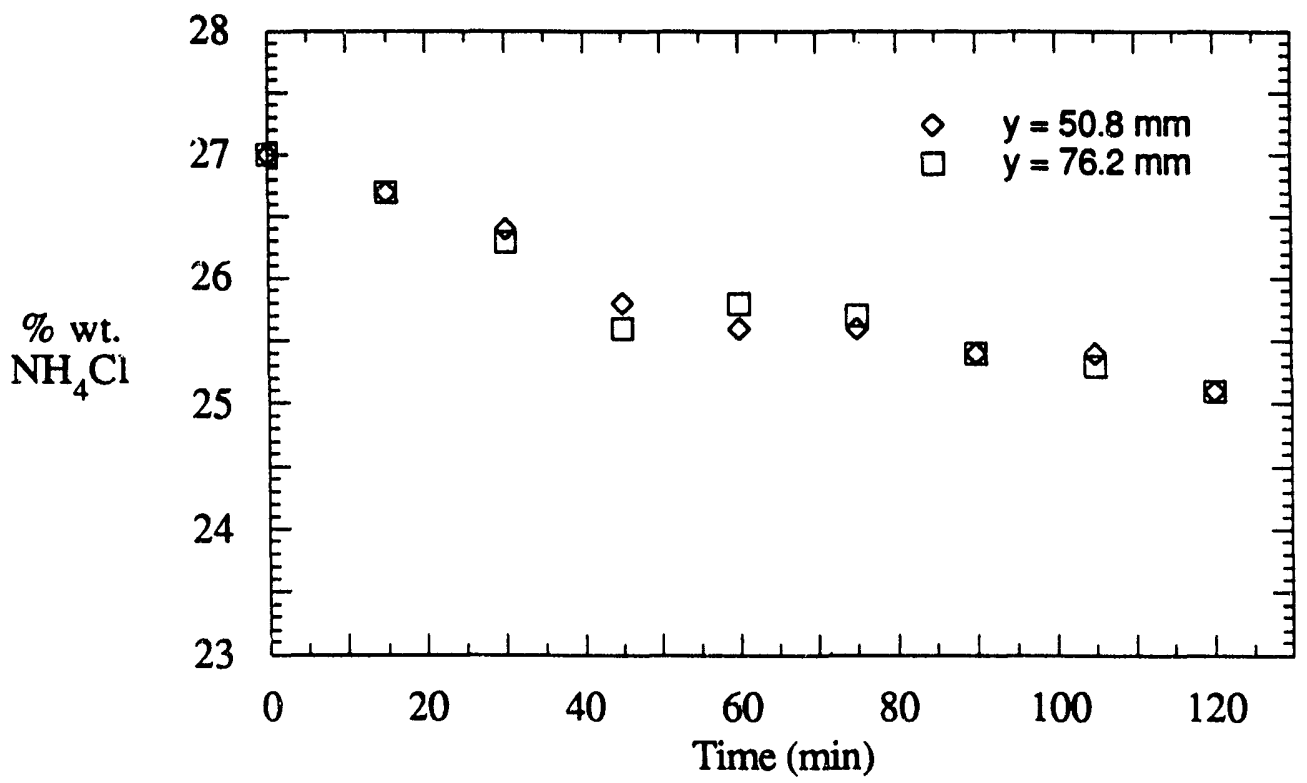

(b) UDS 4R

Figure 5.17 Liquid composition of ammonium chloride for experiments (a) UDS $1 R$ and (b) UDS 4R. 
static cast at the same time. In other words, the rocking motion is actually reducing overall macrosegregation through its suppression of channels.

Other rocking cases show similar results (Figs. 5.17b, 5.18). Excellent mixing was realized using both $15^{\circ}$ and $30^{\circ}$ amplitudes (Fig. 5.18), but as the rocking amplitude decreased from $45^{\circ}$ to $15^{\circ}$, the final melt composition at two hours decreased, approaching the value obtained under static conditions. Finally, the composition data from experiment UDS $4 \mathrm{R}$ (Fig. 5.17b) again showed excellent mixing with even a slightly higher concentration after two hours. The final species composition measurement was $25.1 \%$ wt. $\mathrm{NH}_{4} \mathrm{Cl}$.

A comparison between the liquid composition measurement for each UDS experiment is presented in Figure 5.19. The curves were generated by exponentially fitting a line through the data at $y=50.8 \mathrm{~mm}$. In general, all rocking cases were less severe in segregation of $\mathrm{NH}_{4} \mathrm{Cl}$ than their static counterpart. When the rocking rate was two cycles per minute, higher rocking amplitudes resulted in higher $\mathrm{NH}_{4} \mathrm{Cl}$ concentrations in the melt, indicating less severe macrosegregation from the mushy region. Consistent with mixing trends observed in the cooling curves of the melt, case UDS $4 R$ had yet a slightly higher composition than UDS $1 R$, further illustrating the advantages of using the longer rocking rate with a $45^{\circ}$ rocking amplitude.

\subsubsection{Effect of Rocking on Channeling}

A description of the mushy region for experiment UDS $1 \mathrm{R}$ had been given (see section 5.2.1), but the overall effect on the formation of channels for the remaining rocking experiments has not been presented. When the rocking amplitude was $45^{\circ}$ and the rocking rate was one cycle per minute (UDS $1 R$ ), approxinately four small channels formed. They were not surrounded by a thin crystalline sheath and did not have a volcanic structure at their mouths, such as found with statically grown channels. In 


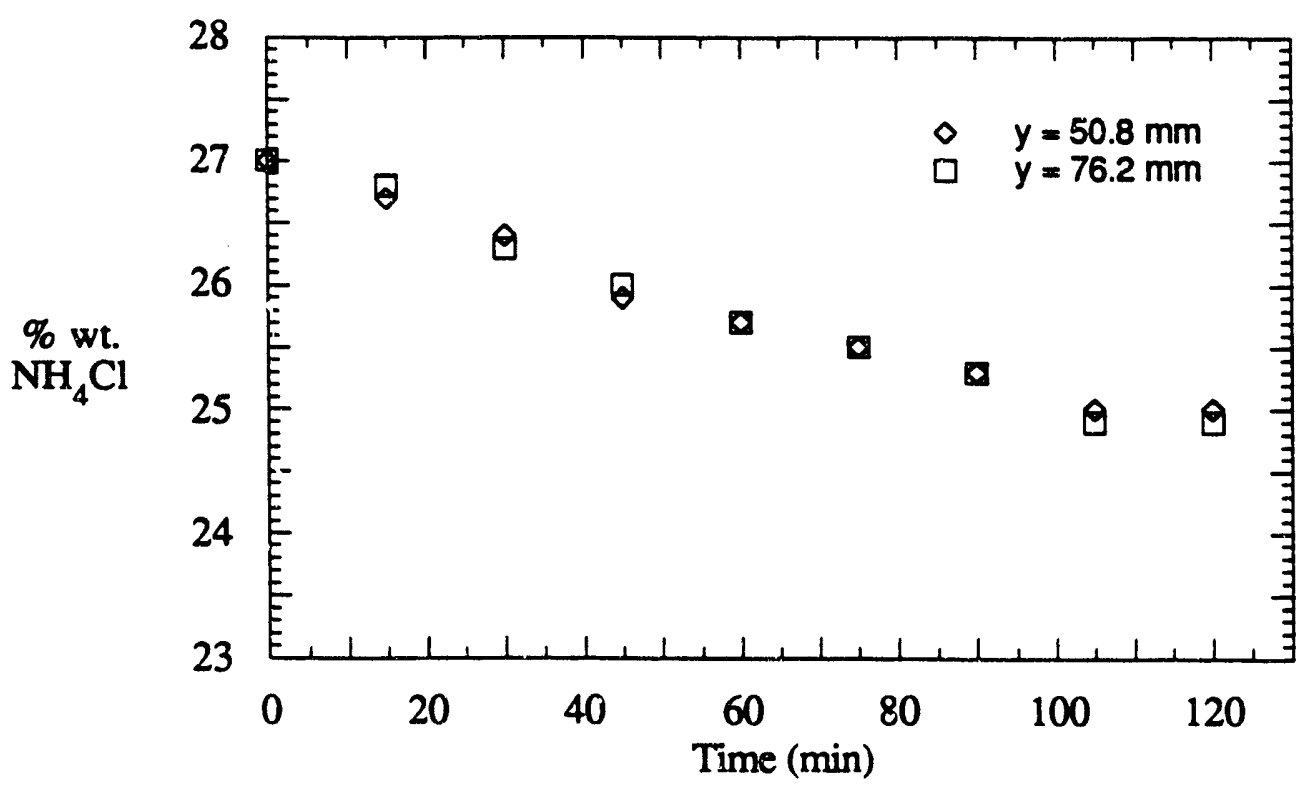

(a) UDS 2R

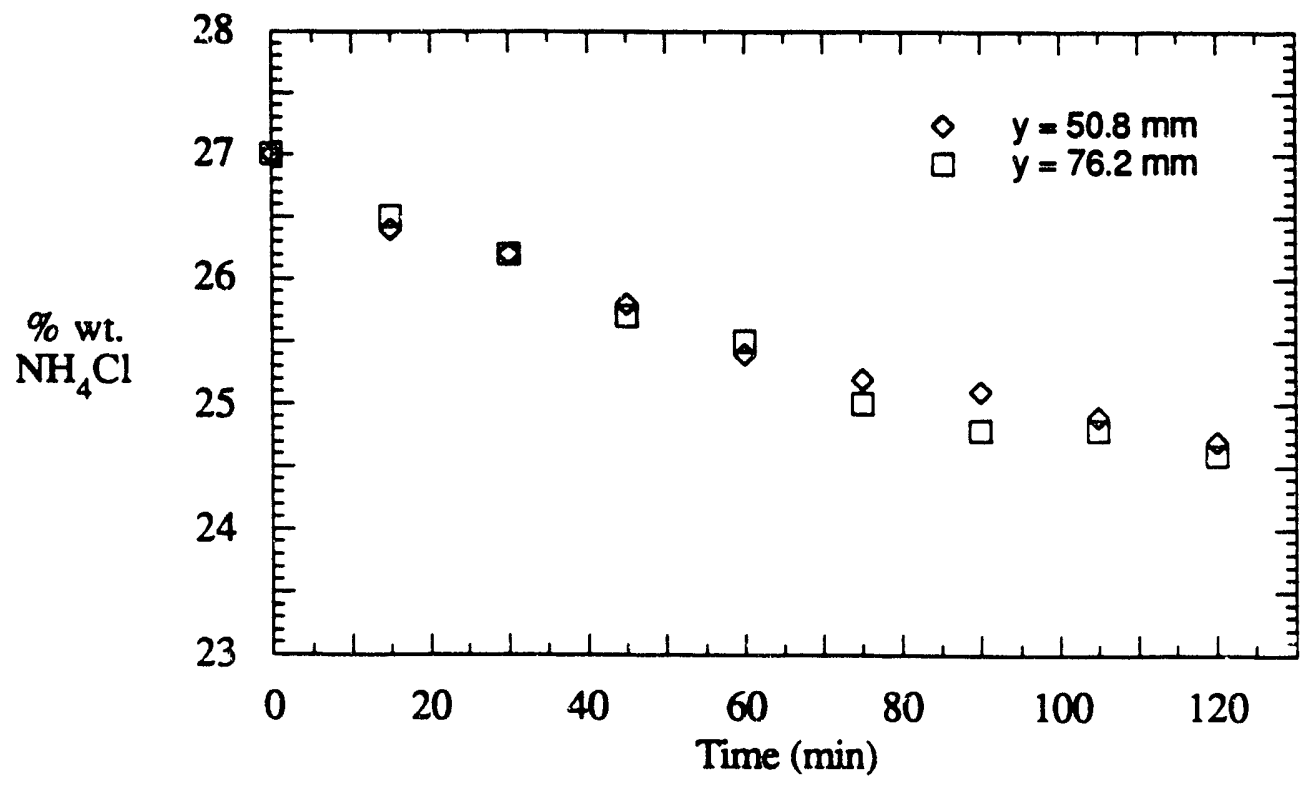

(b) UDS 3R

Figure 5.18 Liquid composition of ammonium chloride for experiments (a) UDS 2R and (b) UDS 3R. 

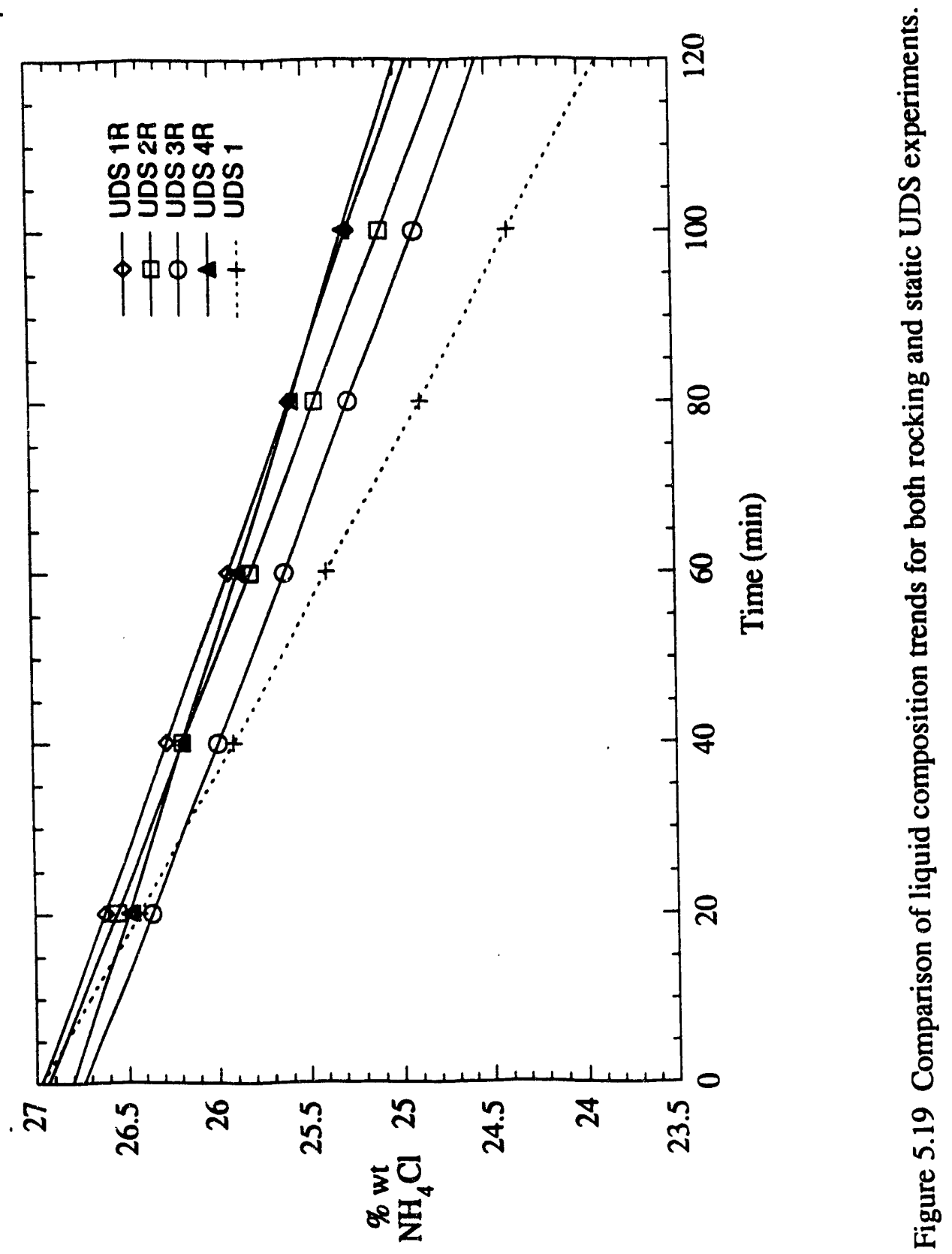
addition, the mushy region consisted of individual columnar dendrites which were not associated with any ordered grain structure. When the rocking amplitude was changed to $30^{\circ}$ (UDS 2R), four small channels again formed, and overall, there was little difference in mushy region and channel morphology between the two cases. When the rocking amplitude was changed to $15^{\circ}$ (UDS 3R), small, but noticeable, changes appeared in the mushy region morphology. Three larger holes which developed in the mush formed crystalline sheaths but did not form voicanic structures around their mouths, and these channels began to approach the size and structure of statically grown channels. The mushy region, consisting primarily of uniquely oriented dendrites, did have small grain regions near the outer walls. Overall, the mushy region of case UDS $3 R$ had begun to resemble mushy regions found in static casts. Finally, when the mold was rocked at one cycle per minute and with an amplitude of $45^{\circ}$, extremely promising results ensued. Though columnar, the crystalline structure again consisted of randomly oriented primary dendrites; however, no larger channels developed in the mush. Only three small holes, no larger than the primary dendritic arm spacing, formed within the crystalline structures. Therefore, there seems to be an optimum rocking rate at which channels can be eliminated.

Sample and Hellawell (1982) found that a precessional movement at $30^{\circ}$ and 5 rpm eliminated channels in a UDS arrangement. Although the present rocking mechanism cannot rotate at those higher rates, it is interesting to note that a slower rocking motion of one cycle per minute and $45^{\circ}$ performs better than a rocking rate of two cycles per minute. Further investigations with rocking rates of 0.5 cycles per minute and slower could reveal an optimal condition. However, for the limited data available, it is difficult to speculate what that optimum may be. 


\subsection{Side and Bottom Wall Chilling}

\subsubsection{Qualitative Observations}

As was done when investigating the effects of rocking on UDS casts, a range of rocking amplitudes was used to investigate macrosegregation patterns associated with solidification in test section II under rocking conditions. As shown in Table 5.1, three rocking amplitudes of $45^{\circ}, 30^{\circ}$, and $15^{\circ}$ with a constant rocking rate of two cycles per minute were studied. The experiment with the most severe rocking conditions, MDS IR, was chosen for the flow visualization study. Again, the test section was rocked at least a full minute before application of the coolant to the heat exchangers to assure that inertially driven flow conditions existed in the melt at the start of solidification.

Very similar to the static experiments, the incipience of solidification with rocking was characterized by strong thermal buoyancy, rapid crystal growth on the heat exchangers, and robust salt fingering. The rapid drop in temperature of the heat exchangers produced strong falling thermal boundary layers on the side walls, as well as a thermal sweeping motion along the bottom. Within 15 seconds after applying coolant, crystals began to form on the heat exchanger surfaces rejecting water-rich fluid which tended to rise, thus producine sali fingers along the bottom surface. However, those first fingers were rapidly carries ielt and mixed by convection patterns produced by the rocking motion (Fig. $5.20 \mathrm{c}, \ldots . . .$. strong convective motions in the melt also acted to dislodge and break many of the nascent dendrites that grew on the side, resulting in a large and dense slurry which coalesced in the lower regions of the cavity. Taken as the test section swept from the left up to the vertical position, Figure 5.20a shows a concentration of this slurry in the lower left corner of the melt. Originally collecting in the lower left corner when the test section was tilted to the left, the slurry was driven by inertial convection across the bottom growth front toward the right as the test section rocked to the vertical. Eventually, as the test section rocked to the right, the slurry of 


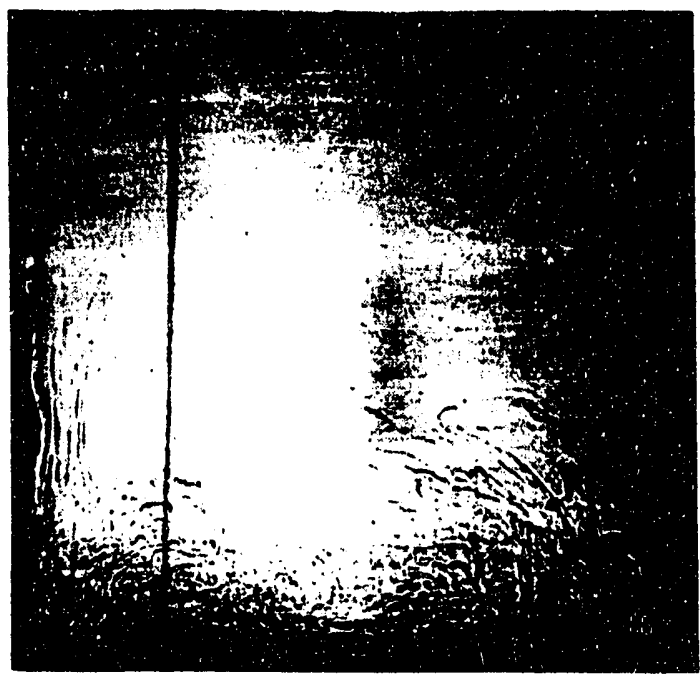

(a)

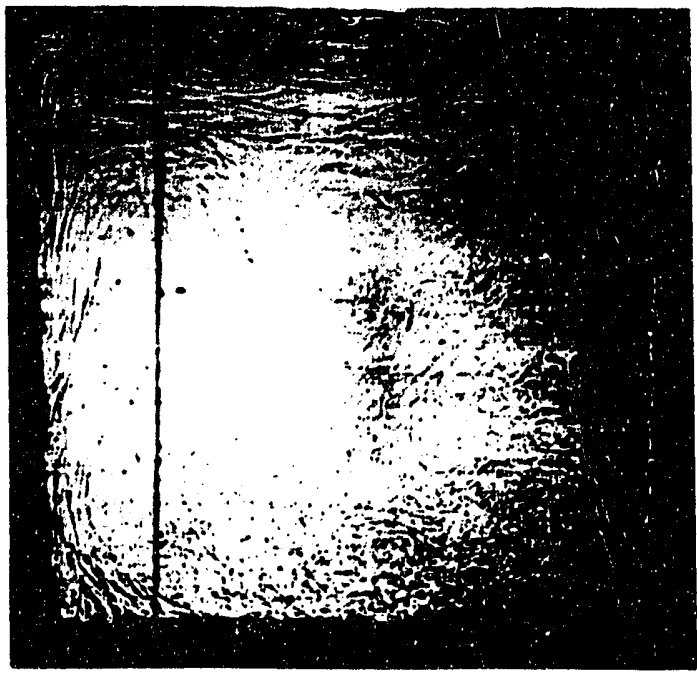

(c)

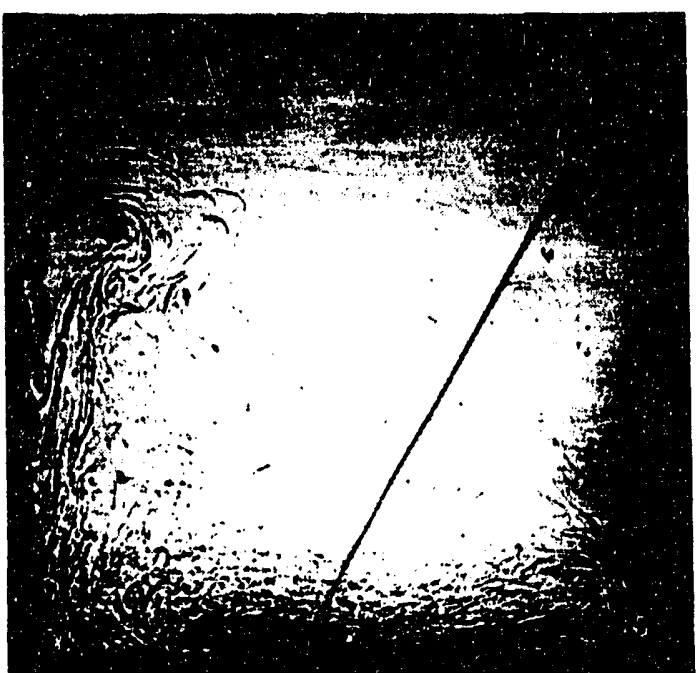

(b)

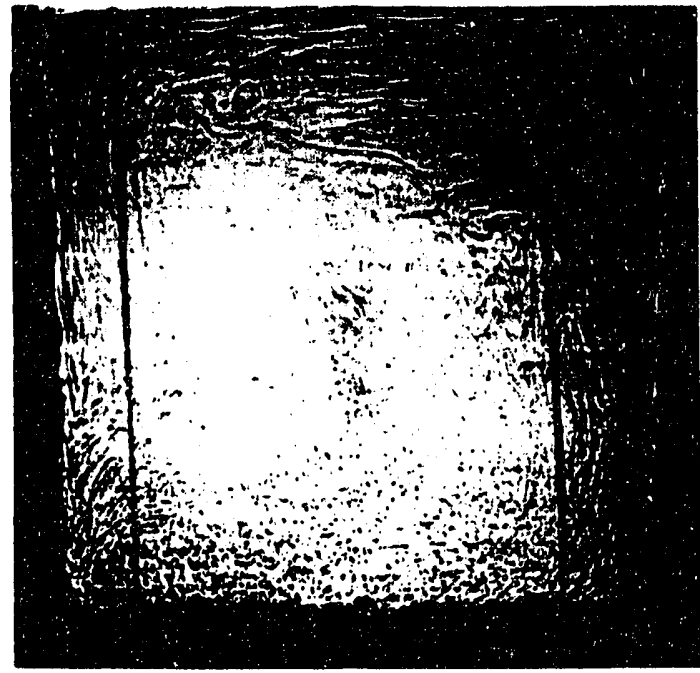

(d)

Figure 5.20 Shadowgraph images of early solidification for case MDS $1 R$ : (a) $1\left(\theta=2^{\circ}\right)$, $1.5\left(\theta=30^{\circ}\right),(c) 3,\left(\theta=1^{\circ}\right)$ and (d) $4 \min \left(\theta=-4^{\circ}\right)$. 
dendrite fragments collected in the lower right corner of the cavity. At 1.5 minutes, many crystals from the slurry had settled onto the bottom wall mushy region, decreasing the slurry density (Fig. 5.20b). In addition, strengthening convective motions in the melt began to disperse both salt fingers and the slurry throughout the cavity. By three minutes into the experiment, a well established rotational flow was carrying both thermal energy and species around the periphery of the melt (Fig. 5.20c). Figure 5.20c clearly shows the inertially induced clockwise flow of fluid around the cavity as the test section rocks from the right to the vertical position. Also shown is an active slurry concentrated in the lower right corner of the test section; but by this time, the free-floating crystals were not confined to the lower region, as many were advected throughout the melt by vigorous buoyancy and inertially driven convection. By four minutes, the slurry in the lower regions of the cavity had a lower concentration of dendrite fragments. However, the fragments continued to be advected throughout the melt (Fig. 5.20d). Strong convection continued, and Figure 5.20d shows the counterclockwise flow in the cavity when the box rocked from the left up to the vertical.

Vigorous salt fingers or solutally driven plumes originated from crystal growth on all walls, but were most prevalent along the bottom wall early in the experiment. Plumes appeared from the side wall mush at approximately two minutes into the experiment (Fig. $5.20 \mathrm{c})$ and were quickly mixed into the melt. The strong fluid motions produced by the rocking acted to stretch and drag the fingers/plumes throughout the cavity, thereby facilitating the well mixed conditions of Figure 5.20d. Also, throughout the early stages of the experiment, the larger crystals of the slurry collected on the bottom mushy zone, promoting a distinct equiaxed region which had an appearance very much like freshly fallen snow.

Past five minutes, continued strong convective motions efficiently mixed the melt and suppressed the development of double-diffusive convection cells at the top of the 
cavity (Fig. 5.21). The concentrated slurry which had developed in the lower regions of the cavity in the early stages of the experiment had dispersed throughout the melt by five minutes (Fig. 5.21a). In addition, the convective fluid rotation continued to drive fluid, energy, and species to all corners of the melt. During the static chill experiment, waterrich interdendritic fluid generated in the side wall mushy region began to seep into the upper comers of the melt at about 6.5 minutes into the experiment (Fig. 4.15). However, with rocking, interdendritic fluid within the side wall mush does not seep from the top of the mushy region; rather, as the test section tilts to one side, the water-rich fluid rises from the mushy region at several locations along the side wall liquidus interface. Figure $5.21 \mathrm{~b}$ shows these strong outflows in the upper regions of the left side wall. These outflows, being very water-rich, are strongly buoyant, overcoming both thermal and inertial fluid motions to rise to the top of the cavity. The water-rich fluid collects in the upper melt, and with a continuous supply of fresh water-rich fluid transferred from the side wall mushy rose to the upper melt, a layer of water-rich fluid formed at the top of the cavity. This layer, clearly seen at nine minutes (Fig. 5.21c), oscillated back and forth across the top of the test section with the rocking motion, impinging on the side walls and mixing with the underlying melt. Figure $5.21 \mathrm{c}$, taken as the test section rocked to the left, shows the water-rich layer beginning to ascend to the right, where it eventually impinges on the right side mushy region. Similarly, Figure 5.21d, taken at approximately 11 minutes, shows conditions of the water-rich layer immediately after it impinged on the side wall. Note the vigorous folding and roiling of the buoyant layer as it mixed with the underlying melt. Although some solutal stratification developed in the melt, it was this mixing process arising from the rocking motion that effectively suppressed the formation of distinct double-diffusive convection cells and concomitant macrosegregation. It should be mentioned that, as with the rocking UDS case, flow characteristics in the rocking side wall chill experiments were highly three-dimensional. In fact, Figure 5.21d 


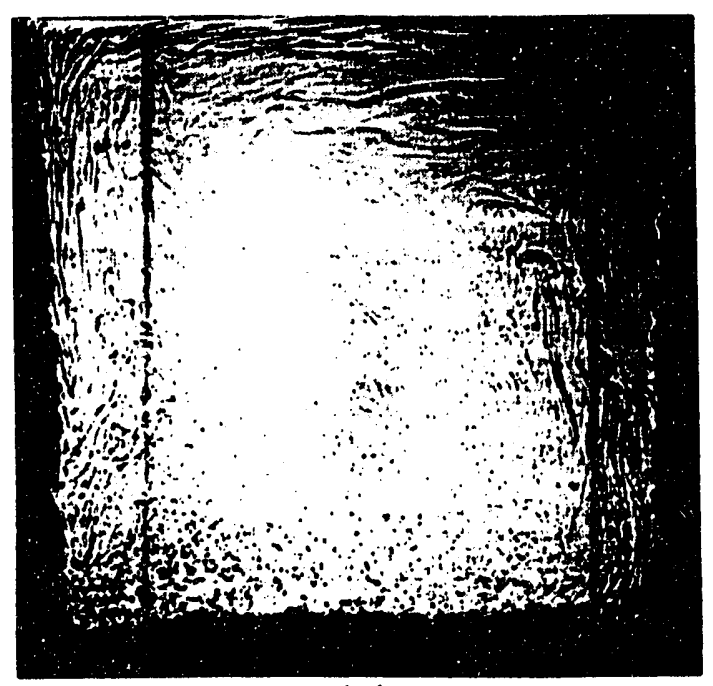

(a)

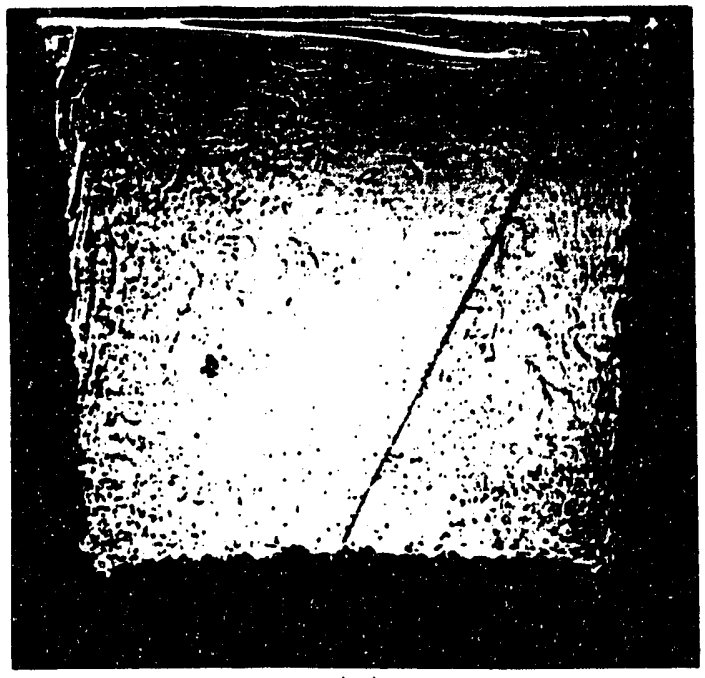

(c)

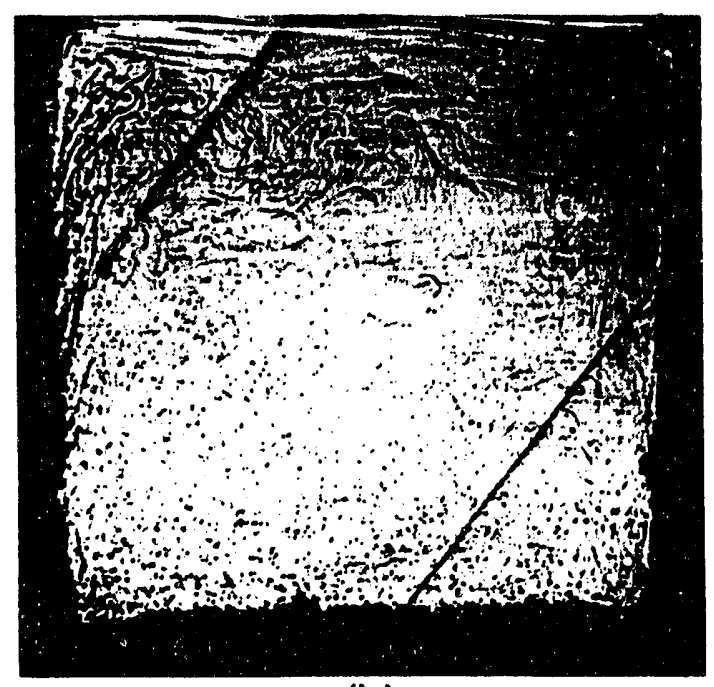

(b)

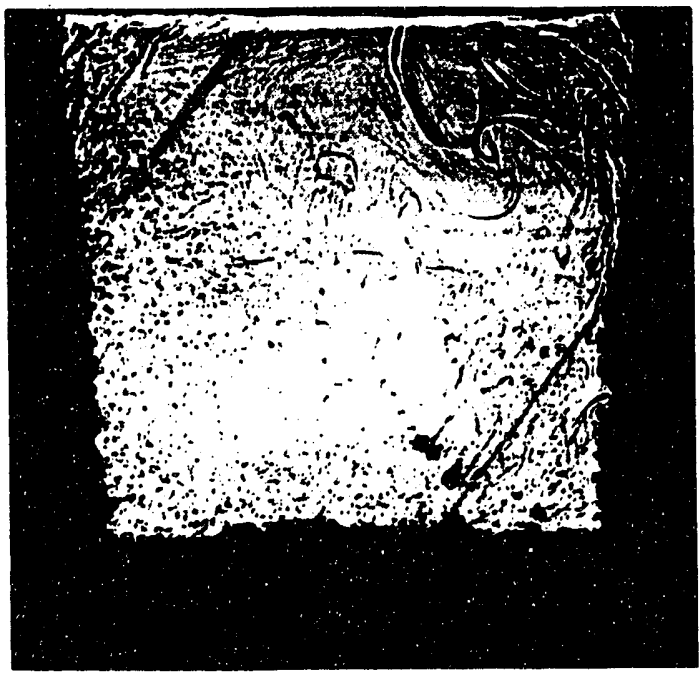

(d)

Figure 5.21 Shadowgraph images for case MDS 1R: (a) $5\left(\theta=0^{\circ}\right)$, (b) $6.5\left(\theta=37^{\circ}\right)$, (c) $9\left(\theta=27^{\circ}\right)$, and (d) $11 \mathrm{~min}\left(\theta=38^{\circ}\right)$. 
suggests some of this 3-D behavior in the upper right corner of the cavity, as the waterrich layer mixes with the melt, forming rolling, folded vortices.

The dendrite fragments constituting the widely distributed slurry region from 5 to 11 minutes originated primarily from the side wall mush. As in the static case, some crystals were rejected from deep within the mushy region by solutal upwelling; however, most crystals in the slurry originated as crystalline conglomerates which broke free from the side wall mushy region. Figure 5.21d shows three such large structures descending in the lower right corner. The conglomerates often descended to the mushy region floor, breaking into smaller crystals which were subsequently swept away by convective washing of the interface. However, crystals were not ejected into the melt by robust salt fingers or plumes rising from the bottom mushy region, as was the case in the static experiment, MDS 1.

Surprisingly, the bottom growth front was free of salt fingers and plumes. One possible explanation of their absence is that the highly equiaxed dendritic structure of the mushy region prevented salt fingering. However, this explanation is contradicted by results from experiment MDS 1, which show that strong salt fingering and plume development can arise from equiaxed structures. A more likely reason is that there are too many crystals at the liquidus interface to promote further solicisication. Although there appears to be rapid crystal growth and vigorous salt fingering along the bottom wall early in the experiment, when there is a strong thermal gradient and apparent undercooling, past seven minutes, more crystals fall onto the bottom wall growth front than can be supported by existing thermal gradients. In essence, a thermodynamic superheat exists at the liquidus interface, providing a higher mass fraction of solid than would otherwise be supported by thermodynamic equilibrium. Therefore, no solidification occurred at the interface, and in turn, no water-rich fluid, which would 
promote salt fingering, was rejected. In fact, crystals which fell onto the growth front could well have undergone slight remelting.

From 15 to 45 minutes, crystal morphology was similar to that of the static casts. At 15 minutes, the side wall mushy region was nearly planar, but tilted such that the bottom was slightly thicker than the top. Also, due to settling of dendrites from the slurry, the bottom wall mushy region was planar and horizontal. Flow conditions within the melt continued to be driven by the rocking motion, but the water-rich layer of fluid at the top of the cavity grew larger and continued to oscillate back and forth across the test section. Figure 5.22a, taken at 15 minutes, shows the water-rich fluid layer shortly after it had impinged on the upper right mush, resulting in vigorous mixing with melt. The slurry persisted throughout the melt at 15 minutes, and as dendrites settled onto the mushy region, new crystal conglomerates were dislodged from the side wall, thus contributing to the slurry. Several such conglomerates can be seen near the plumb line in the lower right corner of Figure 5.22a. At 20 minutes into the experiment, the water-rich layer at the top of the cavity had grown larger, and it seemed to be developing distinct horizontal interfaces (Fig. 5.22b). However, such trends were continually opposed by convective mixing in the melt. Figure $5.22 \mathrm{~b}$ also shows the vigorous rejection of waterrich fluid from the right side wall when the test section was tilted to the right. These salt fingers escaped from many A-segregate type channels in the side wall mush which, in turn, efficiently released the interdendritic potential contained within the mushy regions. These channels differed little from channels grown under static conditions.

After 31 minutes, the solidification rate decreased, as solutal discharges and solutal gradients in the melt weakened (Fig. 5.22c). The water-rich fluid at the top of the cavity occupied approximately the upper quarter of the melt region; however, as Figure $5.22 \mathrm{c}$ shows, the visual distinction between the upper layer and the melt, which had been pronounced at earlier times, was more obscure at 31 minutes. This development resulted 


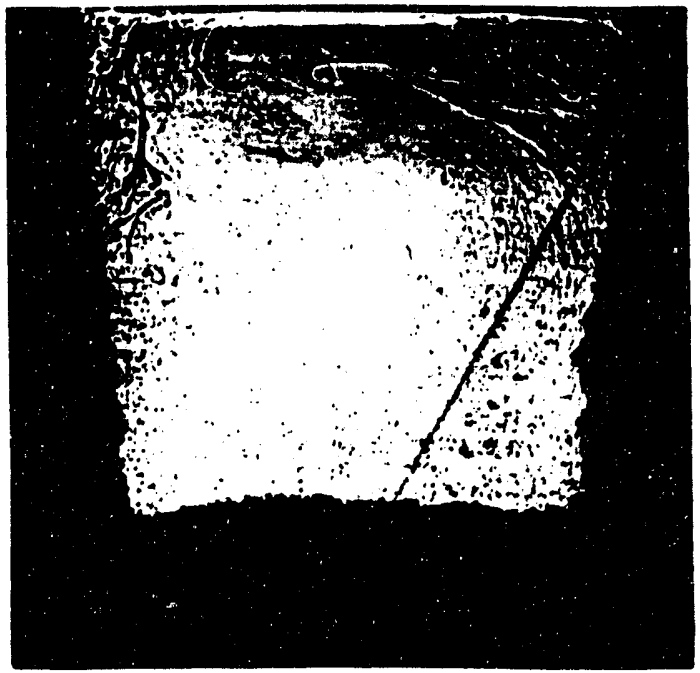

(a)

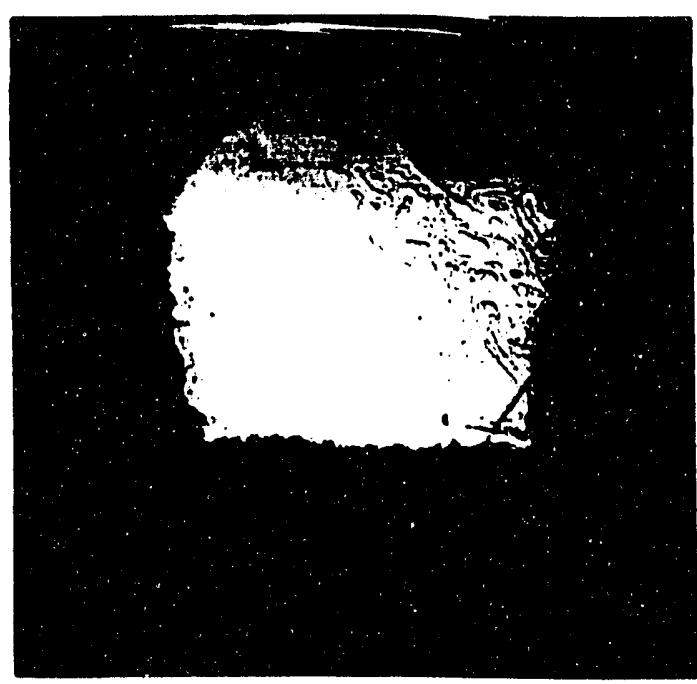

(c)

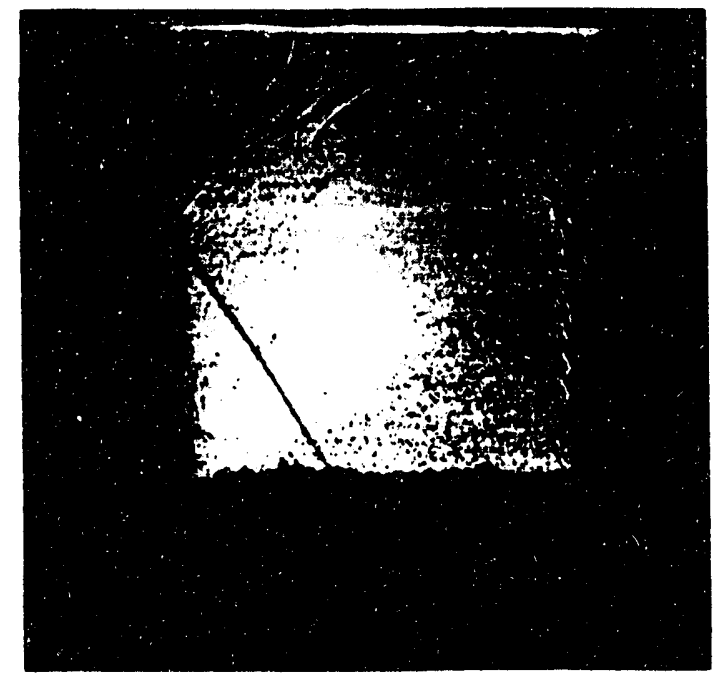

(b)

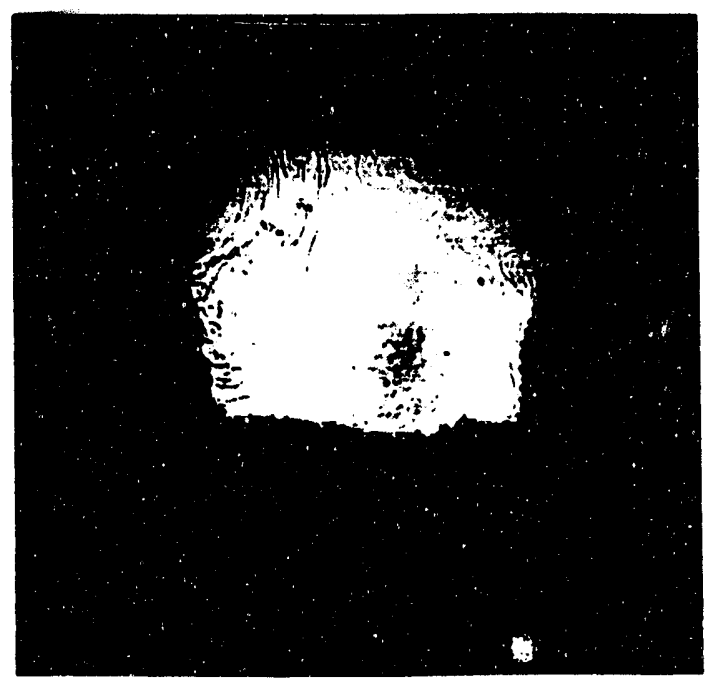

(d)

Figure 5.22 Shadowgraph images for case MDS 1R: (a) $15\left(\theta=30^{\circ}\right)$, (b) $20\left(\theta=-31^{\circ}\right)$, (c) $31\left(\theta=37^{\circ}\right)$, (d) $44 \min \left(\theta=4^{\circ}\right)$. 
from both the effective agitation present in the first 30 minutes of the experiment and the decreased solidification rate which rcduced the supply of water-rich fluid to the upper layer. Owing to the concentration of water-rich fluid in the upper sections of the melt, solidification at the upper side wall mushy region was retarded. Though no remelting is believed to have occurred, the growth front in the upper mush progressed slowly relative to growth at the lower side wall, thereby resulting in a steep side wall liquidus interface. However, in contrast to the static casts and due to efficient mixing in the melt, the mushy region remained on the entire side wall and did not melt away in the upper sections of the cast. At 44 minutes, the water-rich layer in the upper melt persisted and water-rich fluid continued to issue from channels in the side wall mush (Fig. 5.22d). Also, the solidification rate continued to be larger at the lower side wall liquidus than at the upper liquidus. The slurry was virtually nonexistent at 44 minutes. However, the bottom wall mush consisted of a large equiaxed structure formed by the continuous settling of dendrites from the slurry during the first 40 minutes of the experiment. Toward the later stages of solidification, condensation on the outside of the view plates indicated that the upper region of the melt was cold and that a thermal inversion had developed.

At one hour, relatively strong discharges continued to issue from channels in the side wall mush, and although no double-diffusive interfaces were present, the melt seemed to be stratified (Fig. 5.23). Although no crystal remelting had occurred, waterrich fluid in the upper sections of the cavity suppressed solidification at the upper side wall growth, front such that the side wall mushy region was considerably thicker toward the bottom of the cast. The slurry region was nonexistent, and along the bottom growth front, columnar dendrites had begun to grow on the equiaxed base.

Figure 5.24 is a schematic rendition of observations, which juxtaposes castings for the static and rocking cases. Grain morphology and macrosegregation between the two cases are similar. Other than a larger equiaxed zone and a mushy region that covers the 

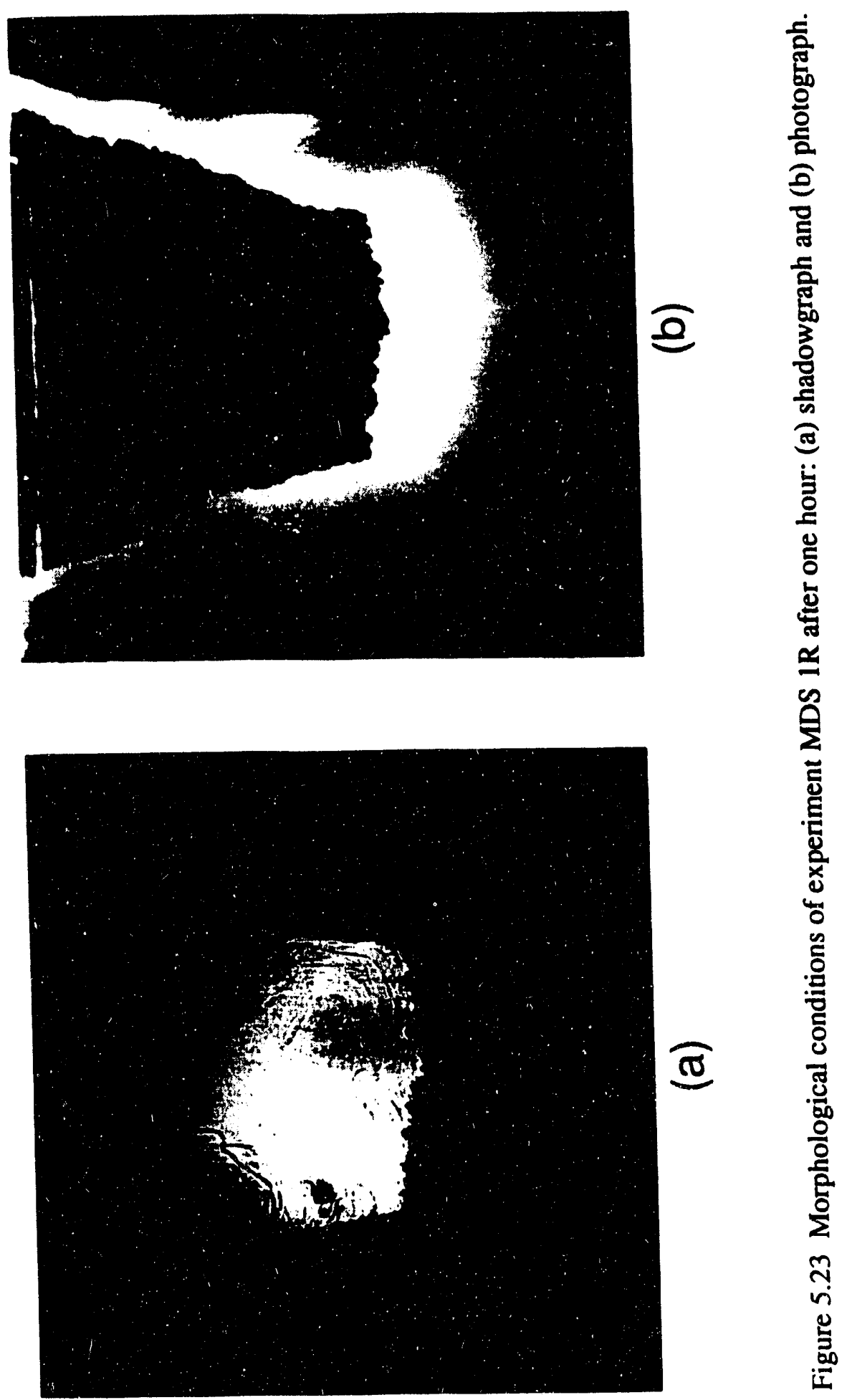


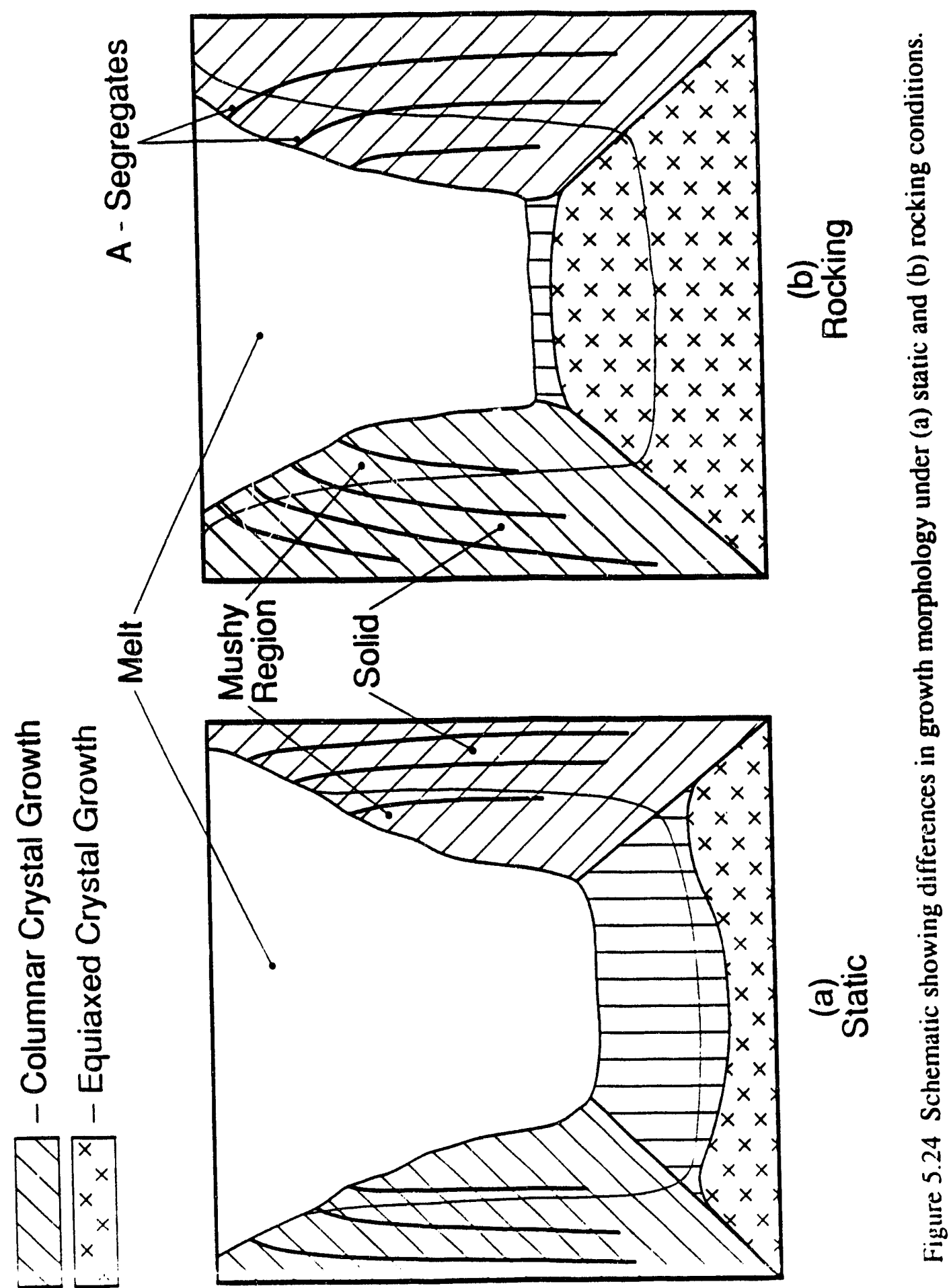


entire growth front, crystal structure associated with rocking is unchanged from static conditions. With respect to the macrosegregation, A-segregate type channels were not changed in my way with rocking, nor was banded segregation eliminated, though it was less severe. However, freckle-type channels growing from the bottom wall were completely eliminated and replaced by an isotropic equiaxed structure. All rocking experiments were stopped shortly after one hour.

\subsubsection{Quantitative Results}

T: mperature histories for case MDS 1R, where the rocking rate was two cycles per minute and the rocking amplitude was $45^{\circ}$, are presented in Figure 5.25. The first five minutes of solidification were characterized by establishment of a shermally stratinied condition in the melt, whereby the coolest fluid collected at the bottom of the crvity. Although the melt temperatures fluctuated sligntl? conditions were generally unaffected by the rocking motion in the early stages of the experiment. However, toward the end of the experiment, excellent mixing did develop in wisich the uppt: four therinocouples recorded temperatures within $2^{\circ} \mathrm{C}$ of each other.

At $y=12.7 \mathrm{~mm}$, the temperature dropped very rapidly to $11.7^{\circ} \mathrm{C}$ at $t=3 \mathrm{~min}$, which is similar to behavior for the static experiments. However, beyond three minutes, the temperature rose steeply for approximately 70 seconds to a vaiue of $15.0^{\circ} \mathrm{C}$. The temperature then decreased and continued to do so for the remainder of the experiment. The question becomes: what mechanism produced this steep thermal rise at $y=12.7$ $\mathrm{mm}$ ?

One possible explanation is that warm fluid from the melt was advected to this location by rocking forces, thereby producing a large temperature fluctuation like that seen in case UDS IR. However, as the test section tilted, and thermal buoyancy at the bottom liquidus interface caused chilled fluid to descend along the interface, the 


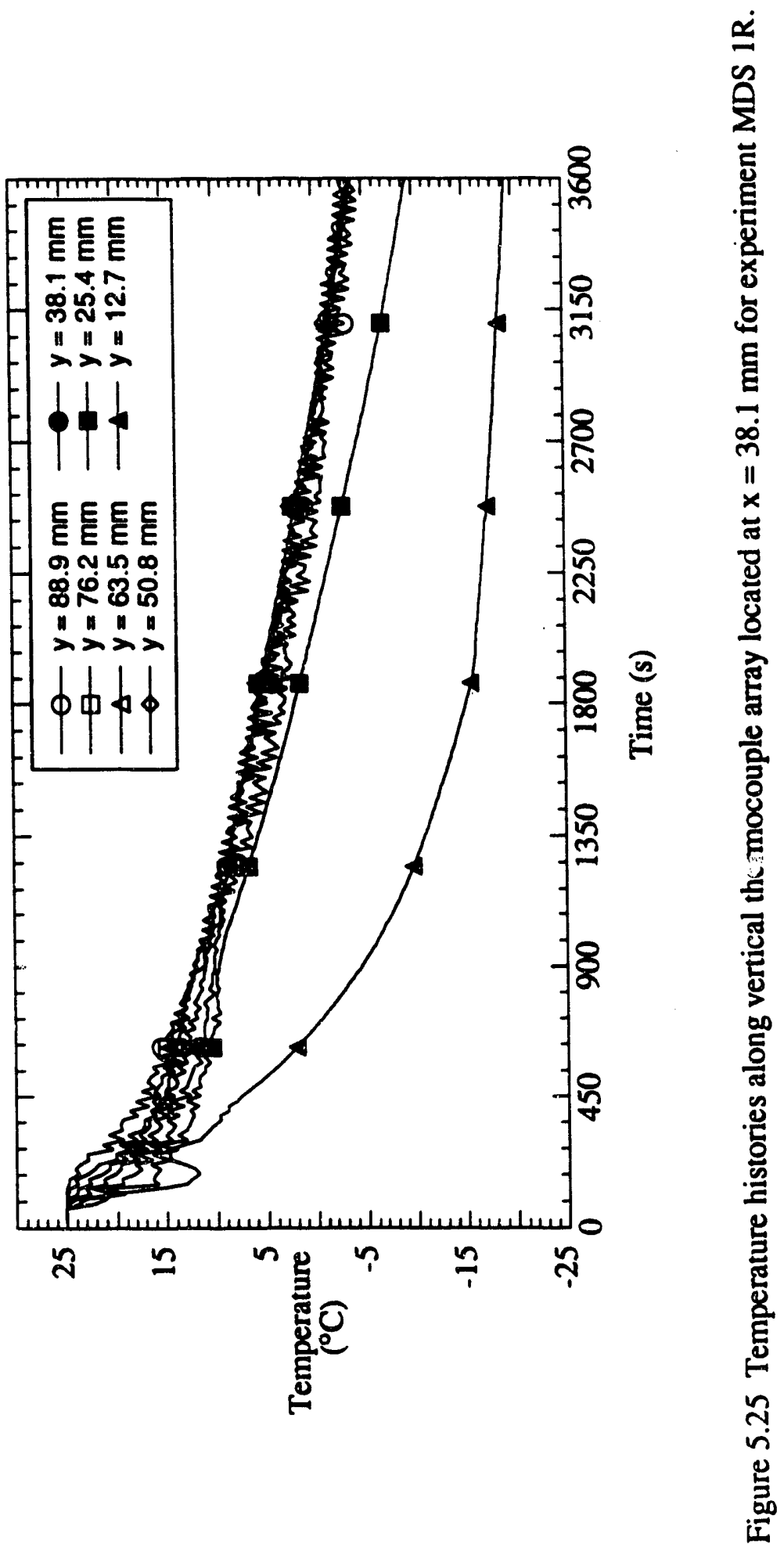


replacement fluid should not come from the core of the melt but from the thermal boundary layers developing on the elevated side wall. Therefore, the replacement fluid was itself chilled and could not raise the measured temperature. Another possible explanations is that the temperature rise is a manifestation of recalescence, a thermodynamic nonequilibrium phenomenon in which a solidifying particle is exposed to an undercooled liquid, thereby producing rapid solidification, a subsequent release of latent heat, and therefore, a noticeable rise in temperature.

Comparing the temperature history at $y=12.7 \mathrm{~mm}$ with the photographic record of the experiment (Fig. 5.20), the position of the thermocouple with respect to the growth front can be tracked. The low temperature of $11.7^{\circ} \mathrm{C}$ at three minutes corresponds to a thermocouple location which is just above the liquidus interface, and the high temperature corresponds to the location of the thermocouple precisely at the liquidus interface. At three minutes into the experiment, it can be assumed that the melt is still relatively close to its original $27 \% \mathrm{NH}_{4} \mathrm{Cl}$ composition, in which case the corresponding liquidus temperature is $18.6^{\circ} \mathrm{C}$. Therefore, it is highly probable that at $11.7^{\circ} \mathrm{C}$, the fluid just above the liquidus interface is undercooled and recalescence may occur. However, with undercooling, several questions arise: 1) with an ample supply of nucleation sites (the slurry), why does homogeneous or big bang nucleation fail to occur just above the growth front; and 2) why was recalescence not observed in the static experiments?

When surrounded by an undercooled liquid, a free floating crystal from the slurry which is located just above the liquidus interface should experience solidification. However, as the crystal solidifies ammonium chloride, it rejects a water-rich fluid which shields it from the salt-rich fluid in the melt. In order for crystal growth to be sustained, $\mathrm{NH}_{4} \mathrm{Cl}$ must penetrate the water-rich buffer which surrounds the crystal, either by advection or diffusion. Because the crystal moves with the flow, advection is minimal, at best. Moreover, the high Lewis number of $\mathrm{NH}_{4} \mathrm{Cl}-\mathrm{H}_{2} \mathrm{O}$ dictates a very slow diffusion of 
$\mathrm{NH}_{4} \mathrm{Cl}$ to the crystal. Therefore, the free-floating crystal tends to grow very slowly and hence to release little latent heat. If, however, the crystal were to become caught in the mushy region at the liquidus interface, drastically different growth characteristics would result.

Once caught in the mushy region, the crystal would be affixed and its development would be influenced by advection along the liquidus interface. The waterrich fluid which originally shielded the crystal from further growth would be advected away and replaced by strongly undercooled, salt-rich fluid. This fresh supply of undercooled fluid at the crystal's solid-liquid interface would promote rapid solidification, thereby releasing latent heat and raising the temperature locally at the liquidus interface. Therefore, it is the strong advective fluid motion present in the rocking case, and absent in static solidification, which triggers the local release of latent heat and, hence, recalescence. As evidenced by crystals which nucleated on thermocouples within the melt, undercooling was present throughout the experiment; although, it became less severe as thermal gradients in the cast decreased.

As was seen with the UDS rocking experiments, sampling related oscillations developed in the cooling curves of the melt owing to the sampling rate of 15 seconds. However, even with the alias, there seems to be a period at around 15 minutes when large temperature fluctuations were absent. This period corresponds to a transition from thermally dominant buoyancy to solutally dominant buoyancy in the melt. Early in the experiment, the density gradients were caused primarily by a pool of cold fluid which formed at the bottom of the cavity and was advected into the melt, thereby producing temperature oscillations. However, as solutal buoyancy began to dominate conditions in the melt at around $t \approx 15 \mathrm{~min}$, a temperature inversion began to occur. During the transition from a positive to a negative vertical temperature gradient in the melt, overall thermal gradients decreased and the temperature fluctuations were damped. Beyond 20 
minutes, the solutal layer of cold, water-rich fluid collected at the top of the cavity which, under the effect rocking, regenerated temperature oscillations in the cooling curves.

Two horizontal thermocouple arrays were also utilized during MDS $1 \mathrm{R}$, and the results are shown in Figure 5.26. Results from both arrays suggested the existence of complete mixing in the melt throughout the experiment. In addition, temperatures dropped monotonically indicating the absence of double-diffusive convection cells. Early in the experiment, temperature fluctuations along the side wall mushy region were as large as $4^{\circ} \mathrm{C}$, indicating that thermal boundary layers along the side wall were disturbed by the rocking motion. However, the transitional lull in temperature fluctuations also appeared in data obtained from the horizontal thermocouple arrays, and later in the experiment, movement of the water-rich pool which collected at the top of the cavity restored temperature oscillations in the data.

A comparison of the melt temperatures recorded for the static and rocking cases was made to determine the effect of rocking on mixing within the melt (Fig. 5.27). Though providing very good mixing within the melt, temperatures with rocking corresponded approximately to a bulk average of temperature in the static melt. Therefore, the results show that, although the rocking motion inhibits stratification due to the formation of double-diffusive convection cells, overall heat transfer from the melt is not enhanced by the low rocking rates. This finding was verified numerically by a simulation of flow in a differentially heated two-dimensional box without solidification.

Temperature histories from case MDS $2 R$, where the rocking amplitude was changed to $30^{\circ}$ showed similar trends to those of experiment MDS 1R. However, a larger stratification of melt temperatures was present with the smaller rocking amplitude (Fig. 5.28). Although temperature fluctuations were initially as high as $3^{\circ} \mathrm{C}$, these fluctuations decreased and the inverted thermal stratification developed in the melt toward the later stages of the experiment. This stratification pattern was consistent with results for the 


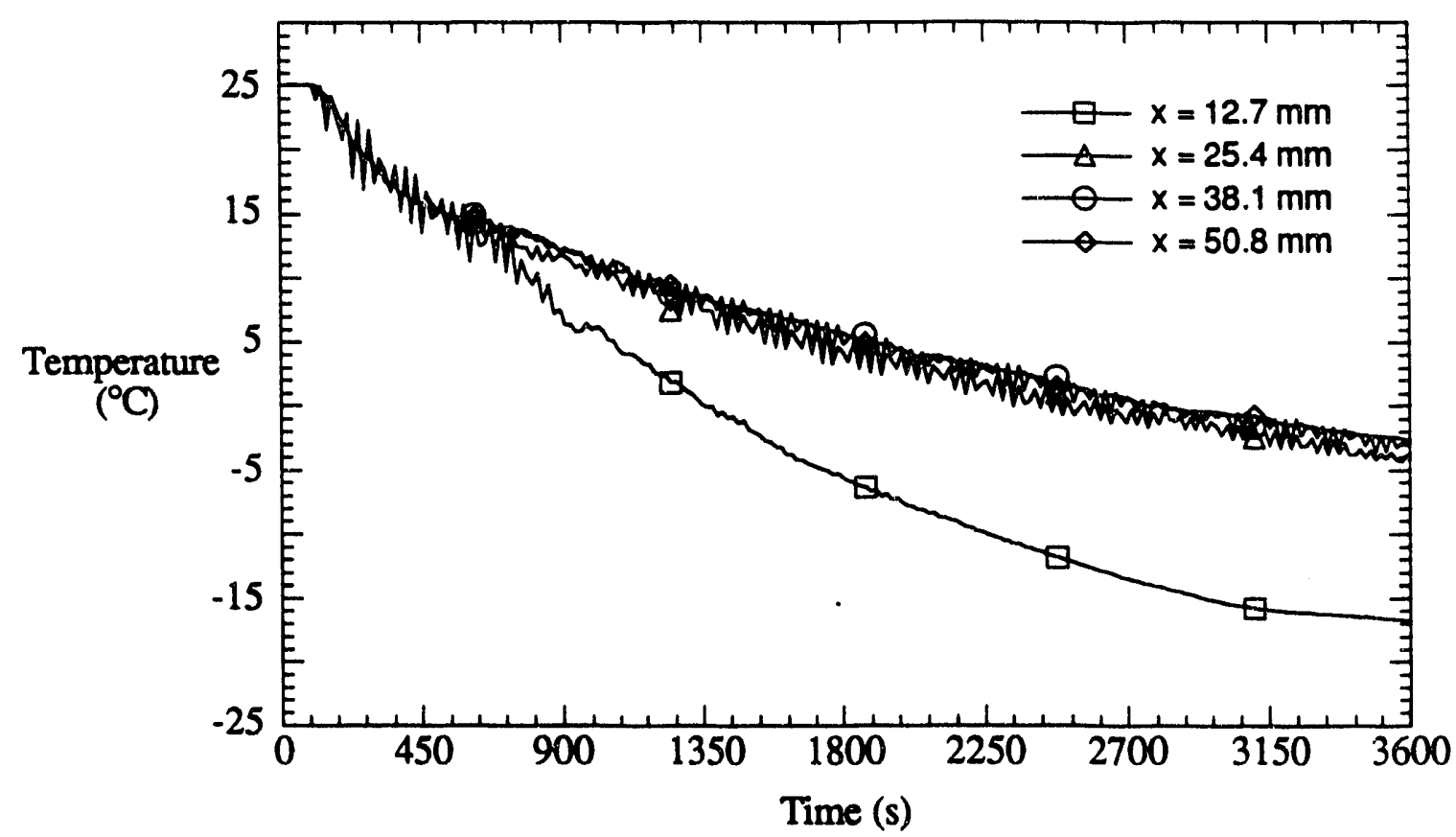

(a) Upper Array

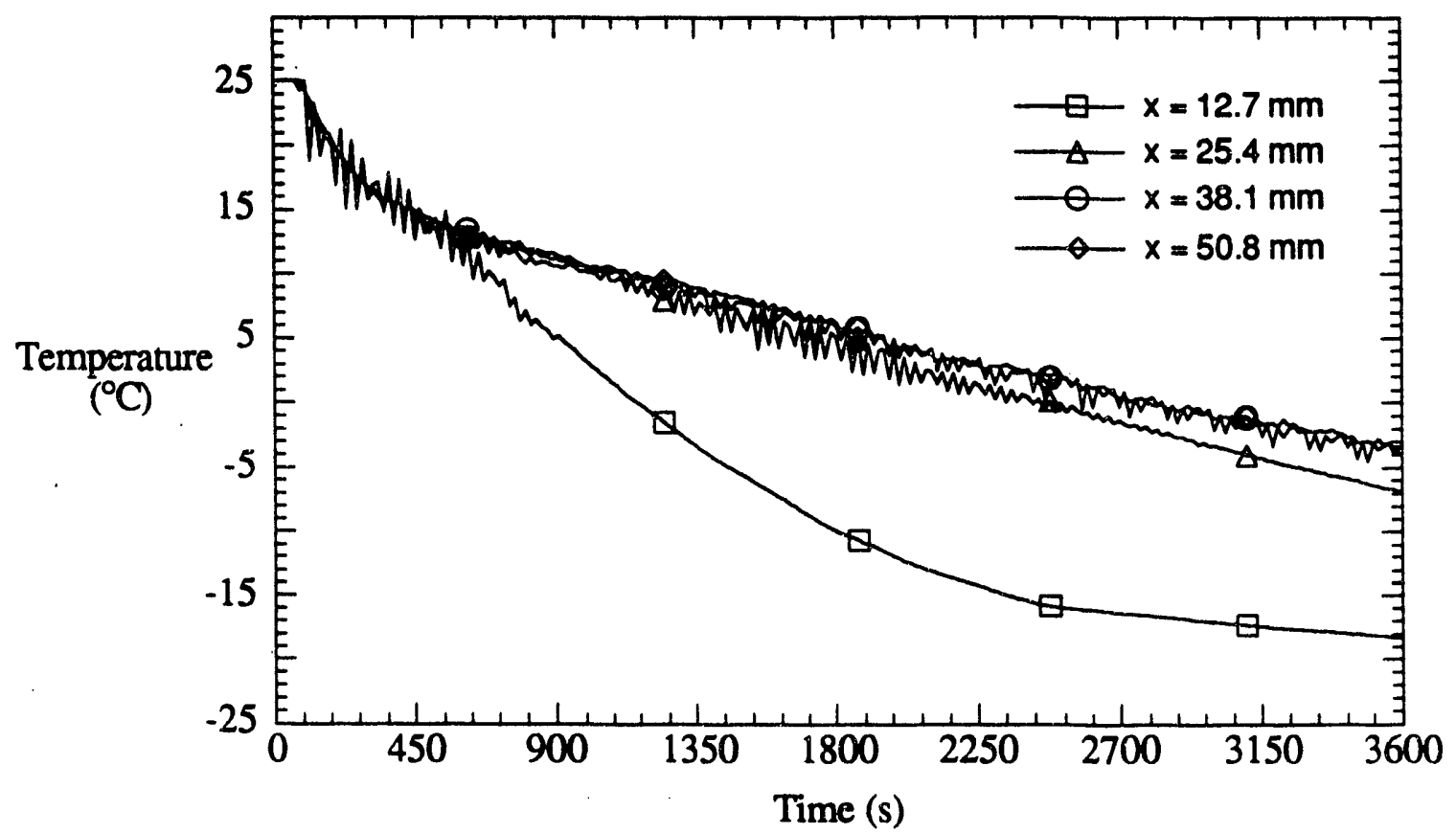

(b) Lower Array

Figure 5.26 Temperature histories for experiment MDS $1 \mathrm{R}$ at (a) $\mathrm{y}=76.2 \mathrm{~mm}$ and (b) $\mathrm{y}=50.8 \mathrm{~mm}$. 


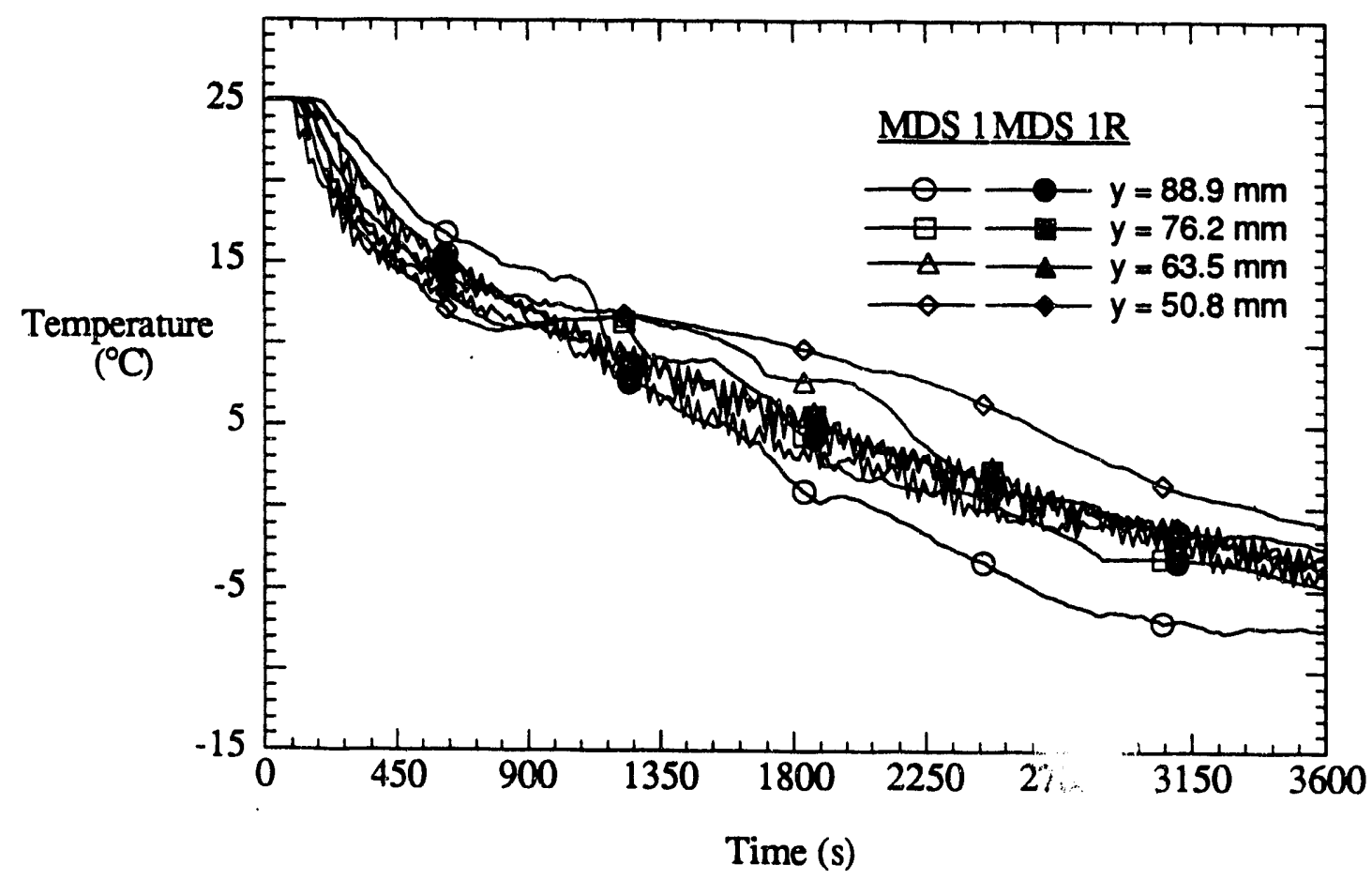

Figure 5.27 Comparison of melt temperatures for static and rocking experiments (MDS 1 and MDS 1R). 


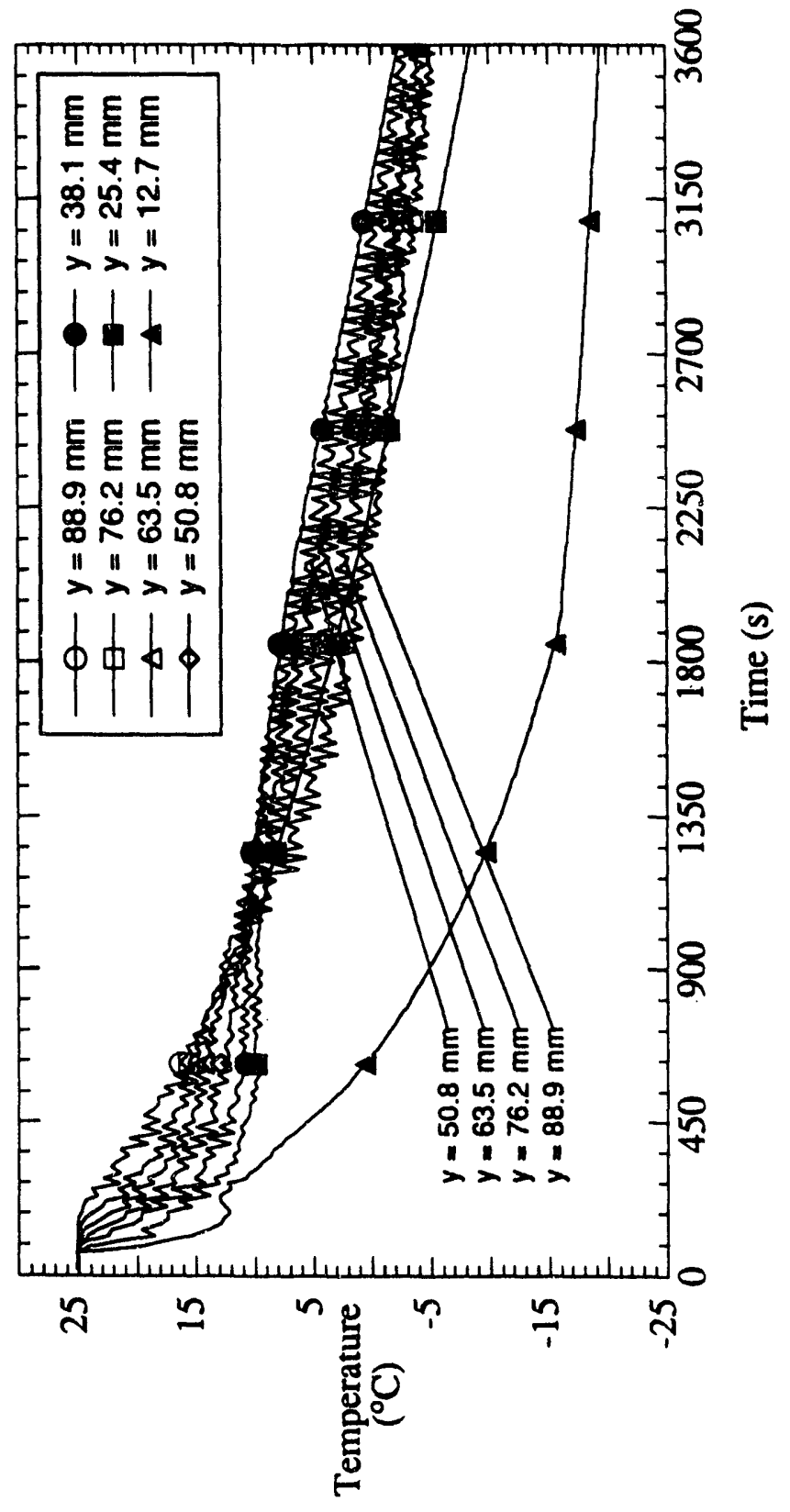

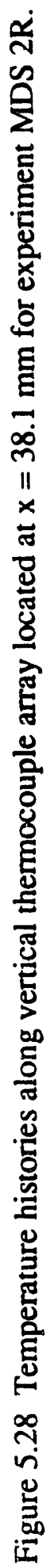


static experiments and was relatively large owing to the reduced inertial convection. However, the bulk melt temperature of MDS $2 \mathrm{R}$ differed little from that of MDS $1 \mathrm{R}$, both being about $-3^{\circ} \mathrm{C}$ after one hour. This observation further supports earlier findings that although it can efficiently mix fluid within the melt, the slow rocking motion does not enhance total heat transfer to the heat exchangers. Recalescence was again observed at the lowest thermocouple location (Fig. 5.28), but due to reduced liquidus sweeping, it was less severe than for case MDS $1 R$.

The temperature histories for the horizontal thermocouple arrays are shown in Figure 5.29, and these values were again, very similar to results from MDS 1R. Temperature fluctuations near the liquidus interface were large during the early stages of the experiment, but a lull in temperature fluctuations developed around 15 minutes as vertical temperature gradients in the melt decreased during transition from thermally dominant buoyancy to solutally dominant buoyancy. Beyond 22 minutes, the fluctuations in temperature returned as the cold, water-rich layer formed across the top of the cavity. Pushed by the rocking motion, this layer would oscillate across the top of the test section dropping the temperature at any thermocouple location with which it came into contact. This behavior can clearly be seen in the bottom thermocouple array (Fig. 5.29b). During the late stages of the experiment, only two thermocouples in the lower array remained in the melt $(x=38.1$ and $50.8 \mathrm{~mm})$, and the temperature at the center of the melt $(x=50.8$ $\mathrm{mm}$ ) dropped monotonically with little or no fluctuation. However, the outer temperature $(x=38.1 \mathrm{~mm})$ fluctuated by as much as $2^{\circ} \mathrm{C}$ as the cold, water-rich layer oscillated to that location twice every minute.

Results for experiment MDS 3R, with a rocking amplitude of $15^{\circ}$ (Figs. 5.30 and 5.31), began to approach the thermal characteristics of the static solidification runs (Figs. 4.21 and 4.22). In fact, experiment MDS $3 R$ was more similar to MDS 1 than to MDS 1R. Temperature histories recorded by the vertical thermocouple array became highly 


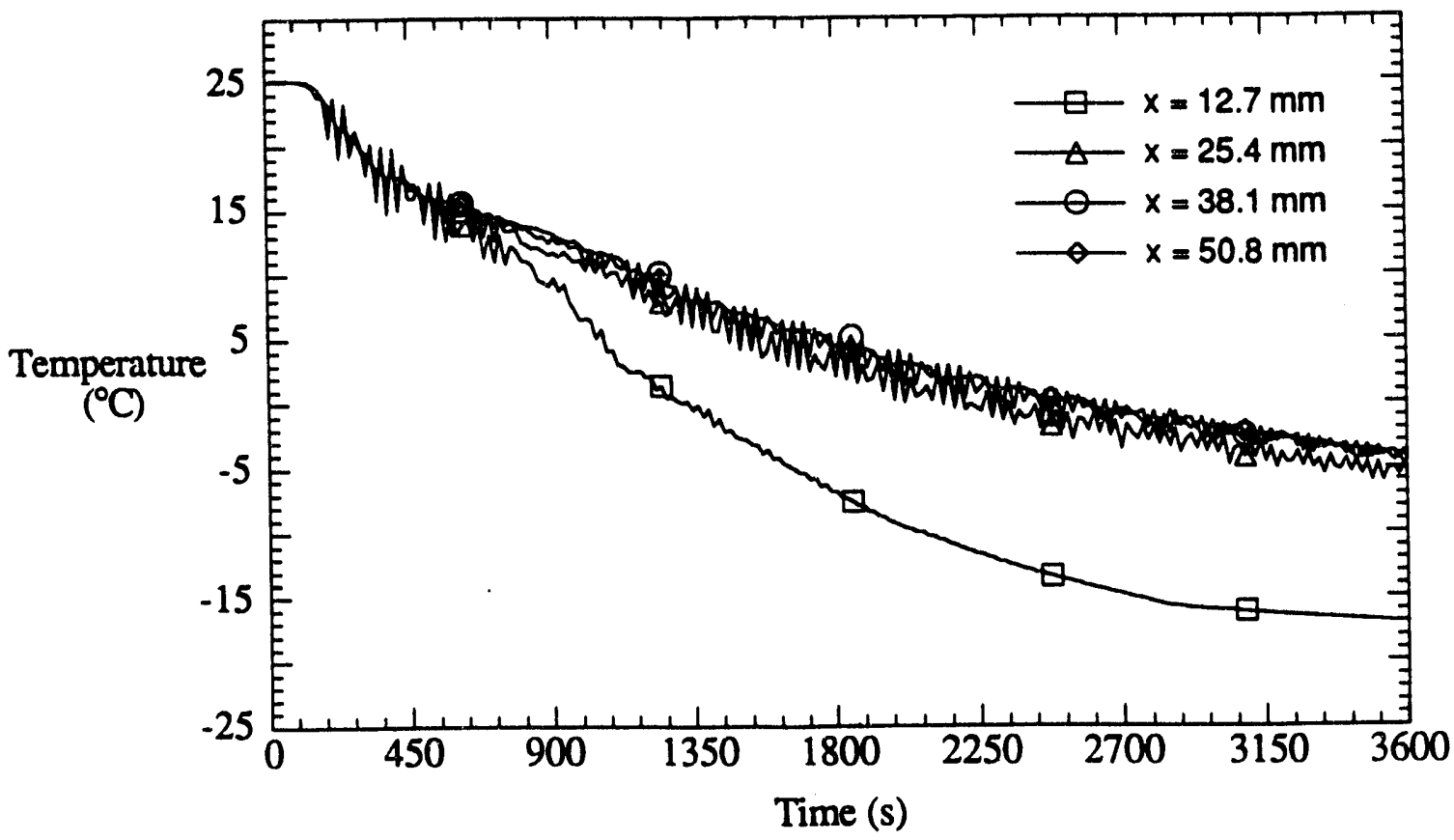

(a) Upper Array

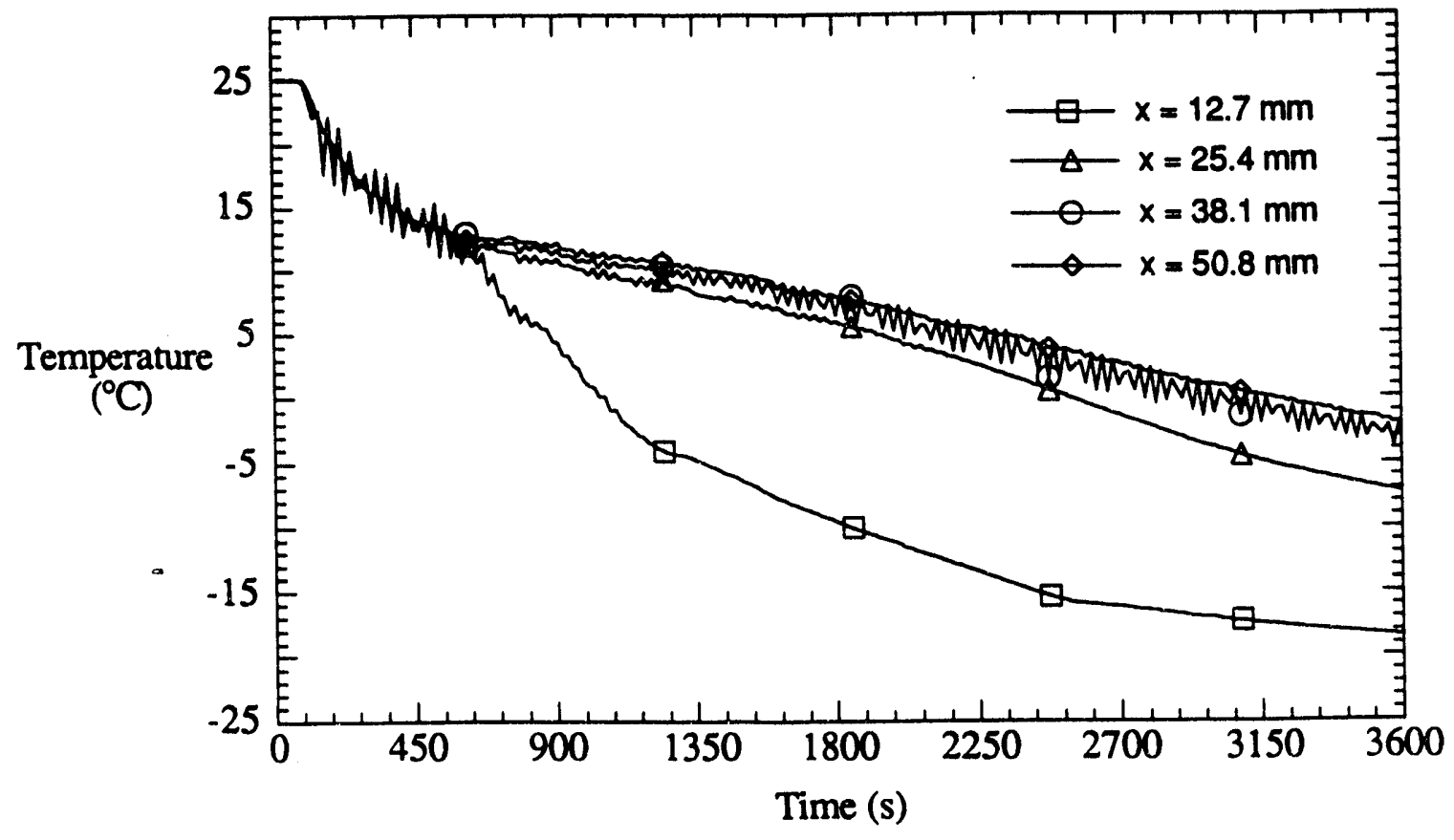

(b) Lower Array

Figure 5.29 Temperature histories for experiment MDS $2 R$ at (a) $y=76.2 \mathrm{~mm}$ and (b) $y=50.8 \mathrm{~mm}$. 

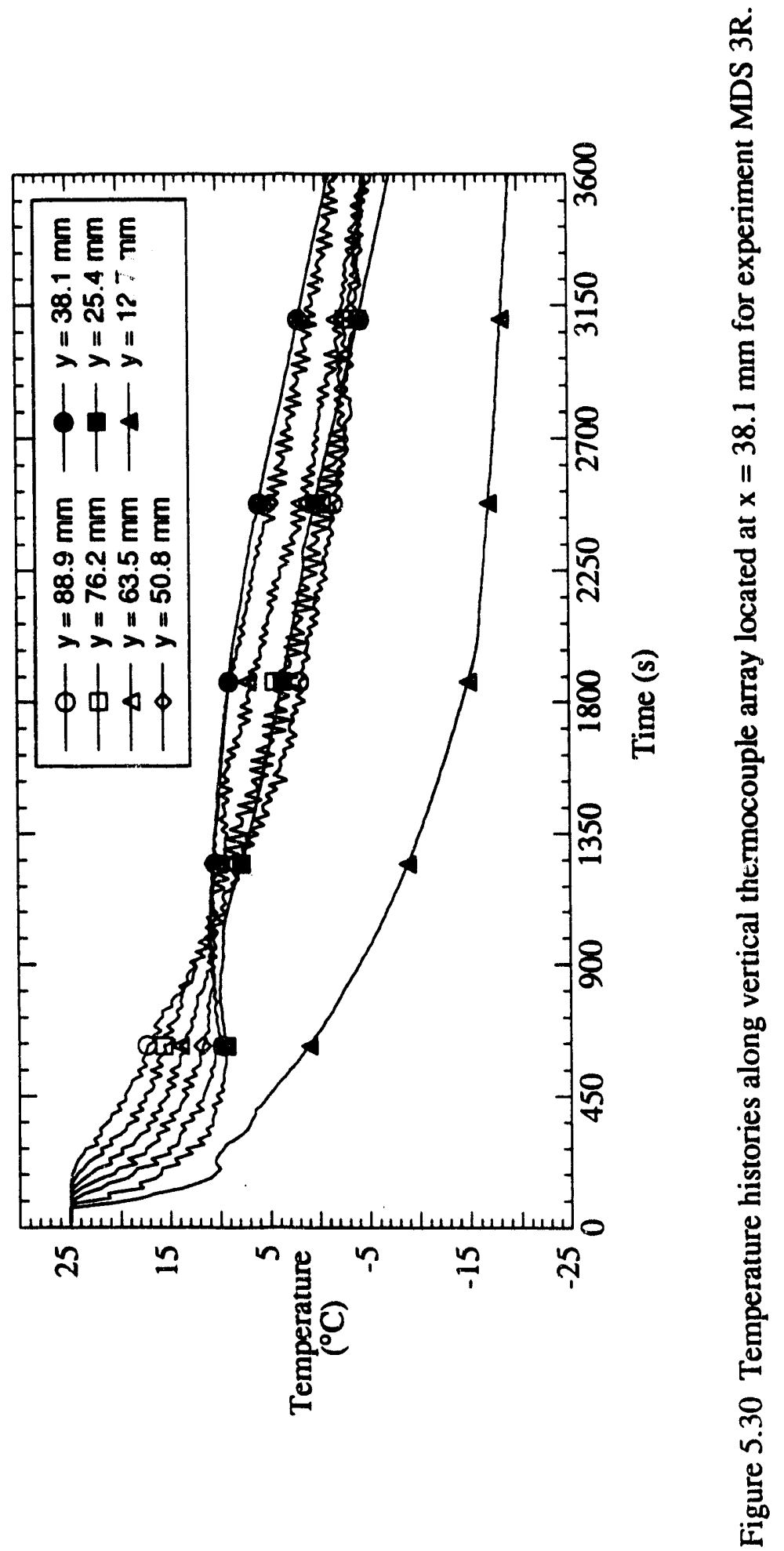


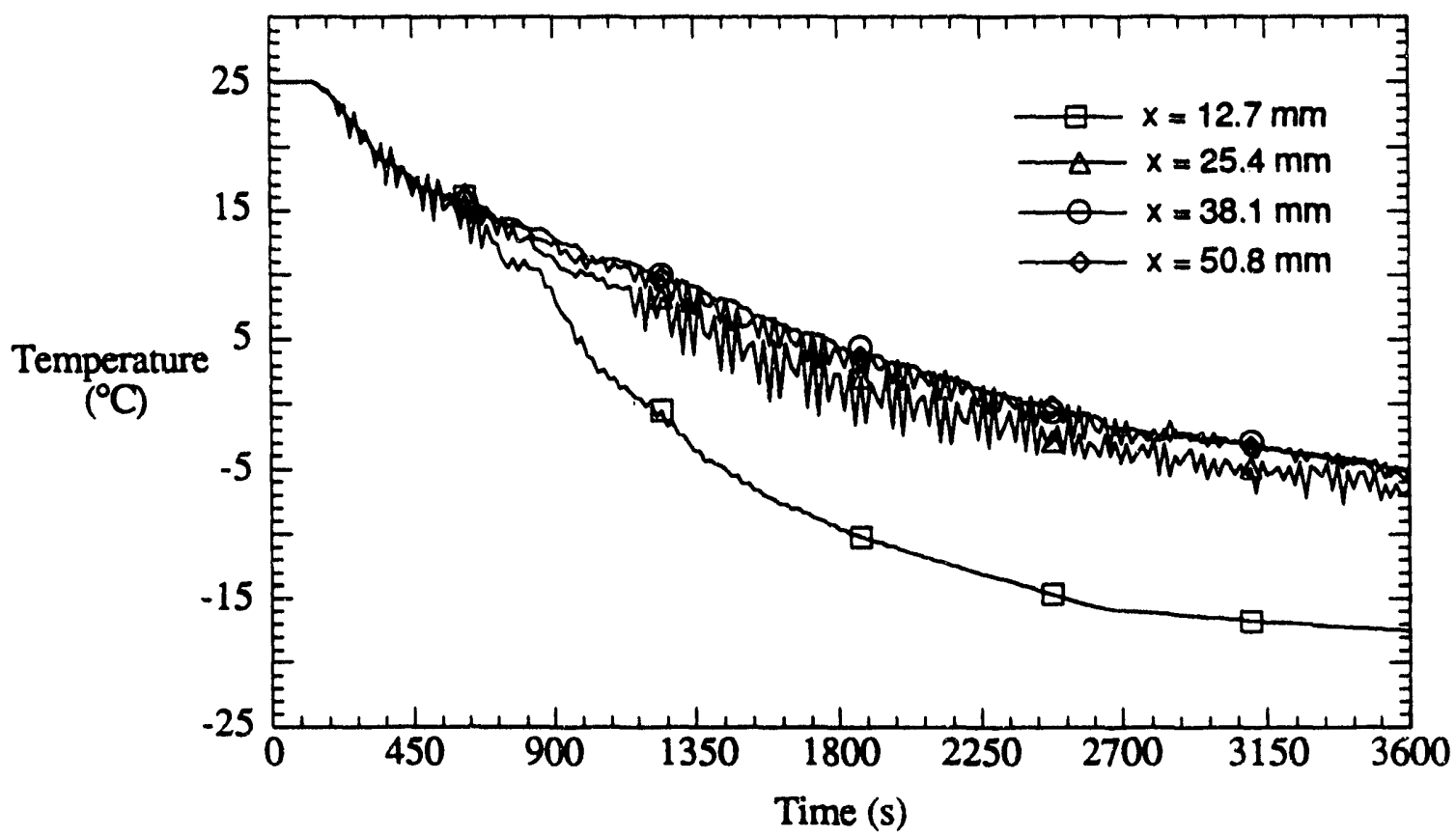

(a) Upper Array

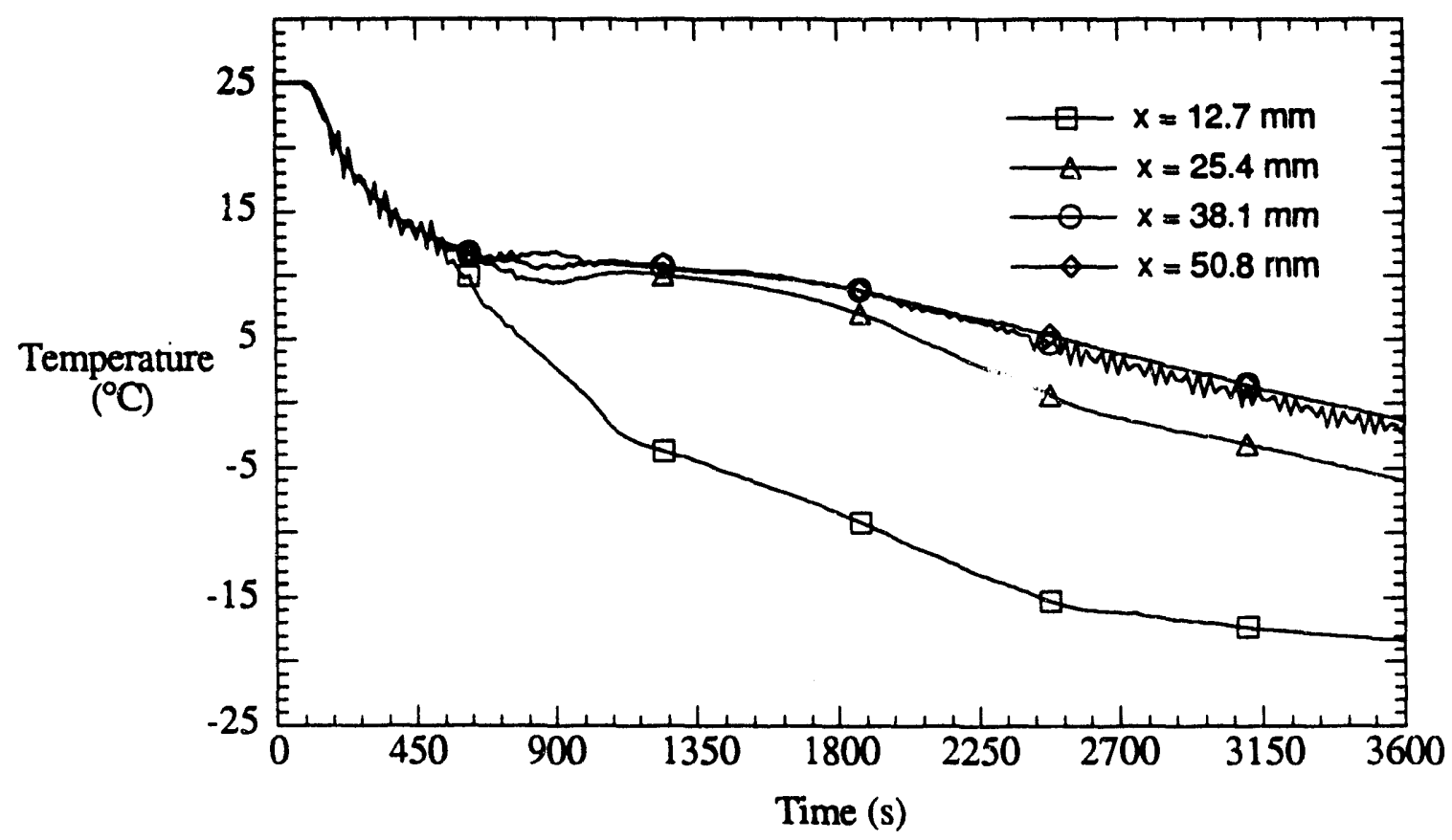

(b) Lower Array

Figure 5.31 Temperature histories for experiment $\operatorname{MDS} 3 \mathrm{R}$ at (a) $\mathrm{y}=76.2 \mathrm{~mm}$ and (b) y $=50.8 \mathrm{~mm}$. 
stratified beyond 20 minutes, indicating establishment of a water-rich cold pool in the upper regions of the cavity. This pool created strong oscillations, as high as $5^{\circ} \mathrm{C}$, at the outer thermocoupla in the upper horizontal array, as the rocking motion forced the pool to oscillate across the thermocouple location $(x=38.1 \mathrm{~mm})$.

An attempt was made to quantitatively describe temperature fluctuations in the melt for all MDS cases. Specifically, standard deviations of the actual data from linear curve tits of the data were determined (See Section 5.2.2). To ensure that meaningful comparisons could be made between temperature fluctuations in the melt for the rocking and static cases, three thermocouple positions on the upper array using 29 data points at a time period from 1900 to 2350 seconds were chosen. In the static case, these positions experienced the largest temperature oscillations, while avoiding any temperature steps associated with the passage of a double-diffusive interface. These positions were therefore amenable to analysis. The results of the analysis show that temperature fluctuations were relatively small for static solidification (Fig. 5.32). In contrast, the fluctuations were extremely strong for case MDS $3 R$, where a very cold pool of waterrich fluid collected at the top of the test section. Although slight rocking did not effectively mix the pool, it did cause it to oscillate back and forth across the cavity, thereby inducing a significant temperature change in those thermocouples which it crossed. On the other hand, rocking with larger amplitudes enhanced mixing of the solutal pool, thereby decreasing the fluctuations which it produced.

In comparing the overall melt temperature of the MDS experiments, little difference in the bulk temperature was observed. As shown in Figure 5.27, the rocking motion of MDS IR acted to mix and decrease temperature differences within the melt; however, the overall bulk temperature was uneffected. Similar trends were observed for the remaining rocking experiments. Although the melt became more stratified as the 


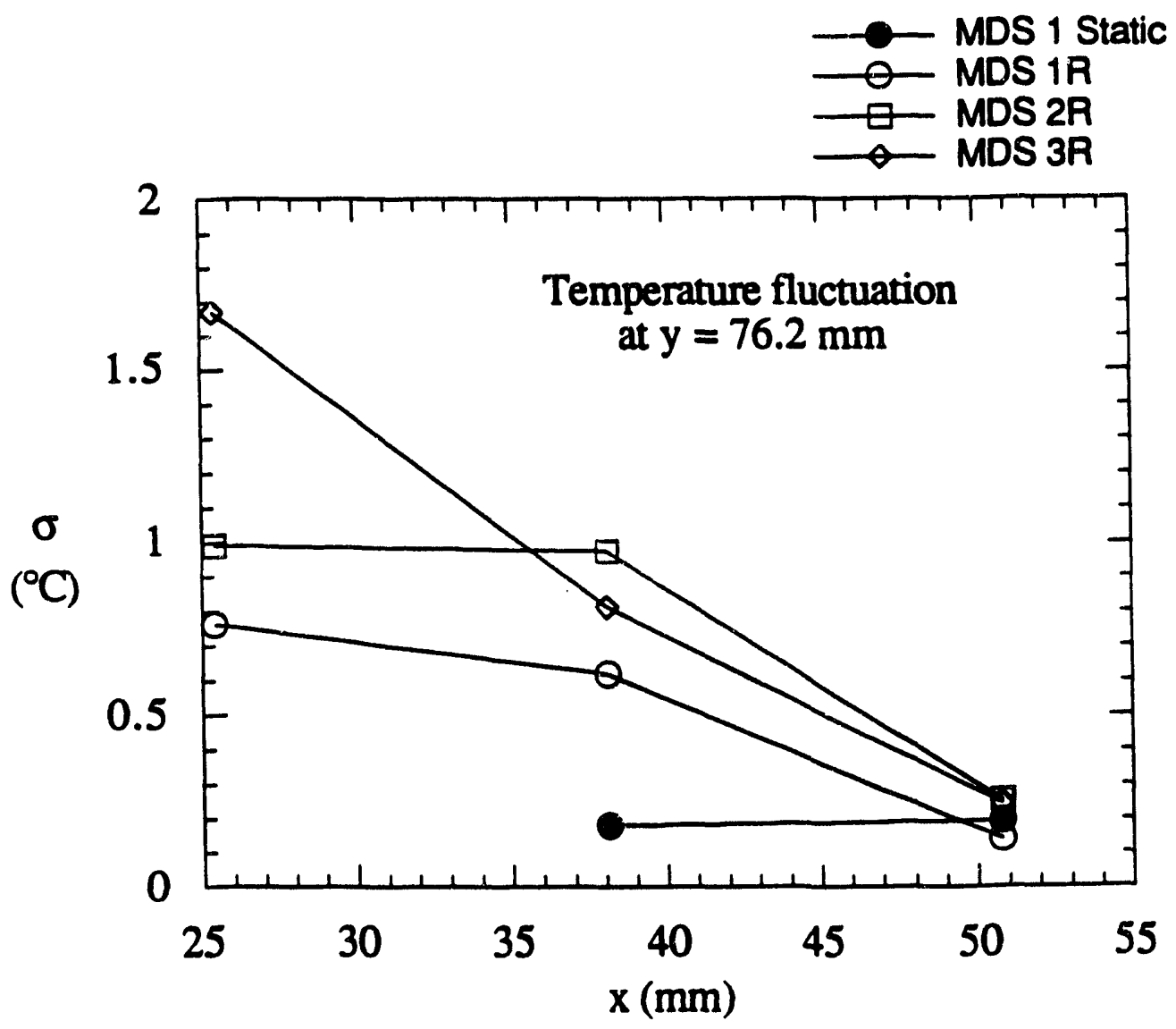

Figure 5.32 Standard deviations of temperature fluctuations in the melt for multidirectional solidification from 1900 to $2350 \mathrm{~s}$. 
rocking amplitude decreased, average melt temperatures were unchanged from experiment to experiment.

Species composition measurements were also taken for the rocking cases, and the results are presented in Figure 5.33. Mixing was most efficient with the larger amplitude, and large solutal stratifications occurred with small amplitude rocking. Although melt mixing could be improved, overall macrosegregation was unaffected by rocking as the overall liquid composition profile of each experiment was unchanged with the degree of rocking. Therefore, although macrosegregation was generally improved with the aid of rocking in UDS experiments, no such advantaged could be perceived for test section II. 


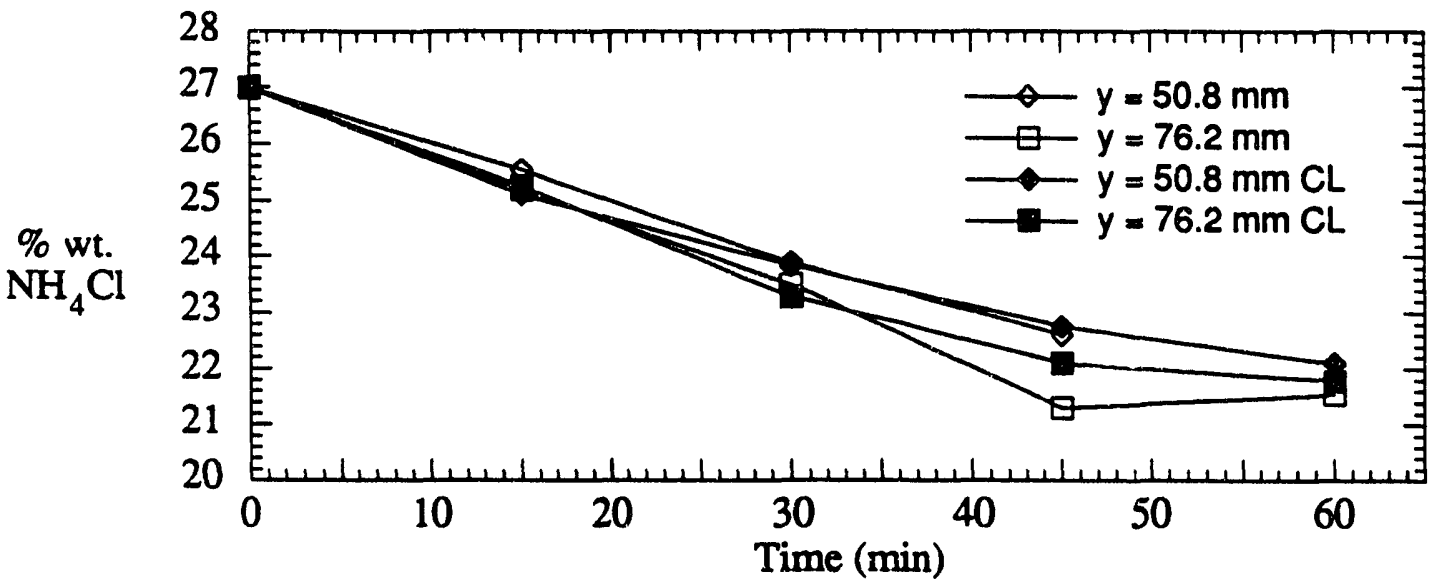

(a) MDS 1R

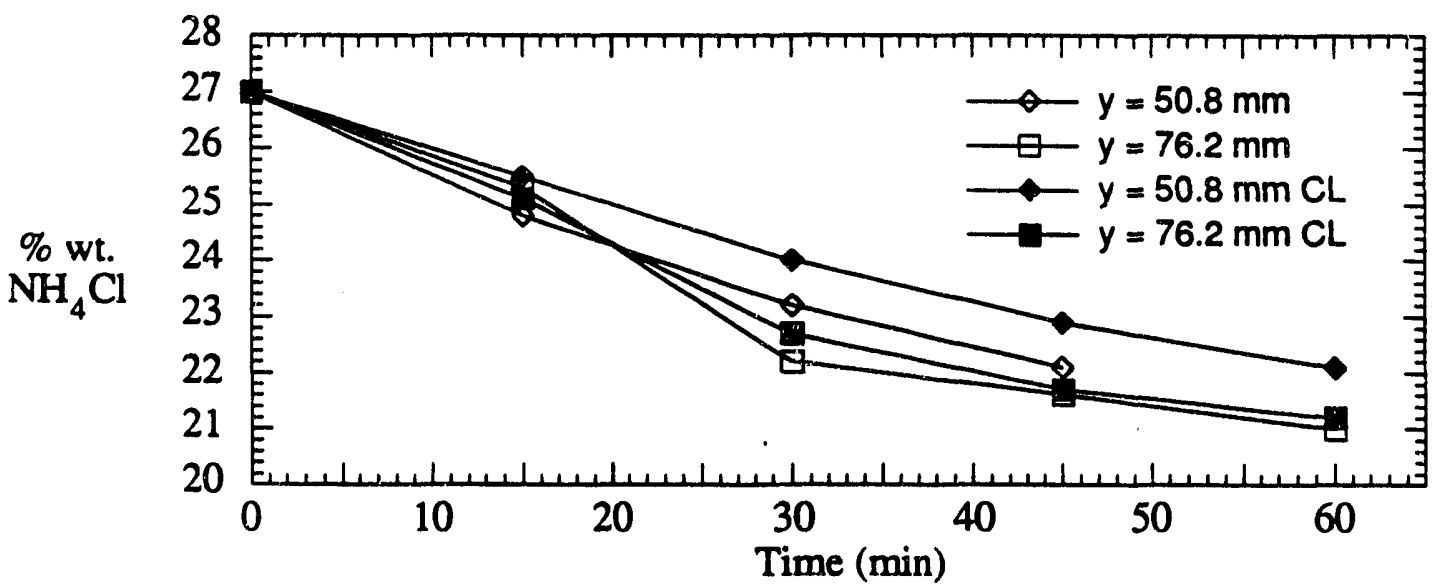

(b) MDS 2R

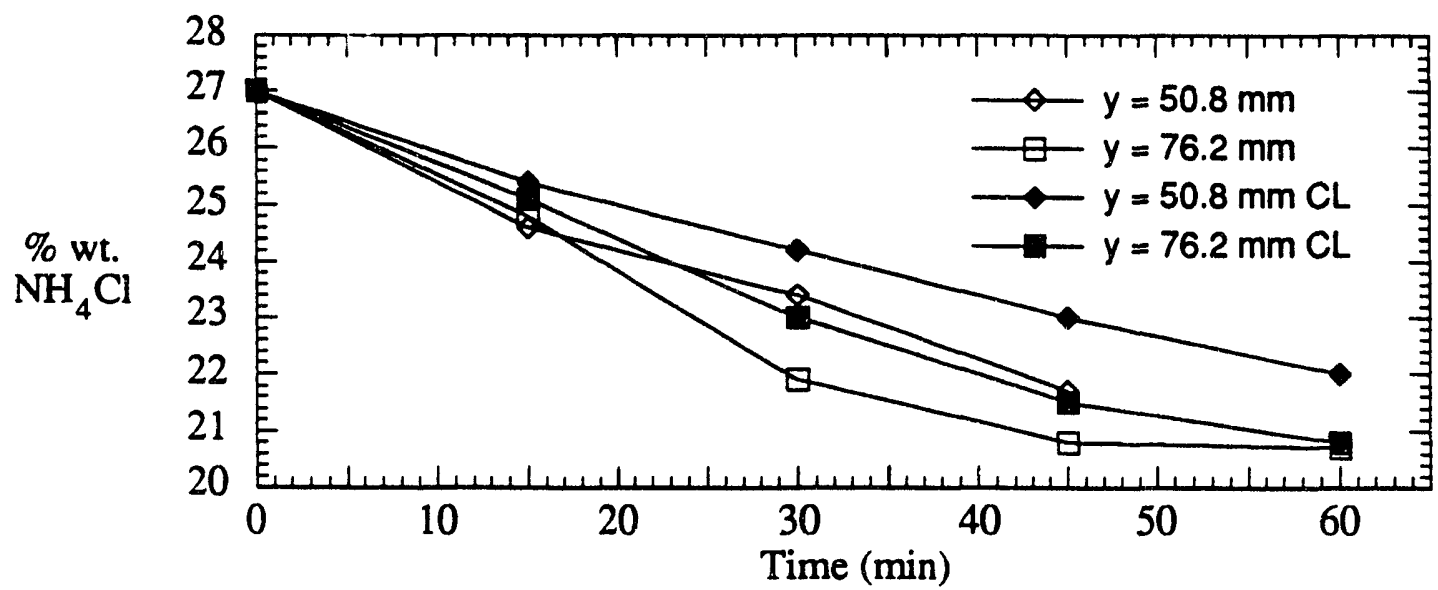

(c) MDS 3R

Figure 5.33 Liquid composition of ammonium chloride at $x=25.4 \mathrm{~mm}$ (open symbols) and at the centerline (closed symbols) for experiments (a) MDS 1R, (b) MDS 2R, and (c) MDS 3R. 


\section{CHAPTER 6 - NUMERICAL SIMULATIONS}

\subsection{Introduction}

Although a large amount of new and useful insights were revealed with the conventional experimental sechniques employed in the present study, a much larger body of important information eluded experimental examination. Therefore, the continuum model discussed in Chapter 3 was utilized to numerically simulate some key experiments. The numerical tool, a direct descendent of the model used by Neilson and Incropera (1991), possesses the capability to predict not only flow conditions, temperature, and composition fields within the melt, but also, to reveal relevant conditions within the mushy region as well as overall macrosegregation in the cast. Coupled with the experimental investigation, the numerical predictions provide additional insight helping to resolve the phenomena governing directional solidification of a binary mixture.

Three complete solidification simulations of experiments in test sections I and II were carried out. These included simulations of the static UDS experiment (UDS 1), the rocking UDS experiment with a rocking rate of one cycle per minute and a rocking amplitude of $45^{\circ}$ (UDS 4R), and the static side and bottom wall chilling experiment (MDS 1). The simulations were identified by NUDS 1 , NUDS $4 R$, and NMDS 1 , where the $N$ indicates numerical computation. In each case, a full 30 minutes of solidification were simulated, in which much care was taken to process and tabulate the important parameters at 15 second intervals. Although simulating only a small portion of the total casting process, the simulations do adequately investigate that critical solidification period early in the process when macrosegregation patterns are established (Bennon and Incropera, 1987b). In addition, the two UDS simulations (NUDS 1 and NUDS 4R) were extended to 
one hour. However, results from the additional 30 minutes of simulation were processed in a less labor intensive manner, neglecting most intermediate information and concentrating, instead, on the conditions at 60 minutes.

The properties of $\mathrm{NH}_{4} \mathrm{Cl}-\mathrm{H}_{2} \mathrm{O}$ used for this investigation (Table 6.1) are those used by Neilson and Incropera (1991). The value of the latent heat of fusion, $h_{f}$, is based on the eutectic composition because no relevant data exists for the range of compositions used in the present study. Working with solutions on the $\mathrm{NH}_{4} \mathrm{Cl}$ side of the eutectic, the liquidus temperature is given as

$$
\mathrm{T}_{\text {liq }}=(468.0) \mathrm{f}^{\mathrm{NH}} \mathrm{Cl}-107.7 \quad\left({ }^{\circ} \mathrm{C}\right)
$$

Because the crystals which form are pure $\mathrm{NH}_{4} \mathrm{Cl}$, the slope of the solidus line is assumed to be infinite.

The two-dimensional simulations were matched to the $101.6 \mathrm{~mm} \times 101.6 \mathrm{~mm}$ dimensions of the test sections using a Cartesian grid with 102 nodes in the $\mathrm{x}$-direction and 66 nodes in the y-direction (Fig. 6.1). The grid was weighted near the cold wall boundaries to improve resolution in the high thermal gradient regions such that more nodes are packed near the two side walls of test section II (Fig. 6.1b) than in the same region of test section I (Fig. 6.1a). The intersection of grid lines shown in Figure 6.1 defines the boundary of a particular control volume used in calculating most field variables. However, velocities were calculated on a staggered version of Figure 6.1.

The boundary and initial conditions of the simulations differed little between each case. Each boundary was treated with the no-slip condition; the respective heat exchanger surface was prescribed to be at a constant temperature, $\mathrm{T}_{\text {wall }}=-30^{\circ} \mathrm{C}$; and all other boundaries were treated as adiabatic and impermeable to species transfer. All simulations, as with the experiments, had a $27 \%$ wt. $\mathrm{NH}_{4} \mathrm{Cl}$ nominal composition and an initial temperature of $T_{\text {init }}=25^{\circ} \mathrm{C}$. In the static UDS case (NUDS 1), an initial random 
Table 6.1 Thermophysical properties of $\mathrm{NH}_{4} \mathrm{Cl}-\mathrm{H}_{2} \mathrm{O}$.

\begin{tabular}{|lcc|}
\hline & Solid & Liquid \\
Specific heat, $\mathrm{c}(\mathrm{J} / \mathrm{kg}-\mathrm{K})$ & 1870 & 3249 \\
Thermal Conductivity, $\mathrm{k}(\mathrm{W} / \mathrm{m}-\mathrm{K})$ & 2.7 & 0.468 \\
Density, $\rho\left(\mathrm{kg} / \mathrm{m}^{3}\right)$ & 1078 & 1078 \\
Diffusivity, $\mathrm{D}\left(\mathrm{m}^{2} / \mathrm{s}\right)$ & - & $4.8 \times 10^{-9}$ \\
Viscosity, $\mu(\mathrm{kg} / \mathrm{m}-\mathrm{s})$ & - & $1.3 \times 10^{-3}$ \\
Thermal expansion coefficient, $\beta_{\mathrm{T}}(1 / \mathrm{K})$ & - & $3.832 \times 10^{-4}$ \\
Solutal expansion coefficient, $\beta_{\mathrm{S}}\left(1 / \mathrm{f}_{l}^{\mathrm{NH}} \mathrm{Cl}\right)$ & - & -0.257 \\
& \multicolumn{2}{c|}{$3.138 \times 10^{5}$} \\
\hline Latent heat of fusion, $\mathrm{h}_{\mathrm{f}}(\mathrm{J} / \mathrm{kg})$ & \multicolumn{2}{c|}{$5.56 \times 10^{-12}$} \\
\hline Permeability constant, $\mathrm{K}_{0}\left(\mathrm{~m}^{2}\right)$ & \\
\hline
\end{tabular}




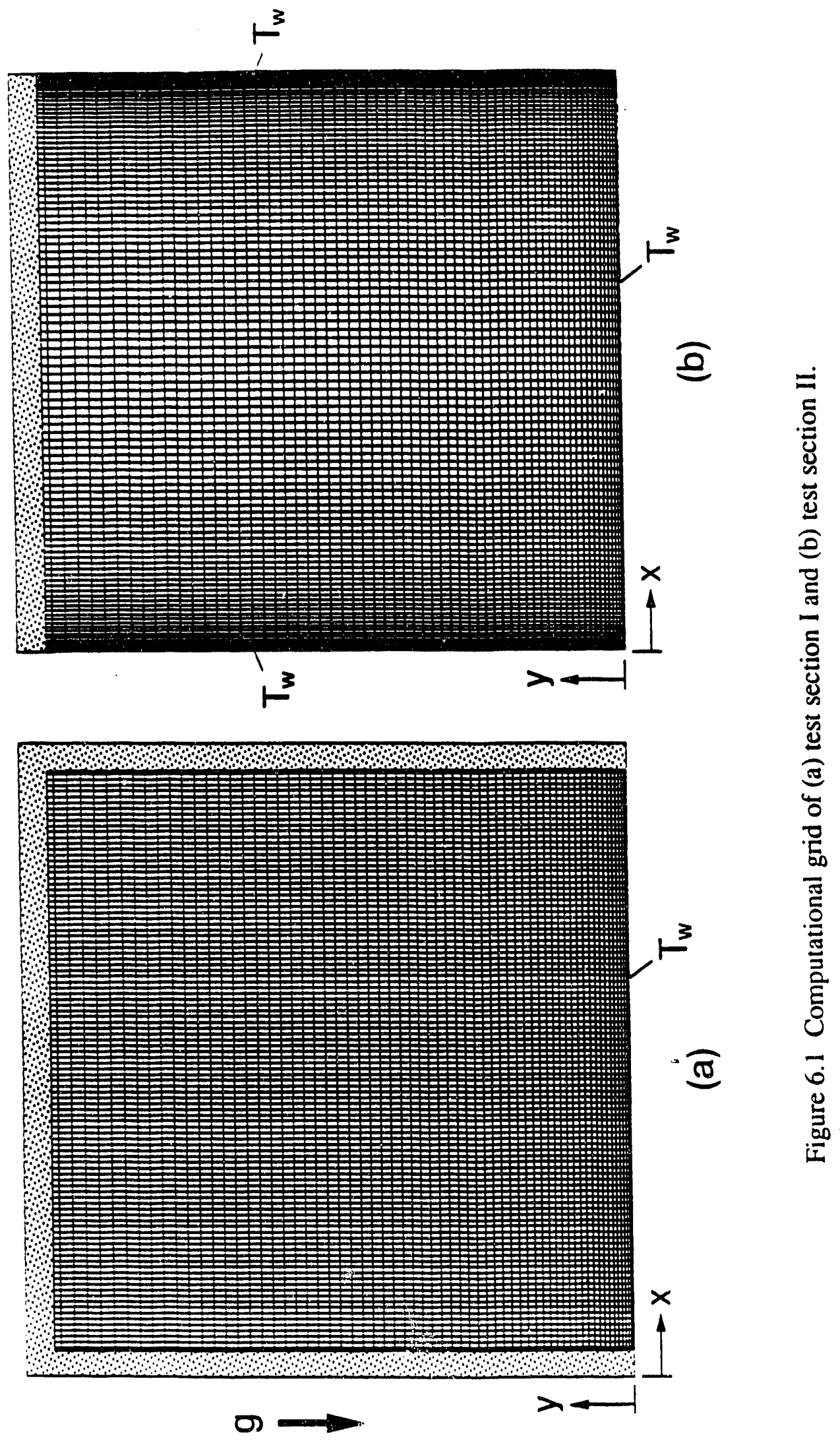


perturbation of $\Delta \mathrm{T}=0.01^{\circ} \mathrm{C}$ was applied to the temperature field so that nascent convection patterns in the melt would not be generated from numerical round-off error. Instead, the perturbations allowed convection patterns within the melt to develop randomly along the growth front in a manner congruent with experimental conditions. No such perturbations were necessary for the other cases. The rocking simulation was started at $\theta=-45^{\circ}$ from the vertical, where angular velocity is zero, and allowed to accelerate into its normal motion. For the first 15 seconds of NUDS $4 R$, from $\theta=-45^{\circ}$ to $\theta=0^{\circ}$, the cold wall temperature was prescribed to be $\mathrm{T}_{\text {wall }}=25^{\circ} \mathrm{C}$ (i.e. no solidification), but at 15 seconds, the cold wall was impulsively dropped to its intended chilled value of $T_{\text {wall }}=-30^{\circ} \mathrm{C}$.

The time step for the simulations was chosen to be $\Delta t=1 \mathrm{~s}$, and from 30 to 40 iterations were required to achieve convergence for a typical time step. The numerical method employed to solve the set of continuum equations required some slight relaxation. Relaxation coefficients of $0.9-1.0$ were used in solving the species and energy equations, and values of $0.8-0.9$ were used in solving the momentum and pressure correction equations for the UDS simulations. Much smaller relaxation coefficients of 0.6 - 0.7 were necessary to solve the momentum and pressure correction equations of case NMDS 1.

\subsection{Static Unidirectional Solidification}

The numerical simulation of experimental case UDS 1 is similar to previous investigations (Neilson and Incropera, 1991). The results of the simulation can be seen in Figures 6.2 - 6.6 .

The first five minutes of the simulation were dominated by one-dimensional conduction, during which both the mushy region and solid advanced into the melt. Other than small random bulk motions which were generated by the initial temperature perturbations, the melt was essentially quiescent. This prediction is in stark contrast to the actual experimental observations showing vigorous free convection in the form of salt 

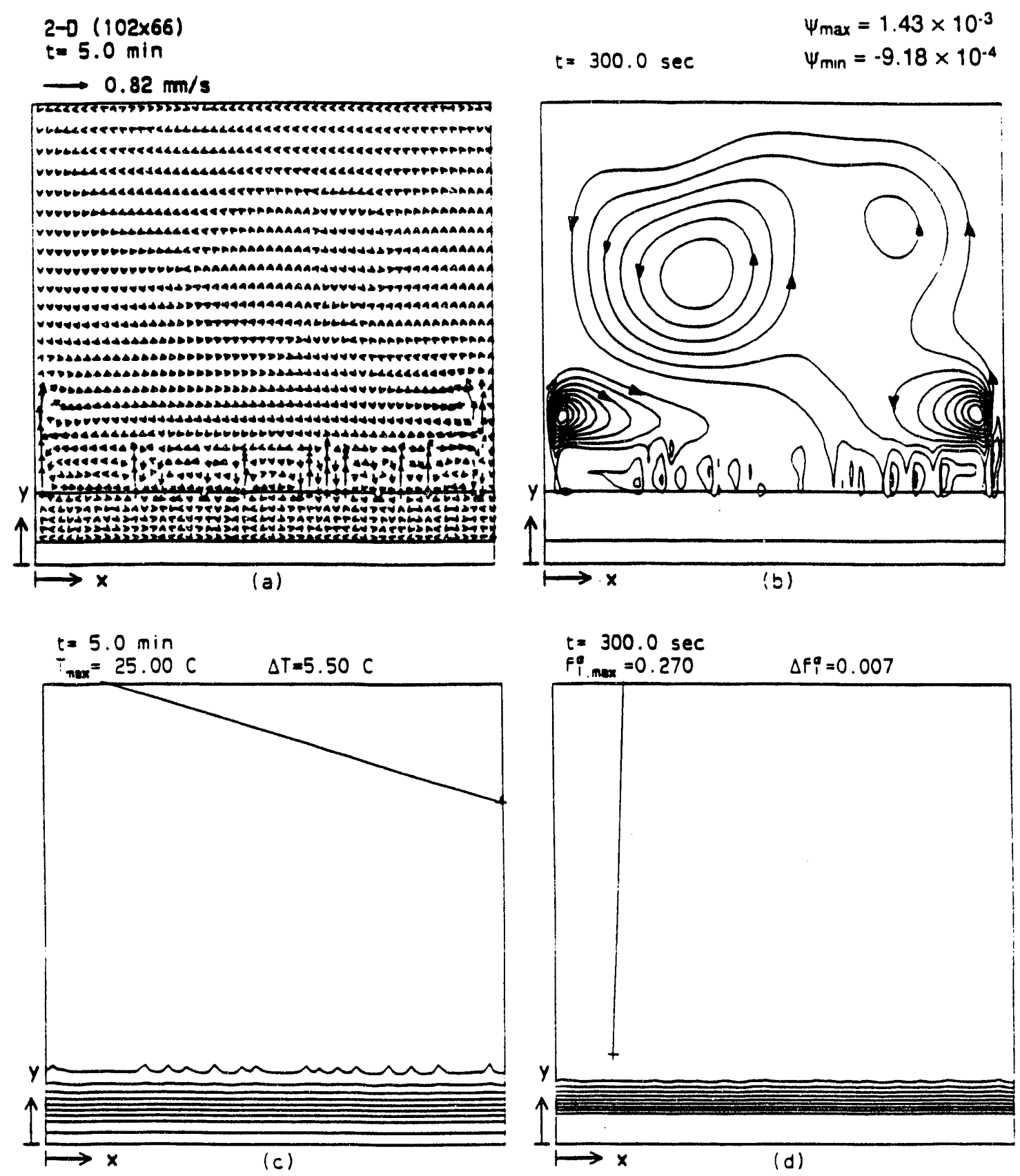

Figure 6.2 Predicted solidification behavior of case NUDS 1 at $300 \mathrm{~s}$ : (a) velocity vectors, (b) streamlines, (c) isotherms, and (d) liquid isocomposition. 


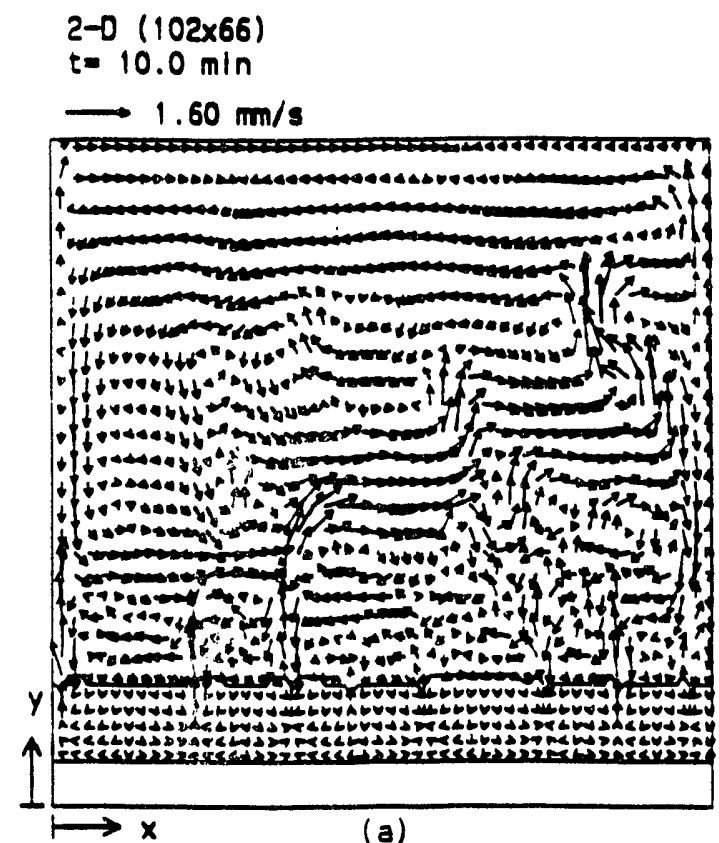

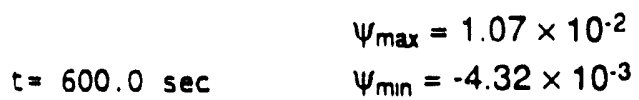
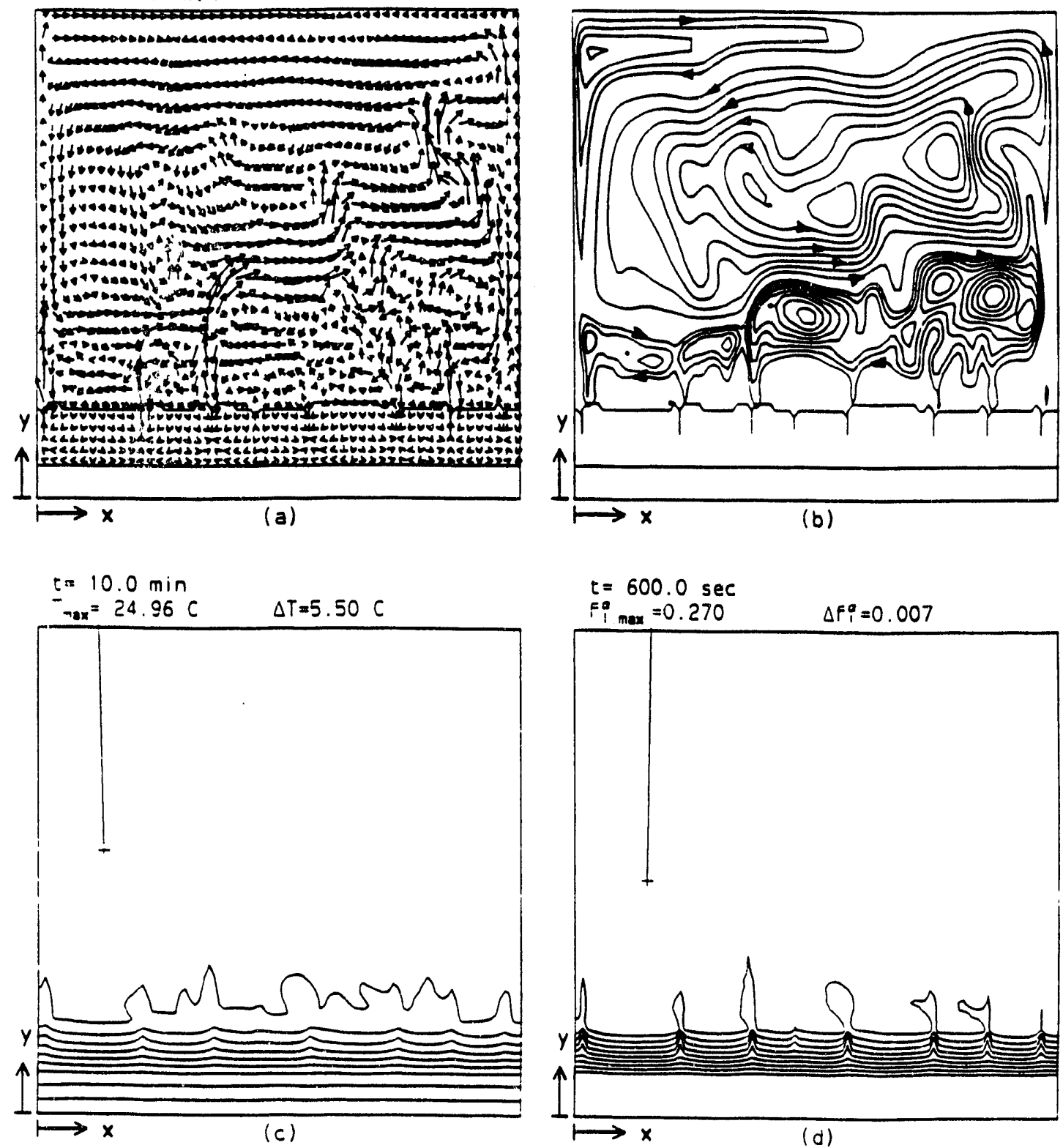

Figure 6.3 Predicted solidification behavior of case NUDS 1 at 600 s: (a) velocity vectors, (b) streamlines, (c) isotherms, and (d) liquid isocomposition. 

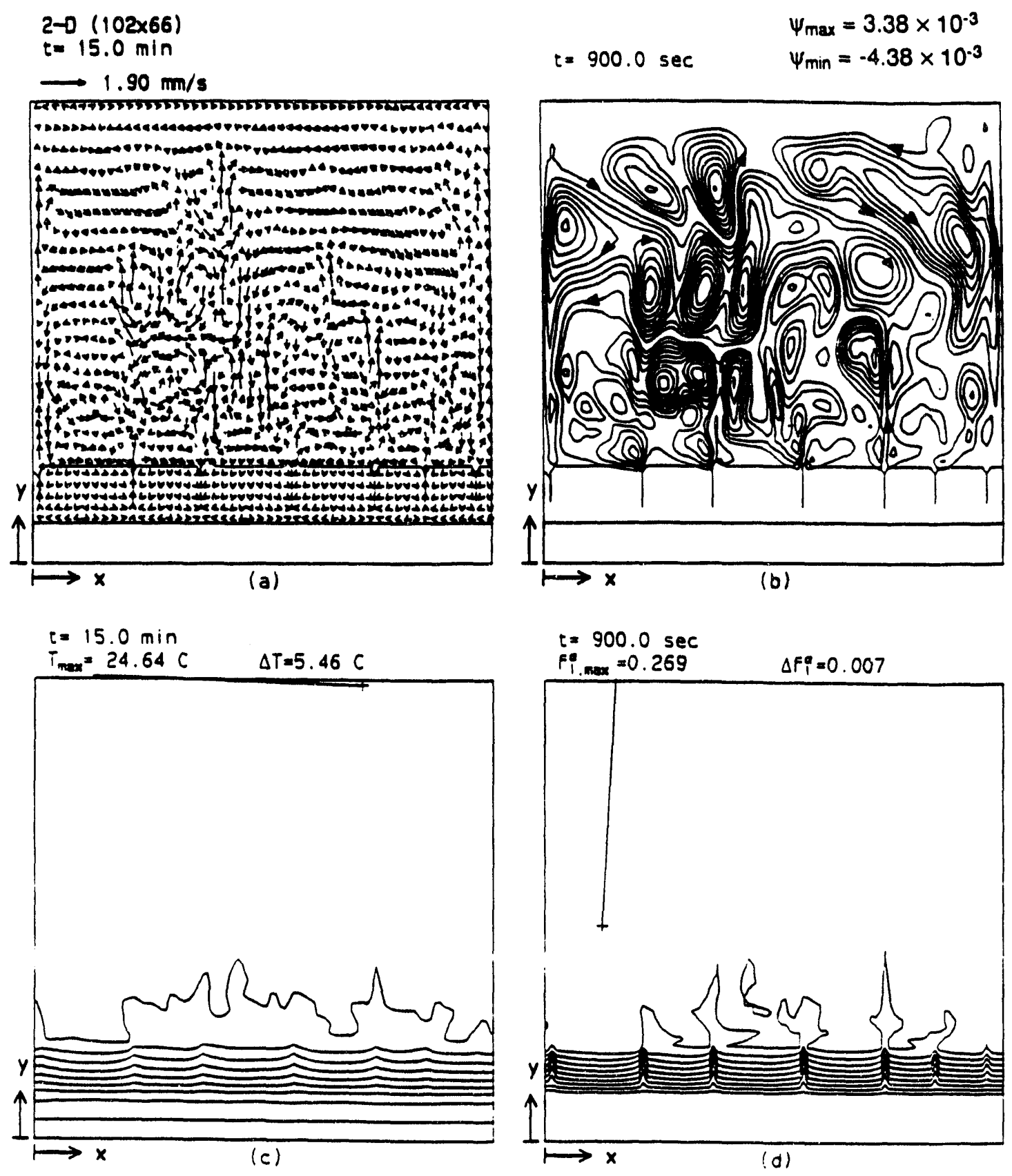

Figure 6.4 Predicted solidification behavior of case NUDS 1 at $900 \mathrm{~s}$ : (a) velocity vectors, (b) streamlines, (c) isotherms, and (d) liquid isocomposition. 

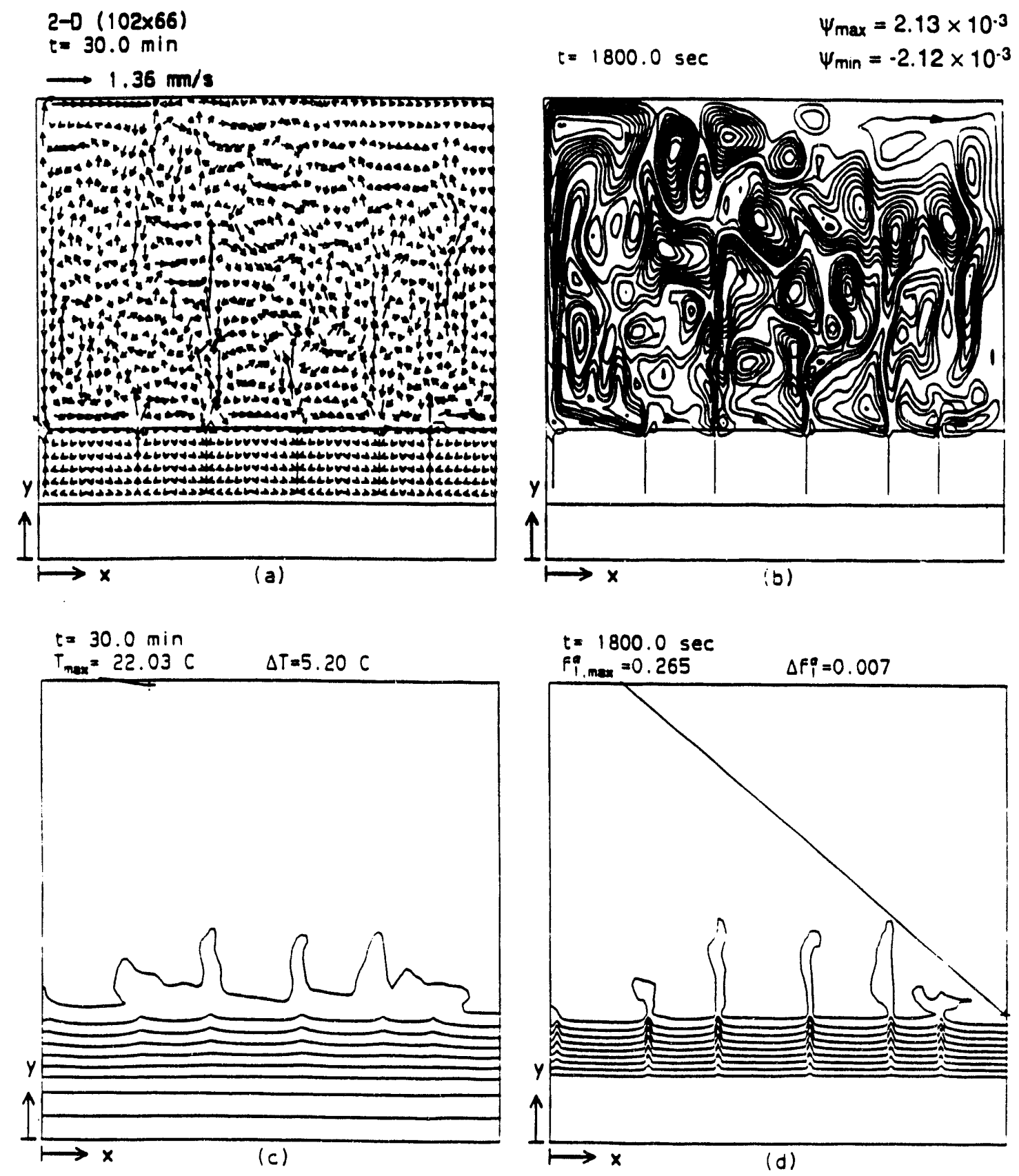

Figure 6.5 Predicted solidification behavior of case NUDS 1 at $1800 \mathrm{~s}$ : (a) velocity vectors, (b) streamlines, (c) isotherms, and (d) liquid isocomposition. 


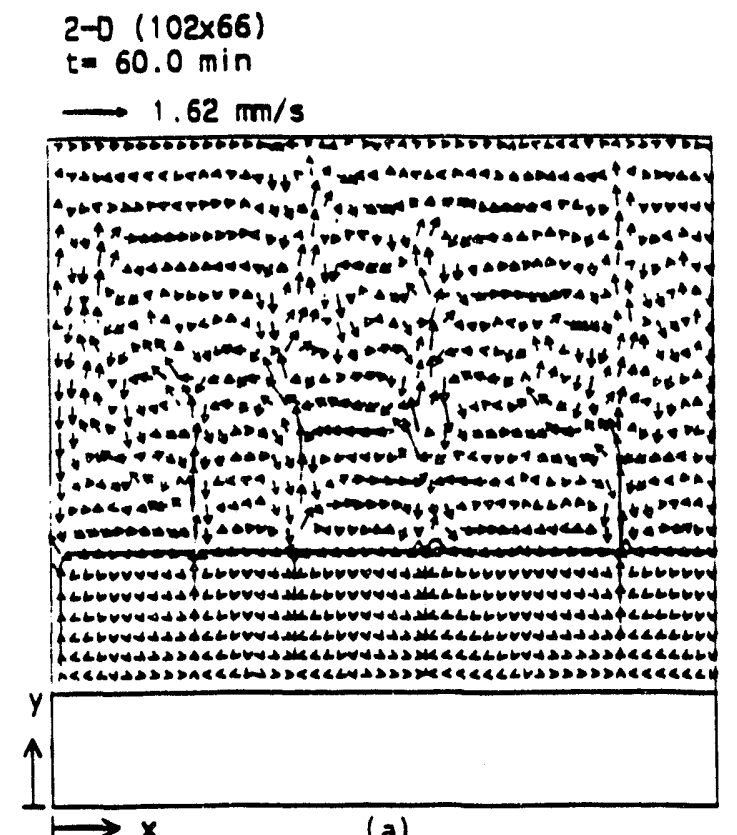

$$
\begin{aligned}
& \Psi_{\max }=1.83 \times 10.3 \\
& t=3600.0 \mathrm{sec}
\end{aligned}
$$
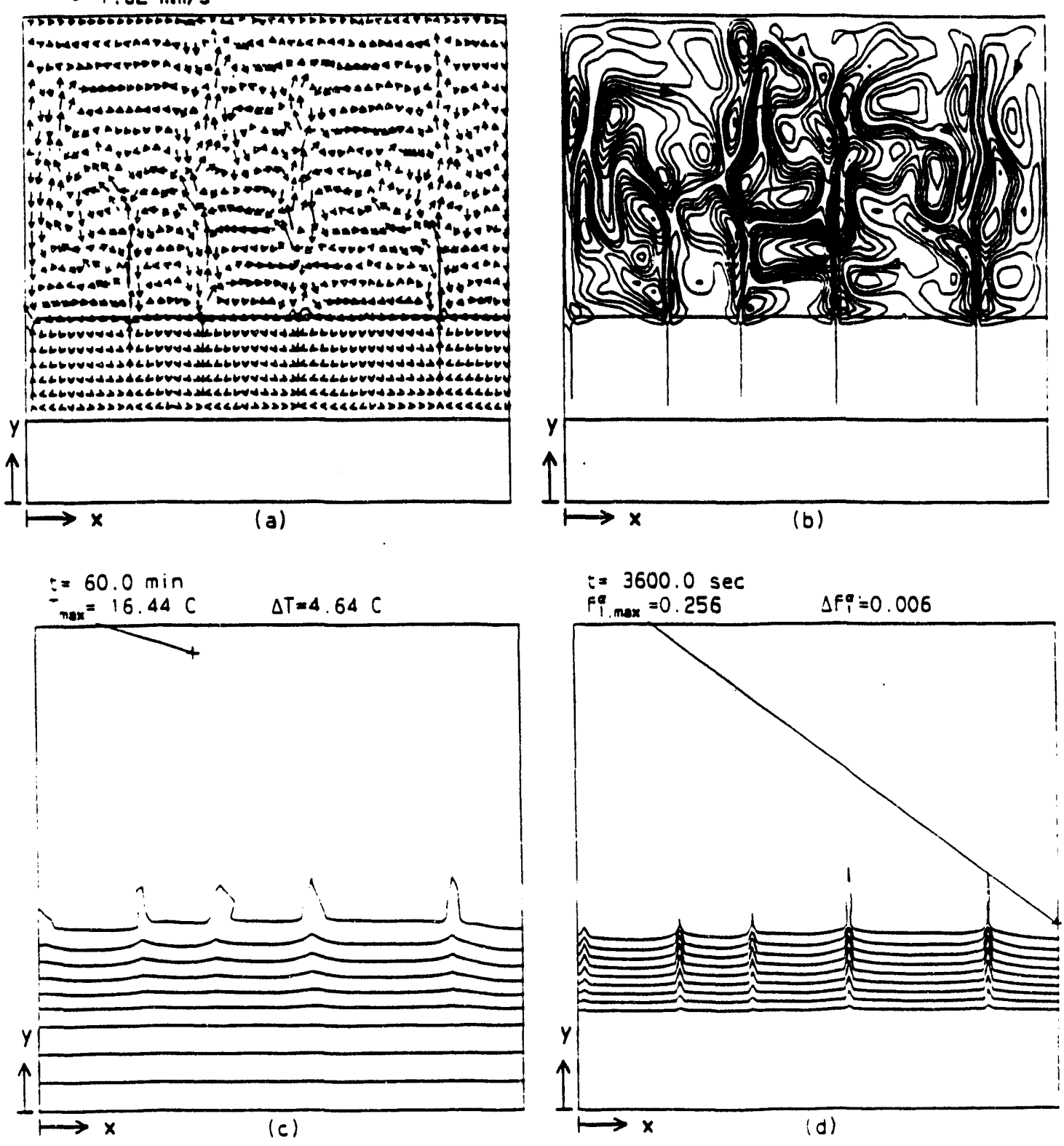

Figure 6.6 Predicted solidification behavior of case NUDS 1 at $3600 \mathrm{~s}$ : (a) velocity vectors, (b) streamlines, (c) isotherms, and (d) liquid isocomposition. 
fingering early in the experiment (Fig. 4.4). As the mushy region developed, it gradually rejected light, water-rich fluid thus creating an unstable density stratification. Shortly before five minutes, the unstable solutal buoyancy became stronger than both the stabilizing thermal and viscous forces present along the growth front, and free convection ensued. Located at random sites along the liquidus interface, strengthening buoyant parcels of fluid, about the size of a control volume, rose among falling negatively buoyant fluid (Fig. 6.2a and b). Resulting from the same mechanisms which produced salt fingering, the rising and falling parcels of fluid represent the model's best attempt to simulate the double-diffusive phenomenon. However, the control volumes of the existing grid were too large to fully and accurately capture the small scale transport associated with salt fingering. The temperature and composition fields were generally planar during the quiescent stage of the experiment, but small spikes, corresponding to incipient convection, had developed by five minutes (Fig. $6.2 \mathrm{c}$ and d). The units of $\psi$ are $\mathrm{kg} / \mathrm{s} \cdot \mathrm{m}$, and its maximum value is located in the lower right corner and its minimum value is located in the lower left comer (Fig. 6.21).

At ten minutes into the simulation, eight distinct channels were visible within the mushy region (Fig. 6.3). The channels, whose width corresponded to the size of a control volume, spewed water-rich fluid into the melt in the form of weak plumes, which induced random flow conditions in the melt (Fig. 6.3b). The locations of the plumes above the growth front can be clearly seen as sharp spikes in the isocomps, and although also visible in the isotherms, the channels and plumes appear less distinct on the temperature plots because thermal energy diffuses faster than species in the $\mathrm{NH}_{4} \mathrm{Cl}-\mathrm{H}_{2} \mathrm{O}$ mixture (Fig. 6.3c and d).

Fifteen minutes into the simulation, strong plumes issuing cold, water-rich fluid developed above deepening channels (Fig. 6.4). Also, the plumes strongly influenced flow conditions in the melt, as the streamlines had become more vertically aligned (Fig. 6.4b). The number of channels had dropped by one as the competition between channels 
to relieve the buoyancy potential of interdendritic fluid intensified. Flow within the mushy region was strongly influenced by the channels. Salt-rich fluid from the melt was drawn down into the mush at locations far from the channels, and once within the porous medium, the fluid was slowly entrained into the channels.

Conditions at 30 minutes were dominated by strong plumes issuing from six remaining channels (Fig. 6.5). The plumes were very robust, rising to the top of the melt, and the many vortices of rotating fluid which existed in the cavity illustrate the complex fluid motions driven by shear forces as well as by thermal and solutal buoyancy (Fig 6.5b). The plumes' influence on thermal conditions in the melt had just begun to be felt, as the peak temperature in the system was a relatively warm $22.03^{\circ} \mathrm{C}$ (Fig. 6.5c). Although fewer in number, the channels were very deep and appeared well developed. The thickening mushy region was generally uniform across the width of the test section, and interdendritic flow was still dominated by the channels. Evidence of the organized pattern of plumes can also be seen in the temperature and composition fields (Fig. 6.5c and d). Long vertical plumes of cold, water-rich fluid rose from the mushy region, generally undisturbed by flow conditions in the melt. As time progressed, the plumes grew even stronger and were less affected by bulk fluid motion in the melt.

Figure 6.6 shows conditions at one hour into the simulation. Only five channels remained, and those channels were very deep and ejected strong plumes. The plumes began to exhibit the same oscillatory behavior associated with plumes observed in experiments, but the predictiod plumes were much larger than those observed experimentally. While predicted plumes were from two to three control volumes wide, plumes observed in the experiments were about $1.5 \mathrm{~mm}$ in diameter, which corresponds approximately to the width of a control volume in the existing simulation. The present grid is therefore too coarse to capture the small scale intricacies associated with the plumes. At one hour, mixing induced by the plumes had effectively chilled the melt, dropping the peak 
temperature to $16.44^{\circ} \mathrm{C}$ (Fig. 6.6c). Thermal and solutal gradients in the mushy region were relatively weak, indicating the diminishing buoyant potential in the mush. Moreover, tive strength and maturity of the plumes suggested the establishment of severe macrosegregation in and around the channels. Macrosegregation plots support this premise.

Figure 6.7 shows the severity and location of macrosegregation for case NUDS 1 at 30 and 60 minutes. The value of macrosegregation is calculated as the total mass fraction of $\mathrm{NH}_{4} \mathrm{Cl}$ contained within all phases, solid and liquid, of a control volume. Similar to the macrosegregation profiles of Neilson and Incropera (1992a), macrosegregation in the cast was promoted by channels. Both the maximum and minimum values of $\mathrm{NH}_{4} \mathrm{Cl}$ composition were found near a channel, as fluid within the channel was nearly eutectic, while a salt-rich sheath of crystals grew around the channel. It should be noted that the black areas at the channel locations are not shading, but a congregation of macrosegregation contours produced by steep composition gradients in the vicinity of the channels. Both extremes were caused by the same process. As near nominal composition fluid was drawn into the mushy region by entrainment to the channels (Fig. 6.6a), it froze $\mathrm{NH}_{4} \mathrm{Cl}$ onto the dendritic array in an amount thermodynamically dependent on the temperature of the mush. The coldest region of the mush, the channel, promoted heavy solidification, eventually building $\mathrm{NH}_{4} \mathrm{Cl}$ rich segregates adjacent to the channel. The fluid which eventually entered the channel, having been stripped of most of its salt, was a near eutectic, cold but solutally light mixture which rose quickly to feed the plume. This entrainment process continued as the growth front advanced upwards, and the channels were eventually filled with eutectic material leaving behind freckle-type segregates in the resultant cast. During solidification, some channels ceased ejecting fluid, but the remnant of the channels can still be seen. Figure $6.7 \mathrm{~b}$ shows two such channels in the right side of the cast, which have stopped developing and have been overgrown by the liquidus front. 


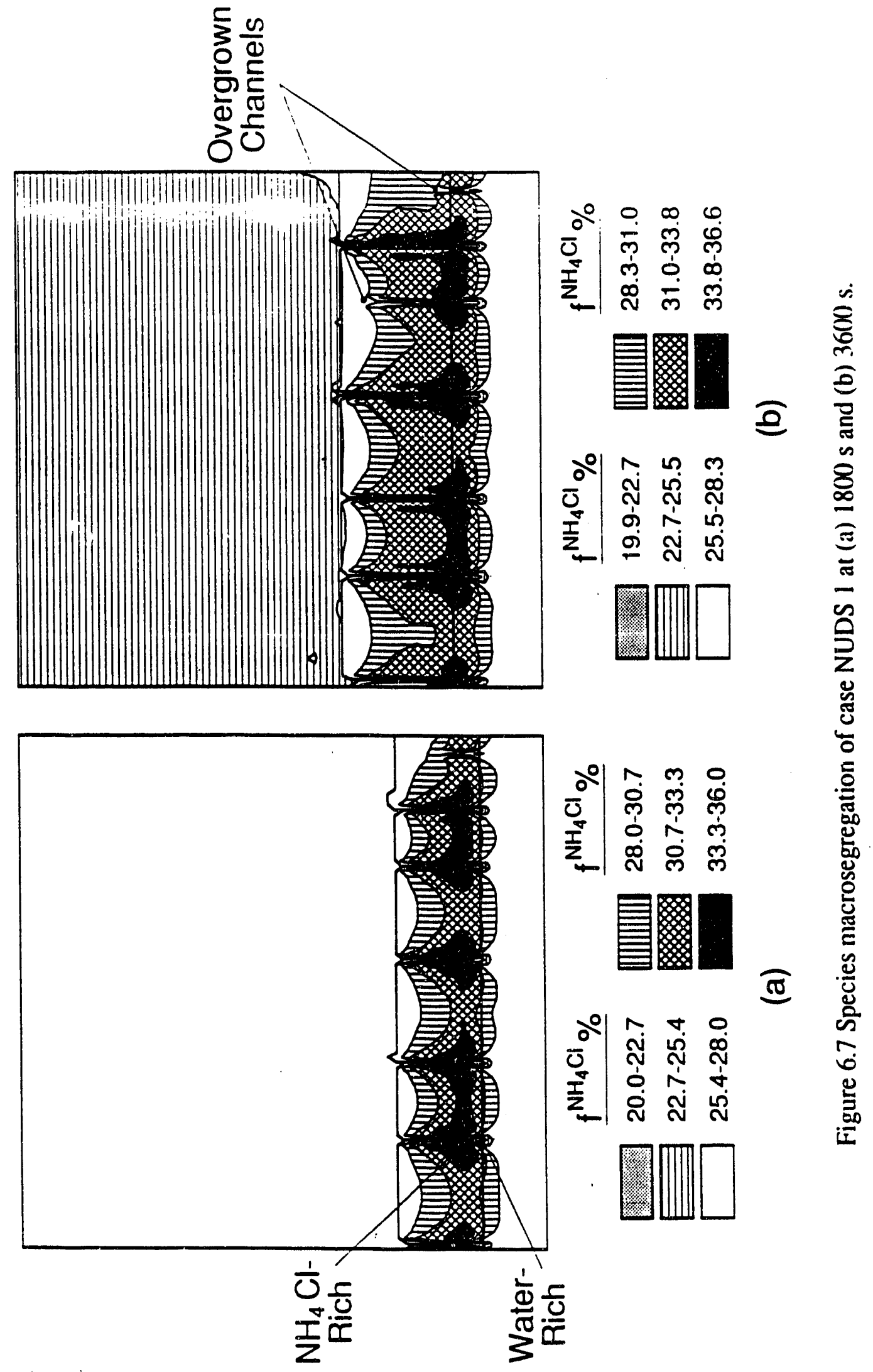


A comparison was made between experimental temperature histories at selected heights from case UDS 1 and the numerical predictions (Fig. 6.8). In general, the numerical simulation under-predicted temperatures in both the mushy region and the melt. The major cause of this discrepancy is, at least in part, due to different boundary conditions at the cold wall. The numerical simulation assumes an immediate and sudden drop in the cold wall temperature to $-30^{\circ} \mathrm{C}$, whereas the cold wall temperature of the actual experiment drops asymptotically to $-30^{\circ} \mathrm{C}$ over the course of the experiment. In fact, the experimental temperatures of the heat exchanger one minute and four minutes after applying coolant were $-20^{\circ} \mathrm{C}$ and $-25^{\circ} \mathrm{C}$, respectively. This small difference in cold wall temperature does not change the development of plumes or the overall macrosegregation patterns in the cast, but it does cause problems when trying to match cooling curves. Other potential sources of discrepancy between the numerical predictions and experimental results include uncertainties in the thermophysical data, as well as the value and nature of the permeability in the mushy region.

Another difference between the predictions and the measurements relates to the vigorous temperature fluctuations in the melt (Fig. 6.8a). While fluctuations in the experiment were generally of large frequency and small amplitude, the model predicted fluctuations at lower frequency and significantly larger amplitude. Predicted temperature fluctuations were associated with buoyant plumes and vortices which rose or fell in the melt (Fig. 6.6b). The large fluctuations are attributed to the model's inability to account for small scale convective phenomena, such as salt fingering and plumes. Again, the grid used to solve the continuum equations was too coarse to provide the resolution needed to consider these effects.

As discussed previously in Chapter 5, the pertinent question arises: is the flow in the melt turbulent? Turbulence does exist in the sense that random, unpredictable fluctuations in temperature and species are prevalent in the cavity. However, these 


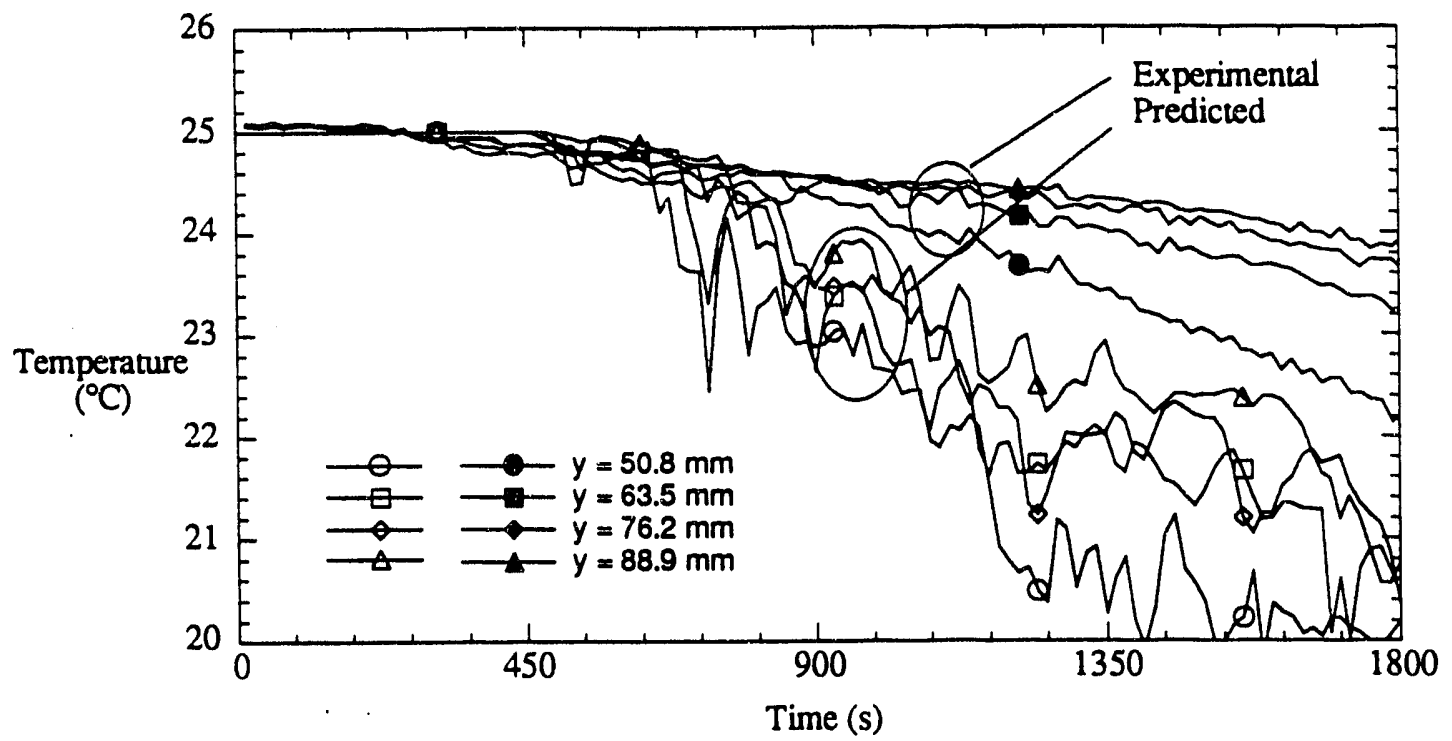

(a)

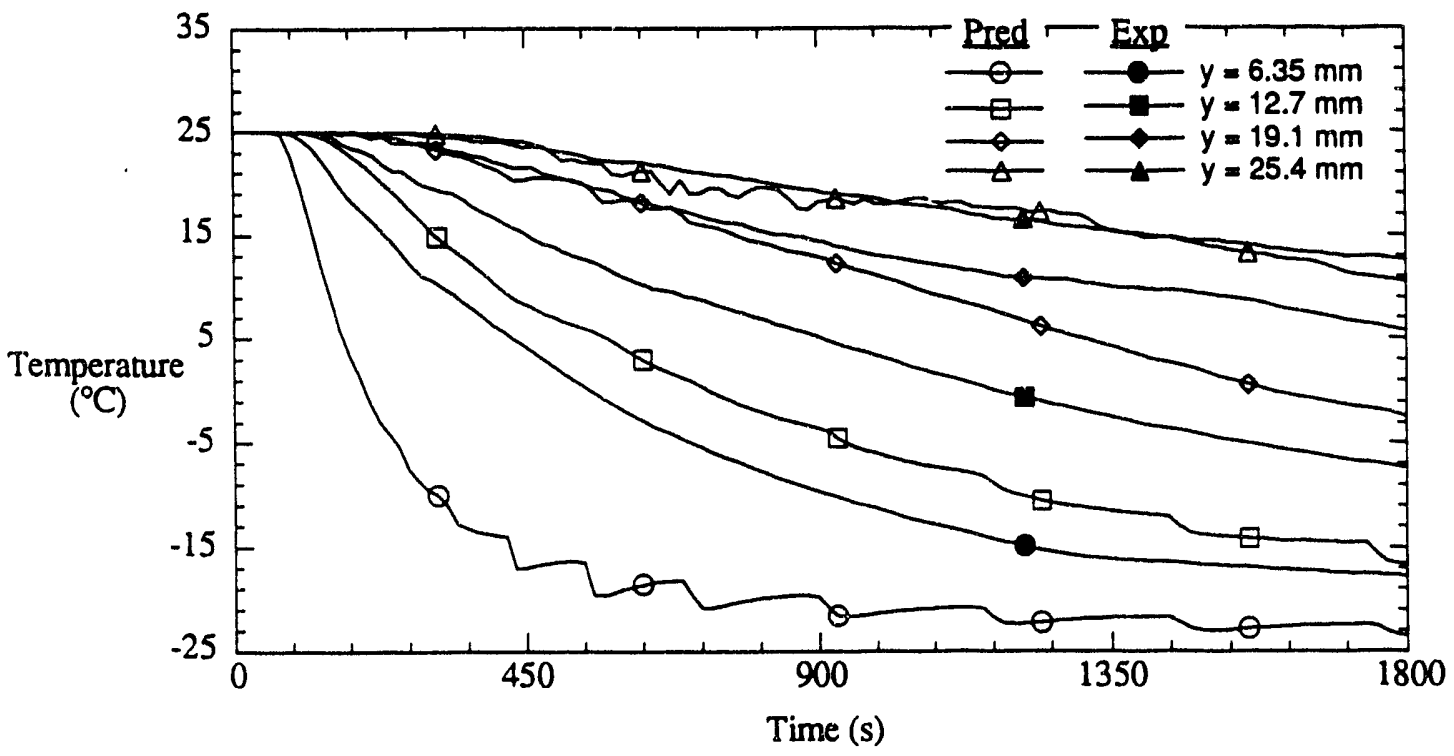

(b)

Figure 6.8 Numerical predictions of temperature histories for case UDS 1 in (a) the melt and (b) the mushy region. 
fluctuations are generated by small scale eddies induced in the flow by double-diffusive salt fingering rather than by shearing. The model could potentially account for this weak turbulence either through refining the grid to resolve the small scale salt fingering or by incorporating a turbulence model to predict double-diffusive transport on a macroscopic scale.

\subsection{Unidirectional Solidification During Rocking}

The continuum equations describing the solidification of a binary alloy undergoing an oscillatory rocking motion were presented in Chapter 3. In developing the equations, some consideration was given to neglecting the inertial terms owing to the relatively slow rocking rate. The strengths of the source terms in the momentum equations were therefore calculated at rocking angles of $45^{\circ}$ and $0^{\circ}$ (vertical) to determine their relative influence. In general, the $y$-momentum equation was dominated by buoyant forces which were an order of magnitude greater than the inertial terms. Coriolis and centripetal accelerations, which were functions of angular velocity, $\Omega$, were zero when the test section was at $45^{\circ}$ from vertical, while angular acceleration $(\partial \Omega / \partial t)$ became zero at the vertical position. On the other hand, the $\mathrm{x}$-momentum equation was found to be strongly influenced by the inertial terms. Although buoyant terms dominate the $\mathrm{x}$-momentum equation when the test section is rocked to $45^{\circ}$, as the test section moves to the vertical position, the $\mathrm{x}$-component of gravity goes to zero and the inertial terms drive fluid motion. Although angular acceleration is zero when $\theta=0^{\circ}$, Coriolis acceleration dominated fluid motion in the melt. Therefore, all inertial acceleration terms were kept in the momentum equations for the rocking computation. In addition, some preliminary calculations of the rocking case with and without the inertial terms showed that the inertial terms were indeed important and contributed to fluid motion. 
The results from the rocking simulation NUDS 4R are presented in Figures 6.9 6.13. Because the simulation was started at the far right position of $\theta=-45^{\circ}$, the test section would return to $\theta=-45^{\circ}$, at 60 second intervals for the rest of the simulation. Therefore, Figures $6.9-6.13$ all represent a time when the test section was tilted to the far right.

Conditions at five minutes were characterized by large, ordered thermally and solutally driven cells which developed in the melt (Fig. 6.9b). The growing mushy region produced a strong thermal boundary layer just above the liquidus interface, and as the fluid turned away from the interface in the lower corner of the cavity, it rose away from the side wall forming a large counter-rotating pocket of fluid. The driving mechanism behind this counter-rotating cell is not entirely understood. Similar to the fluid motions associated with salt fingers seen in experiment UDS 1R (Fig. 5.1c), it was originally speculated that the thermal boundary layer turned away from the side wall because of solutal buoyancy it gained by sweeping the liquidus interface. However, short simulations of static UDS tilted at a $45^{\circ}$ angle showed little evidence of a counter-rotating cell. Similarly, short simulations of a rocking box chilled from below showed only slight evidence of the existence of such a a counter-rotating cell. However, it is important to note that the angular momentum imparted on the fluid body at 300 s, by the rocking motion, tended to drive the fluid in a clockwise direction. Therefore, the cell seems to be a consequence of the combined effects of solutal buoyancy and inertial convection. Sweeping of the growth front can be seen in the isotherms at $t=300$ s, as a bubble of cold fluid bulges from the mushy region (Fig. 6.9c). A similar bubble was present in the isocomps but was not visible due to the nature of the ploting package. The remaining isotherms are smooth and free of any perturbations. Similar patterns in the isocomps showed that the sweeping of the liquidus interface effectively suppressed perturbations in the mushy region. 

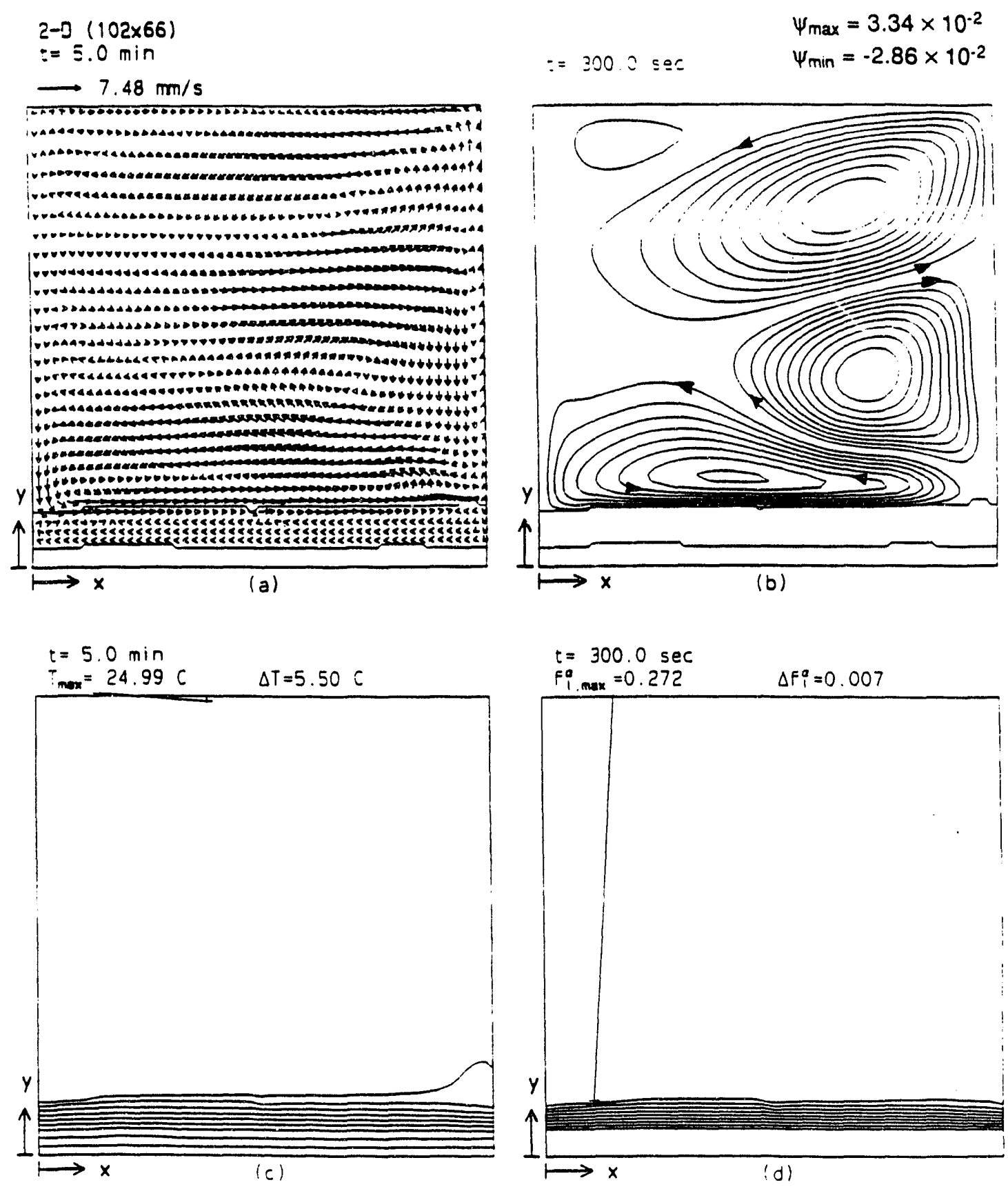

Figure 6.9 Predicted solidification behavior of case NUDS 4R at 300 s: (a) velocity vectors, (b) streamlines, (c) isotherms, and (d) liquid isocomposition. 

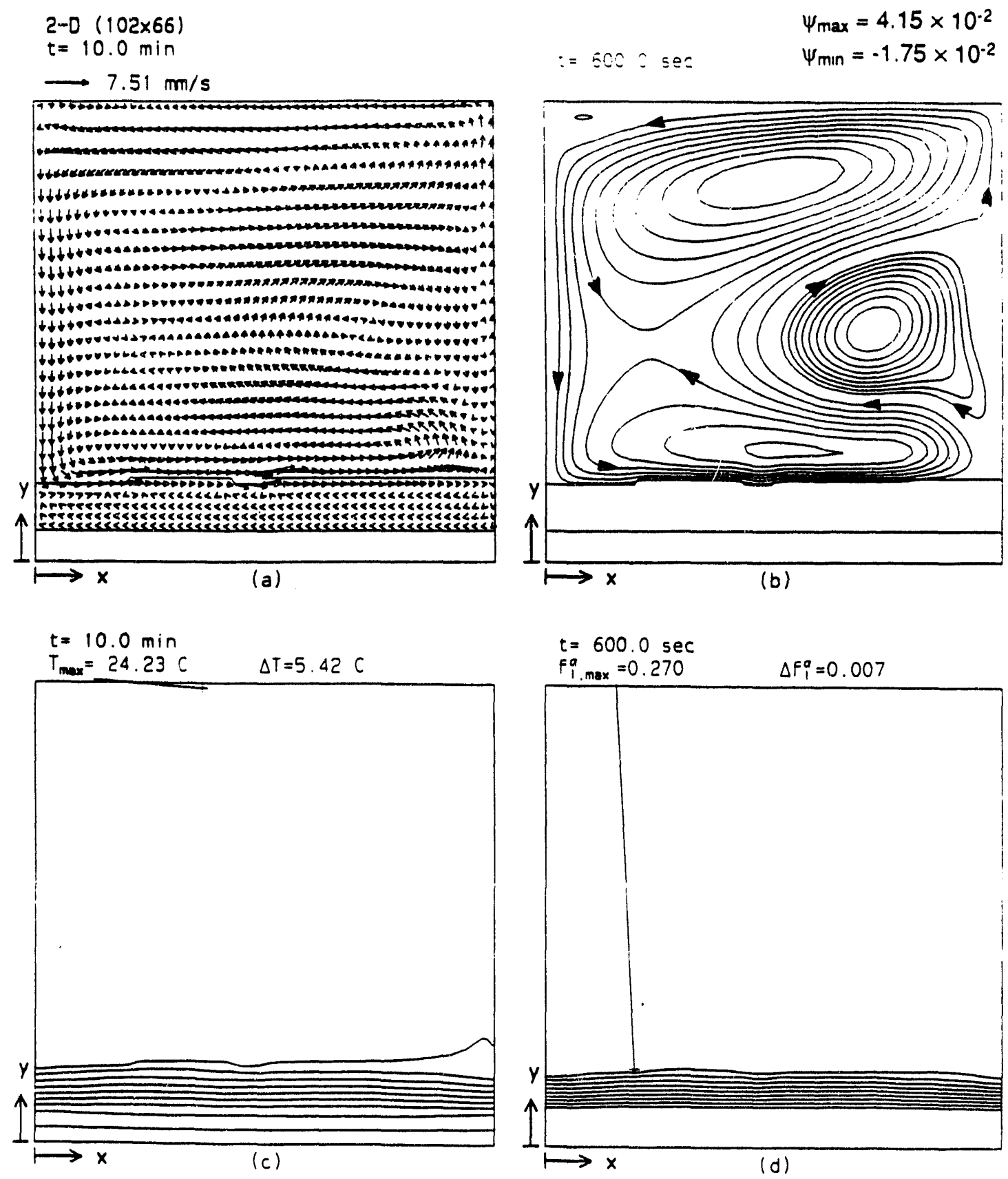

Figure 6.10 Predicted solidification behavior of case NUDS 4R at $600 \mathrm{~s}$ : (a) velocity vectors, (b) streamlines, (c) isotherms, and (d) liquid isocomposition. 

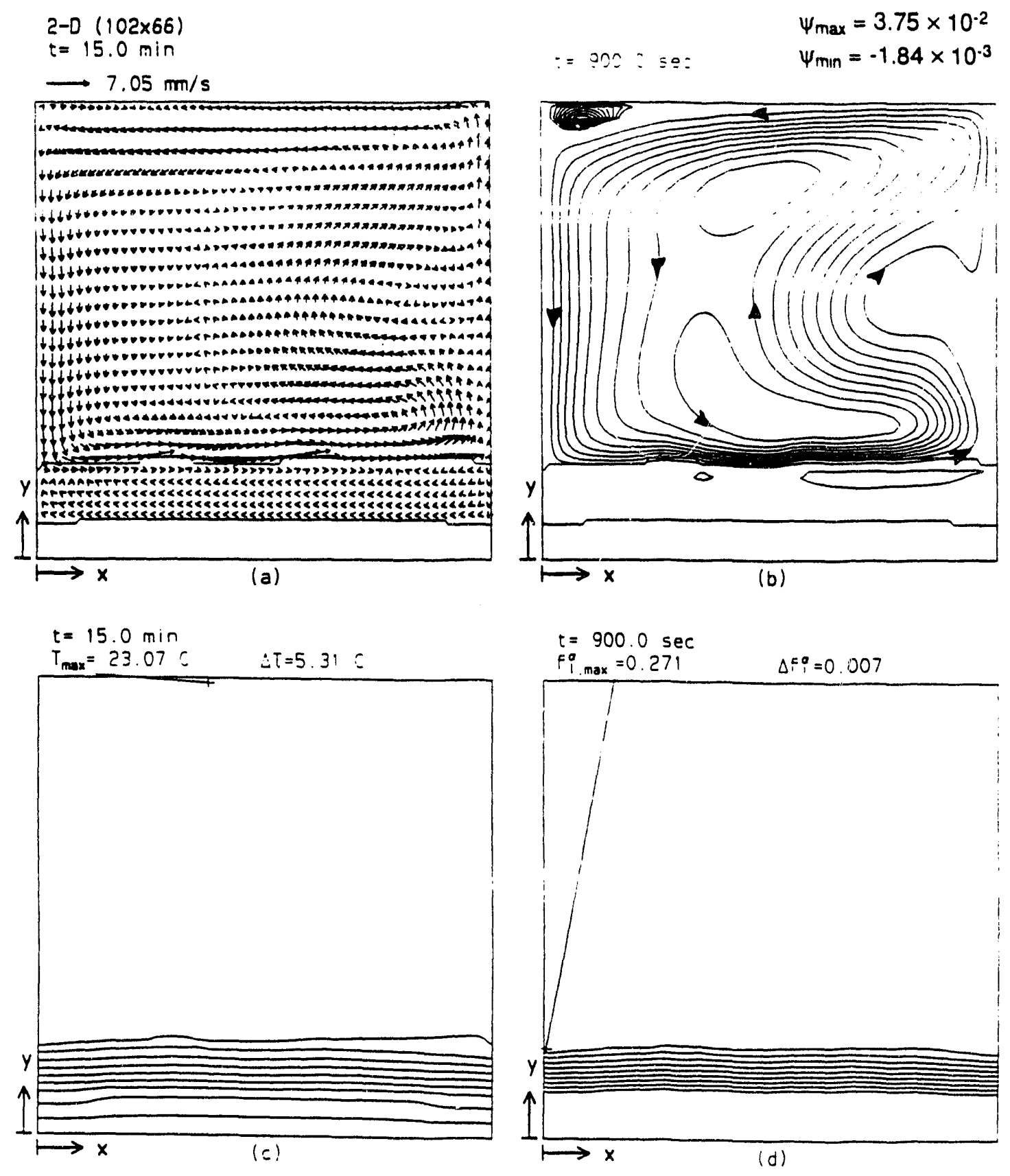

Figure 6.11 Predicted solidification behavior of case NUDS 4R at $900 \mathrm{~s}$ : (a) velocity vectors, (b) streamlines, (c) isotherms, and (d) liquid isocomposition. 
$2-D(102 \times 66)$

$t=30.0 \mathrm{~min}$

$\longrightarrow 7.06 \mathrm{~mm} / \mathrm{s}$

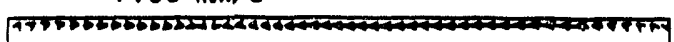

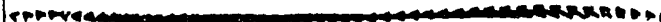
r

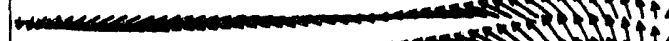

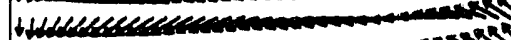

1)

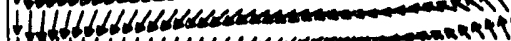

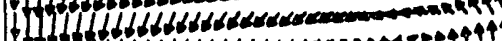

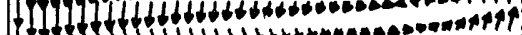

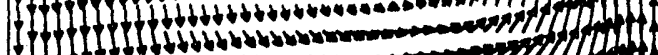

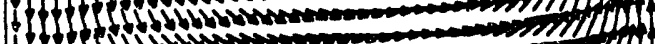
if 1 S 14 H. 1 I 14 S

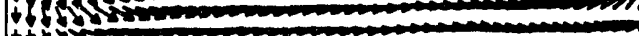
tob

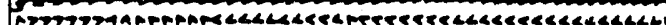

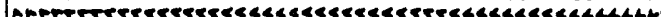

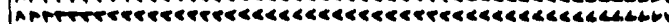

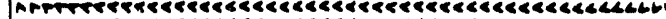

h

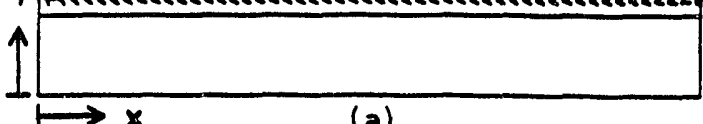

(a)

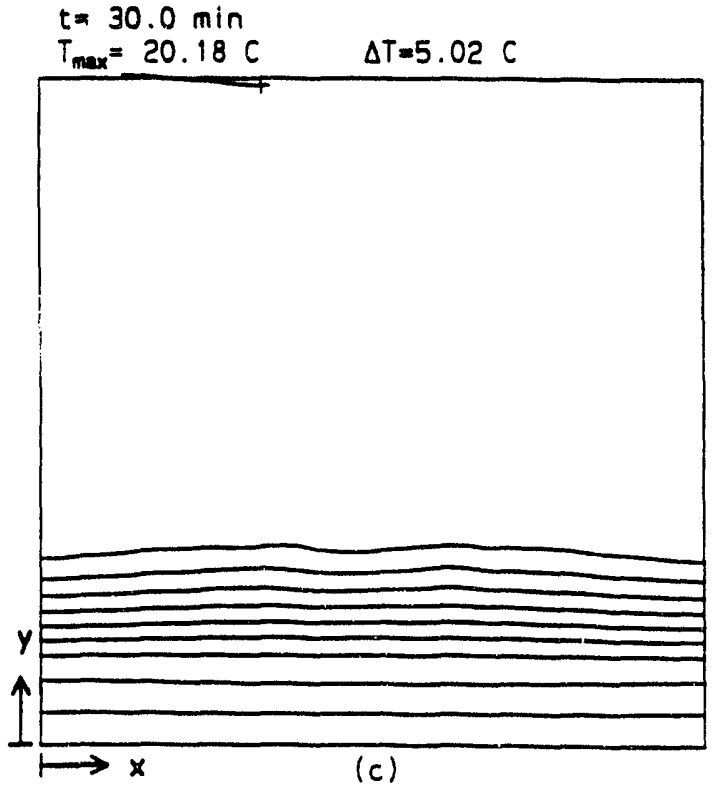

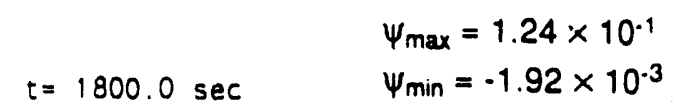
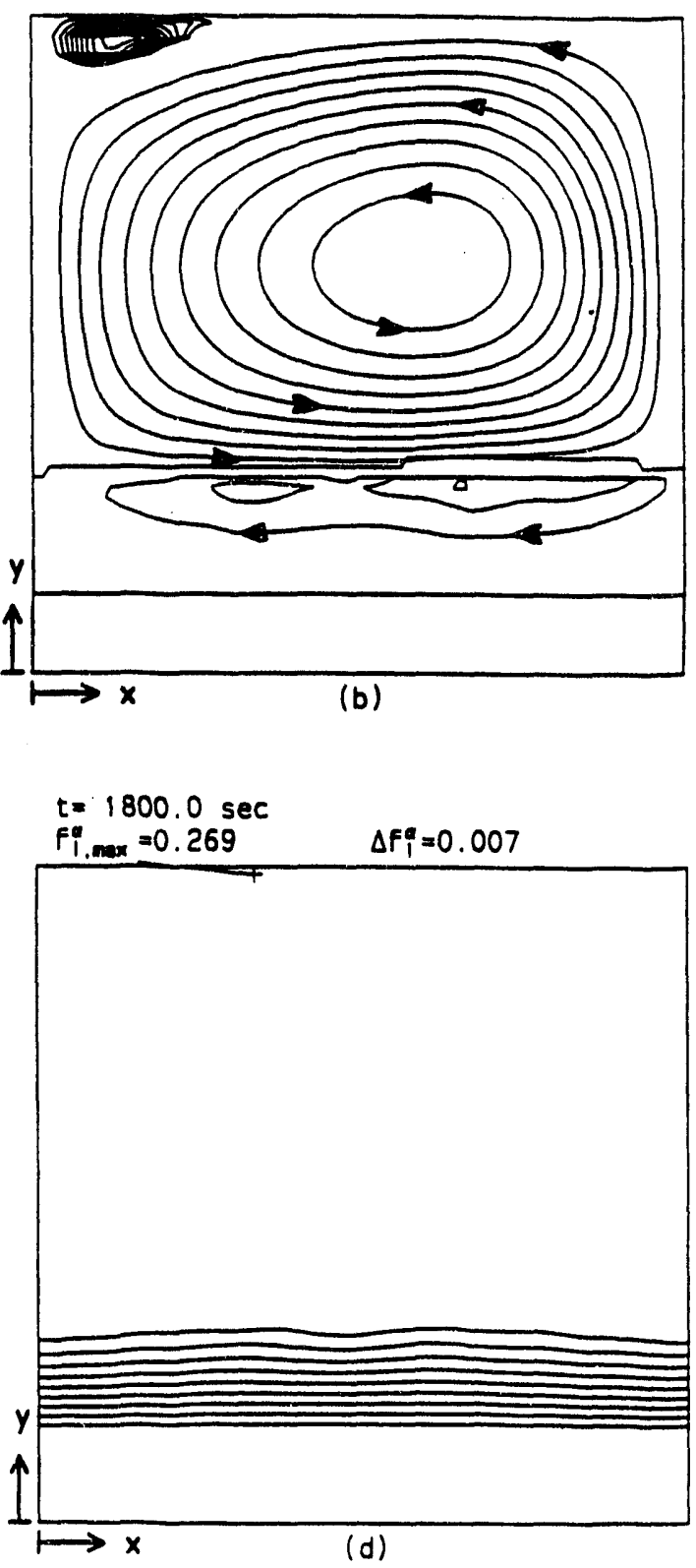

Figure 6.12 Predicted solidification behavior of case NUDS 4R at $1800 \mathrm{~s}$ : (a) velocity vectors, (b) streamlines, (c) isotherms, and (d) liquid isocomposition. 
2-D (102x66)

$t=60.0 \mathrm{~min}$

$\longrightarrow 4.50 \mathrm{~mm} / \mathrm{s}$

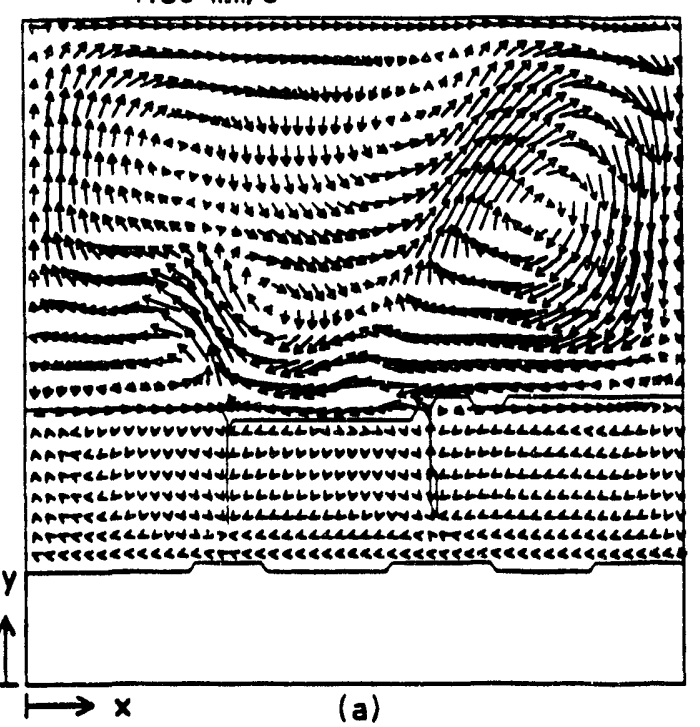

\section{$t=60.0 \mathrm{~min}$}

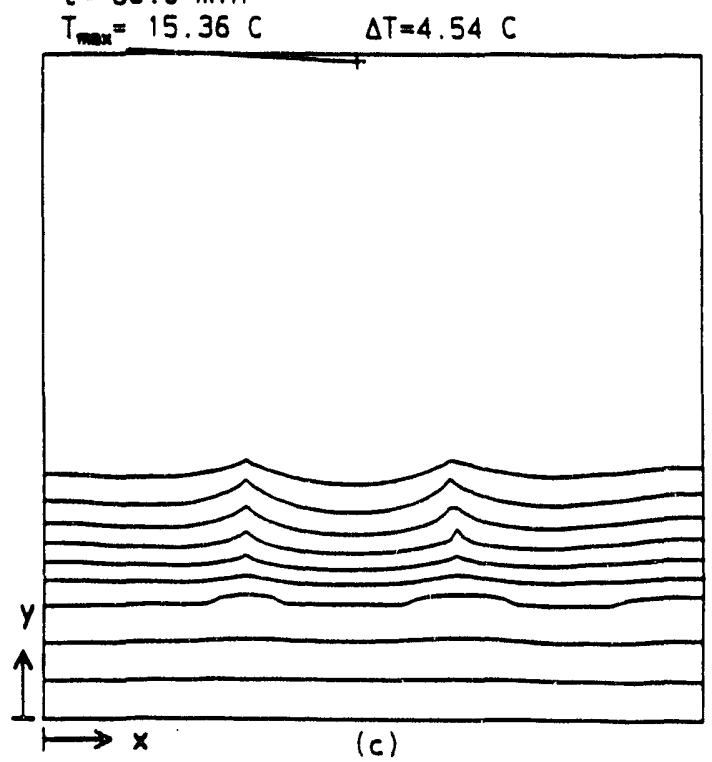

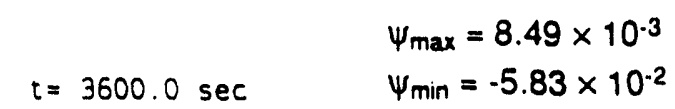
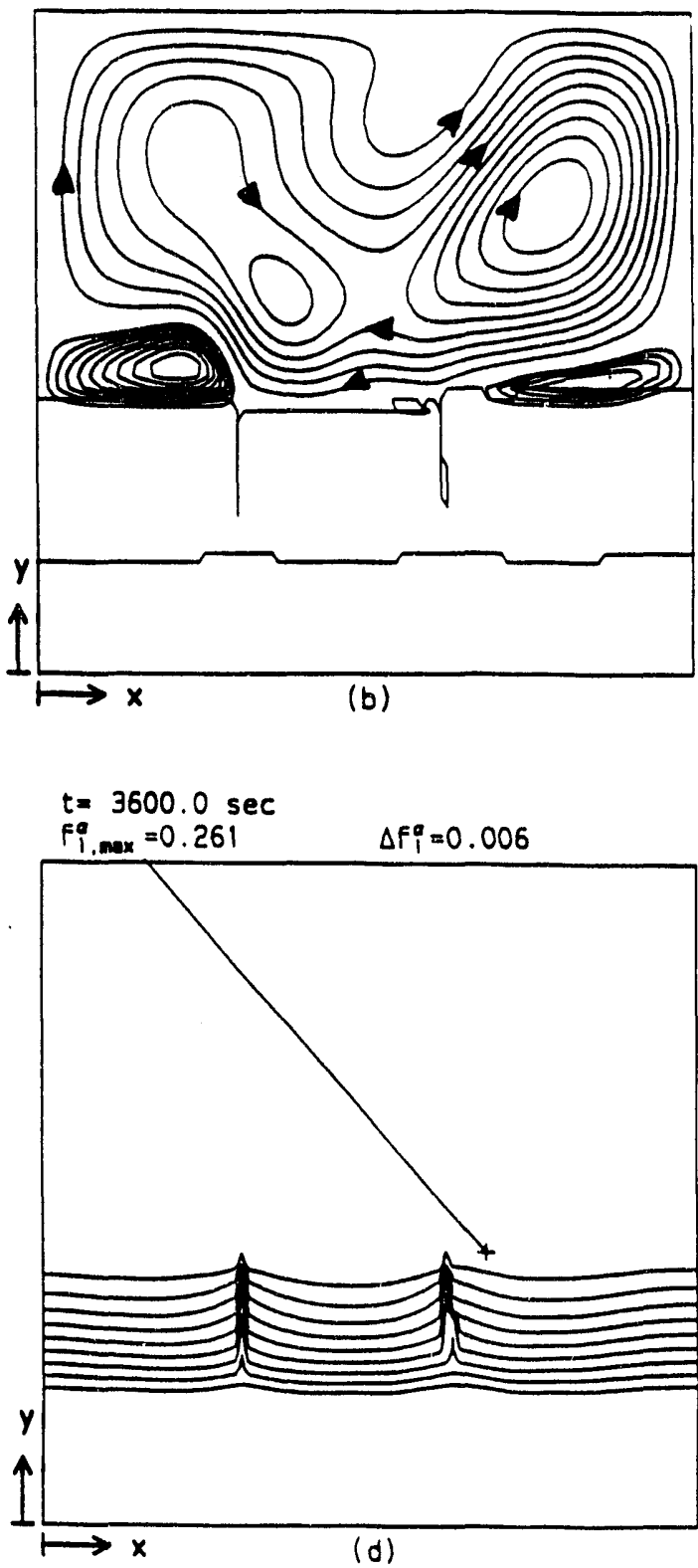

Figure 6.13 Predicted solidification behavior of case NUDS 4R at $3600 \mathrm{~s}$ : (a) velocity vectors, (b) streamlines, (c) isotherms, and (d) liquid isocomposition. 
The liquidus interface was generally planar; however, as thermal boundary layers developed along the growth front, solidification was enhanced at downwind regions of the boundary layer owing to fluid chilling. To satisfy continuity, warm fluid from the melt would impinge on the mushy region at the top of the thermal boundary layer, remelting the liquidus interface. As the test section rocked in the other direction, the process would reverse, with crystal growth favored on the new downwind region of the boundary layer and remelting on the upper region. Flow within the mushy region, driven by solutal buoyancy, traversed across the porous zone eventually exiting near the left end of the region (Fig. 6.9a).

Conditions at ten minutes were little changed from those at five minutes (Fig. 6.10). Flow in the melt was still dominated by thermal buoyancy, while solutally buoyant fluid flowed upward (in the $\mathrm{x}$ direction) through the mushy region. The counter-rotating vortex in the lower right comer of the cavity was still present, and a cold pocket of fluid still bulged above the liquidus interface. Also, mushy region isotherms and isocomps were still flat at ten minutes, and hence free of density perturbations (Fig. 6.10c and d).

At 15 minutes into the simulation, strong thermally driven flow along the growth front continued to keep the temperature and composition fields smooth (Fig. $6.11 \mathrm{c}$ and d). However, because of the decreased rate of heat removal, less water-rich fiuid was rejected by the mushy region and convection patterns in the melt weakened siightly. In addition, the counter-rotating vortex in the lower corner of the cavity weakened considerably. This weakening seemed to be a manifestation of two events. First, the decrease in the supply of water-rich fluid to the melt acted to reduce the influence of solutal buoyancy on the vortex. Secondly, the decrease in the distance between the mushy region and upper wall tended to damp counter-rotating fluid motion high in the melt, promoting instead, the dominant thermally driven cell. The thickening mushy region established a wider path for water-rich fluid to progress upward through the crystalline structure, and the liquidus interface 
remained comparatively flat, which is in contrast to the convex growth front observed in the experiments.

The inability of the model to predict the convex growth front is puzzling. Indeed, predicted isotherms and isocomps show a convex profile resulting from impingement of warm, salt-rich fluid during the rocking cycle, but the predicted liquidus interface remained relatively flat. One possible explanation points to the coarseness of the grid, which may render the model incapable of determining the relatively small slope of the convex front. Yet another possibility may be the model's assumption of thermodynamic equilibrium at the liquidus interface. In its present form, the model attributes a mushy region to any control volume where the temperature drops below the liquidus temperature, according to thermodynamic equilibrium. However, in actuality, sizeable undercooling existed in the vicinity of the growth front. This undercooling, which the model completely neglects, may have augmented the severity of the convex shape.

Only one major thermally driven cell remained in the melt after 30 minutes (Fig. $6.12 \mathrm{~b})$. The melt was nearly isothermal, and very little energy or species transport occurred across the liquidus interface. The mushy region had thickened considerably, and solutally light fluid continued to rise within the structure (Fig. 6.12a). Although temperature and composition fields were largely free of perturbations, small perturbations in the velocity vectors at the liquidus interface hinted at the channeling which would later develop at approximately 2500 seconds.

The two channels that formed reached mature states of development one hour into the simulation (Fig. 6.13). The concomitant plumes vigorously rejected light, water-rich fluid into the melt which drove a large solutal convection cell (Fig. 6.13b). Cintrary to the thermally driven cells found earlier in the simulation, the solutally driven cell rotated clockwise, advecting fluid from the channel into the melt. Two smaller thermally driven cells were located just above the growth front on the outside of the channels. As evidenced 
by the pocket of fluid which formed at the base of the channel on the right and the steep spikes which developed in the isocomps and isotherms, the channels were well developed and appeared stronger than channels simulated under static conditions. Because just two channels are relieving interdendritic buoyancy potential similar to that released by five channels under static conditions, this development was not surprising. With the strong channel development, macrosegregation was equally severe.

Before channels had developed, macrosegregation at 1800 seconds was markedly benign (Fig. $6.14 \mathrm{a}$ ). With the $\mathrm{NH}_{4} \mathrm{Cl}$ composition varying by only $\pm 3 \%$ from the nominal composition, salt aggregated in the lower outside regions of the cast and water-rich areas formed near the center. The salt-rich areas formed early in the simulation, as solution entrained from the melt deposited $\mathrm{NH}_{4} \mathrm{Cl}$ onto the crystal structure. The remaining waterrich fluid advected to the center of the cast, forming localized salt-deficient regions. Although present, the segregates were not severe and were a distinct improvement over freckle-type segregates formed under static conditions.

The macrosegregation pattern at one hour was much different (Fig. 6.14b). As anticipated, the channels which did form were well developed, and the channel strength resulted in two severe freckle-type segregates. Morphologically similar to segregates formed under static conditions, the freckles consisted of a salt-rich segregate surrounding the channel itself. Maximum composition values adjacent to the channels were approximately $35 \%$ wt. $\mathrm{NH}_{4} \mathrm{Cl}$, while near eutectic fluid flowed within the channel. However, because they developed later in the process, the freckles which formed with rocking were positioned much higher in the melt than their statically grown cousins. Had the simulation been allowed to completely solidify, two freckle segregates would develop in the cast. They would extend vertically from the location of the channels shown in Figure $6.14 \mathrm{~b}$ to the upper regions of the cast, and banded segregation would develop in the remaining areas of the cast with decreasing $\mathrm{NH}_{4} \mathrm{Cl}$ concentrations toward the top. 


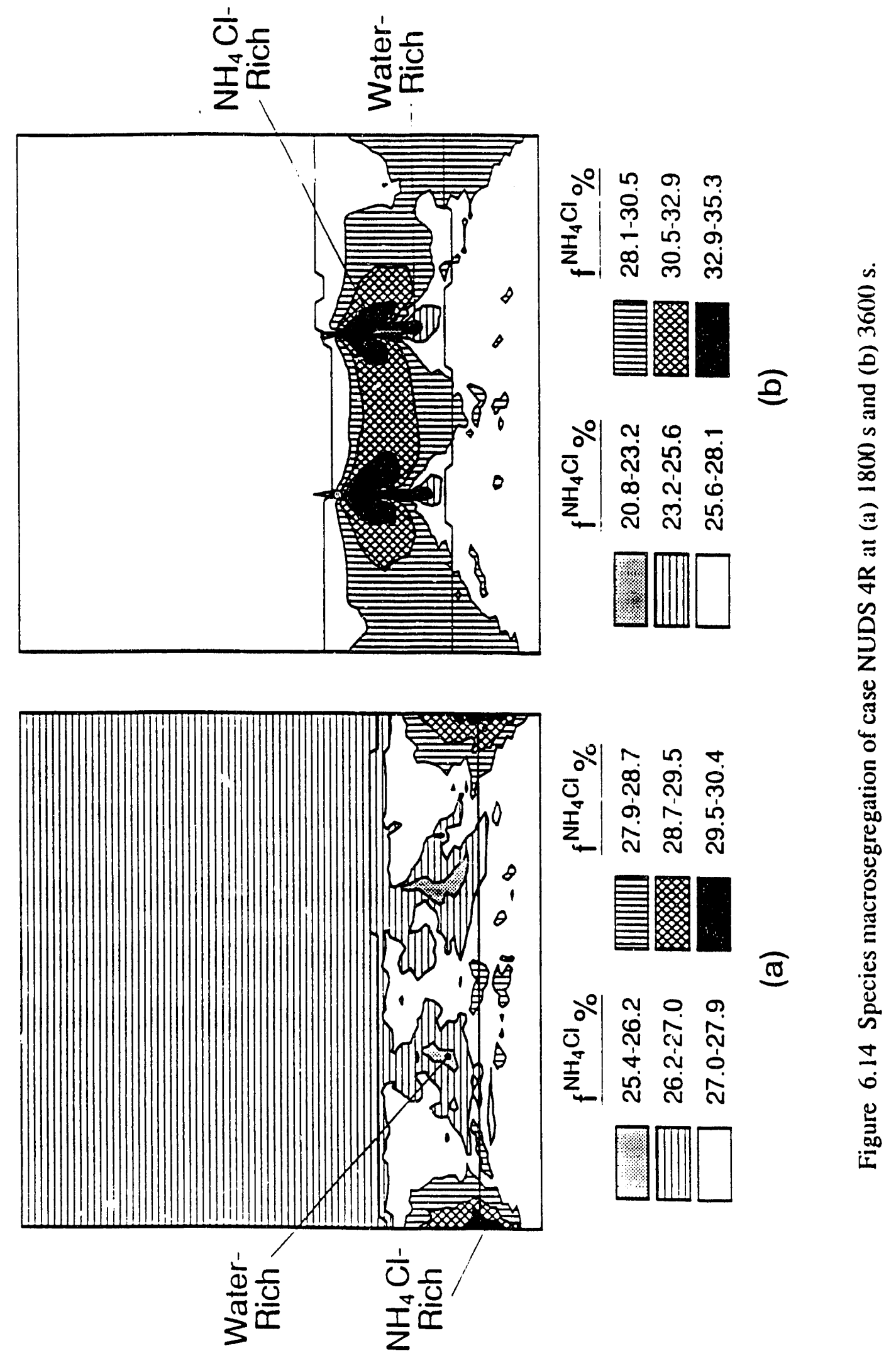


In order to quantify the difference between macrosegregation in the rocking and static simulations, an effective global standard deviation of species was calculated for each simulation. Defined as

$$
M_{\mathrm{rms}}=\left[\frac{1}{\mathrm{~V}} \int_{\mathrm{V}}\left(\mathrm{f}^{\alpha}-\mathrm{f}_{\mathrm{o}}^{\alpha}\right)^{2} \mathrm{dV}\right]^{1 / 2}
$$

the root-mean-square of macrosegregation gives an indication of the severity of species separation over the course of an experiment. As suspected, rocking greatly improved segregation in the cast (Fig. 6.15). For the static simulation, segregation was relatively small for that period of time before convection began. However, once plumes developed, large amounts of water-rich fluid were rejected into the melt and macrosegregation increased rapidly. On the other hand, with rocking, suppression of channels early in the simulation resulted in only a small increase in macrosegregation. However, once again, as channels developed in the rocking simulation, segregation rose steeply as species separated.

At this point, it would be useful to drop back in time and elucidate key features of an oscillation period from $t=600$ to 630 s. Figure 6.10 shows the conditions in the cast at 600 s into the experiment when the test section is positioned at $\theta=-45^{\circ}$ (far right) and has zero angular velocity. As mentioned before, a strong thermal boundary layer descended along the liquidus interface, driving a primary circulation cell in the melt and sweeping temperature and composition perturbations from the liquidus interface. However, owing to the rocking motion, a small counter-rotating cell existed in the lower right corner of the melt.

As the test section rocked back toward vertical, inertial effects contributed to growth of the counter-rotating cell, such that at $605 \mathrm{~s}$, with a rocking position of $-38.4^{\circ}$, a large clockwise rotation cell occupied the center of the cavity (Fig. 6.16). The cell resulting from 
Macrosegregation RMS

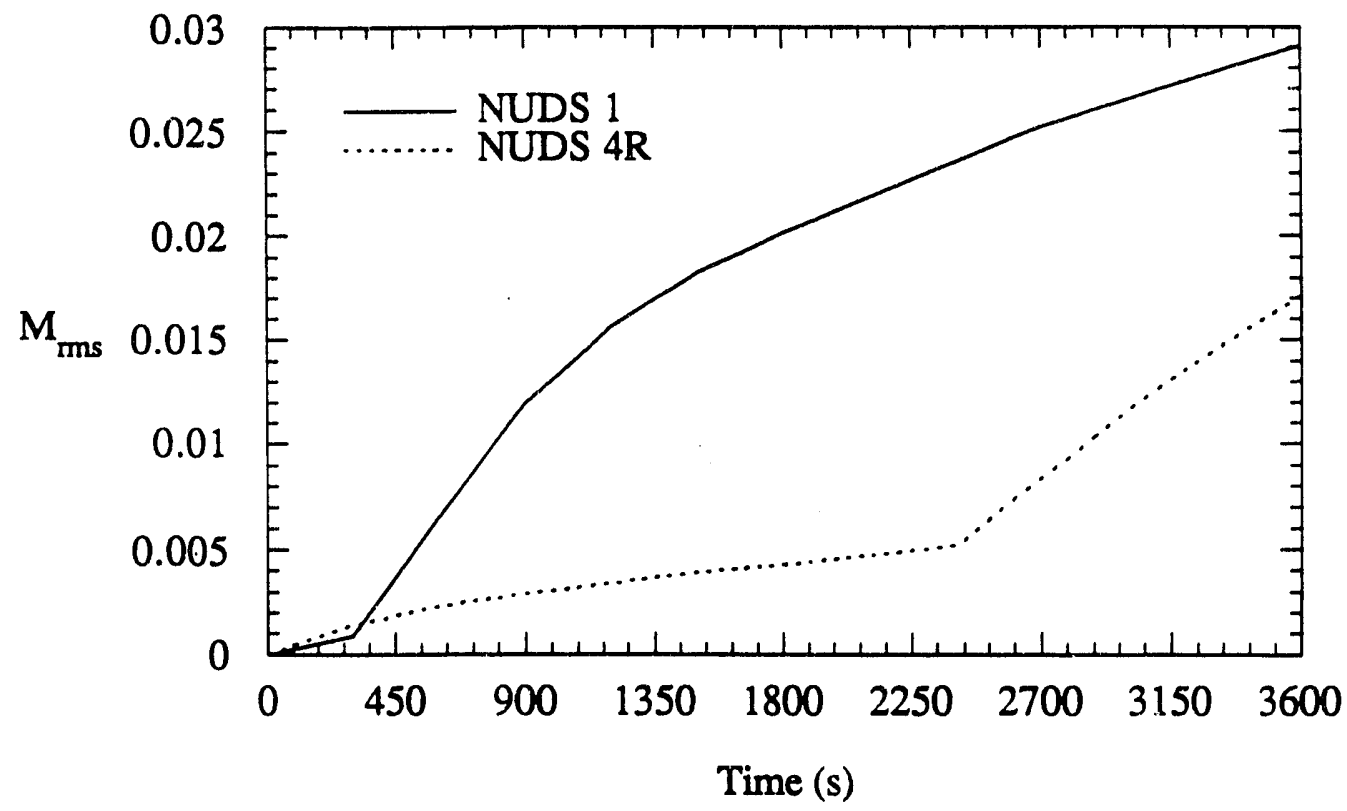

Figure 6.15 Comparison of overall macrosegregation for static solidification and solidification with rocking. 
$2-0(102 \times 66)$

$t=10.1 \mathrm{~min}$

\section{$\longrightarrow 5.05 \mathrm{~mm} / \mathrm{s}$}

Trom

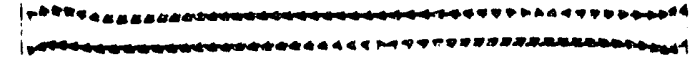
| |t+

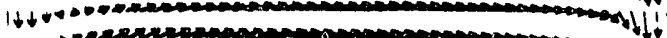
$\mid+t+\circ 0$ o

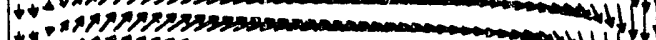

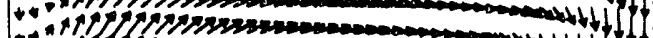

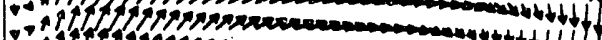

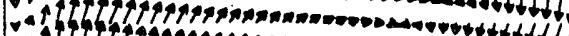
vat - 4 I T

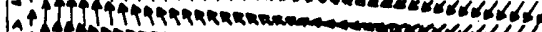

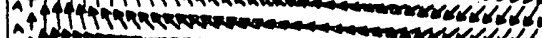
the - the

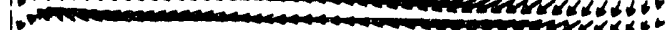
14 os

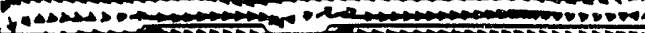
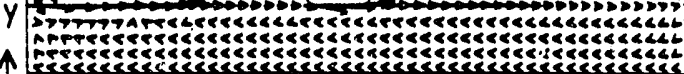

1

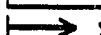

(a)

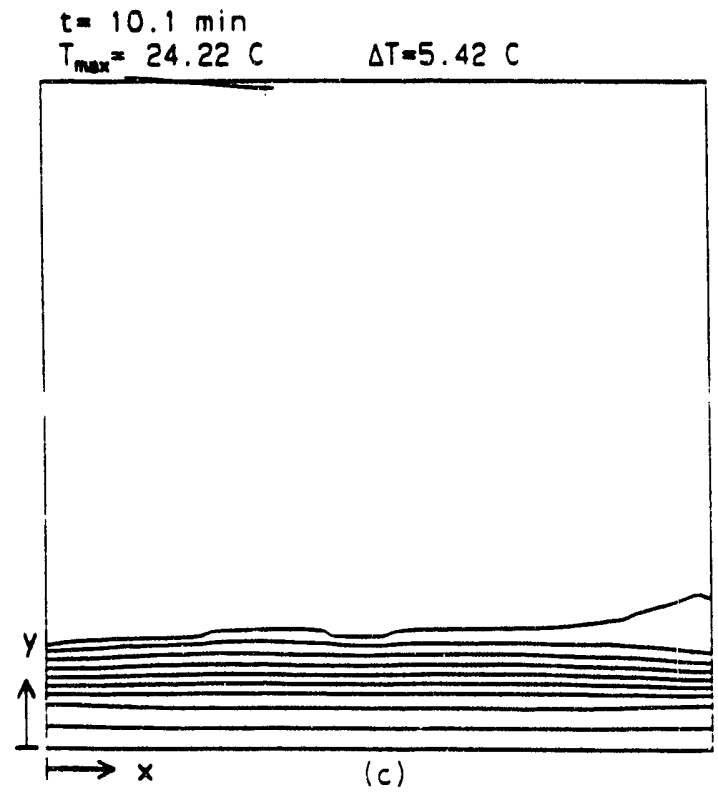

$$
\begin{array}{ll}
t=605.0 \mathrm{sec} & \Psi_{\max }=9.83 \times 10^{.3} \\
& \Psi_{\min }=-5.95 \times 10^{0.2}
\end{array}
$$
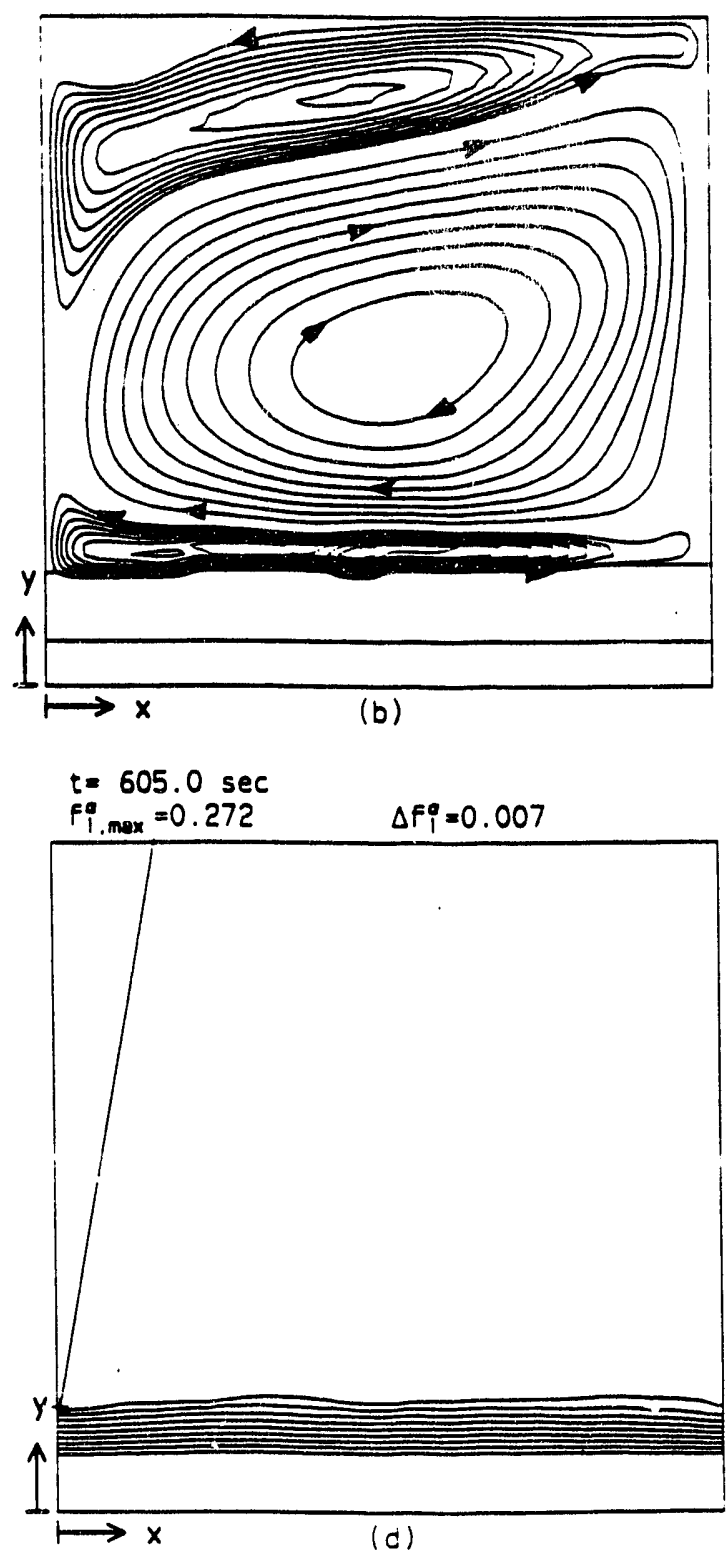

Figure 6.15 Predicted solidification behavior of case NUDS $4 \mathrm{R}$ at $605 \mathrm{~s}\left(\theta=-38.4^{\circ}\right)$ : (a) velocity vectors, (b) streamlines, (c) isotherms, and (d) liquid isocomposition. 
thermal boundary layer development at the growth front was suppressed by this developing inertial cell and was barely perceptible at 610 s, when the test section had rocked to $\theta=$ $-22.5^{\circ}$ (Fig. 6.17). The period of rocking from the maximum tilt to the vertical position was characterized by increasingly robust inertial convection, as the angular velocity of the box approached its maximum. In turn, inertial rotation of the enclosed fluid achieved its maximum strength. Therefore, although thermal convection ceased as the test section rocked to the vertical position $\left(\theta=0^{\circ}\right)$, a strong, negatively rotating cell dominated the melt, continuing to sweep the liquidus interface (Fig. 6.18). Benefitting from this convection, the isotherms and isocomps remained smooth and free of perturbations.

As the test section rocked from the vertical to the left, the angular velocity of the cavity, and hence inertial convection, decreased. However, augmented by the existing convection cell, thermal buoyancy redeveloped, producing strong liquidus washing when the test section was positioned at $\theta=22.5^{\circ}$ (Fig. 6.19). Within the mushy region, waterrich fluid traversed to the right, driven by solutal buoyancy. Rocking further to $\theta=38.4^{\circ}$, convection from both thermal buoyancy and the rocking motion combined to produce the largest velocities seen in the oscillation period, resulting in continued vigorous washing of the growth front (Fig. 6.20). Finally, as the test section came to a halt at the far left position $\left(\theta=45^{\circ}\right)$, the thermal boundary layer above the mushy region drove the primary convection cell in the melt (Fig. 6.21). However, due to the absence of inertial effects, convection in the melt was substantially reduced relative to conditions at $t=625$ or $620 \mathrm{~s}$. Now a counter-rotating cell developed in the lower left corner of the cavity. The cell is an artifact of the angular momentum transferred to the fluid by the enclosure during the rocking sweep.

Predicted cooling curves from simulation NUDS $4 \mathrm{R}$ and measures cooling curves from experiment UDS 4R are contrasted in Figure 6.22. Two salient differences between predictions and experimental results are revealed: 1) predicted temperature stratifications in 


\section{2-D $(102 \times 66)$ \\ $t=10.2 \mathrm{~min}$}
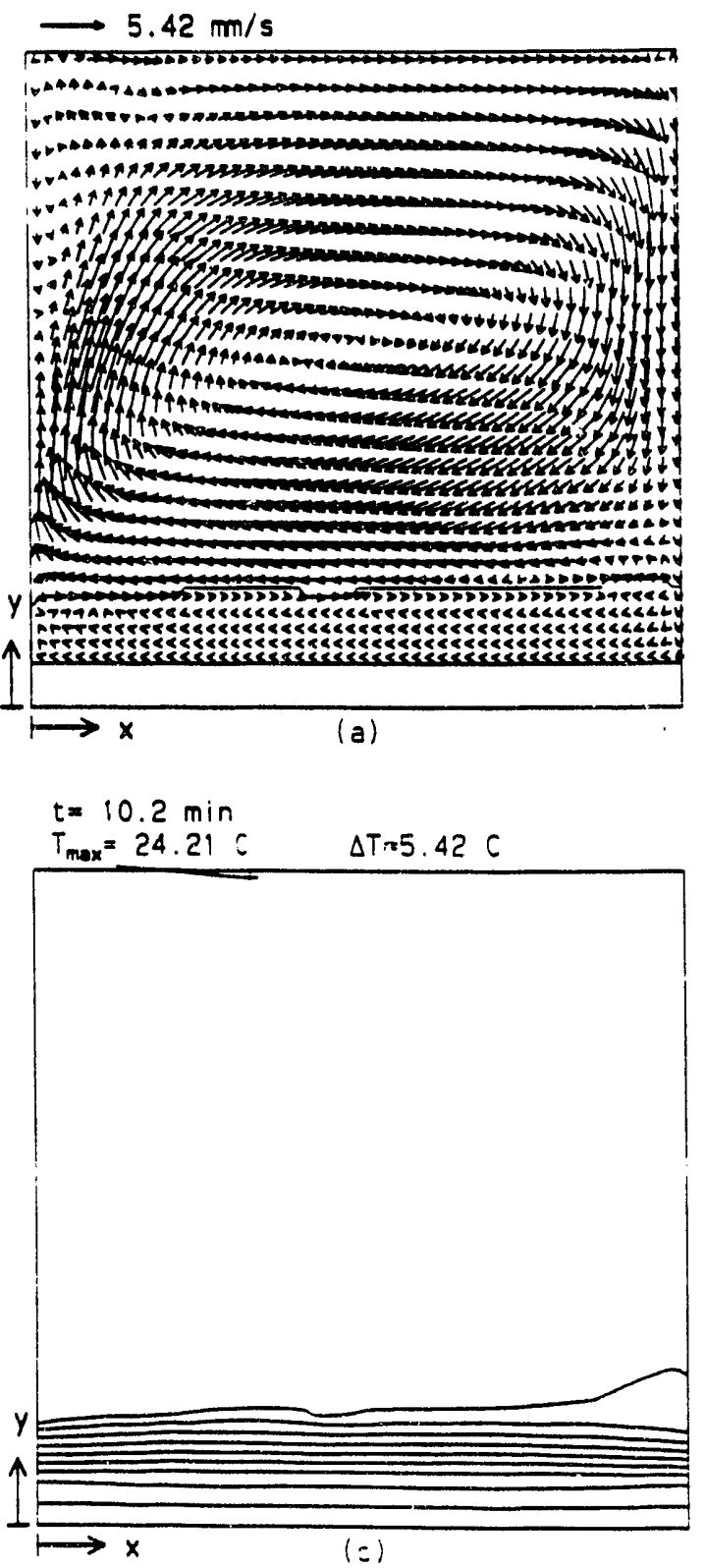

$r=610.0 \mathrm{sec}$

$\psi_{\max }=2.24 \times 10^{.3}$

$\psi_{\text {min }}=-9.08 \times 10^{-2}$

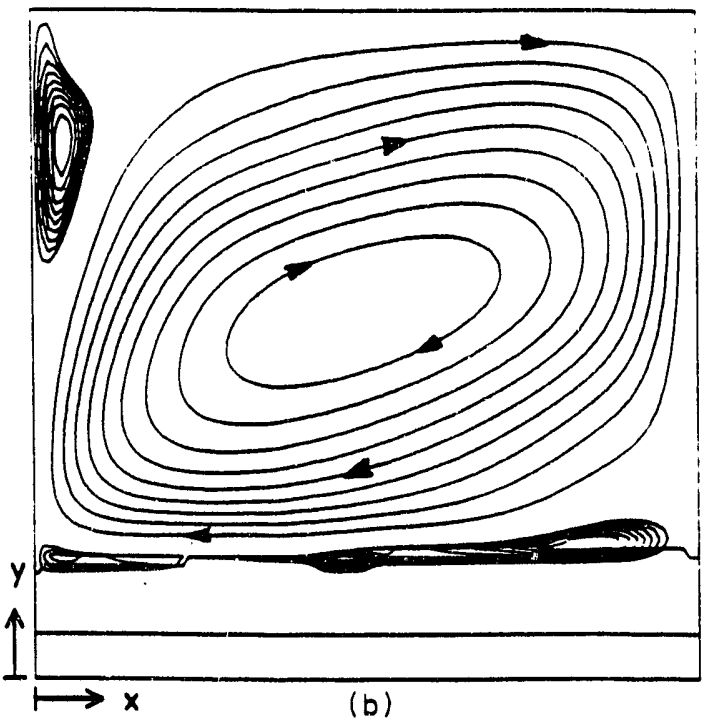

$t=610.0 \mathrm{sec}$

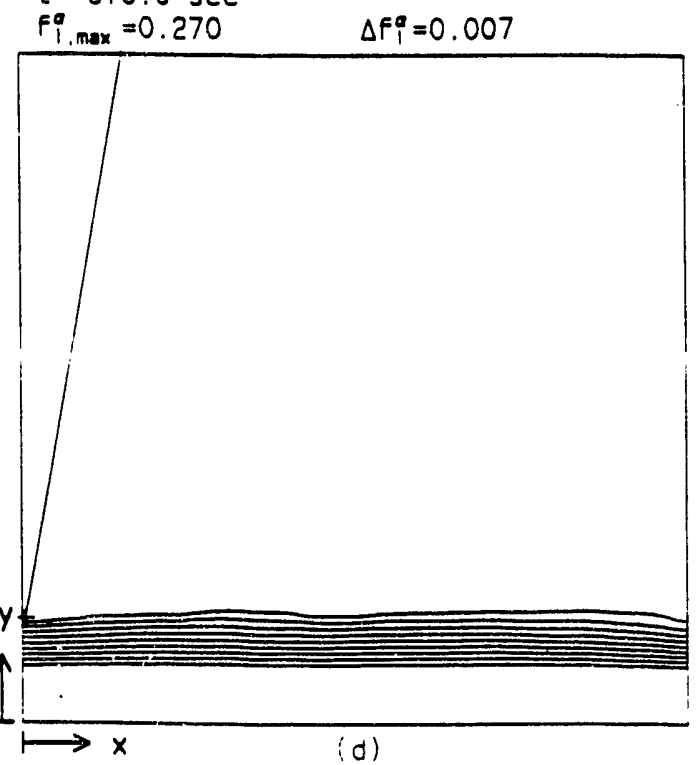

Figure 6.17 Predicted solidification behavior of case NUDS $4 \mathrm{R}$ at $610 \mathrm{~s}\left(\theta=-22.5^{\circ}\right)$ : (a) velocity vectors, (b) streamlines, (c) isotherms, and (d) liquid isocomposition. 
2-D (102x66)

$t=10.3 \mathrm{~min}$

$\longrightarrow 7.60 \mathrm{~mm} / \mathrm{s}$

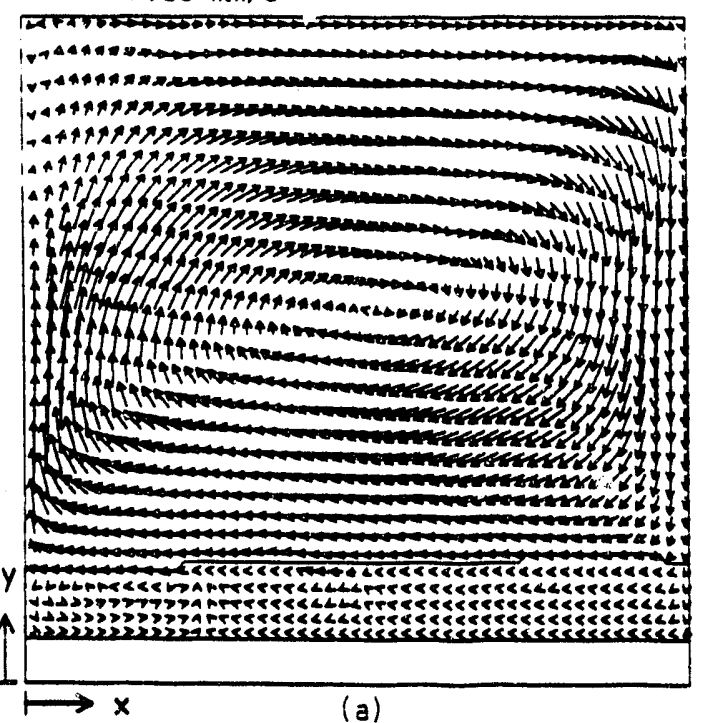

$t=10.3 \mathrm{~min}$

$T_{\max }=24.18 \mathrm{C}$

$\Delta T=5.42 C$

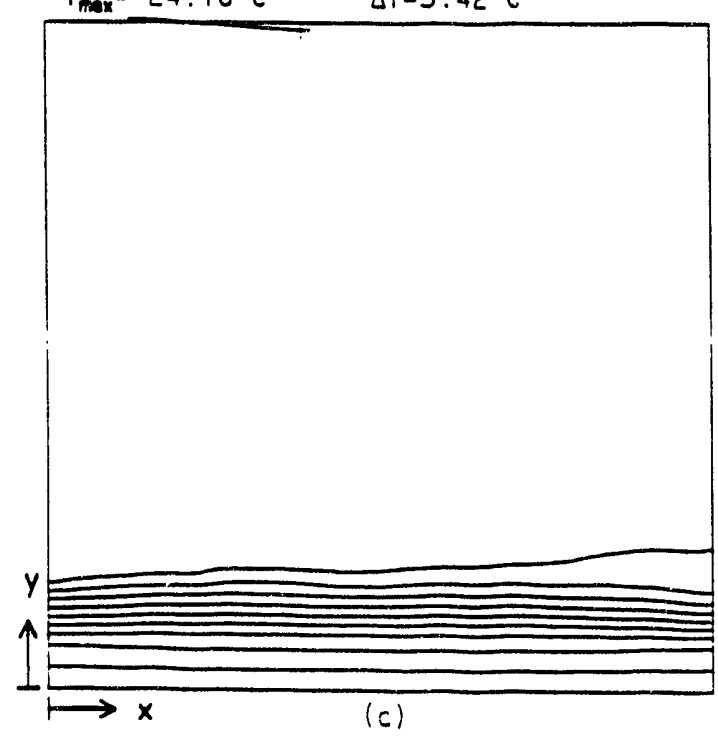

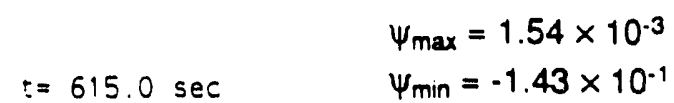

(
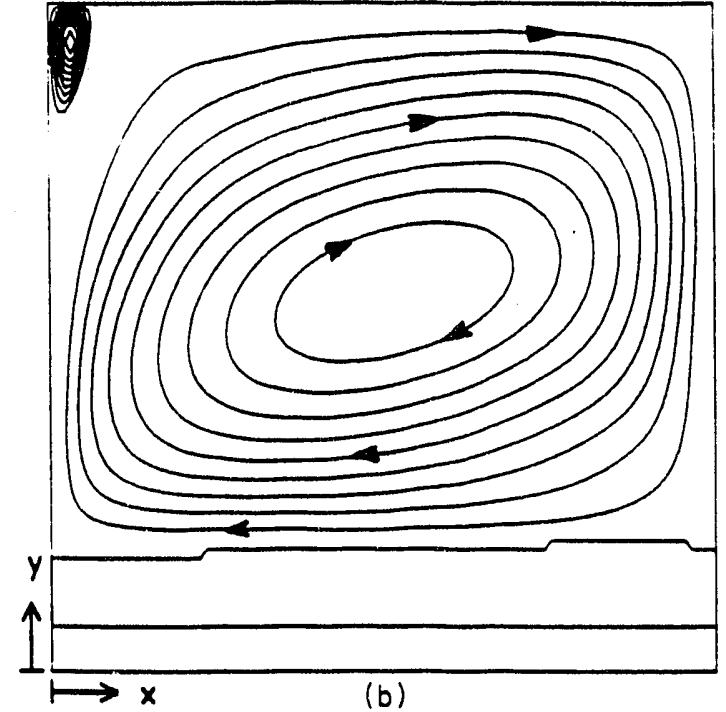

$t=615.0 \mathrm{sec}$

$f_{i \text { max }}=0.270$

$\Delta F_{i}=0.007$

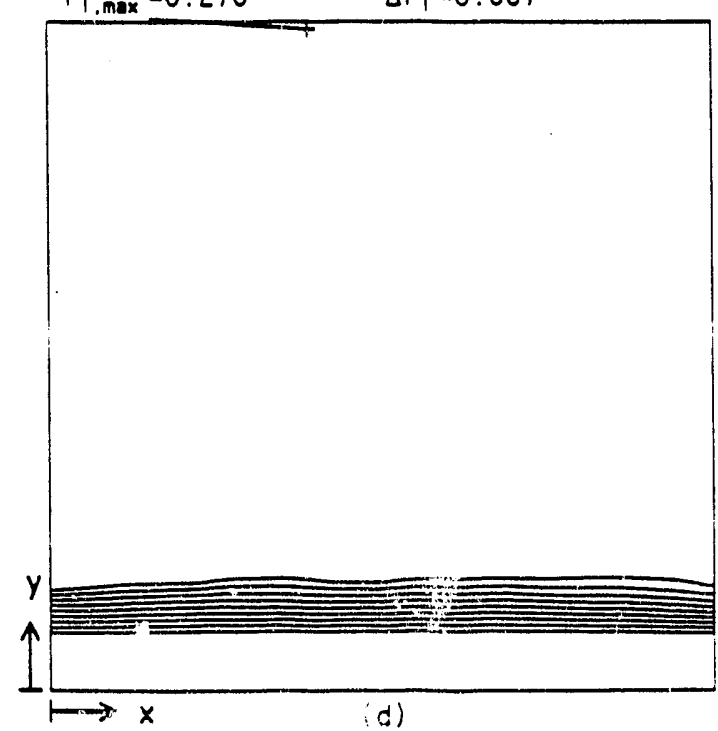

Figure 6.18 Predicted solidification behavior of case NUCS at $615 \mathrm{~s}\left(\theta=0^{\circ}\right)$ : (a) velocity vectors, (b) streamlines, (c) isotherms, and (d) liquid isocomposition. 

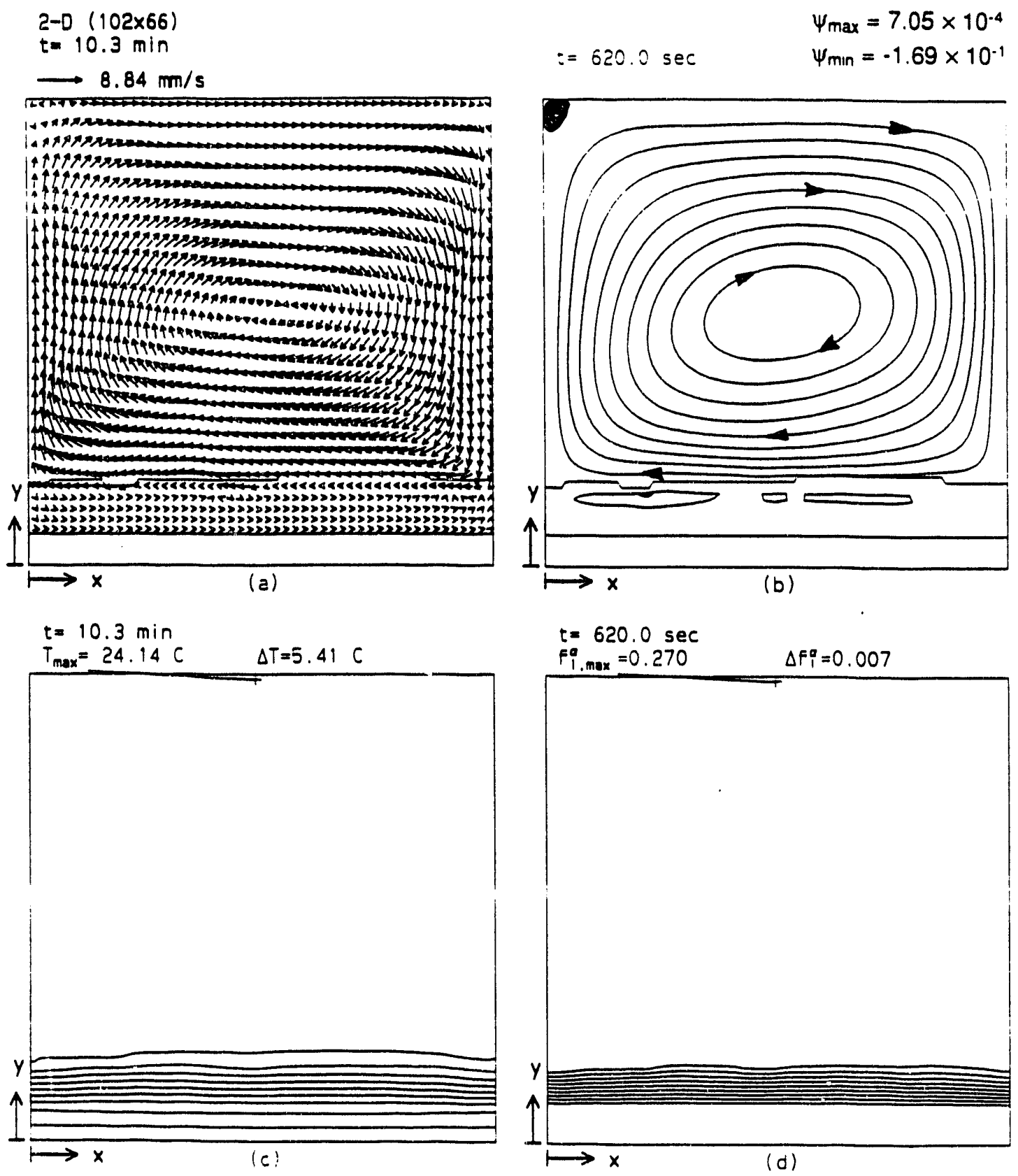

Figure 6.19 Predicted solidification behavior of case NUDS $4 \mathrm{R}$ at $620 \mathrm{~s}\left(\theta=22.5^{\circ}\right)$ : (a) velocity vectors, (b) streamlines, (c) isotherms, and (d) liquid isocomposition. 

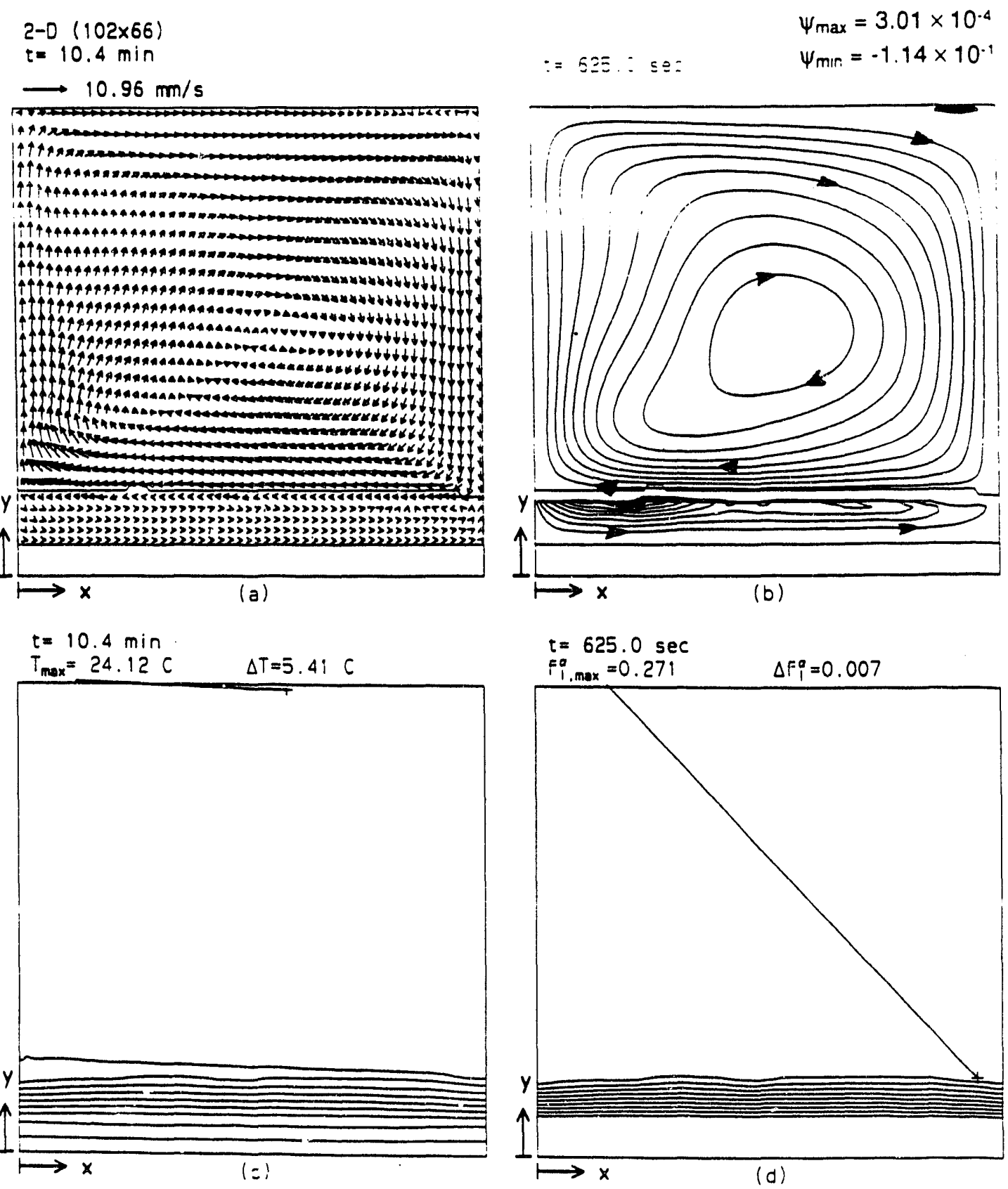

Figure 6.20 Predicted solidification behavior of case NUDS $4 \mathrm{R}$ at $625 \mathrm{~s}\left(\theta=38.4^{\circ}\right)$ : (a) velocity vectors, (b) streamlines, (c) isotherms, and (d) liquid isocomposition. 

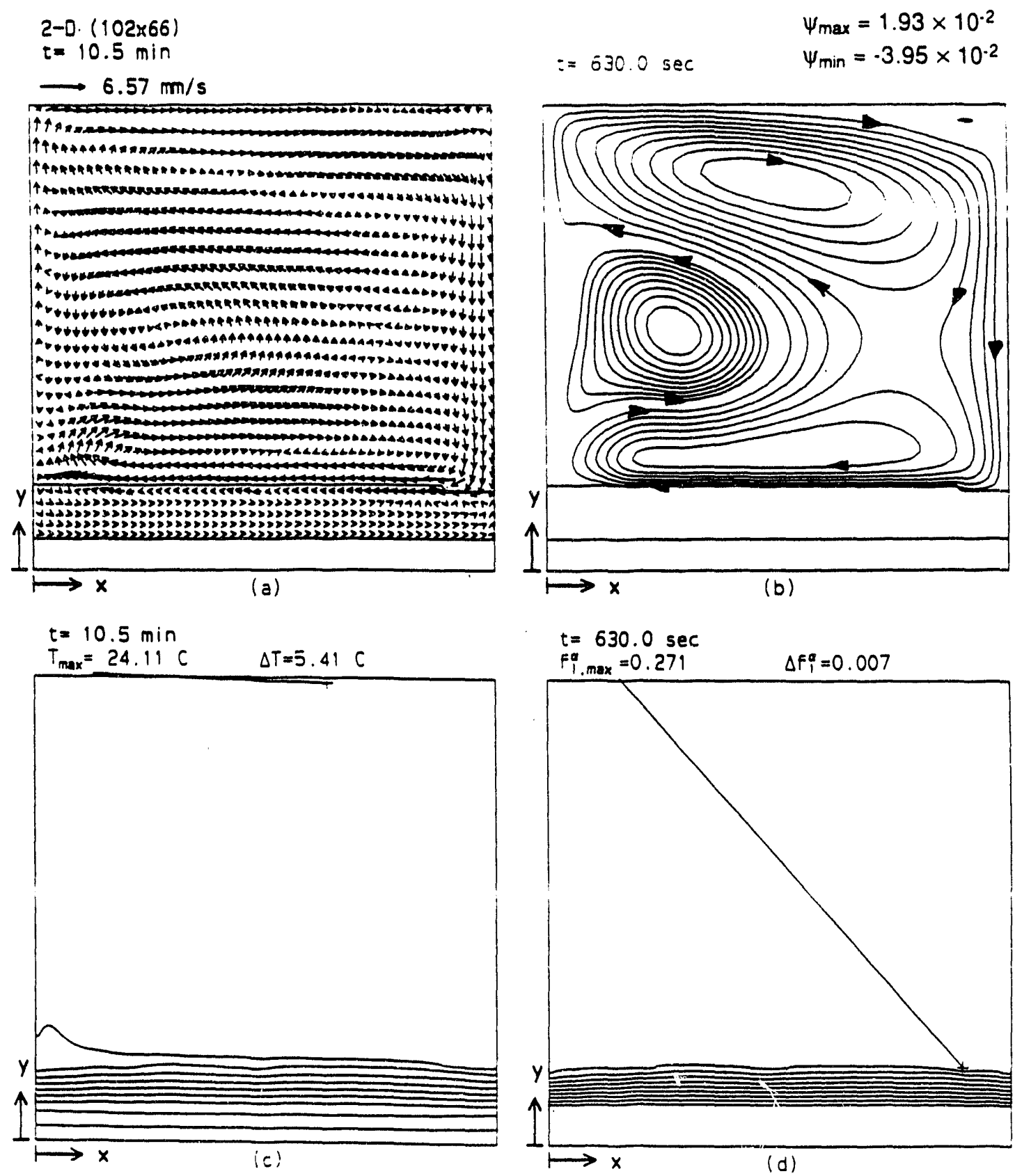

Figure 6.21 Predicted solidification behavior of case NUDS $4 \mathrm{R}$ at $630 \mathrm{~s}\left(\theta=45^{\circ}\right)$ : (a) velocity vectors, (b) streamlines, (c) isotherms, and (d) liquid isocomposition. 


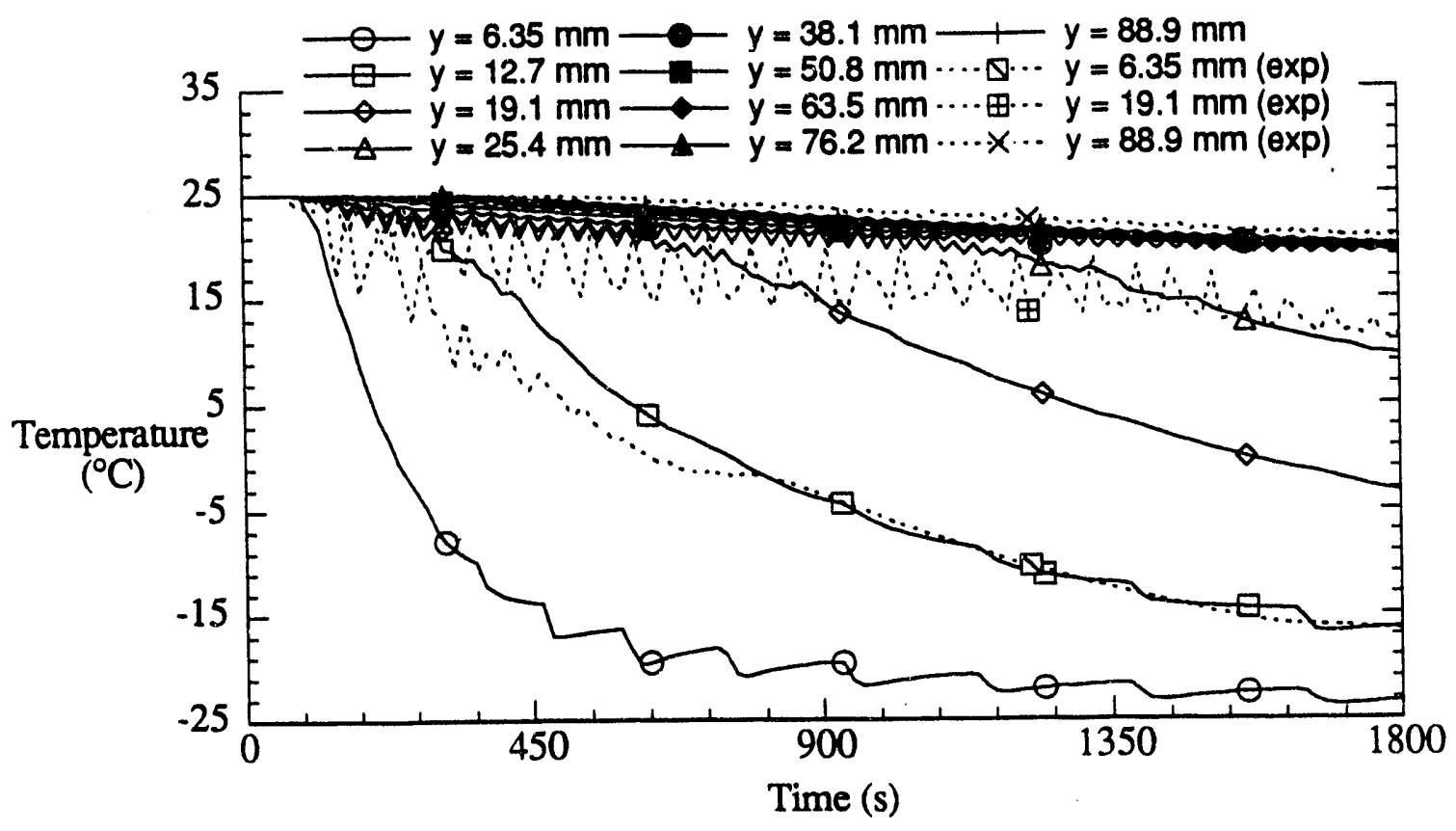

(a)

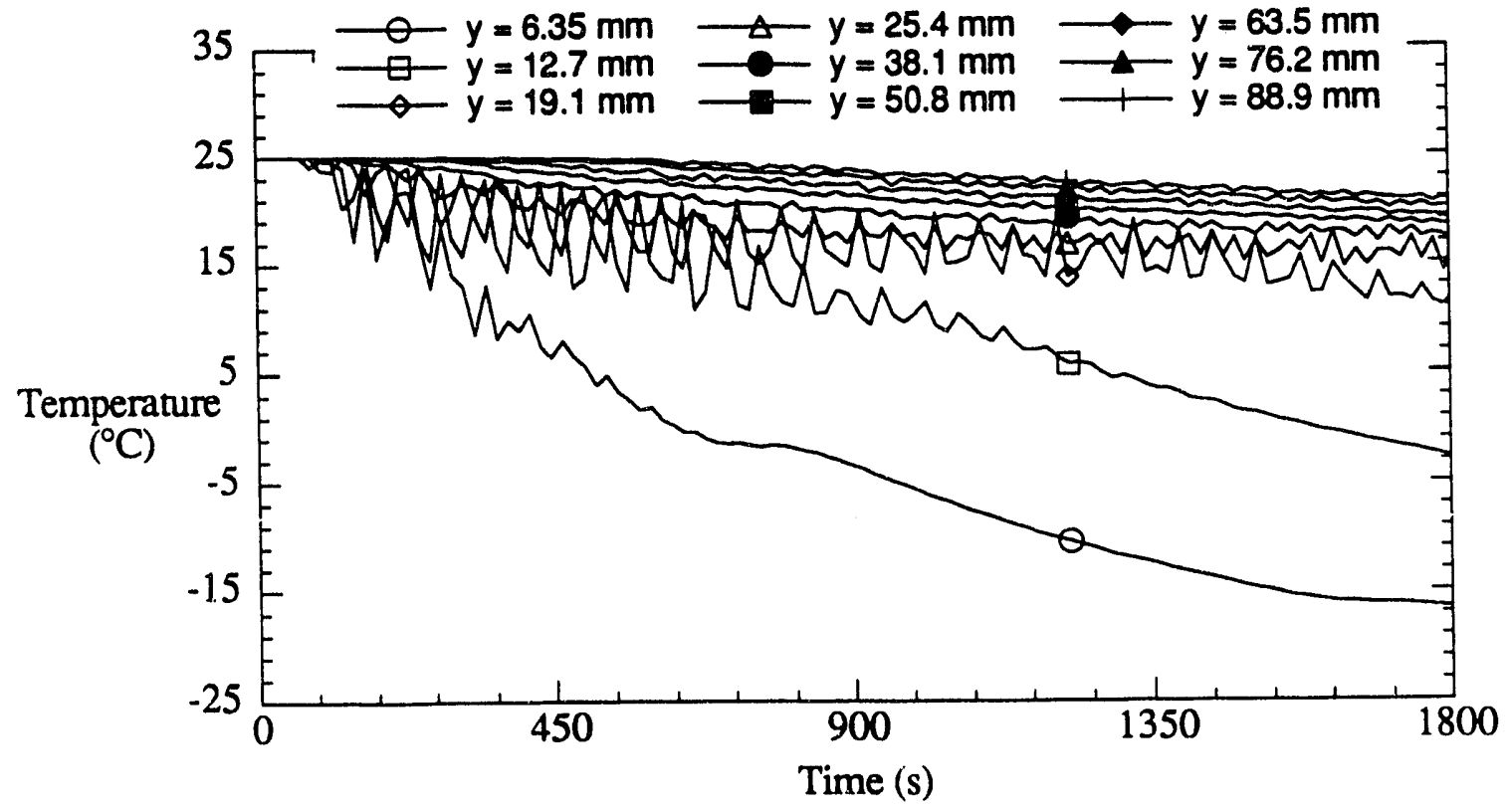

(b)

Figure 6.22 Temperature histories for case UDS 4R: (a) numerical predictions and (b) experimental results. 
the melt significantly exceeded measured variations, and 2) measured temperature fluctuations just above the liquidus interface were more severe than the predictions. Because the cold wall temperature used in the simulation was much lower than the experimental value, one would expect the simulation to underpredict the experimental cooling curves, and for the lower regions of the cast, this is indeed the case. However, within the melt, measured temperatures were far more stratified and a bit cooler than those of the nearly isothermal melt predicted by the simulation. This enhanced cooling is attributed to salt fingering at the liquidus interface, which augmented thermal and solutal transport from the mushy region to the melt. Through increased heat transfer, the fingering also tended to stratify melt temperatures. During the later stages of the simulation, when the production of water-rich fluid decreased, predicted transport between the mushy region and melt was not as vigorous as that suggested by the measurements. Therefore, the model's inability to account for salt fingering reduced its prediction of energy and species transfer from the mushy region to the melt. The result was higher temperatures and nearly isothermal conditions in the melt during later stages of the simulation.

The other problem with the simulation was that predicted temperature fluctuations in Figure 6.22a were far less robust than the actual fluctuations. Unfortunately, predictions were sampled every 15 seconds, which is numerically in perfect phase with the rocking rate. The temperature fluctuations therefore suffer from an alias of the kind discussed in Chapter 5, and the fluctuations in Figure 6.22a do not provide actual peak values in the temperature. To get an indication of the true temperature history predicted by the simulation, one rocking cycle from 900 to 960 seconds was simulated in which the temperatures were recorded every second. Figure 6.23 shows temperature histories in the melt from this computation, and Figure 6.24 shows flow conditions at selected times during the interval. At $900 \mathrm{~s}$, when the section was tilted to $\theta=-45^{\circ}$ (Fig. 6.11), the strong thermal boundary layer which developed along the liquidus interface was vigorously 


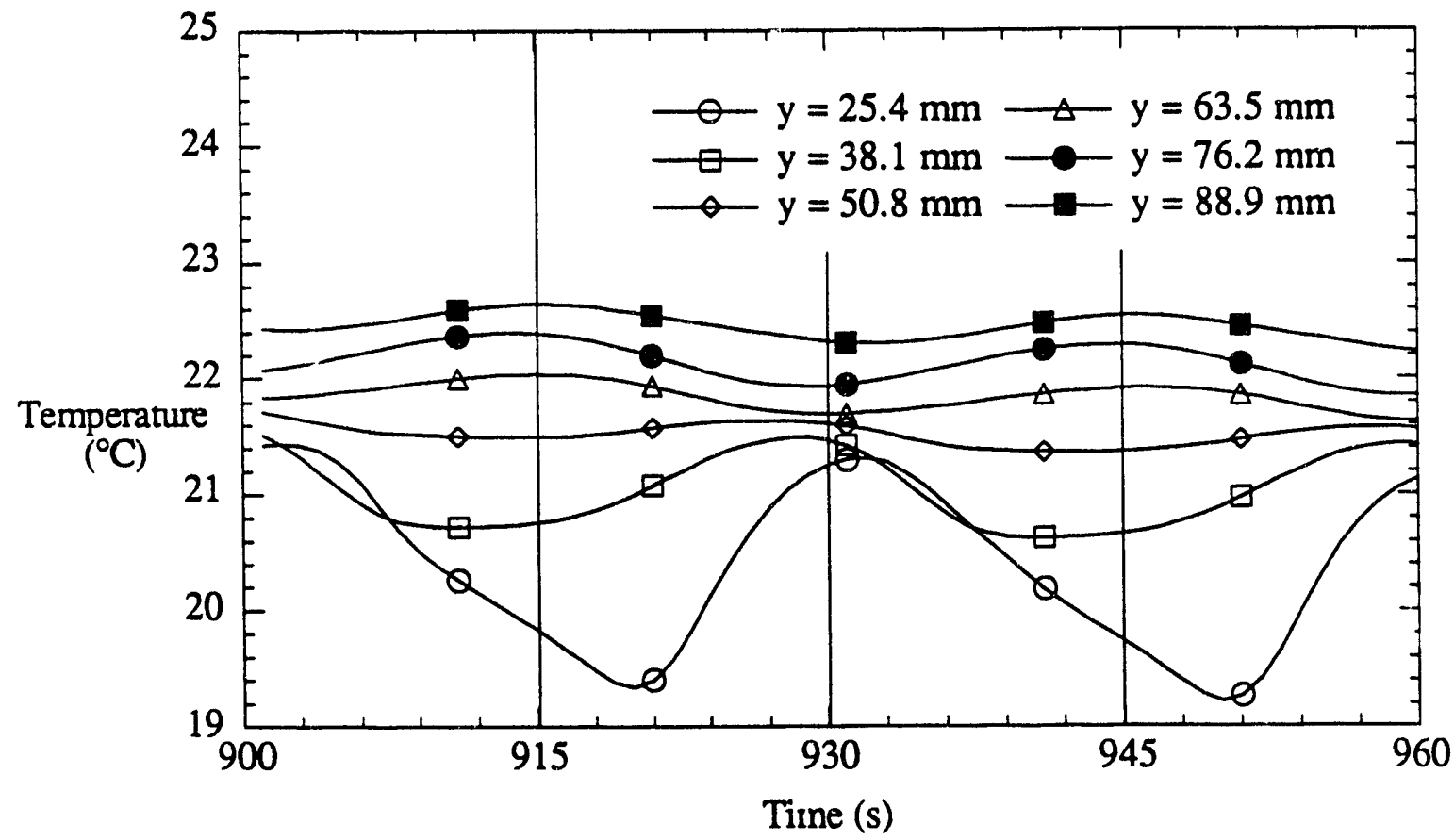

Figure 6.23 Predicted temperature histories in the melt of NUDS 4R over one rocking cycle. 

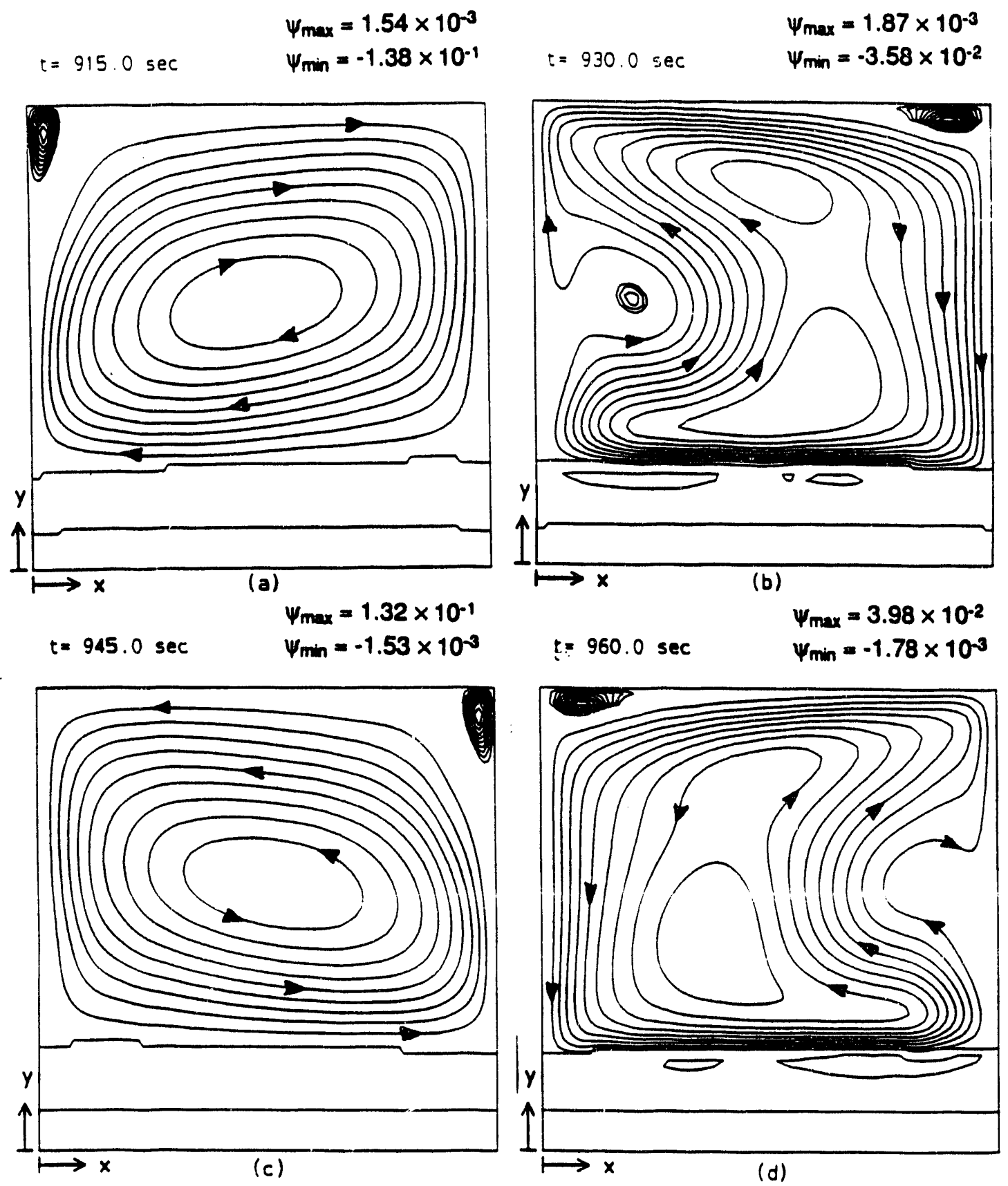

Figure 6.24 Streamline patterns from NUDS $4 R$ at (a) $915 \mathrm{~s}$, (b) $930 \mathrm{~s}$, (c) $945 \mathrm{~s}$, and (d) $960 \mathrm{~s}$ 
exchanging the chilled fluid from the growth front with warm fluid from the melt, thereby reducing the overall vertical temperature gradient. As the box then began to rock back toward the vertical position, inertial effects drove the fluid within the cavity in a clockwise manner, thus opposing the existing thermal buoyancy and reducing the strength of ti.e liquidus washing. At 915s (Fig. 6.24a), the now vertical test section contained a dominant, inertially driven cell in the melt. The velocities in the cell were relatively low and more importantly, the shearing was minimal along the liquidus interface. With decreased convection in the cavity, energy was more easily conducted away to the mushy region, and the temperature along the growth front steadily dropped. Consequently, without the strong supply of chilled fluid from the lower cavity, temperatures in the upper melt rose slightly. As the box rocked past the vertical, a thermally driven boundary layer, augmented by inertial convection, quickly developed at the growth front, sweeping away the chilled fluid and replacing it with warm fluid from the melt. Therefore, the temperature at $y=25.3 \mathrm{~mm}$ quickly rose and the temperature at $y=88.9 \mathrm{~mm}$ dropped, and the overall vertical temperature gradient in the melt was decreased (Fig. 6.23). Figure 6.24b shows the vigorous melt convection which produced near isothermal conditions in the cavity. Past 920s, the flow conditions in the section mirrored those produced from 900 to 930 s (Fig. $6.24 \mathrm{c}$ and d), thus producing the cyclical temperature profiles presented in Figure 6.23. The vertical lines in Figure 6.23 represent those sampling locations used in producing the overall predicted temperature histories (Fig. 6.16a), and the figure clearly shows how temperature variations can be under-reported. However, for $\mathrm{y}=25.4 \mathrm{~mm}$, temperature fluctuations shown in Figure 6.23 are still about $80 \%$ smaller than fluctuations determined experimentally. Hence, this alias alone does not explain why actual temperature fluctuations are as much as five times larger than predicted fluctuations.

The large fluctuations recorded experimentally can be traced to two important phenomena which the model fails to consider. As reported in Chapter 5, fluid motion 
along the liquidus interface is strongly affected by three-dimensional vortices generated by boundary layers on the front and back viewing plates. These vortices increase mixing above the growth front, augmenting the exchange of warm and cold fluid from the melt and mushy regions, thereby contributing to large temperature fluctuations. The other neglected phenomenon in the simulation is undercooling. Temperatures as low as $13^{\circ} \mathrm{C}$ were recorded in the liquid ahead of the growth front, clearly showing the presence of undercooling. In the experiment, temperatures above the liquidus interface fluctuate between those characteristic of undercooling and the bulk melt temperature, as warm and cold fluid is advected between these regions. However, because the model assumes thermodynamic equilibrium, once a location reaches the liquidus temperature (approximately $18^{\circ} \mathrm{C}$ ), a mushy region grows, damping advection in that control volume and reducing fluctuations. Therefore, the model never does allow large temperature gradients to develop ahead of the growth front, thereby creating large fluctuations in temperature due to advection.

\subsection{Side and Bottom Wall Chilling}

The conditions associated with the numerical simulation NMDS 1 are shown in Figures $6.25-6.28$. Overall, the simulation captured important trends related to flow phenomena, macrosegregation, and energy and species transport. However, the model was acutely vulnerable to errors arising from the omission of small scale double-diffusive convection (salt fingers), thermodynamic nonequilibrium in the melt, and the existence of an active slurry.

As with the experimental results, early in the simulation flow within the melt was dominated by thermally driven boundary layers which descended along the growth fronts on the side walls (Fig. 6.25b). Chilled as they descended along the liquidus interface, the boundary layers enhanced growth at the bottom of the mushy region. Due to the absence 

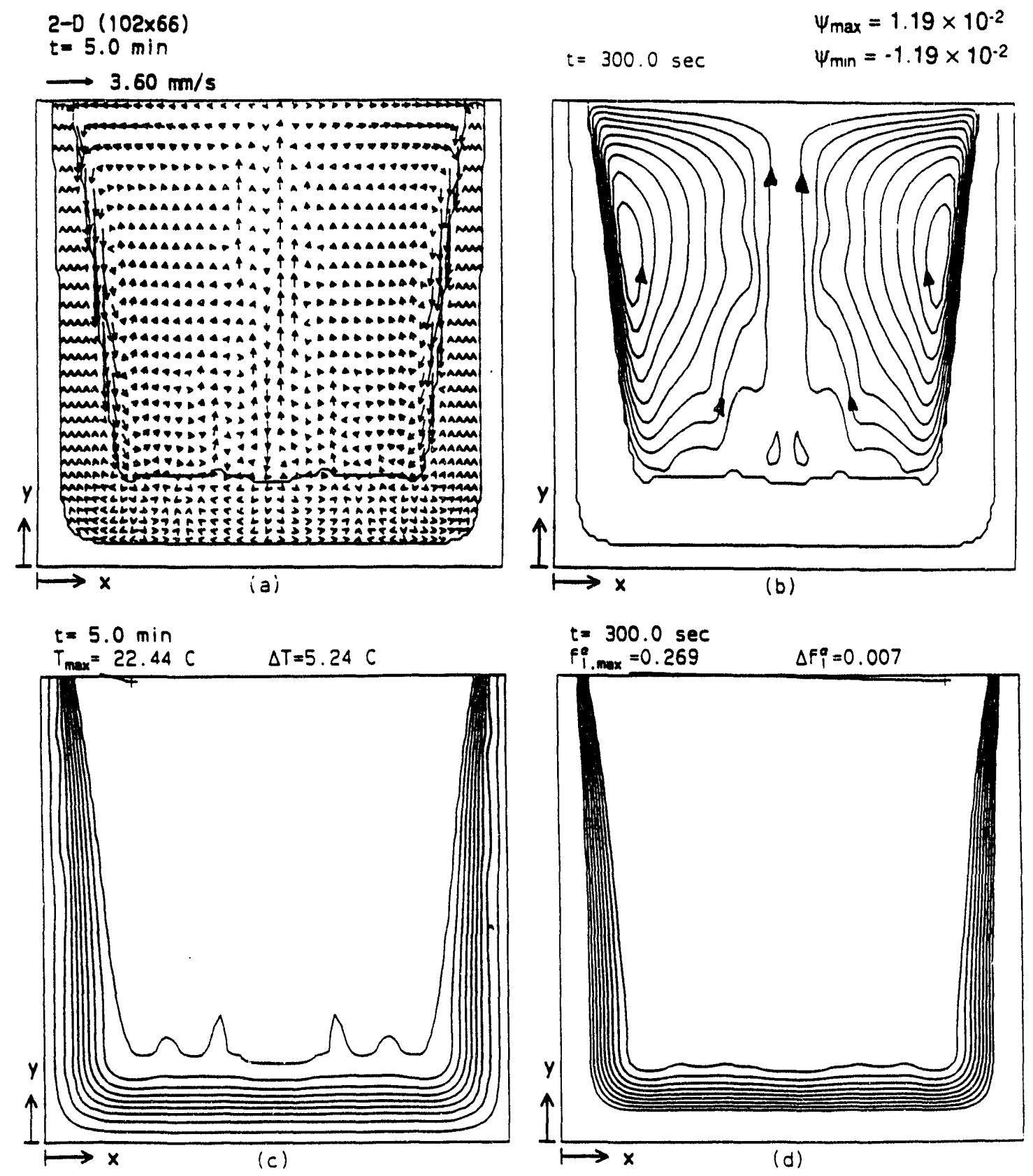

Figure 6.25 Predicted solidification behavior of case NMDS 1 at 300 s: (a) velocity vectors, (b) streamlines, (c) isotherms, and (d) liquid isocomposition. 


\section{$2-D(102 \times 66)$ \\ $t=10.0 \mathrm{~min}$}

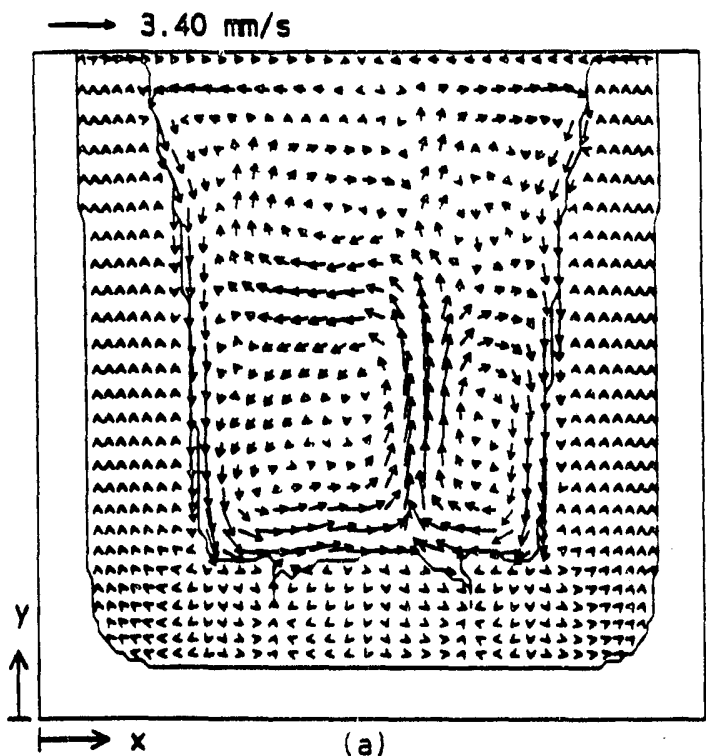

$t=10.0 \mathrm{~min}$

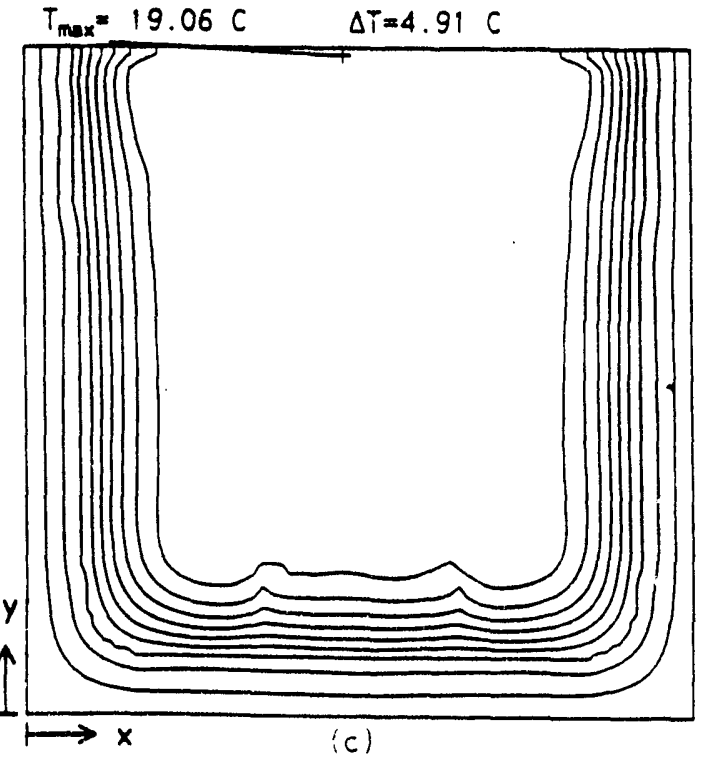

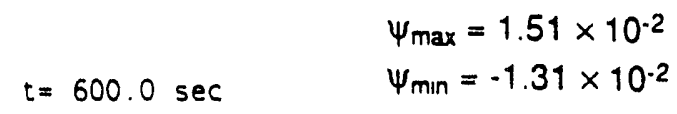
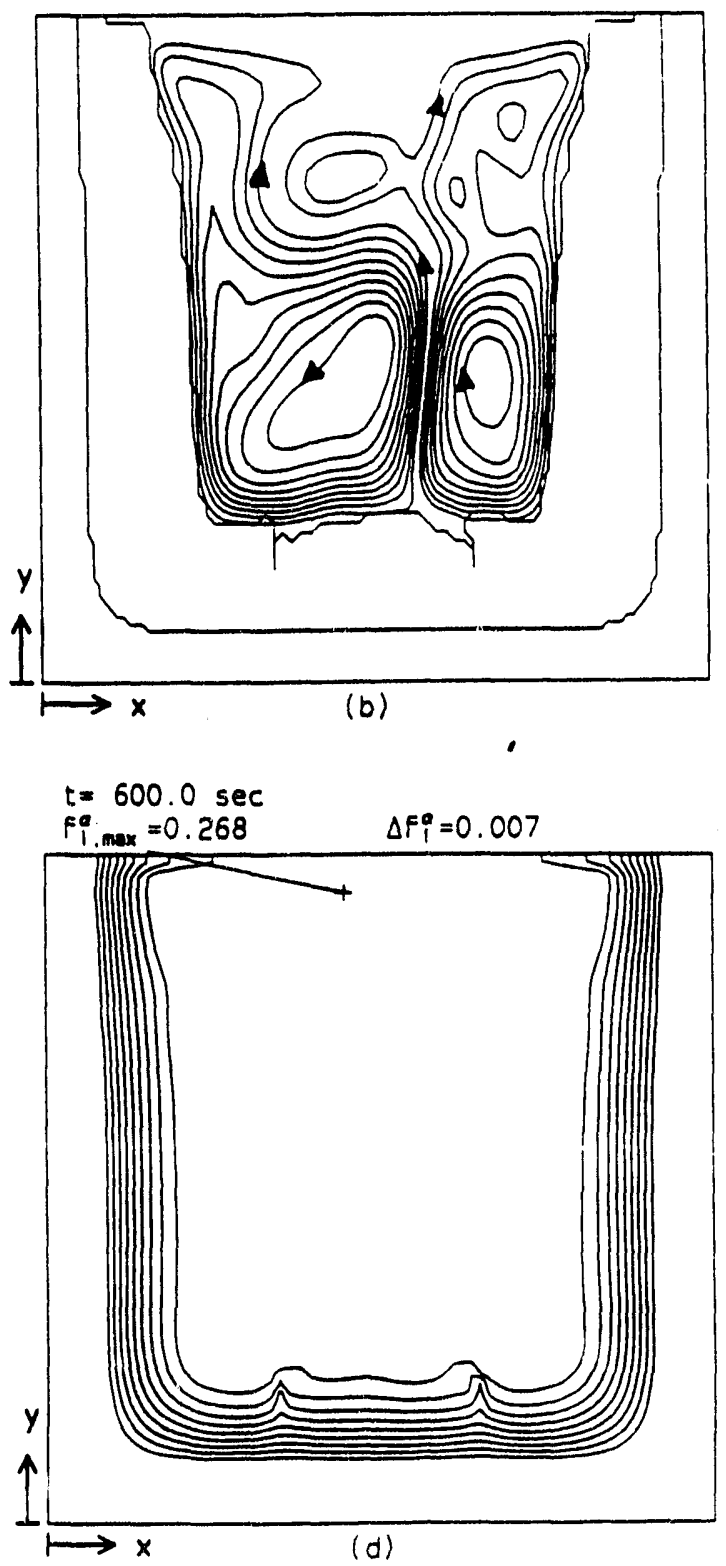

Figure 6.26 Predicted solidification behavior of case NMDS 1 at $600 \mathrm{~s}$ : (a) velocity vectors, (b) streamlines, (c) isotherms, and (d) liquid isocomposition. 

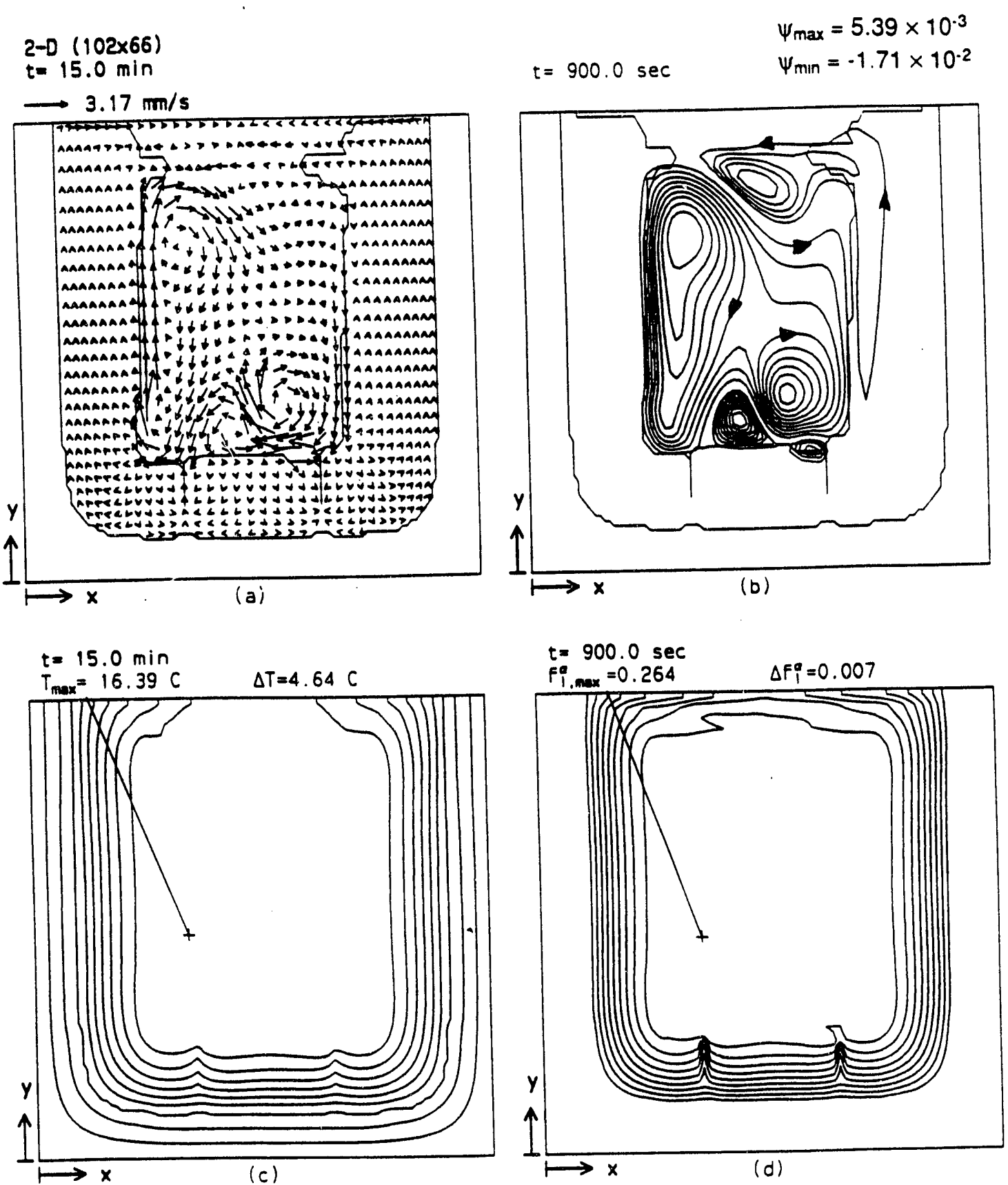

Figure 6.27 Predicted solidification behavior of case NMDS 1 at 900 s: (a) velocity vectors, (b) streamlines, (c) isotherms, and (d) liquid isocomposition. 

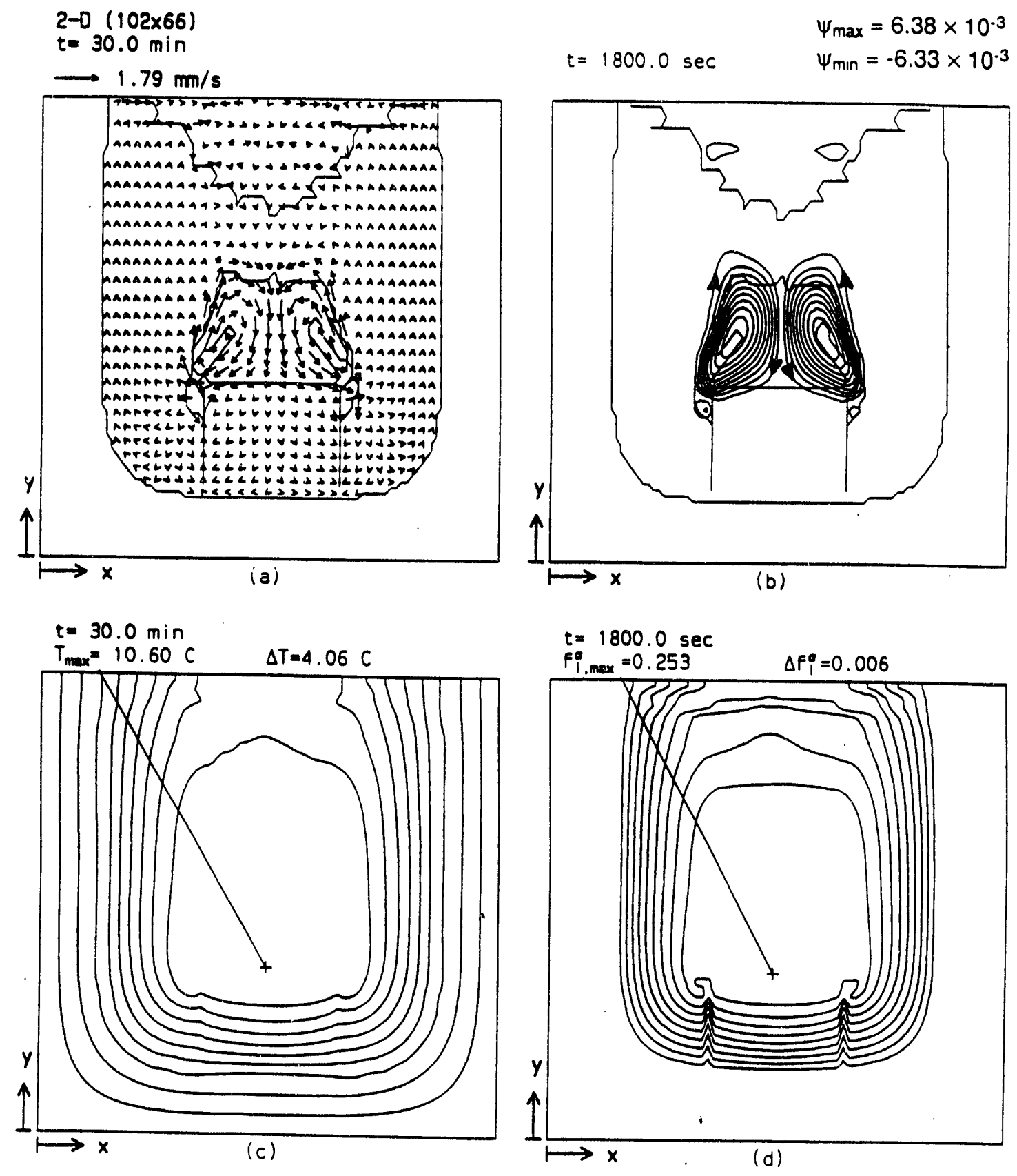

Figure 6.28 Predicted solidification behavior of case NMDS 1 at $1800 \mathrm{~s}$ : (a) velocity vectors, (b) streamlines, (c) isotherms, and (d) liquid isocomposition. 
of a slurry zone in the predictions, the mushy region was relatively planar along the bottom wall. In contrast, experiments showed a dense slurry which descended to the bottom mushy region, forming an uneven, jagged liquidus interface (Fig. 4.15d). Two large, thermally driven counter-rotating convection cells developed in the melt, and disturbances in the lower central regions of the cells were induced by solutally buoyant salt fingers (Fig. $6.25 \mathrm{~b})$. In general, overall flow conditions in the cast at five minutes appeared similar to experimental conditions.

At ten minutes into the simulation, two channels had formed along the bottom wall mush, each rejecting solutally light fluid (Fig. 6.26b). The fluid emanating from both channels coalesced to form a single plume which strongly influenced convection in the melt. The absence of many distinct plumes in the simulation, like those observed experimentally, can again be attributed to the coarse grid. Within the bottom mushy region, flow was entrained into the channels, much like that predicted for the static UDS simulation, while fluid in the side wall mushy region ascended through the porous medium, eventually escaping at the top of the cavity. This ejection of water-rich fluid formed a crystal free pocket at the top of the mushy region, and both the isotherms and isocomps showed the pooling of cold, water-rich fluid in the upper corners of the melt (Fig. 6.26c and d).

The upwelling of cold fluid from the side wall mushy region continued, and at 15 minutes, the pocket of cold, water-rich fluid had extended across the top of the melt (Fig. $6.27 \mathrm{c}$ and $\mathrm{d}$ ). Under the assumption of thermodynamic equilibrium, the mushy region began to extend outward in the upper region of the melt (Fig. 6.27b). Although the mass fraction of solid was small in these areas, on the order of $0.01 \%$, velocities were effectively damped in the upper regions of the melt. Plumes emanating from the bottom wall mushy region remained strong, forming complex flows with irregular vortices in the lower melt. 
At the conclusion of the simulation, 30 minutes, the mushy region growing from the side walls had bridged across the cavity, effectively separating the melt into an upper and lower part (Fig. 6.28b). The large side wall mushy region ejected cold, water-rich fluid into the upper melt producing flow conditions similar to that of the double-diffusive convection cells which formed during the experiments. Due to the ejection of water-rich fluid, horizontal channels began to form in the mushy region at the top of the cast, indicating the development of A-type segregates in the side wall mush. Flow in the lower melt region was still dominated by robust plumes rising from deep channels, But was strongly inhibited by the growing mushy region. As was observed experimentally, the predicted temperature and composition plots show that cold, water-rich fluid rejected by the side wall mush coalesced in the top of the cast (Fig. 6.28c and d). Consequently, the warmest, highest concentration fluid was forced to the bottom of the melt.

The overall macrosegregation at 30 minutes is shown in Figure 6.29. The most severe segregation was associated with channels in the bottom wall mushy region. Essentially freckle-type segregates resulted from channels in the bottom mush and were characterized by localized salt-rich regions in the adjacent crystalline structure. Within the side wall mushy region, the continuous upwelling of fluid through the course of the simulation produced water-rich areas or bands which are otherwise referred to as A-type segregates. In addition, the near eutectic pools of fluid that collected in the upper corners of the melt remained as water-rich segregates.

In general, the simulation overpredicted temperatures in the cast, despite the colder boundary conditions used in the model. With respect to variations in the vertical direction, predicted melt temperatures were warmer and less stratified than experimental results early in the simulation (Fig. 6.30). As seen for case NUDS $4 R$, salt fingers produced by the growing mushy region augmented convective transport at the liquidus interface, yielding melt temperatures which were lower than predictions. However, the model successfully 


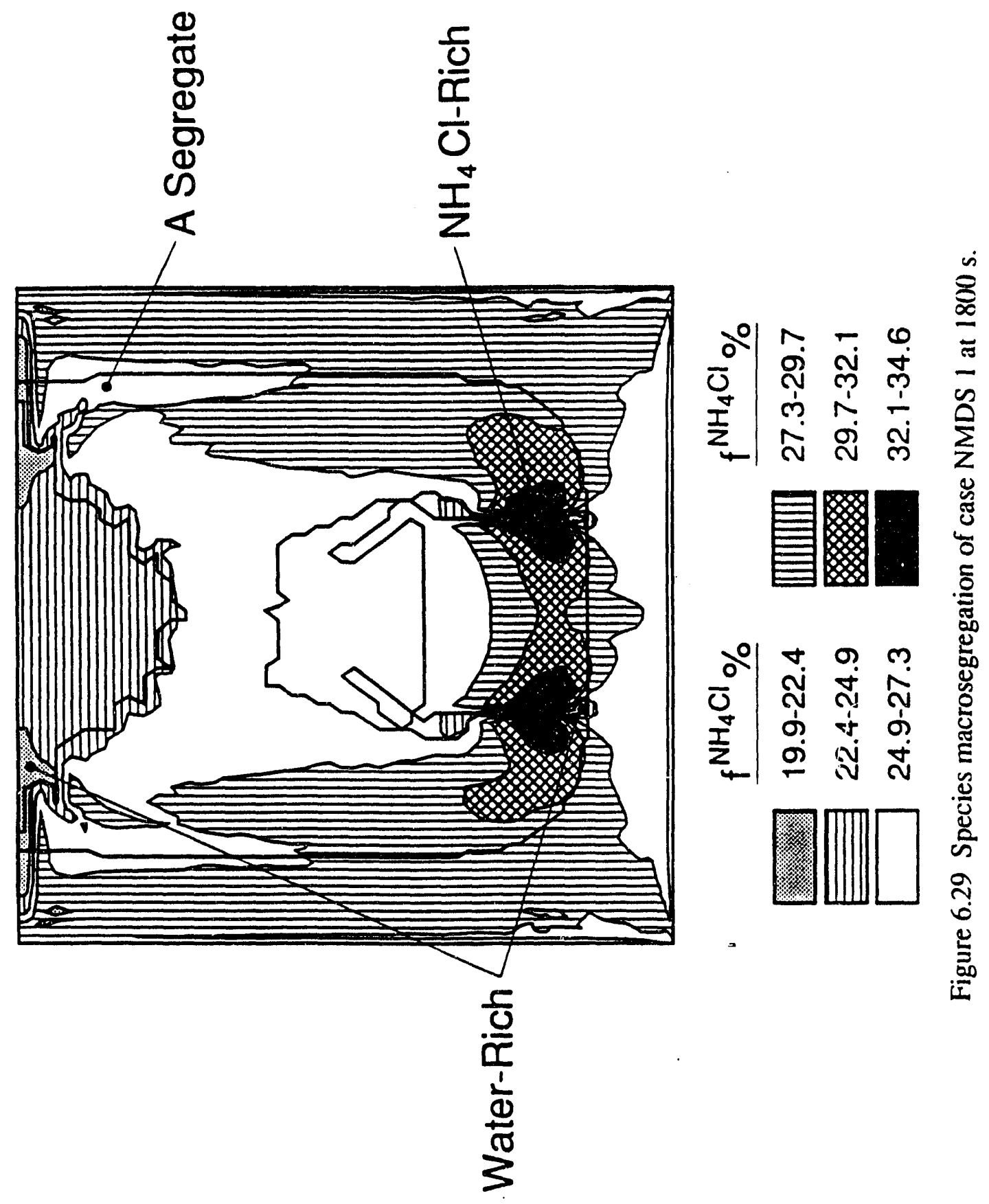




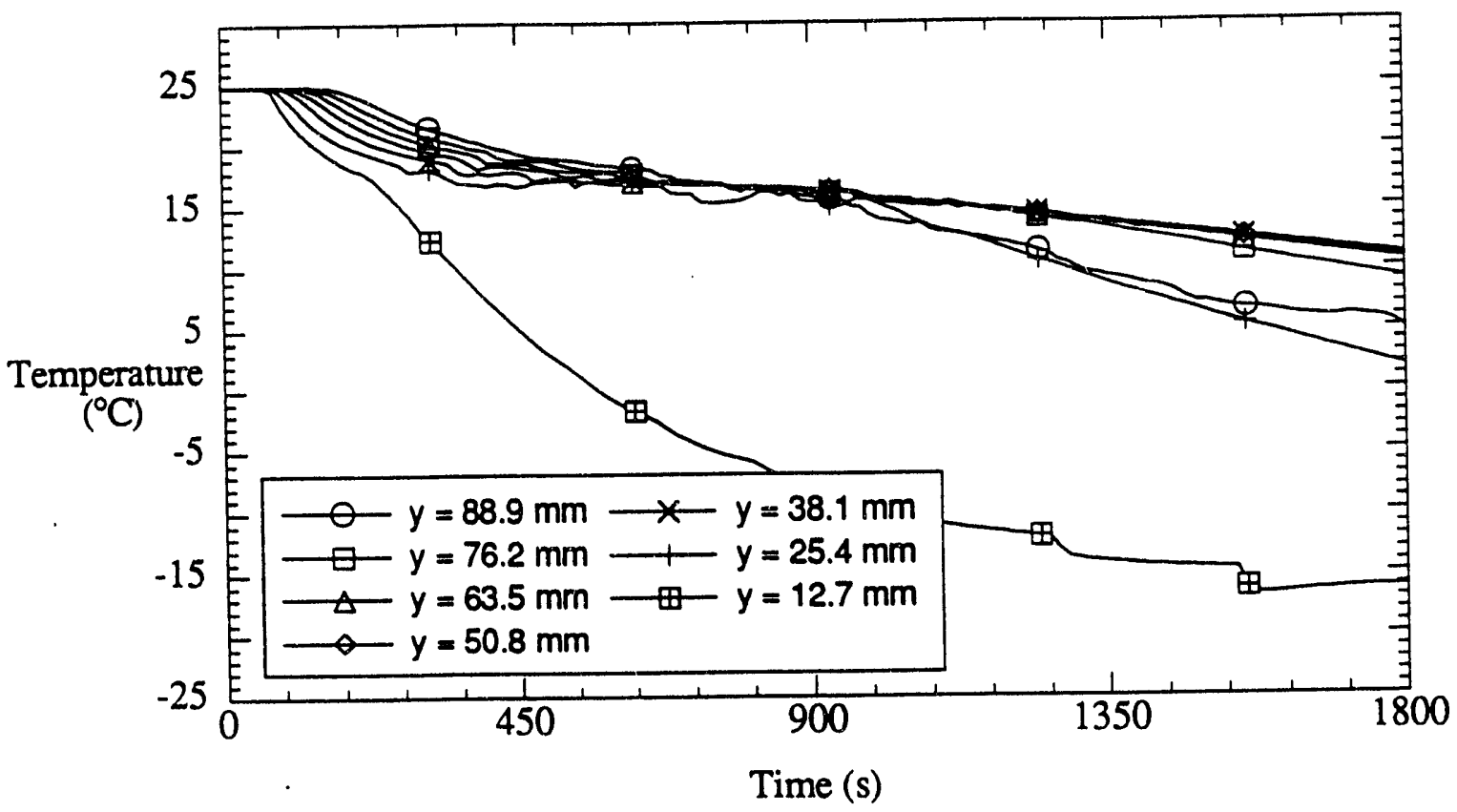

(a)

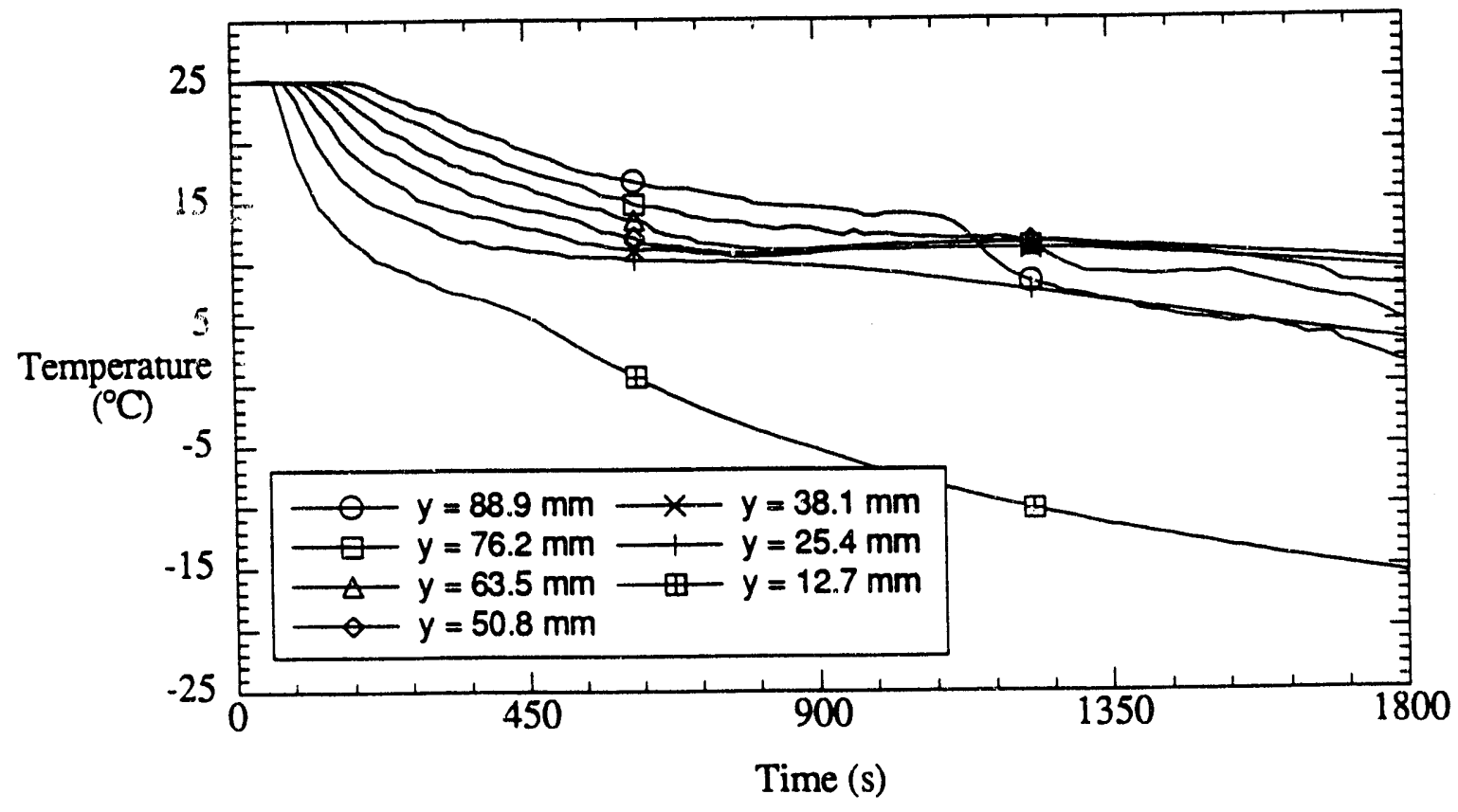

(b)

Figure 6.30 Temperature histories along vertical thermocouple array located at $\mathrm{x}=38.1 \mathrm{~mm}$ for case MDS 1 : (a) numerical predictions and

(b) experimental results. 
predicted the thermal stratification which developed in the melt toward the end of the simulation. At 30 minutes, the model predicted that the region from $y=38.1 \mathrm{~mm}$ to 63.5 $\mathrm{mm}$ was the warmest in the melt and that the total temperature difference between the high and low meit temperatures was $9.0^{\circ} \mathrm{C}$. Similarly, the experimental results showed that the region from $y=38.1 \mathrm{~mm}$ to $63.5 \mathrm{~mm}$ was indeed the warmest and that the stratified temperature difference was $8.5^{\circ} \mathrm{C}$.

A direct comparison of predicted and measured temperatures at different spanwise locations is presented in Figure 6.31, and again the predictions exceed the experimental results. However, the predicted liquidus interface reached $x=12.7 \mathrm{~mm}$ approximately ten minutes before the real interface did. When a temperature location is overgrown by the liquidus interface, $D^{\prime}$ Arcy damping of the mushy region shields the location from advective mixing in the melt, which normally maintains nearly uniform temperature in the horizontal direction. Hence, passage of the liquidus interface can be deduced from the temperature histories by noting the time at which a significant departure from isothermal behavior occurs. In this case, the predicted liquidus interface reached $x=12.7 \mathrm{~mm}$ approximately 10 minutes before and at a $5^{\circ} \mathrm{C}$ higher temperature than the actual interface. The delay in the progression of the liquidus interface can again be attributed to undercooling in the melt of the experiment. Assuming thermodynamic equilibrium, the model over-predicted the location of the growth front. However, disregarding the model's omission of undercooling and salt fingering, predicted cooling trends are reasonably consistent with experimental results.

Finally, the predicted liquid species composition was compared to experimental results (Fig. 6.32). Again, although the predicted trends are consistent with measurements, the model, through the omission of salt fingering, overpredicted concentrations in the melt. 


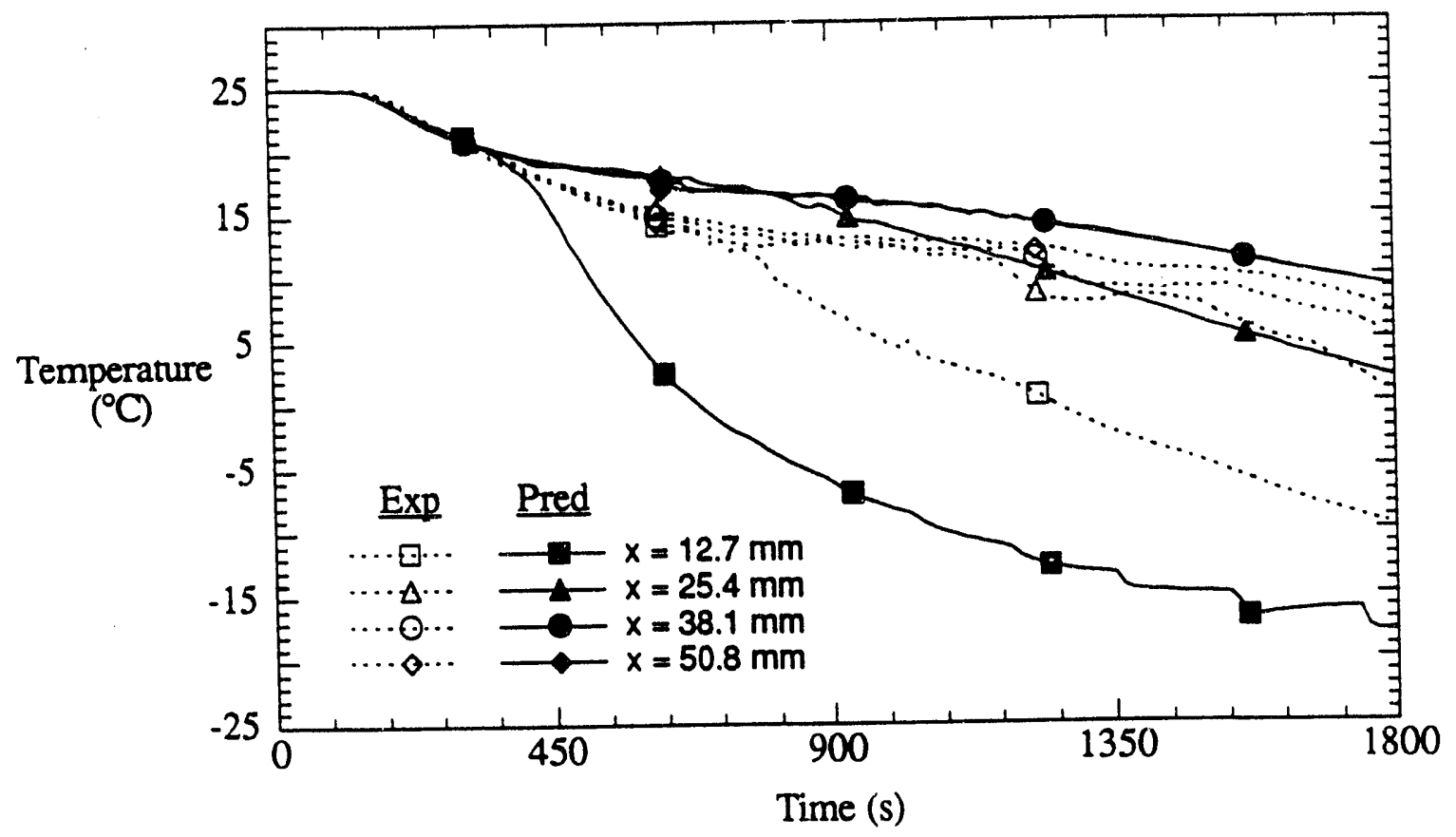

(a) Upper Array

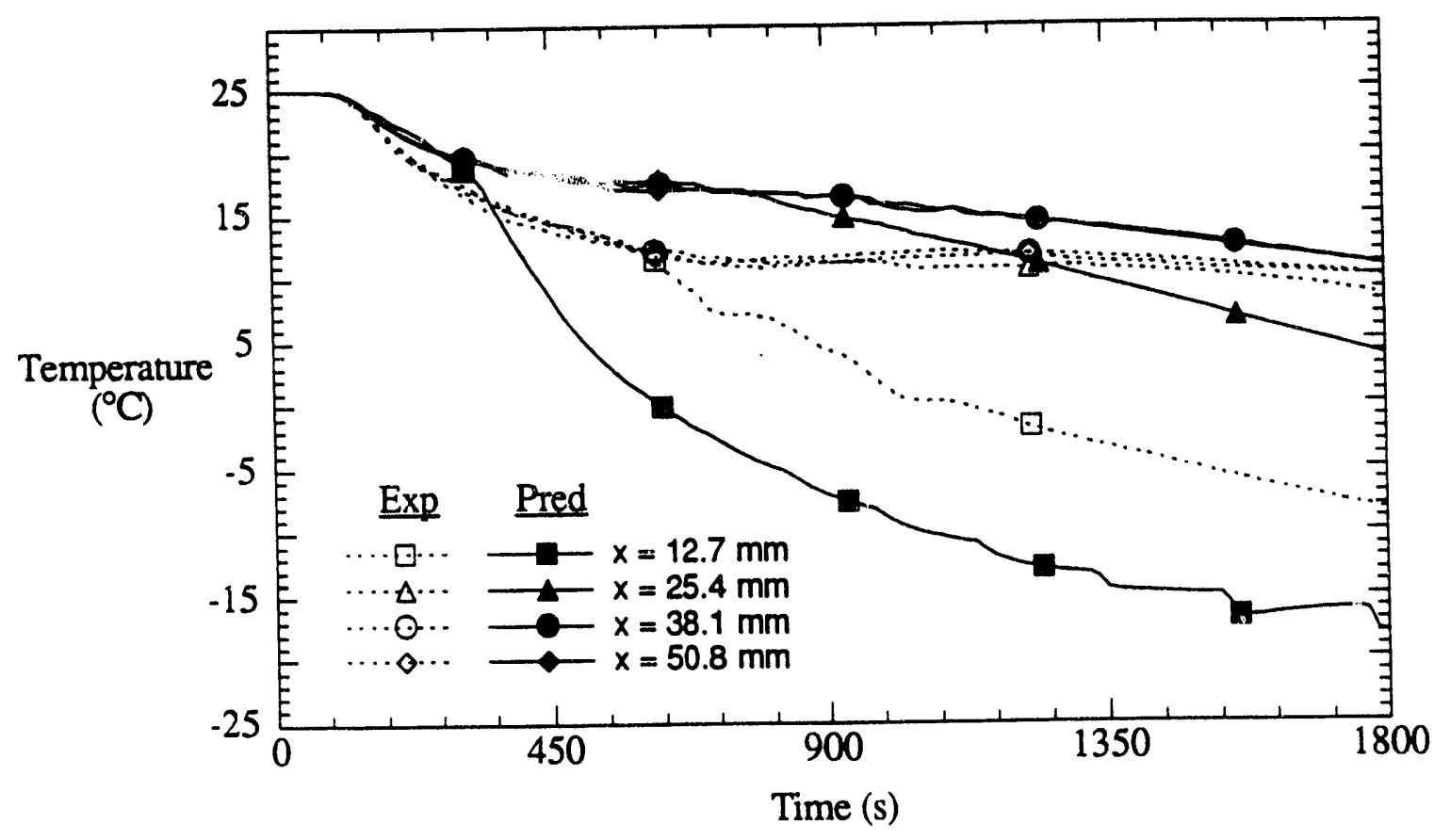

(b) Lower Array

Figure 6.31 Comparison of predicted and experimental temperature histories for case MDS 1 at (a) $y=76.2$ and (b) $y=50.8 \mathrm{~mm}$. 


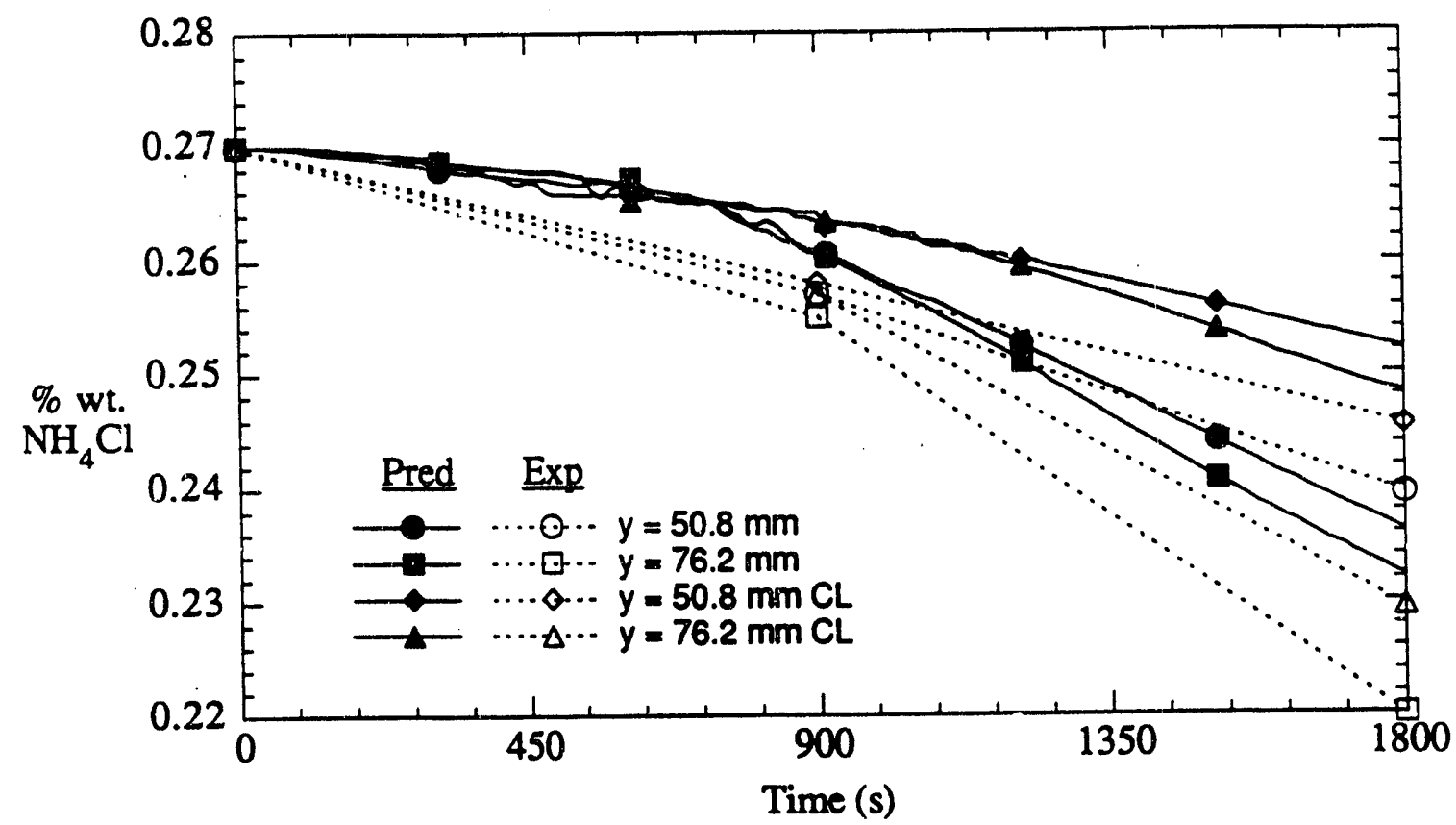

Figure 6.32 Comparison of predicted and experimental liquid composition of ammonium chloride for case MDS 1 . 


\section{CHAPTER 7 - CONCLUSIONS AND RECOMMENDATIONS}

\subsection{Conclusions}

An experimental and numerical investigation of the directional solidification of binary alloys has been undertaken. By studying the behavior of freezing aqueous ammonium chloride, a popular low temperature metal alloy analog, the goals of the project were to gain a deeper understanding of the solidification process $a$, ultimately, to improve the qualify of solidified products by suppressing or eliminating imperfections which often develop during solidification. Macrosegregation is one such imperfection. Produced by convesive transport of species in the melt and mushy regions of a solidifying ingot, freckles, A-segregates, and banded segregation are all common manifestations of macrosegregation. Two small, rectangular test sections were constructed, each allowing visual observation of the solidification process through shadowgraph, particle tracking, and dye injection techniques. In an attempt to suppress macrosegregation, the solidifying ingots were subjected to a slow, oscillatory rocking motion which was intended to enhance mixing in the melt and to inhibit convective flows which ordinarily lead to macrosegregation.

\subsubsection{Unidirectional Solidification}

A number of experiments and numerical simulations were performed for unidirectional solidification (UDS), and under static conditions, the dependence of macrosegregation on double-diffusive salt fingering was apparent. 'Produced by the release of solute deficient fluid from the growing mushy region, salt fingering is characterized by water-rich ascending columns of fluid adjacent to descending salt-rich columns of fluid. 
have the thick overgrowth nor an associated volcano. In addition, the numerical simulations showed that overall macrosegregation was improved with rocking.

Due to the presence of a dense slurry generated early in the rocking experiment, the growth front morphology of the rocking UDS cast, though colum-ar, was characterized by ranoimly oriented primary dendrites. This condition was in contrast to the static case, wher he growth front was characterized by single crystal grains held together in a rigid matrix. It is not clear whether the randomly oriented dendrites of the rocked cast are weaker than statically grown grains, nor is such a question within the scope of the present investigation. However, the question merits further attention in future research

With rocking, the numerical model was again able to predict key trends and to provide a more detailed understanding of macrosegregation and mushy region flow conditions which could not be otherwise determined. However, the model again failed to provide good agreement with measured quantities. By neglecting the presence of an undercooled fluid above the growth front, the model underpredicted thermal gradients in the melt and convective transport in the system. Also, without a way to account for the small scale mixing associated with salt fingering, the model underpredicted energy and species transport across the liquidus interface. Finally, in assuming two-dimensional flow conditions, the model failed to account for significant three-dimensional effects which characterized the experiments.

Within the limited parametric study conducted in the investigation, conditions which minimized channel formation were found to be associated with rocking at one cycle per minute at an amplitude of $45^{\circ}$. This finding suggests the existence of an optimal rocking condition. However, the optimum could depend on growth rate, thermal gradients, characteristic lengths, solutal concentrations, or any number of parameters governing system behavior. 
From the moment crystals began to appear, salt fingers rose into and mixed with the melt. Throughout the early stages of UDS, these salt fingers strengthened and organized, competing for the supply of interdendritic fluid produced within the mushy region. The fingers became sufficiently vigorous to break dendrites from the mushy region and to produce a weak slurry in the melt. Drawing fluid from deep within the mushy region, the stronger salt fingers produced upflows which induced a thermodynamic melting point depression, concomitant melting of adjoining salt crystals, and the eventual formation of holes or channels in the porous structure.

The foregoing channels became paths of least resistance for the flow of near eutectic cold fluid from deep within the mushy region, and the fluid rising from the channels formed cylindrical plumes which rose to the top of the cavity. Although the salt fingers and plumes were both manifestations of double-diffusive convection, they are much different in their scope and influence on solidification. The plumes are relatively large scale, robust fluid ejections that are driven by deep, nearly eutectic and highly buoyant interdendritic fiuid. Conversely, the smaller, less developed salt fingers are primarily associated with fluid which is closer to the liquidus interface and of much lower buoyancy.

It was found that channels tended to form at liquidus front locations characterized by large hillock or elevations during the early salt fingering stages. In an attempt to exploit this finding, short, circular fins extending from the bottom chill plate were used to induce channel formation at predetermined locations in the cast. Such a scheme could be used to preferentially locate freckles in an area of the cast which could readily be removed through post-processing or in an area where the freckles would have no deleterious effect on the final product. Though successful in inducing channels, the grain morphology around each fin was not columnar.

Entraining solution from the surrounding mush, flow in the channels sustained the development of salt-rich sheaths in the mushy region around the channels. Eventually, the 
channels were overgrown by the advancing solidus front, thereby becoming filled with eutectic material and completing the formation of freckles. Additional flow phenomena associated with UDS included the development of double-diffusive convection cells in the melt, planar and helical oscillations in the plumes, and wisps of falling fluid from bends in meandering plumes.

A numerical simulation of the static solidification experiment predicted the formation of channels and resulting macrosegregation patterns. Although the simulation could not provide good agreement with measured field variables, like temperature and liquid composition, it did predict the key trends related to these variables, as well as important information about interdendritic fluid flow and growth front morphology. The inability to obtain good quantitative agreement is attributed to limitations in model assumptions, for example, the assumption of thermodynamic equilibrium. In the static experiments, fluid just above the growth front was observed to be significantly undercooled, with temperatures at the liquid interface significantly lower than those predicted by the equilibrium phase diagram.

When subject to ast illatory rocking motion, flow and morphological conditions associated with the solidification process were significantly altered. Shear layers, which developed at the liquidus interface due to thermal and inertial forces, effectively swept the interface of density perturbations which would ordinarily lead to channels. This beneficial effect was also demonstrated numerically, where the nearly uniform isotherms and isocomps which were predicted in the mushy region testified to the effectiveness of rocking. Although still present, channels which formed under rocking conditions were fewer in number and the size and severity of crystal aggregation around the channel were less severe than those associated with static solidification. While channels formed under static conditions were encircled with a thick overgrowth of dendrites which extended above the growth front like small volcanoes, channels formed during rocking conditions did not 


\subsubsection{Side and Bottom Wall Chilling}

When the solution was statically chilled from both the sides and the bottom, subsequent events included creation of a dense slurry, double-diffusive convection, and an undercooled melt. Dendritic breakage on the side walls and dendritic fracture within the side wall mush established a slurry which descended along the walls and formed large equiaxed regions near the bottom of the cast. Primarily caused by solutal upflow in the side wall mush, crystal erosion eventually created A-segregate type channels which were easily observed and traced with dye injection. Ejection of solutally light fluid established well defined double-diffusive convection cells, which impeded the motion of plumes emanating from volcanoes in the bottom mush, and within the melt cold, water-rich fluid formed above warm, salt-rich fluid. As evidenced by crystal nucleation on thermocouple probes located well within the melt, significant undercooling existed within the liquid region.

The numerical simulation of static cooling from the side and bottom walls predicted trends associated with temperature and composition variations but failed to provide good quantitative agreement with the data. Inability to account for turbulent mixing caused by salt fingers resulted in underprediction of energy and species transport across the liquidus interface, and the assumption of thermodynamic equilibrium predicted a large and physically unrealistic mushy region which altered flow conditions in the melt. In addition, the predicted flow conditions are subject to errors associated with use of a coarse grid and neglect of slurry transport effects.

When the side and bottom wall chilling was subjected to a rocking motion, dendrite breakage from the side wall established a heavy slurry zone which collected on the cavity floor to such an extent as to suppress solidification and salt fingering, thereby eliminating channels from developing in the bottom mush. However, early in the experiment, strong advection along the bottom liquidus interface created a localized recalescence, rare in 
$\mathrm{NH}_{4} \mathrm{Cl}-\mathrm{H}_{2} \mathrm{O}$ systems. In general, higher rocking amplitudes more efficiently mixed the melt, eliminating double-diffusive convection but still allowing for the existence of weak thermal and solutal stratification. Heat transfer from the system was not enhanced by the agitation, and A-segregate channels, as well as overall macrosegregation, were unaffected by the rocking motion.

\subsection{Recommendations}

An important question pertaining to the research is how findings for $\mathrm{NH}_{4} \mathrm{Cl}-\mathrm{H}_{2} \mathrm{O}$ relate to the casting of metal alloys. A number of important distinctions exist between the two systems. Thermal conductivity is much higher in metal systems, and the porous region is generally much less permeable to fluid flow. However, just as in the salt analog, freckles and A-segregates form in metal alloys, and owing to large Lewis numbers, salt fingering and other double-diffusion phenomena probably develop during most metal alloy solidification. When implementing a rocking scheme to reduce macrosegregation in metal alloys, the low Prandtl numbers suggest that less severe rocking amplitudes would be sufficient to remove perturbations from the liquidus interface, since the relatively high thermal conductivity and low viscous drag associated with liquid metals would lead to stronger thermal boundary layers along the growth front. Speculation aside, experiments involving metal alloys are needed to establish specific trends. Although flow visualization cannot be obrained with metal systems, valuable information such as macrosegregation and grain morphology could be collected through analysis of solidified ingots. Such investigations would surely bring current macrosegregation control research closer to industrial needs.

The interdendritic conditions associated with the mushy region is another critical area in need of elucidation. The presence of a slurry was seen to be particularly relevant to morphological conditions leading to channeling and to grain structure. However, it is not 
clear where or how the slurry originates, what forces cause dendrite fracture, or what advective forces are required to break dendrites. In modelling the porous mushy region as well as slurries, these questions gain importance. The behavior of flow though the mushy region also needs attention, where an anisotropic permeability could possibly improve model results.

With respect to controlling macrosegregations in a UDS cast, the rocking scheme shows promise. More detailed parametric studies with varying rocking rates and rocking amplitudes could reveal optimal operating conditions for the rocking scheme. In addition, rocking profiles other than a sinusoidal oscillation may have promise. For example, a mold could be rc ad to its maximum amplitude and held for a period of time to take full advantage of the sweeping motion along the liquidus, before being rocked to the other extreme.

The randomly ordered primary dendrites that developed under rocking UDS conditions may also be eliminated in a simple manner. By holding the test section static in the first 15 minutes of an experiment, the effects of the slurry on grain morphology could be eliminated. After the crystal grains are well established and before channels form, the rocking motion could be started, thereby suppressing channels while still retaining the ordered grain matrix associated with static casts. However, it remains unclear how such a scheme could be applied to metal systems.

Indeed, other convection control schemes could also provide suppression of macrosegregation. Imposed shear layers at the liquidus interface, magnetic damping in metal systems, or new agitation schemes may hold promise in the area. The possibilities are almost limitless.

The numerical model, though already a useful investigation tool, is in need of improvement. Perhaps one of the most useful and simplest additions to the model would be the inclusion of a turbulence capability to account for mixing caused by salt fingering. A 
low energy, long length scale turbulence model could vastly improve characterization of energy and species transport across the liquidus interface. The presence of undercooling in the melt is another large source of error in the model which could be addressed. Finally, as faster computer resources become available, finer grids and full three-dimensional simulations will become practical, thereby improving the model's capability and accuracy. 


\section{REFERENCES}




\section{REFERENCES}

Amberg, G., 1991, "Computation of Macrosegregation in an Iron-Carbon Cast," Int. J. Heat Mass Transfer, Vol. 34, pp. 217-227.

Asai, S. and Muchi, I., 1978, "Theoretical Analysis and Model Experiments on the Formation Mechanism of Channel-type Segregation," Trans. ISIJ, Vol. 18, pp. 90-98.

Beckermann, C. and Viskanta, R., 1988, "Double-Diffusive Convection During Dendritic So'idification of a Binary Mixture," PhysicoChemical Hydrodynamics, Vol. 10, pp. 195-213.

Bennon, W. D. and Incropera, F. P., 1987a, "A Continuum Model for Momentum, Heat, and Species Transport in Binary Solid-Liquid Phase Change Systems: I. Model Formulation," Int. J. Heat Mass Transfer, Vol. 30, pp. 2161-2170.

Bennon, W. D. and Incropera, F. P., 1987b, "A Continuum Model for Momentum, Heat, and Species Transport in Binary Solid-Liquid Phase Change Systems: II. Application to Solidification in a Rectangular Cavity," Int. J. Heat Mass Transfer, Vol. 30, pp. 2171-2187.

Cao, W. Z. and Poulikakos, D., 1990, "Solidification of an Alloy in a Cavity Cooled Through Its Top Surface," Int. J. Heat Mass Transfer, Vol. 33, pp. 427-434.

Chen, C. F. and Turner, J. S., 1980, "Crystallization in a Double-Diffusive System," J. Geophysical Research, Vol. 85, pp. 2573-2593.

Chen, C. F. and Chen, F., 1991, "Experimental Study of Directional Solidification of Aqueous Ammonium Chloride Solution," J. Fluid Mech., Vol. 227, pp. 567-586.

Christenson, M. S. and Incropera, F. P., 1989a, "Solidification of an Aqueous Ammonium Chloride Solution in a Rectangular Cavity-I. Experimental Study," Int. J. Heat Mass Transfer, Vol. 32, pp. 47-68.

Christenson, M. S., Bennon, W. D. and Incropera, F. P., 1989b, "Solidification of an Aqueous Ammonium Chlc ide Solution in a Rectangular Cavity-II. Comparison of Predicted and Measured Results," Int. J. Heat Mass Transfer, Vol. 32, pp. 69-79.

Christenson, M. S. and Incropera, F. P., 1989c, "Experiments on Solidification of an Aqueous Sodium Carbonate Solution in a Horizontal Cylindrical Annulus," ASME Journal of Heat Transfer, Vol. 111, pp. 998-1005. 
Copley, S. M., Giamei, A. F., Johnson, S. M., and Hornbecker, M. F., 1970, "The Origin of Freckles in Unidirectionally Solidified Castings," Metall. Trans., Vol. 1, pp. 2193-2204.

Crow, S. C. and Champagne, F. H., 1971, "Orderly Structure in Jet Turbulence," J. Fluid Mech., Vol. 48, part 3, pp. 547-591.

Farhang, K., Midha, A., and Bajaj, A., 1987, "A Higher-Order Analysis of Basic Linkages for Harmonic Motion Generation," J. of Mech, Trans, and Automation in Design, Tra .3 ASME, Vol. 109, pp. 301-307.

Felicelli, S. D., Heinrich, J. C., and Poirier, D. R., 1991, "Simulation of Freckles during Vertical Solidification of Binary Alloys," Metall. Trans. B, Vol. 22B, pp. 847-859.

Fisher, K. M., 1981, "The Effect of Fluid Flow on the Solidifications of Industrial Castings and Ingots," PhysicoChem. Hydrodyn., Vol. 2, pp. 311-326.

Flemings, M. C., Mehrabian, R., and Nereo, G. E., 1968a, "Macrosegregation: Part II," Trans. TMS-AIME, Vol. 242, pp. 41-49.

Flemings, M. C. and Nereo, G. E., 1967, "Macrosegregation: Part I," Trans. TMS-AIME, Vol. 239, pp. 1449-1461.

Flemings, M. C. and Nereo, G. E., 1968b, "Macrosegregatiun: Part III," Trans. TMS-AIME, Vol. 243, pp. 50-55.

Fujii, T., Poirer, D. R., and Flemings, M. C., 1979, "Macrosegregation in a Multicomponent Low Alloy Steel," Met. Trans. B, Vol. 10b, pp. 331-339.

Ganeson, S. and Poirer, D. R., 1990, "Conservation of Mass and Momentum for the Flow of InterdendriticLiquid during Solidification," Met. Trans. B, Vol. 21B, pp. 173181.

Gebhart, B., Jaluria, Y., Mahajar, R. L., and Sammakia, B., 1988, BuoyancyInduced Flows and Transport, Hemisphere, New York.

Giamei, A. F. and Kear, B. H., 1970, "On the Nature of Freckles in Nickel Base Superalloys," Metall. Trans., Vol. 1, pp. 2185-2192.

Goldstein, R. J., 1983, "Optical Systems for Flow Measurement: Shadowgraph, Schlieren, and Interferometric Techniques," R. J. Goldstein, Ed., pp. 377-419, Fluid Mechanics Measurements, Hemisphere, New York.

Hopkins, J. A., McCay, M. H., Smith, L. M., and McCay, T. D., 1990, "Optical Methods for Study of Mass Transfer During Metal Model Directional Solidification," Proceedings International Symposium on Manufacturing and Material Processing, Dubrovnik, Yugoslavia.

Huppert, H. E. and Sparks, R. S. J., 1984 "Double-Diffusive Convection due to Crystallization in Magmas," Ann. Rev. Earth Planet Sci., Vol. 12, pp 11-37. 
Huppert, H., 1990, "The Fluid Mechanics of Solidification," J. Fluid Mech., Vol. 212, pp. 209-240.

Jackson, K. A., Hunt, J. D., Uhlmann, D. R., Seward, T. P., 1966, "On the Origin of Equiaxed Zones in Castings," Trans. Metall. Soc. AIME, Vol. 236, pp. 149158.

Kaplan, M. H., 1976, Modern Spacecraft Dynamics and Control, John Wiley and Sons, New York.

Kerr, R. C., Woods, A. W., Worster, M. G., and Huppert, H. E., 1990, "Solidification of an Alloy Cooled from Above, Part 1. Equilibrium Growth," J. Fluid Mech., Vol. 216, pp 323-342.

Kou, S., Poirer, D. R., and Flemings, M. C., 1978, "Macrosegregation in Rotated Remelted Ingots," Met. Trans. B, Vol. 9b, pp 711-719.

McCay, T. D. and McCay, M. H., 1988, "Experimental Measurement of Solutal Layers in Unidirectional Solidification," J. Thermophysics, Vol. 2, pp. 197-202.

McCay, T. D., McCay, M. H., Lowry, S. A., and Smith, L. M., 1989, "Convective Instabilities During Directional Solidification," J. Thermophysics, Vol. 3, No. 3, pp. 345-350.

McCay, T. D. and McCay, M. H., 1990, "Convective Instabilities During Directional Solidification of Light Solute Rejecting Materials," Proceedings International Symposium on Manufacturing and Material Processing, Dubrovnik, Yugoslavia.

McDoniald, R. J. and Hunt, J. D., 1969, "Fluid Motion Through the Partially Solid Regions of a Casting and Its Importance in Understanding A Type Segregates," Trans. AIME, Vol. 245, pp. 1969-1993.

McDonald, R. J. and Hunt, J. D., 1970, "Convective Fluid Motion Within the InterdendriticLiquid of a Casting," Met. Trans., Vol. 1, pp. 1787-1788.

McLean, M., 1983, Directionally Solidified Materials for High Temperature Service, The Metals Society, London.

Mehrabian, R., Keane, M., and Flemings, M. C., 1970a, "Interdendritic Fluid Flow and Macrosegregation; Influence of Gravity," Met. Trans., Vol. 1, pp. 1209-1220.

Mehrabian, R., Keane, M., and Flemings, M. C., 1970b, "Experiments on Macrosegregation and Freckle Formation," Met. Trans., Vol. 1, pp. 3238-3240.

Midha, A., Cipra, R. J., and Farhang, K., 1985, "Analysis and Design of Basic Linkages for Harmonic Motion Generation," J. of Mech, Trans, and Automation in Design, Trans ASME, Vol. 107, pp. 499-507.

Murakami, K. and Okamoto, T., 1984, "Formation of Equiaxed Zone in Castings," Metal Science, Vol 18, pp. 103-111. 
Neilson, D. G., 1991, "Unidirectional Solidification of a Binary Model Alloy and the Effects of Induced Fluid Motion," Ph.D. Thesis, Purdue University, West Lafayette, Indiana.

Neilson, D. G. and Incropera, F. P., 1991, "Unidirectional Solidification of a Binary Alloy and the Effects of Induced Fluid Motion," Int. J. Heat Mass Transfer, Vol. 34, pp. 1717-1732.

Neilson, D. G. and Incropera, F. P., 1992a, "Numerical Study of the Effects of Transport Phenomena on Macrosegregation During Unidirectional Solidification of a Binary Substance," Wărme- und Stoffubertragung, Vol. 27, pp. 1-10.

Neilson, D. G. and Incropera, F. P., 1992b, "Three-Dimensional Considerations of Unidirectional Solidification in a Binary Liquid," Numerical Heat Transfer, (In Press).

Neilson, D. G. and Incropera, F. P., 1992c, "Effect of Rotation on Fluid Motion and Channel Formation during Undirectional Solidification of a Binary Alloy," Int. J. Heat Mass Transfer, (In Press).

Neilson, D. G. and Incropera, F. P., 1992d, "Experimental Study of Unidirectional Solidification of Aqueous Ammonium Chloride in a Cylindrical Mold with and without Rotation," Exp. Heat Transfer, (In Review).

Nishimura, T., Fujiwara, M., and Migashita, H., 1992, "Visualization of Temperature Fields and Double-Diffusive Convection Using Liquid Crystals in an Aqueous Solution Crystallizing along a Vertical Wall," Exp in Fluids, Vol. 12, pp. 245-250.

Norton, T. W., Howell, L. L., and Midha, A., 1992, "On Graphical Synthesis Techniques for Planar Four-Bar Machanisms for Limit Positions and High Performance," Section 4.5, The First Forty Years of Modern Kinematics: A Tribute to Ferdinand Freudenstein, (editor: A. Erdman), John Wiley and Sons, New York.

Okumura, K., Kuwabara, M., Sassa, K., and Muchi, I., 1989, "Theoretical Analysis and Model Experiment on Thermosolutal Convection During Unidirectional Solidification in the Vertical Direction," ISIJ, Vol. 75, pp. 618-625.

Oreper, G. M. and Szekely, J., 1984, "Heat and Fluid Flow Phenomena in Weld Pools," J. Fluid Mech., Vol. 147, pp. 53-79.

Patankar, S. V., 1980, Numerical Heat Transfer and Fluid Flow, Hemisphere Publishing Co., Washington, D. C.

Prescott, P. J., Incropera, F. P., and Bennon, W. D., 1991, "Modeling of Dendritic Solidification Systems: Reassessment of the Continuum Momentum Equation," Int J. Heat Mass Transfer, Vol. 34, pp. 2351-2359.

Prescott, P. J. and Incropera, F. P, 1992a, "Magnetically Damped Convection During Solidification of a Binary Metal Alloy: Numerical Simulations," Proceedings, 29th National Heat Transfer Conference, San Diego, California (In Press). 
Prescott, P. J. and Incropera, F. P, 1992b, "The Effects of Undercooling, Recalescence and Solid Transport on the Solidification of Binary Metal Alloys," Proceedings, 29th National Heat Transfer Conference, San Diego, California (In Press).

Ridder, S. D., Kou, S., and Mehrabian, R., 1981, "Effect of Fluid Flow on Macrosegregatinn in Axi-Symmetric Ingots," Met. Trans. B, Vol. 12B, pp. 435-447.

Sample, A. and Hellawell, A., 1982, "The Effect of Mold Precession on Channel and Macro-Segregation in Ammonium Chloride-Water Analog Castings," Metall.Trans. B, Vol. 13B, pp. 495-501.

Sample, A. K. and Hellawell, A., 1984, "The Mechanisms of Formation and Prevention of Channel Segregation During Alloy Solidification," Metall. Trans. A, Vol. 15A, pp. 2163-2173.

Sarazin, J. R., 1990, "Buoyancy-Driven Convection and Channel Segregation During Solidification in Metallic, Aqueous and Organic Systems," Ph.D. Dissertation, Michigan Technological University, Houghton, Michigan.

Stewart, M. J. and Weinberg, F., 1972, "Fluid Flow Through a Solid-Liquid Dendritic Interface," Met. Trans., Vol. 3, pp. 333-337.

Streat, N. and Weinberg, F., 1974, "Macrosegregation during Solidification Resulting from Density Differences in the Liquid," Met. Trans., Vol. 5, pp. 2539-2548.

Szekely, J. and Jassal, A. S., 1978, “An Experimental and Analytical Study of the Solidification of a Binary Dendritic System," Metall. Trans. B, Vol. 9B, pp. 389-398.

Thompson, M. E. and Szekely, J., 1988, "Mathematical and Physical Modelling of Double-diffusive Convection of Aqueous Solutions Crystallizing at a Vertical Wall," $J$. Fluid Mech., Vol. 187, pp. 409-433.

Turner, J. S., 1974, "Double-Diffusive Phenomena," in Ann. Rev. Fluid Mech., Van Dyke, M., Vincent, W. G., and Wehausen, J. V., Eds., Vol. 6, pp. 37-56.

Viskanta, R., 1985, "Natural Convection in Melting and Solidification," In Natural Convection (Edited by S. Kakac, W. Aung, and R. Viskanta), Hemisphere Publishing Corp., New York.

Voller, V. and Prakash, C., 1987, "A Fixed Grid Numerical Modelling Methodology for Convection-Diffusion Mushy Region Phase-Change Problems," Int. J. Heat Mass Transfer, Vol. 30, pp. 1709-1719.

Wang, L., Laxmanan, V., and Wallace, J. F., 1988, "Gravitational Macrosegregation in Unidirectionally Solidified Lead-Tin Alloys," Met. Trans. A, Vol. 19A, pp. 2687-2694.

Worster, M. G., 1991, "Natural Convection in a Mushy Layer," J. Fluid Mech., Vol. 224, pp. 335-359. 
APPENDICES 


\section{APPENDIX A. THE INDUCEMENT OF CHANNELS USING EXTENDED FINS}

\section{A.1. Introduction}

The present investigation and other similar investigations offer schemes that might effectively eliminate freckle-type segregates in a UDS cast. However, if such freckles could not be eliminated, the next best solution would be to somehow position them in areas of the cast which are not critical to the quality of the final product. Such areas include those which could readily be removed through post-processing or those which are not critical to the structural performance of the cast. Forfeiting the goal of eliminating freckles, a new goal of inducing freckles at predetermined locations in the cast becomes a relatively simple task to achieve.

Observation made during the static UDS of aqueous ammonium chloride indicated that elevated sites which developed along the liquidus interface early in the solidification process tended to become channel locations later in the process. These sites strengthened associated salt fingers and, in turn, the strengthened salt fingers eventually promoted the formation of mature channels. In addition, by efficiently releasing the local interdendritic potential contained deep within the mushy region, the mature channels suppressed development of new channels in the surrounding crystalline structure. Moreover, it is well known that channels formed during the solidification process eventually become freckles in the final cast. Therefore, if hillocks could be produced along the liquidus interface in the infant stages of solidification, the resulting strengthened salt fingers would induce channels, thereby controlling the ultimate distribution of freckles in the cast. 
Short, circular (pin) fins extending from the bottom heat exchanger in a UDS arrangement were chosen to grow hillocks along the liquidus interface and promote channel formation. For use in test section I, six extended fins were employed. Made from 16 gage copper wire, the $31.8 \mathrm{~mm}$ long fins were mounted on a flat, $1.59 \mathrm{~mm}$ thick, stainless steel sheet which was cut to match the dimensions of the heat exchanger surface $(101.6 \mathrm{~mm}$ by $25.4 \mathrm{~mm}$ ). This sheet was then affixed to the heat exchanger. The six fins were placed in a row along the centerline of the heat exchanger, $12.7 \mathrm{~mm}$ from the front and back viewing plates $(z=12.7 \mathrm{~mm})$, and at $x=19.1,31.8,44.5,57.2,69.9$, and $82.6 \mathrm{~mm}$.

The fins worked on a simple principle. Chilled by the heat exchanger, they reduced the temperature of adjoining liquid, thereby producing cold spots along the growth front and promoting dendrites to nucleate in the form of hills. In addition, the fins themselves would act as nucleation sites above the liquidus interface, augmenting the formation of hillocks. Once grown, the hillocks would promote channels at the fin locations and suppress channels in the remaining areas of the cast. The fins only needed to be long enough to start strong channels since the channels tended to be self-perpetuating, propagating with the advancing mushy region.

\section{A.2. Results}

As was observed with previous experiments, crystals began nucleating on the heat exchanger surface within 15 second of applying coolant to the cold plate. Growing rapidly, these early dendrites rejected water-rich fluid which immediately promoted the formation of salt fingers. The extended fins remained free of crystals until approximately two minutes, when dendrites quickly formed on the extended surfaces. These dendrites also rejected water-rich fluid which, after escaping the crystalline structure, was advected upward by buoyancy. 
The dendrites grew radically outward from the circular fins, and roughly following the liquidus temperature isotherms produced by the fins, the liquidus interface rose to engulf the fins thus producing six crystalline pyramids. At seven minutes into the experiment, the pyramids were relatively tall and steep, and the water-rich liquid rejected within each crystal structure coalesced and exited the pyramids at the top of each structure (Fig. A.1a). Away from the hillocks, the planar growth front continued to advance from the bottom wall and salt fingers were still present. However, these salt fingers appeared to be considerably less vigorous than those fingers found in the UDS case without fins. As evidenced by the relative strength of the plumes rising from hillocks, the large interdendritic potential contained in the mushy region was efficiently released by the hillocks, thereby weakening those salt fingers located away from the fins. The dark structures seen at the top of the cavity are air bubbles and do not affect the morphology of solidification.

At 15 minutes into the experiment, the mushy region had advanced upward, decreasing the slopes of the hillocks (Fig. A.1b). However, strong plumes continued to rise from each hillock, and holes or channels had formed in the upper regions of each hillock. As many as five holes developed in the hillocks, but each hole rejected water-rich fluid which coalesced and rose as an ascending column of fluid above the hills. A strong downwash of fluid was observed between the rising plumes as fluid from the melt was entrained downward into the mushy region to feed the robust plumes. In general, the hillocks greatly increased the convective exchange of fluid between the mushy region and the melt. So great was the fluid exchange and subsequent stratification, that doublediffusive interfaces began to form in the upper right section of the melt at 15 minutes (Fig. A.1b).

By 30 minutes, double-diffusive cells were well developed and extended across the entire melt (Fig. A.1c). Vigorous plumes continued to rise from channels in each of the 


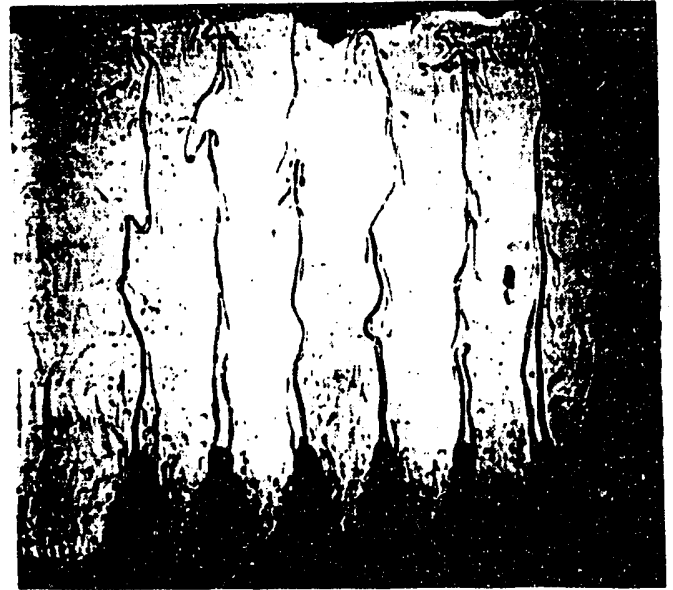

(a)

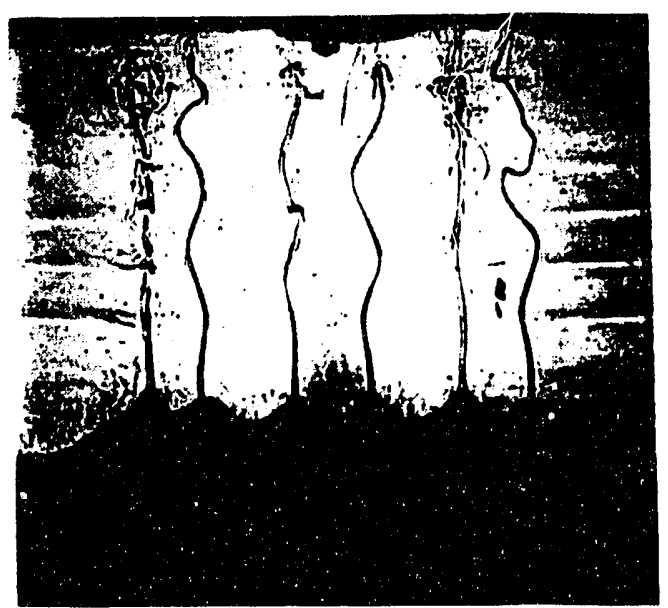

(c)

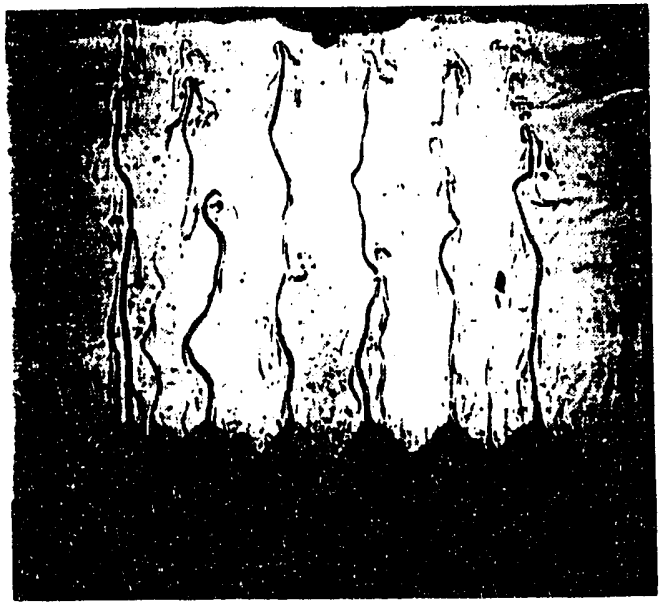

(b)

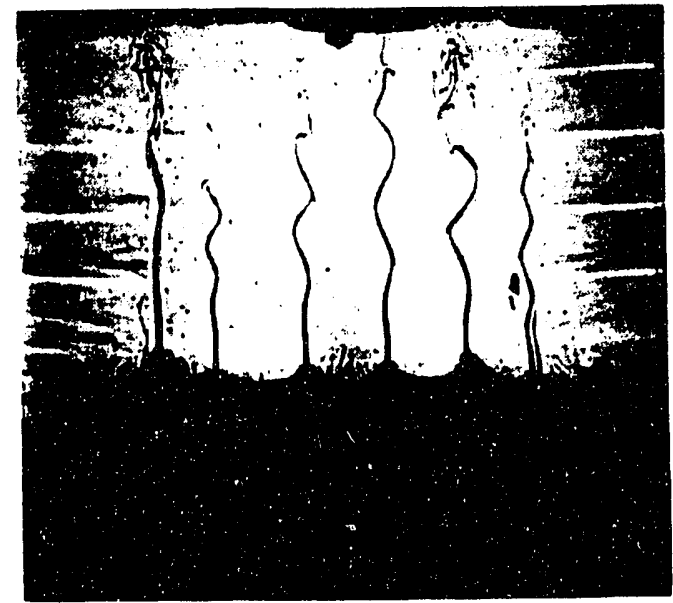

(d)

Figure A.1 Shadowgraph images of UDS with six circular copper fins extending from the heat exchanger at (a) 7, (b) 15, (c) 30, and (d) $60 \mathrm{~min}$. 
hills, while away from the hill, some small, relatively weak salt fingers rose above the liquidus interface.

Finally, at one hour into the experiment, strong plumes continued to rise from the relatively short hillocks and well established double-diffusive convection cells extended throughout the melt (Fig. A.1d). However, an assessment as to how well the fins placed channels at the predetermined sites and of the condition of the crystalline structure away from the fins was necessary.

Figure A.2 shows a direct comparison of freckle location between UDS with and without fins. Whereas channel positions were random without the fins, their deployment effectively confined the channels to the centerline of the cast. More importantly, away from the fin locations, the crystal structure was columnar and free of channels. However, some problems did arise from this scheme.

Around the fins, the crystal growth was not columnar, which is the primary goal of UDS technology. Instead, the crystals grew radially outward from the fins. Also, the robust plumes which were produced by the hillocks quickly stratified the melt which would, in theory, lead to more severe banded segregation in the final cast.

Overall, the fins were successful in positioning freckles at predetermined locations. In fact, similar results could have probably been realized with fewer and shorter fins, as the six $31.8 \mathrm{~mm}$ fins employed in the present investigation seemed to be overly sufficient in releasing interdendritic potential. However, the minimum number of fins needed to release the interdendritic potential is not clear. 


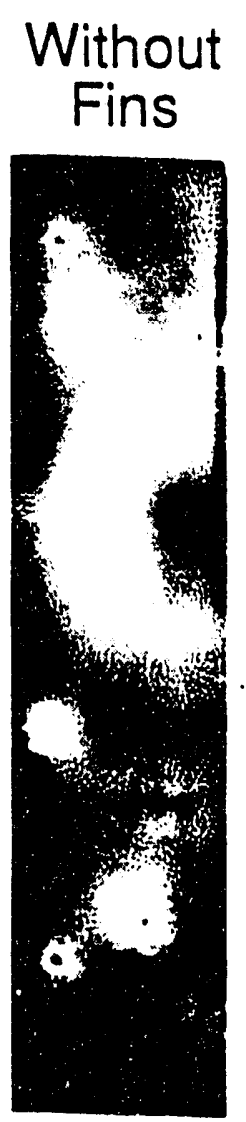

(a)

\section{With}

Fins

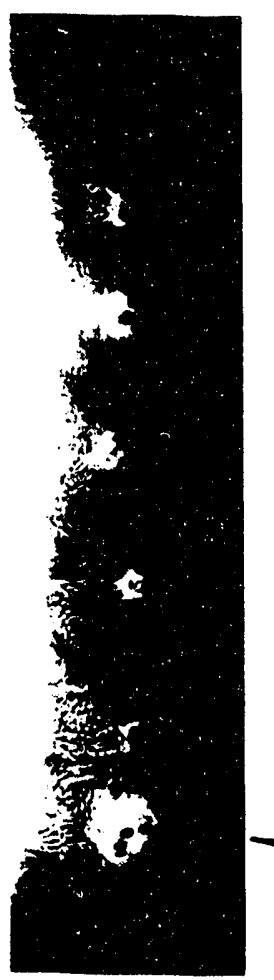

(b)

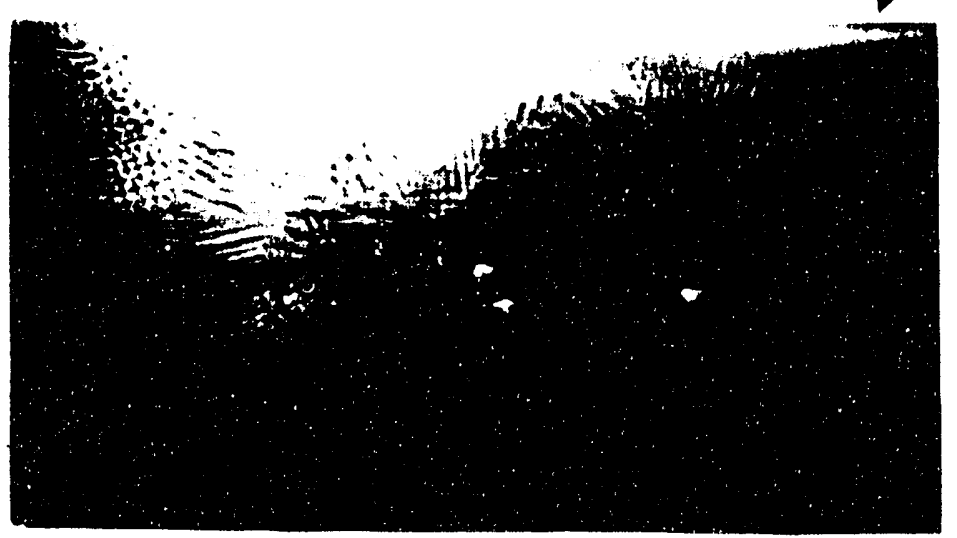

(c)

Figure A.2 Top view showing comparison of grain morphology of UDS with and without fins as viewed from the top. 


\section{APPENDIX B. FLUID CONDITIONS INDUCED BY SINUSOIDAL ROCKING}

In order to understand heat transfer within a rocking box, it is necessary to understand flow conditions induced by the rocking motion alone. For this reason, a simple numerical simulation was perfomed, predicting the flow conditions associated with an oscillating square box. The enclosed fluid was isothermal, and the rocking conditions ( $\mathrm{T}=$ $60 \mathrm{~s}, \theta_{\mathrm{o}}=45^{\circ}$ ) were the same as those applied to case NUDS $4 \mathrm{R}$. The simulation was performed for one minute, with a $25 \times 25$ grid weighted near the boundaries

Figure B.1 shows the flow conditions in a box undergoing the oscillatory rocking motion where convection is induced only by inertial forces associated with the rocking. In the present investigation, a box is rocked sinusoidally about the vertical such that its angular velocity is zero at the positions of largest tilt and maximum at the vertical position. Such is the rotational behavior of the enclosed fluid during the rocking motion in a moving reference frame.

The box in Figure B.1 has been impulsively set into a counterclockwise motion from its maximum tilt of $\theta=-45^{\circ}$. As the box began to rock, the moving reference frame observed the box as stationary while seeing the enclosed fluid body accelerate clockwise at a magnitude matching the angular velocity of the box itself. Rocking up to the vertical position $\left(\theta=0^{\circ}\right)$, the enclosed fluid body rotated at a large clockwise angular velocity (Figure B.1a). Because the enclosed fluid is viscous, drag induced boundary layers developed on each of the enclosure walls. It is only through this viscous drag that the rocking box imparts angular momentum onto the fluid body. Rocking to the far left position, the box comes to rest, and the flow shown in Figure B.1b also corresponds to inertial conditions. Interestingly, there is a slow counterclockwise rotation of the fluid. This rotation results from of the angular momentum imposed on the fluid body by the 

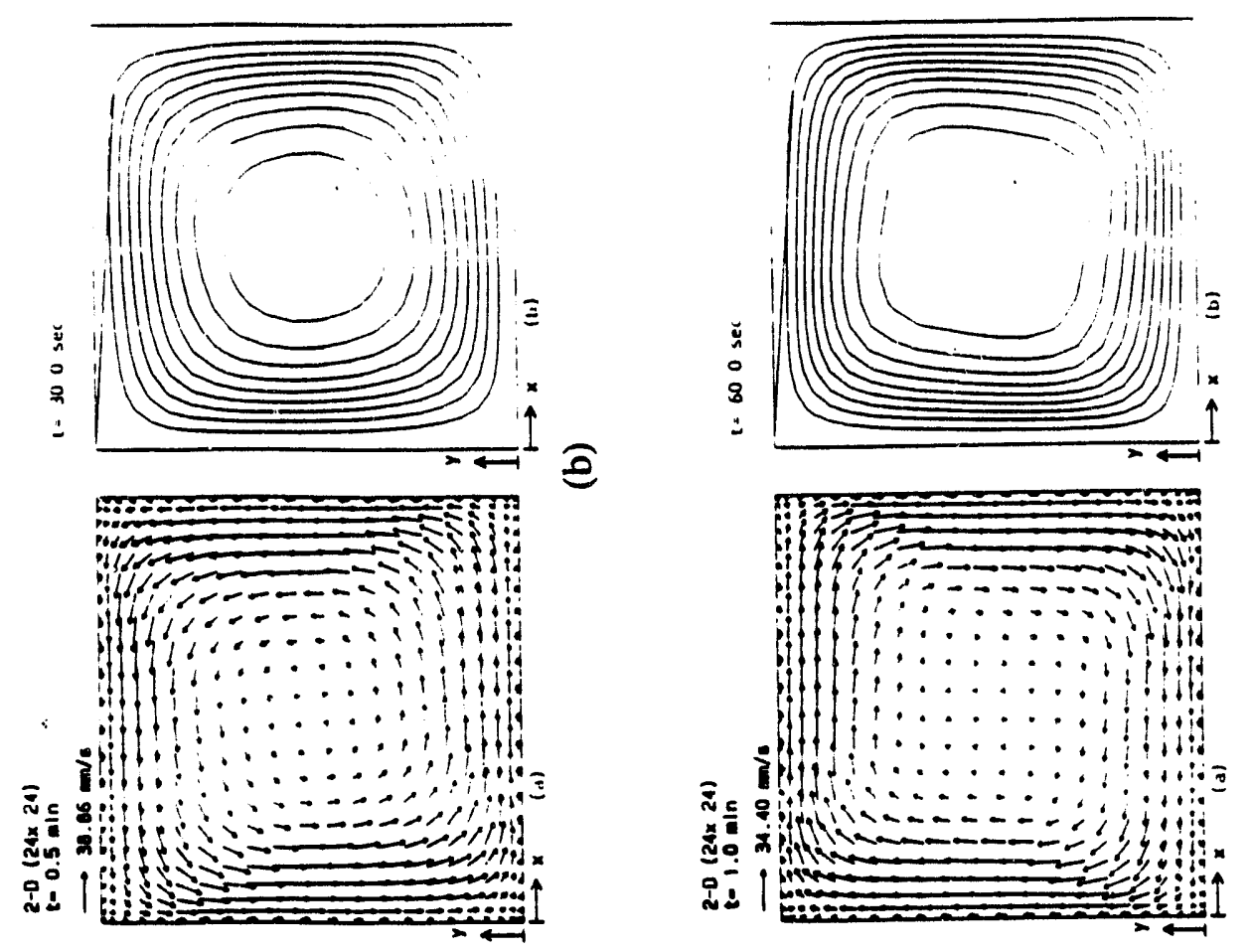

ह
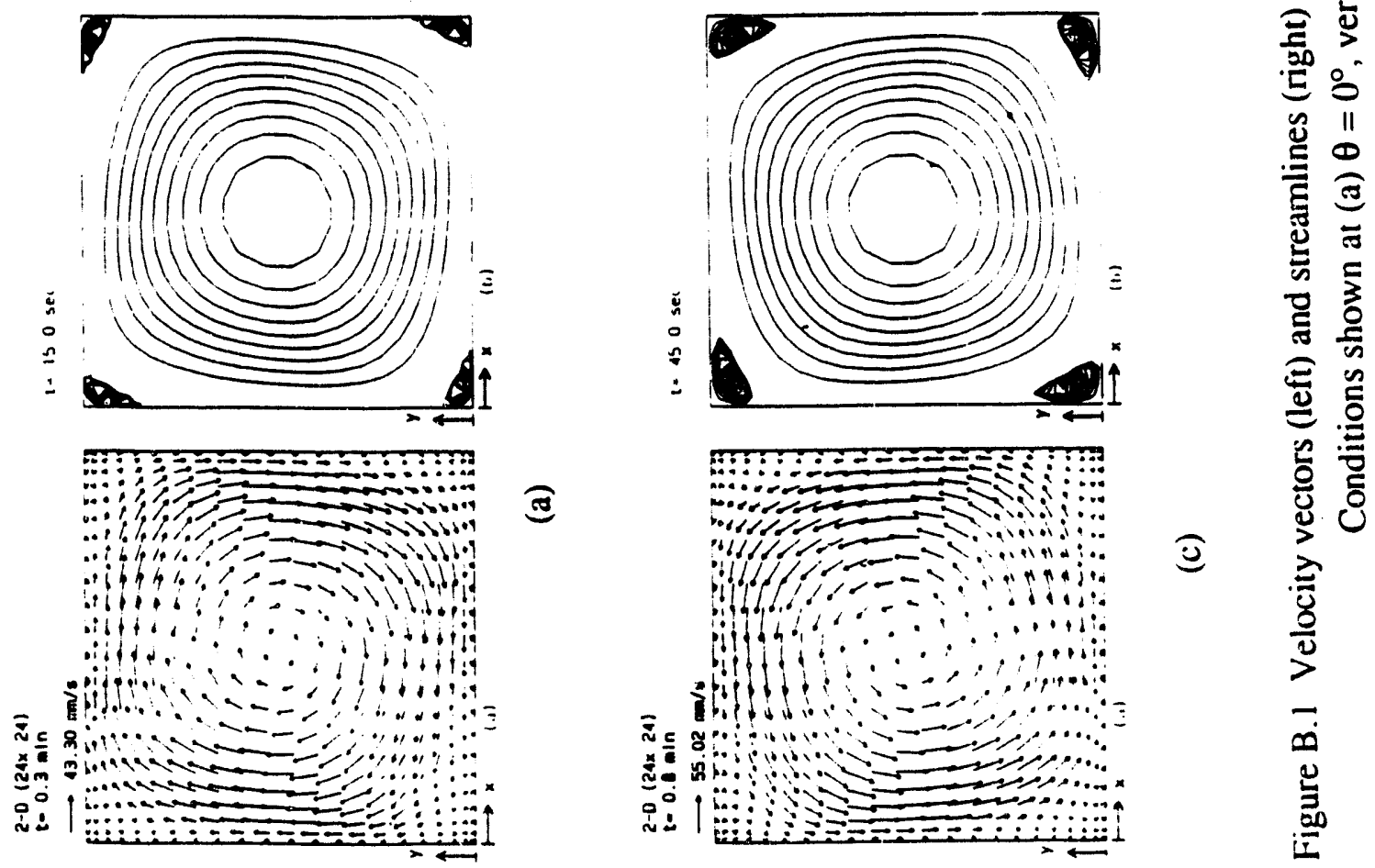
rocking box as it swept from the far right position to the far left position. Rocking back toward the vertical position, the fluid body began to rotate in a counterclockwise direction with respect to the moving reference frame. Reaching the vertical, the fluid body rotation was a maximum as one primary cell rotated counterclockwise within the enclosure (Figure B. 1c), and once again, boundary layers were visible on the enclosure walls. Finally, as the box rotated to the far right thus completing one period, a weak clockwise rotation cell remained in the cavity, and this rotation was again a manifestation of the angular momentum imparted on the fluid body by the rocking walls. 

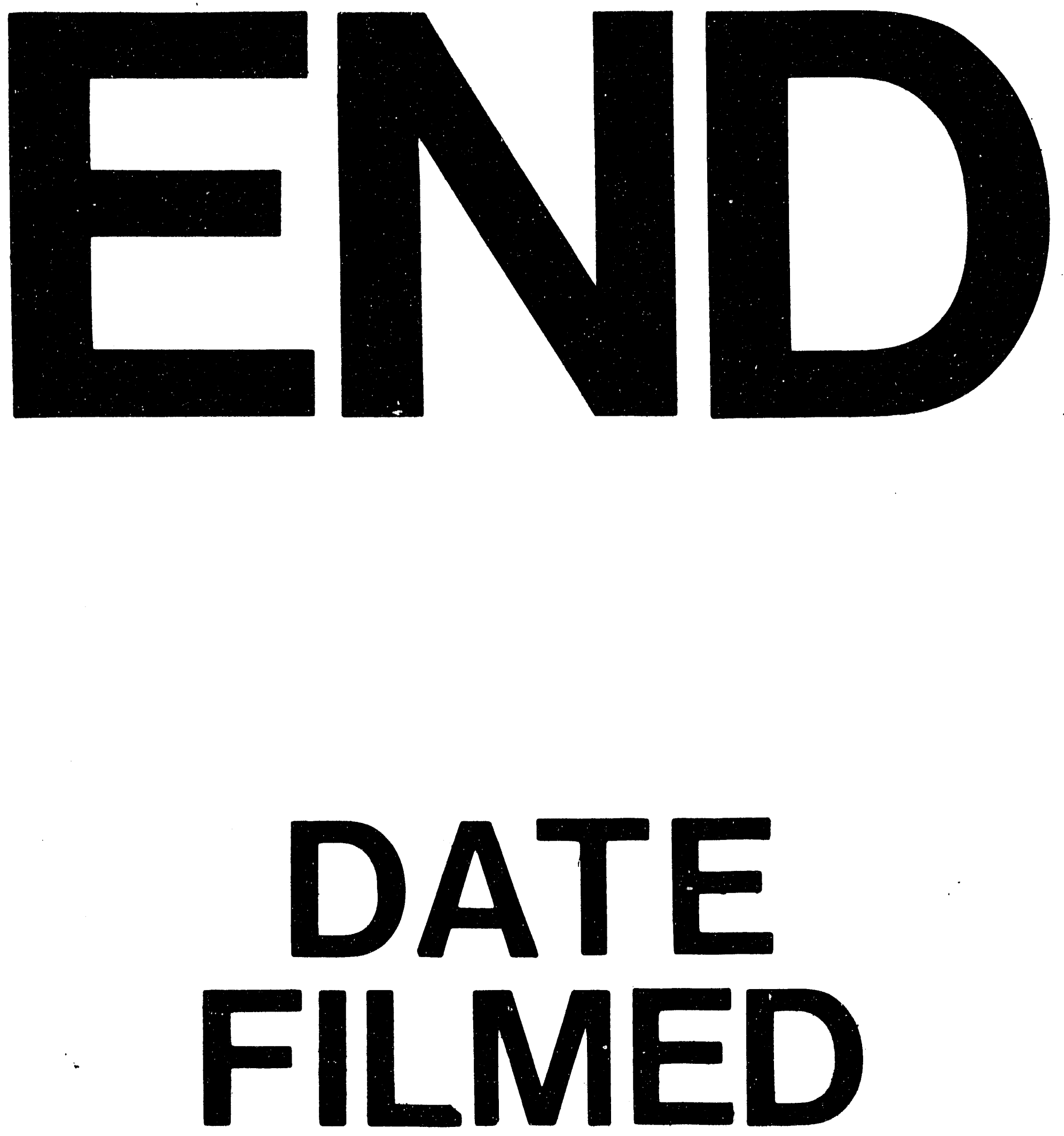

I

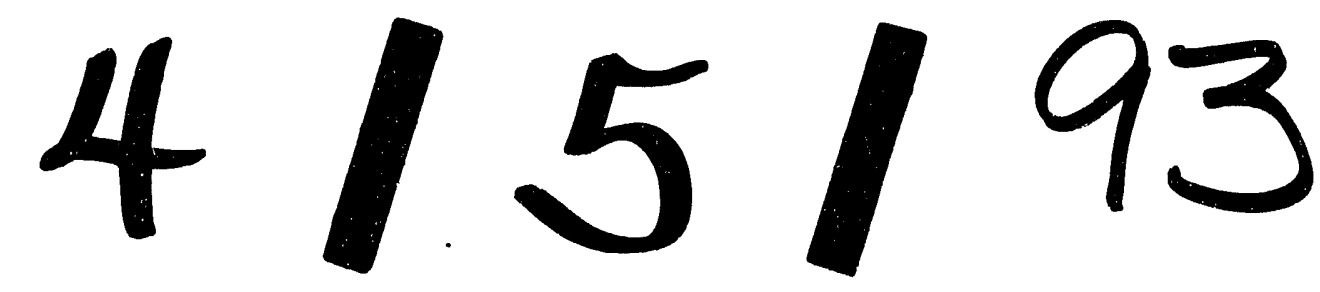


